INSTITUTO DE PESQUISAS ENERGÉTICAS E NUCLEARES

Autarquia associada à Universidade de São Paulo

Obtenção de reatividade subcrítica por meio de medidas de APSD e CPSD utilizando detectores modo pulso no Reator IPEN/MB-01

Seung Min Lee

Tese apresentada como parte dos requisitos para obtenção do Grau de Doutor em Ciências na Área de Tecnologia Nuclear - Reatores

Orientador:

Prof. Dr. Adimir dos Santos

Versão Corrigida

Versão Original disponível no IPEN

São Paulo

2014 


\section{Agradecimentos}

Ao Instituto de Pesquisas Energéticas e Nucleares (IPEN/CNEN) pela infraestrutura e equipamentos necessários à realização deste trabalho.

À Fundação de Amparo à Pesquisa do Estado de São Paulo (FAPESP), pelo financiamento integral, sob o projeto número: 2009/54838-0.

Ao Prof. Dr. Adimir dos Santos, meu orientador, pela competente orientação, muita paciência e amizade, a quem devo meus sinceros agradecimentos.

Ao Dr. Ricardo Diniz, pela indispensável ajuda nos trabalhos experimentais e amizade.

Ao pesquisador Rogério Jerez, pela ajuda na montagem de experimentos e pela operação do reator IPEN/MB-01.

Ao pesquisador Arlindo Gilson Mendoça, pela ajuda na parte da simulação pelo código TORT.

Ao Dr. Pedro Carlos Russo Rossi, pela ajuda na parte da simulação pelo código MCNP5 e MCNP6 e pelo companherismo.

À pesquisadora Graciete Simões de Andrade e Silva, pelo fornecimento de seções de choque.

Ao Dr. Tuffic Madi Filho, pelo apoio na parte de fontes de nêutrons de Am-Be para os experimentos.

Aos membros da banca da defesa, pelas observações e comentários.

Aos colegas do Centro de Engenharia Nuclear, pelo companherismo. 
"No princípio, criou Deus os céus e a terra."

Génesis 1:1 


\title{
Obtenção de Reatividade Subcrítica por meio de Medidas de APSD e CPSD Utilizando Detectores Modo Pulso no Reator IPEN/MB-01
}

\begin{abstract}
RESUMO
Este trabalho apresenta uma nova abordagem experimental para determinar a reatividade de sistemas subcríticos. O método a ser apresentado utiliza o modelo da cinética subcrítica desenvolvido por Gandini e Salvatores e baseia-se apenas em grandezas medidas, tais como a taxa de contagem no detector, e nos parâmetros que surgem do ajuste dos mínimos quadrados APSD (Auto Power Spectral Density) e CPSD (Cross Power Spectral Density), não sendo necessário lidar com as quantidades de maior complexidade como a eficiência de detector. A única hipótese feita neste método foi que a fração efetiva de nêutrons atrasados e o tempo de geração de nêutrons prontos fossem independentes do nível de subcriticalidade do sistema. $\mathrm{O}$ método proposto foi aplicado nas medidas de reatividade de várias configurações subcríticas do reator IPEN/MB-01. Foram realizadas medidas da APSD e CPSD em diversos graus de subcriticalidade (até em torno de $-7000 \mathrm{pcm}$ ). Nos dados das densidades espectrais foram feitos ajustes por meio do método de mínimos quadrados para obter a constante de decaimento pronto $(\alpha)$ e outras grandezas. Com a finalide de melhorar as estatísticas de contagem de nêutrons, fonte externa de nêutrons de Am-Be foi instalada próximo ao núcleo, além da fonte de partida. O método experimental proposto mostra claramente que, a teoria da cinética pontual clássica não descreve a reatividade medida. Em vez disso, a reatividade inferida a partir do modelo da cinética pontual clássica é próxima, em seus valores absolutos, ao índice de subcriticalidade $(\zeta)$ para um determinado arranjo das fontes do experimeno. A concordância dos resultados obtidos por MCNP5 e GPT-TORT, ambos utilizando os dados nucleares da biblioteca ENDF/B-VII.0, com os resultados experimentais correspondentes são de boa qualidade.
\end{abstract}




\title{
Obtainment of the Subcritical Reactivity by mean of Measurement of APSD and CPSD employing pulse mode detectors in the IPEN/MB-01 reactor
}

\begin{abstract}
This work presents a new experimental approach to determine the reactivity levels of subcritical systems. The method employs the subcritical kinetic model developed by Gandini and Salvatores and it is based only on measured quantities such as counting rates of the detectors employed in the experiments and the parameters arising from the least squares fitting of the APSD (Auto Power Spectral Density) and CPSD (Cross Power Spectral Density). Detector efficiencies, quantity required in other procedures such as Neutron Source Multiplication (NSM) method, are not needed in the proposed method. The only hypothesis made in the method was the independence of the effective delayed neutron fraction and the prompt neutron generation time to the subcriticality level of the system. The proposed method was applied to measure the reactivity of several subcritical configurations of the IPEN/MB-01 reactor. Measurements of APSD and CPSD were performed in several degrees of subcriticality (up to around $-7000 \mathrm{pcm}$ ). The spectral densities data were least squares fitted to get the prompt decay mode $(\alpha)$ and other quantities. Beside the startup source of the facility, an external neutron source of Am-Be was installed near the core in order to improve neutron counting statistics. The final experimental results are of good quality. The proposed experimental method shows clearly that the classical point kinetic theory cannot describe the measured reactivity. Instead, the reactivity inferred from this model follows closely the subcriticality index $(\zeta)$ for the source arrangements in the experiment. The agreement of the MCNP5 and GPT-TORT results, both with ENDF/B-VII.0 as the basic nuclear data library, when compared to the corresponding experimental ones was also good.
\end{abstract}




\section{SUMÁRIO}

\section{INTRODUÇÃO}

1.1. Objetivos

1.2. Justificativa

1.3. Revisão Bibliográfica

2. FUNDAMENTAÇÃO TEÓRICA

2.1. Equações da Cinética de Nêutrons

2.2. A Cinética Pontual de Gandini

2.3. Funções de APSD e CPSD

\section{ARRANJOS EXPERIMENTAIS}

3.1. Reator IPEN/MB-01. Configuração do Núcleo

3.2. Arranjos Experimentais e Procedimento de Medidas

3.2.1. Experimento de APSD

3.2.1.1. Procedimento Experimental

3.2.2. Experimento de CPSD

4. PROCESSAMENTO DE SINAIS E AQUISIÇÃO DE DADOS

4.1. APSD

4.2. CPSD

\section{RESULTADOS EXPERIMENTIAS}

5.1. Os resultados da APSD

5.2. Os resultados da CPSD 
6. ANÁLISE TEÓRICA

6.1. Método Estocástico: MCNP

6.1.1. Cálculo da Criticalidade pelo MCNP

6.2. Método Determinístico: GPT-TORT

6.2.1. Método de Ordenadas Discretas

6.2.2. Geração de Seções de Choque

7. RESULTADO DA ANÁLISE TEÓRICA

8. ANÁLISE E DISCUSSÃO DOS RESULTADOS TEÓRICOS E EXPERIMENTAIS

9. CONCLUSÃO

9.1. Trabalhos Futuros

APÊNDICE

REFERÊNCIAS BIBLIOGRÁFICAS 


\section{INTRODUÇÃO}

Nos últimos anos tem havido um grande interesse em sistemas subcríticos (Gandini e Salvatores, 2002; Salvatores, 1996). Esse interesse surgiu parcialmente devido ao surgimento de concepções híbridas denominadas de ADS (Accelerator Driven System) (Rubbia, 2004) e parcialmente devido ao enorme interesse em quantificar experimentalmente a reatividade enquanto o reator se encontra em estado subcrítico.

A reatividade subcrítica pode ser considerada como uma das últimas grandezas não bem descritas no campo da física de reatores. Inúmeras são as situações onde o nível de subcriticalidade é de grande importância. Por exemplo, a criticalização de um PWR em que o reator é ligado e se aproxima da criticalidade é uma das situações em que um bom conhecimento de reatividade subcrítica é exigido. Nessas situações, a reatividade é monitorada pelo método de multiplicação inversa (Shi, Zhu e Tao, 2005), porém, a determinação de reatividade só pode ser realizada por uma maneira relativa, pois não existe um método que determina a reatividade inicial do processo da criticalização.

Existem dois itens importantes relacionados à medida de reatividade, antes e durante o processo da criticalização de reator. O primeiro está ligado com a medida da subcriticalidade durante a aproximação da criticalidade; o segundo, com a estimativa da inserção de barra de controle do reator. A estimação da inserção de barra de controle é realizada convencionalmente através do método de diluição do boro. A medida de subcriticalidade consiste em monitoramento e previsão da condição de multiplicação subcrítica que são essenciais para garantir a segurança na operação de retirada de barras de controle ou no processo de diluição do boro durante a aproximação da criticalidade do reator. Por outro lado, um reatímetro subcrítico digital pode estimar cada grau de inserção das barras de controle. Desta maneira, a 
criticalização do reator poderia ser efetuada com prévia estimação do grau da inserção das barras, e a medida detalhada do grau da inserção poderia ser simplificada ou, até mesmo, eliminada do processo da criticalização.

Atualmente existem vários métodos capazes de estimar o momento da obtenção da criticalidade. Entre eles, o chamado método de multiplicação de fonte de nêutrons (NMS) (Shi e Zhu, 2005) é realizado através da representação gráfica da razão invertida de taxa de contagem de nêutrons, a qual é obtida do detector apropriado para o espectro da fonte, como função da alteração na condição da criticalização do reator, por exemplo, a diluição do boro ou a remoção do banco de controle. Este método assume a distribuição do fluxo de nêutrons no modo fundamental, cuja forma permanece inalterada durante a aproximação da criticalidade. Porém, numa situação real, a distribuição do fluxo de nêutrons é composta do modo fundamental e dos modos harmônicos superiores. Além disso, a forma do fluxo de nêutrons varia significativamente com o nível subcrítico (Shimazu e Naing, 2005; Hoogenboom e Van der Sluijs, 1988).

Outra aplicação importante é na análise de segurança da criticalidade e o subsequente estabelecimento de margens de incertezas e de possível tendência do fator de multiplicação efetivo. Para esta aplicação, o procedimento mais comum é analisar o benchmark apropriado de ICSBEP (Briggs, 2012); i.e., os benchmarks que se assemelham ou que são bem similares à aplicação em consideração. No entanto, as análises dos benchmarks de ICSBEP são confiáveis principalmente em sistemas críticos, e as incertezas e a possível tendência nas situações subcríticas são tomadas da mesma maneira que no sistema crítico; fato que pode incorrer em alguns erros.

A reatividade subcrítica está intimamente relacionada com o modelo de cinética aplicado no sistema. Vários modelos (Gandini, 2004; Dulla, 2006) foram propostos para caracterizar a cinética de reatores subcríticos, principalmente no que tange ao item da reatividade do sistema. Os modelos teóricos sugerem um 
desdobramento da reatividade do sistema em duas componentes; uma, a reatividade do sistema conforme obtida normalmente por meio da teoria da perturbação generalizada (Gandini, 2001), e a outra componente é a reatividade devido à presença da fonte no sistema. Essa última componente é de extrema complexidade para obtenção experimental devido à questão da eficiência do detector que é alterada quando o grau de subcriticalidade do sistema se altera.

Além das dificuldades mencionadas, um grande fator complicador na verificação e validação dos modelos propostos para tratar sistemas subcríticos é a ausência de experimentos adequados que forneçam grandezas físicas que possam ser descritas e tratadas por esses modelos. Nesse aspecto, o reator IPEN/MB-01 pode ser de extrema importância por se tratar de uma instalação com características geométricas e materiais muito bem estabelecidas. O reator IPEN/MB-01 tem sido considerado benchmark internacional em vários experimentos de configurações critica (Dos Santos, et al., 2004a; Dos Santos, et al., 2008) bem como em vários outros experimentos clássicos de Física de reatores (Dos Santos, et al., 2009). Dessa forma, o reator IPEN/MB-01 pode ser considerado de extrema importância em estabelecer parâmetros experimentais para a validação de modelos de cinéticas de sistemas subcríticos.

O objetivo principal do presente trabalho é apresentar um novo método de medida da reatividade subcrítica de sistemas multiplicativos com base no modelo da cinética subcrítica desenvolvido por Gandini e Salvatores (Gandini e Salvatores, 2002; Gandini, 2004). As equações da cinética desse modelo são utilizadas para descrever APSD e CPSD, a partir dos quais são obtidos os parâmetros subcríticos. O método proposto é baseado somente em quantidades medidas, não sendo necessário lidar com as quantidades de maior complexidade como a eficiência de detector. 


\subsection{Objetivos}

Os objetivos do trabalho a ser desenvolvido podem ser enumerados como:

1) Obtenção experimental das APSDs (Auto Power Spectral Density) e CPSDs (Cross Power Spectral Density) em configurações subcríticas do reator IPEN/MB01 utilizando detectores de modo pulso.

2) Obtenção da reatividade subcrítica utilizando o modelo de cinética pontual de Gandini (Gandini, 2004) num ajuste em mínimos quadrados.

3) Obtenção teórica da reatividade subcrítica com o MCNP-5 (MCNP-5 X-5 Monte Carlo Team, 2003). Utilizando as bibliotecas de dados nucleares ENDF/B-VII.0 (Oblozinsky e Herman, 2006), JENDL3.3 (Shibata, 2002), e JEFF3.1 (NEA Data Bank, 2006) e analise da comparação teoria e experimento.

4) Utilizar o sistema acoplado NJOY/AMPX-II/TORT (Dos Santos, et al., 2000) desenvolvido pelo IPEN para análise teórica. O índice de subcriticalidade $(\zeta)$ e a reatividade generalizada $\left(\rho_{\text {gen }}\right)$, assim como outras grandezas de importância para cinética de reatores são determinados com fluxos diretos e fluxos adjuntos calculados pelo TORT (Rhoades, 1991).

\subsection{Justificativas}

A seguir estão as justificativas para a execução deste projeto: 
1) Implementação da técnica experimental de análise de ruído do reator via construção de densidades espectrais com detectores modo pulso (APSD e CPSD). Assim, o grupo de Física de Reatores do Reator IPEN/MB-01 possuirá o domínio de todas as técnicas experimentais de análise de ruído do reator existentes atualmente, a saber: Método Rossi- $\alpha$ (Kuramoto e Dos Santos, 2003; 2006; Uhrig, 1970), Método Feynman- $\alpha$ (Kuramoto e Dos Santos, 2003; 2006; Uhrig, 1970), densidades espectrais adquiridas com detectores em modo corrente (Uhrig, 1970; Diniz e Dos Santos, et al., 2006; Dos Santos, et al., 2006a; Diniz, 2005) e densidades espectrais adquiridas com detectores em modo pulso (Kitamura, 1999). Com relação a esta última, na literatura há registro de apenas um experimento realizado até o presente momento (Diniz, 2005). Atualmente estas técnicas vêm sendo amplamente utilizadas em medidas de reatividades subcríticas em $\operatorname{ADS}$ (Accelerator Driven Systems) (Baeten e Abderrahim, 2003; Pratibha, 2006). A determinação experimental da CPSD constitui fato inédito. Para a APSD existem trabalhos similares (Kitamura, 1999), mas com precisões insatisfatórias para a obtenção de parâmetros integrais e diferenciais de interesse para a área de Física de Reatores. Utilização de um Reator de Pesquisas IPEN/MB-01, integralmente concebido e construído no Brasil no desenvolvimento dessas técnicas experimentais.

2) A utilização de detectores modo pulso, devido à grande variedade disponível no Reator IPEN/MB-01, proporcionará uma grande versatilidade na montagem dos experimentos, possibilitando assim, a realização de medidas no interior do meio multiplicativo e no refletor do reator. Desta forma, é possível estudar efeitos espaciais nas medidas de densidades espectrais. Além disso, a eliminação de eletrômetros e filtros/amplificadores necessários quando utilizados detectores modo corrente, propicia um sinal de melhor qualidade evitando atenuações em frequências acima de $200 \mathrm{~Hz}$, aproximadamente. 
3) Determinação da reatividade subcrítica em varias configurações do reator IPEN/MB-01. A determinação da reatividade subcrítica em reatores nucleares é um procedimento que ainda encontra-se totalmente em aberto. Não existe até a presente data procedimento e nem modelo satisfatório que descreva a cinética de reatores subcríticos.

4) Construção de um sistema de aquisição de dados de ruído do reator baseado em Instrumentos Virtuais capaz de adquirir, processar e analisar em tempo real três experimentos simultaneamente (Rossi- $\alpha$, Feynman- $\alpha$ e densidades espectrais com detectores modo pulso). A utilização de Instrumentos Virtuais possibilita a obtenção de dados com maior precisão, já que grande parte do processamento eletrônico via hardwares (frequentemente fontes de ruídos) é eliminado. Além disso, os resultados de experimentos cujos dados são adquiridos e processados via instrumentação virtual, são obtidos em menor tempo e com menor custo. Este projeto dá continuidade ao processo de modernização do sistema de aquisição de dados do Reator IPEN/MB-01, o qual teve início em 1997.

\subsection{Revisão Bibliográfica}

As primeiras técnicas de medidas de parâmetros subcríticos foram desenvolvidas na década de 1950; e são classificadas como medidas out-of-pile. Basicamente, estas técnicas são baseadas numa variação do grau de subcriticalidade, e medidas da resposta de um detector, a estas mudanças, ou na introdução de um pulso na fonte externa e medidas da resposta do detector a este transiente. Recentemente, com o interesse nos ADS (Maiorino, 2003 e 2005), várias destas técnicas têm sido aplicadas nas instalações de potência zero. Dentre os principais motivos de interesse por este tipo de sistema pode-se citar a sua capacidade de transmutação de resíduos de alta (HLW). Por esta razão, o interesse por um método confiável de determinação de 
subcriticalidade vem crescendo.

O método das Áreas ou de Sjörstrand (Sjörstrand, 1956) sugere determinar a reatividade subcrítica num decaimento de fluxo de nêutrons prontos que segue pulso de nêutrons pela razão de duas áreas, uma de nêutrons prontos e outra de atrasados, no decaimento, considerando um intervalo de alguns milissegundos.

Simmons introduziu o método de Ajuste de Inclinação ou de "Slope Fit" (Simmons e King, 1958) que consiste em determinar a constante de decaimento de nêutrons prontos nos experimentos de fonte pulsada num sistema subcrítico, pela a equação da cinética pontual sem considerar os nêutrons atrasados.

Foi demonstrado por Keepin (Keepin, 1965) que uma retirada súbita ou desligamento de fonte externa de nêutrons de um sistema subcrítico inicialmente em estado estacionário, o fluxo de nêutrons sofre uma queda de nível e a reatividade negativa pode ser determinada pela diferença relativa entre esses níveis de fluxo. Esta técnica foi denominada de método de Source Jerk.

Em tese de doutoramento no IPEN, Kuramoto (Kuramoto, 2007) introduziu um novo método de medida absoluta destes parâmetros, baseado teoricamente no modelo de duas-regiões (Spriggs, 1997) e experimentalmente nas técnicas de análise de ruídos microscópicos, Rossi- $\alpha$ e Feynman- $\alpha$.

Técnica de análise de ruído macroscópico foi iniciada por Moore (Moore, 1958, 1959) após dez anos, aproximadamente, da publicação do primeiro trabalho sobre ruído microscópico. Esta técnica foi verificada logo, experimentalmente, por Cohn (Cohn, 1959, 1960). A análise de ruído macroscópico no domínio de frequência denomina-se análise de densidades espectrais. 
Novas expressões para APSD e CPSD foram obtidas por Diniz (Diniz, 2005) baseado no modelo de reator refletido desenvolvido por Cohn (Cohn, 1962). O autor conclui que no intervalo de frequência de interesse os resultados obtidos pelos modelos refletido e não refletido são idênticos.

Vários métodos de medida de parâmetros subcríticos foram analisados no espectro de nêutrons rápidos a baixa potência dentro do programa MUSE (Multiplication Source Externe) (Gonzales, 2004; Carta, 2004; Mellier, 2005), na instalação MASURCA, França. Estudos sobre determinação de reatividade no espectro térmico e no espectro acoplado térmico-rápido realizaram-se nos experimentos YALINA, na Belarus (Persson, et al., 2005; Persson, 2007).

Por outro lado, as técnicas de medidas de parâmetros cinéticos são baseadas, em muitos casos, em modelos da cinética pontual. Além disso, a maioria dos métodos de cálculos destes parâmetros é baseada em modelos desenvolvidos para sistemas críticos. E recentemente tem sido formado um consenso de que a definição de parâmetros cinéticos de sistemas subcríticos acionados por fonte, ainda necessita uma investigação mais aprofundada, na medida em que conforme demonstrado teoricamente por esta, estes parâmetros variam com o grau de subcriticalidade, e com a perturbação introduzida para cálculo destes parâmetros. Nesse contexto, Gandini (Gandini, 2001) apresentou uma teoria da perturbação generalizada levando em conta o conceito de função importância e de reatividade generalizada. Neste trabalho foi derivada uma nova cinética pontual e foram definidos novos termos que aparecem a partir da redução da cinética espacial para a pontual, como por exemplo, o índice de subcriticalidade.

Mais tarde, Dulla (Dulla, 2006) introduziu um modelo de cinética para sistemas subcríticos que tem a mesma estrutura do modelo de cinética pontual clássica e que é consistente com o conceito de $K_{S}$. 
Simulações computacionais de reatores usam códigos determinísticos ou de Monte Carlo. Dentre os códigos determinísticos, o mais comum é o método de ordenadas discretas, que por sua vez é baseado no método $S_{N}$ desenvolvido por Carlson e Bell (Carlson e Bell, 1958). Mais tarde, Carlson e Lathrop (Carlson e Lathrop, 1965) fizeram uma revisão do método dando ênfase em princípio físico e técnicas de avaliação. Este último trabalho foi seguido por outros que deram desenvolvimento de formulações de geometria $R Z$ e $R \Theta$. O interesse por um código tridimensional determinístico surgiu em Oak Ridge por volta de 1971. Rhoades e Childs estenderam a metodologia de DOT4 à geometria tridimensional (Rhoades e Childs, 1987), o atual TORT. 


\section{FUNDAMENTAÇÃO TEÓRICA}

\subsection{Equações de Cinética de Nêutrons}

A condição de balanço de nêutrons num sistema subcrítico é representada pela seguinte equação de transporte linear integro-diferencial:

$$
\begin{aligned}
& \frac{1}{V(E)} \frac{\partial \Phi(\vec{r}, E, \hat{\Omega}, t)}{\partial t}=-\hat{\Omega} \cdot \nabla \Phi(\vec{r}, E, \hat{\Omega}, t)-\Sigma_{t}(\vec{r}, E) \Phi(\vec{r}, E, \hat{\Omega}, t)+S(\vec{r}, E, \hat{\Omega}, t) \\
& +\int_{4 \pi} d \Omega^{\prime} \int_{0}^{\infty} d E^{\prime} \Sigma_{s}\left(\vec{r}, E^{\prime}, \hat{\Omega}^{\prime} \rightarrow E, \hat{\Omega}\right) \Phi\left(\vec{r}, E^{\prime}, \hat{\Omega}^{\prime}, t\right)+\frac{\chi(\vec{r}, E)}{4 \pi} \int_{4 \pi} d \Omega^{\prime} \int_{0}^{\infty} d E^{\prime} v\left(E^{\prime}\right) \Sigma_{f}\left(\vec{r}, E^{\prime}\right) \Phi\left(\vec{r}, E^{\prime}, \hat{\Omega}^{\prime}, t\right) ;
\end{aligned}
$$

onde

$\Phi(\vec{r}, E, \hat{\Omega}, t)=n(\vec{r}, E, \hat{\Omega}, t) V(E)$ : fluxo angular de nêutrons;

$\Sigma_{t}(\vec{r}, E)$ : seção de choque macroscópica total;

$\Sigma_{f}\left(\vec{r} ; E^{\prime}\right)$ : seção de choque macroscópica de fissão;

$\Sigma_{s}\left(\vec{r} ; E^{\prime}, \hat{\Omega}^{\prime} \rightarrow E, \hat{\Omega}\right)$ : seção de choque macroscópica de transferência;

$v\left(E^{\prime}\right)$ : número médio de nêutrons liberados por fissão;

$\chi(\vec{r}, E)$ : espectro de energia de nêutrons de fissão; $\mathrm{e}$

$S(\vec{r}, E, \hat{\Omega}, t)$ : fonte externa de nêutrons.

As equações que regem o fluxo neutrônico e a densidade dos precursores $\vec{m}$ das I famílias, usando o formalismo de multigrupo, são dadas por: 


$$
\frac{d}{d t}\left(\widehat{V}^{-1} \vec{\Phi}\right)=\left[\widehat{A}+(1-\beta) \widehat{X}_{p} \cdot \widehat{S}_{f}^{G}\right] \cdot \vec{\Phi}+\widehat{X}_{d} \cdot \widehat{\Lambda} \cdot \vec{m}+\vec{s}_{n}
$$

e

$$
\frac{d \vec{m}}{d t}=\widehat{B} \cdot \widehat{S}_{f}^{I} \cdot \vec{\Phi}-\widehat{\Lambda} \cdot \vec{m}
$$

onde $\widehat{A}$ é matriz de transporte, captura e espalhamento, $\widehat{V}^{-1}$ de velocidade de nêutrons. Os demais operadores matriciais são definidos da seguinte maneira:

$$
\begin{array}{ccc}
\vec{\Phi}=\left|\begin{array}{c}
\Phi_{1} \\
\vdots \\
\Phi_{G}
\end{array}\right|_{(G \times 1)}, & \widehat{S}_{f}^{G}=\left|\begin{array}{ccc}
v \Sigma_{f, 1} & \cdots & v \Sigma_{f, G} \\
\vdots & \vdots & \vdots \\
v \Sigma_{f, 1} & \cdots & v \Sigma_{f, G}
\end{array}\right|_{(G \times G)} \\
\vec{m}=\left|\begin{array}{c}
m_{1} \\
\vdots \\
m_{I}
\end{array}\right|_{(I \times 1)}, & \hat{S}_{f}^{I}=\left|\begin{array}{ccc}
v \Sigma_{f, 1} & \cdots & v \Sigma_{f, G} \\
\vdots & \vdots & \vdots \\
\Sigma_{f, 1} & \cdots & v \Sigma_{f, G}
\end{array}\right|_{(I \times G)}, \\
\widehat{B}=\left|\begin{array}{ccc}
\beta_{1} & 0 & 0 \\
0 & \ddots & 0 \\
0 & 0 & \beta_{I}
\end{array}\right|_{(I \times I)}, & \widehat{\Lambda}=\left|\begin{array}{ccc}
\lambda_{1} & 0 & 0 \\
0 & \ddots & 0 \\
0 & 0 & \lambda_{I}
\end{array}\right|_{(I \times I)}, \\
\hat{X}_{p}=\left|\begin{array}{ccc}
0 \\
\chi_{p, 1} & 0 & 0 \\
0 & \ddots & 0 \\
0 & 0 & \chi_{p, G}
\end{array}\right|_{(G \times G)}, & \hat{X}_{d}=\left|\begin{array}{ccc}
\chi_{d, 1} & 0 & 0 \\
0 & \ddots & 0 \\
0 & 0 & \chi_{d, G}
\end{array}\right|_{(G \times G)}, \\
\hat{V}^{-1}=\left|\begin{array}{ccc}
V_{1}^{-1} & 0 & 0 \\
0 & \ddots & 0 \\
0 & 0 & V_{G}^{-1}
\end{array}\right|_{(G \times G)}, & \hat{X}_{d}^{\prime}=\left|\begin{array}{ccc}
\chi_{d, 1} & \cdots & \chi_{d, 1} \\
\vdots & \vdots & \vdots \\
\chi_{d, G} & \cdots & \chi_{d, G}
\end{array}\right|_{(G \times I)}
\end{array}
$$

Em forma matricial, as Eq. (2.2) e Eq. (2.3) podem ser assim expressas: 


$$
\frac{d}{d t}\left|\begin{array}{c}
V^{-1} \vec{\Phi} \\
\vec{m}
\end{array}\right|=\left|\begin{array}{cc}
\left.\hat{A}+(1-\beta) \hat{X}_{p} \cdot \widehat{S}_{f}^{G}\right] & \hat{X}_{d}^{\prime} \cdot \widehat{\Lambda} \\
\hat{B} \cdot \widehat{S}_{f}^{I} & -\hat{\Lambda}
\end{array}\right| \cdot\left|\begin{array}{c}
\vec{\Phi} \\
\vec{m}
\end{array}\right|+\left|\begin{array}{c}
\vec{S}_{n} \\
0
\end{array}\right|
$$

E no estado estacionário, não perturbado a Eq. (2.4) reduz-se a:

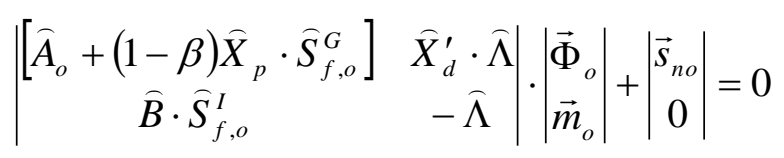

\subsection{A Cinética Pontual de Gandini}

Aqui uma função importância de nêutrons é introduzida; que é relacionada com o nível de potência do sistema subcrítico num estado estacionário. Pois, é mais apropriado usar uma função associada a uma grandeza observável para ponderação de observáveis físico, em vez de usar a função de fluxo adjunto "padrão", $\vec{\Phi}_{0}^{*}$, que é a solução do problema adjunto da Eq. (2.5) sem fonte externa, i.e., $\vec{s}_{n o}=0$. É bom lembrar que a escolha de função importância de nêutrons é arbitraria, e poderia ser, por exemplo, a intensidade da fonte de nêutrons ou a resposta de um dado detector. A função importância de nêutrons escolhida para o modelo da cinética pontual de Gandini, denotada por $\vec{n}_{s, o}^{*}$, é associada à potência normalizada do sistema por:

$$
\widehat{A}_{o}^{*} \vec{n}_{s, o}^{*}+\widehat{S}_{f, o}^{G, T} \cdot\left[(1-\beta) \widehat{X}_{p}+\widehat{B} \cdot \widehat{X}_{d}^{\prime T}\right] \cdot \vec{n}_{s, o}^{*}+\frac{\gamma}{W_{o}} \vec{\Sigma}_{f, o}=0
$$

onde $W_{o}$ é a potência do sistema no estado de referência, $\gamma$ é a energia liberada por fissão. A normalização da potência é dada por:

$$
\frac{\gamma}{W_{0}}\left\langle\vec{\Sigma}_{f, o}, \vec{\Phi}_{0}\right\rangle=1
$$


Além disso, a função importância associada à densidade de precursores é definida da seguinte forma:

$$
\vec{m}_{s, o}^{*} \equiv \hat{X}_{d}^{\prime T} \cdot \vec{n}_{s, o}^{*}
$$

Portanto, as Eqs. (2.6) e (2.7) podem ser expressas em forma matricial por:

$$
\left|\begin{array}{cc}
\left.\hat{A}_{o}^{*}+(1-\beta) \hat{S}_{f, o}^{G, T} \cdot \hat{X}_{p}\right] & \hat{S}_{f, o}^{I, T} \cdot \widehat{B} \\
\hat{\Lambda} \cdot \hat{X}_{d}^{\prime T} & -\hat{\Lambda}
\end{array}\right| \cdot\left|\begin{array}{l}
\vec{n}_{s, o}^{*} \\
\vec{m}_{s, o}^{*}
\end{array}\right|+\left|\begin{array}{c}
\frac{\gamma}{W_{o}} \vec{\Sigma}_{f, o} \\
0
\end{array}\right|=0 .
$$

Agora, assumindo que o transiente foi iniciado num certo instante $t$, como uma perturbação generalizada nos operadores $\hat{A}_{0}, \widehat{S}_{f, o}^{G}, \hat{S}_{f, o}^{I}$ e da fonte $\vec{s}_{n o}$; segue:

$$
\begin{array}{cc}
\widehat{A}_{0} \rightarrow \widehat{A}_{0}+\delta \hat{A}, & \widehat{S}_{f, o}^{G} \rightarrow \widehat{S}_{f, o}^{G}+\delta \widehat{S}_{f}^{G}, \\
\vec{s}_{n o} \rightarrow \vec{s}_{n o}+\delta \vec{s}_{n}, & \widehat{S}_{f, o}^{I} \rightarrow \widehat{S}_{f, o}^{I}+\delta \widehat{S}
\end{array}
$$

que resultam nas perturbações, $\vec{\Phi}_{o} \rightarrow \vec{\Phi}_{o}+\delta \vec{\Phi}$ e $\vec{m}_{o} \rightarrow \vec{m}_{o}+\delta \vec{m}$

Com isso, os fluxos neutrônicos e as densidades dos precursores passam a ser governados pela Eq. (2.4) até o sistema atingir o novo nível estacionário. Ou seja,

$$
\frac{d}{d t}\left|\begin{array}{c}
V^{-1} \vec{\Phi} \\
\vec{m}
\end{array}\right|=\left|\begin{array}{cc}
{\left[\left(\hat{A}_{o}+\delta \hat{A}\right)+(1-\beta) \hat{X}_{p} \cdot\left(\hat{S}_{f, o}^{G}+\delta \widehat{S}_{f}^{G}\right)\right]} & \widehat{X}_{d}^{\prime} \cdot \hat{\Lambda} \\
\hat{B} \cdot\left(\hat{S}_{f, o}^{I}+\delta \widehat{S}_{f}^{I}\right) & -\hat{\Lambda}
\end{array}\right| \cdot\left|\begin{array}{c}
\vec{\Phi} \\
\vec{m}
\end{array}\right|+\left|\begin{array}{c}
\left(\vec{s}_{n o}+\delta \vec{s}_{n}\right) \\
0
\end{array}\right| .
$$

Multiplicando Eq. (2.9) por $\left|\vec{n}_{s, o}^{* T} \quad \vec{m}_{s, o}^{* T}\right|$, , e integrando em todo espaço da fase; chegase as equações em termos da potência do reator, dadas por: 


$$
l_{e f f} \frac{d P_{N}(t)}{d t}=\left(\rho_{g e n}-\alpha_{p d} \beta_{e f f}\right) P_{N}(t)+\alpha_{p d} \sum_{i=1} \lambda_{i} \xi_{i}(t)+\zeta\left(1-P_{N}(t)\right)+\rho_{\text {source }}
$$

e

$$
\frac{d \xi_{i}(t)}{d t}=\beta_{e f f, i} P_{N}(t)-\lambda_{i} \xi_{i}(t)
$$

onde os parâmetros são definidos da seguinte forma:

$$
\begin{aligned}
& P_{N}(t)=\frac{W(t)}{W_{0}(1+q)} ; \\
& \rho_{g e n}=\frac{\left\langle\vec{n}_{s, 0}^{*},\left(\delta \hat{A}+\hat{X} \delta \hat{S}_{f}\right) \vec{\Phi}_{0}\right\rangle}{\left\langle\vec{n}_{s, 0}^{*}, \hat{\bar{X}} \hat{S}_{f, 0} \vec{\Phi}_{0}\right\rangle} ; \\
& q=\frac{\left\langle\delta \hat{S}_{f}, \vec{\Phi}_{0}\right\rangle}{\left\langle\hat{S}_{f, 0}, \vec{\Phi}_{0}\right\rangle} \\
& \rho_{\text {souce }}=\frac{\left\langle\vec{n}_{s, 0}^{*}, \delta \vec{s}\right\rangle}{\left\langle\vec{n}_{s, 0}^{*}, \hat{X} \hat{S}_{f, 0} \vec{\Phi}_{0}\right\rangle} ; \\
& l_{e f f}=\frac{\left\langle\vec{n}_{s, 0}^{*}, \frac{1}{V} \vec{\Phi}_{0}\right\rangle}{\left\langle\vec{n}_{s, 0}^{*}, \hat{\bar{X}}_{f, 0} \hat{S}_{f, 0} \vec{\Phi}_{0}\right\rangle} ; \\
& \zeta=\frac{1}{\left\langle\vec{n}_{s, 0}^{*}, \hat{\bar{X}} \hat{S}_{f, 0} \vec{\Phi}_{0}\right\rangle} \equiv \frac{1-k_{s u b}}{k_{s u b}} \\
& \hat{\bar{X}}=(1-\beta) \hat{X}_{p}+\beta \hat{X}_{d} ; \\
& \xi_{i}=\frac{\left\langle c_{i} \vec{u}, \hat{X}_{d} \vec{n}_{s, 0}^{*}\right\rangle}{\left\langle\vec{n}_{s, 0}^{*}, \hat{\bar{X}} \hat{S}_{f, 0} \vec{\Phi}_{0}\right\rangle}=\frac{\left\langle m_{s, 0}^{*} m_{i}\right\rangle}{\left\langle\vec{n}_{s, 0}^{*}, \hat{\bar{X}} \hat{S}_{f, 0} \vec{\Phi}_{0}\right\rangle} ; \\
& \alpha_{p d}=\frac{\left\langle\vec{n}_{s, 0}^{*}, \hat{X}_{d} \hat{S}_{f, 0} \vec{\Phi}_{0}\right\rangle}{\left\langle\vec{n}_{s, 0}^{*}, \hat{\bar{X}} \hat{S}_{f, 0} \vec{\Phi}_{0}\right\rangle} \\
& m_{s, i, 0}^{*} \equiv m_{s, 0}^{*}=\vec{u}^{T} \hat{X}_{d} \vec{n}_{s, 0}^{*} \text {; } \\
& \beta_{i, e f f}=\frac{\sum_{g=1}^{G} \sum_{j=1}^{J}\left\langle n_{s, 0, g}^{*} c_{j} \chi_{D, g}^{j} \beta_{i, g}^{j} v \sigma_{f, g}^{j} \phi_{g}\right\rangle}{\sum_{g=1}^{G} \sum_{j=1}^{J} \sum_{i=1}^{I}\left\langle n_{s, 0, g}^{*} c_{j} \chi_{D, g}^{j} v \sigma_{f, g}^{j} \phi_{g}\right\rangle} ; \\
& \beta_{\text {eff }}=\sum_{i=1}^{I} \beta_{i, e f f} .
\end{aligned}
$$

onde: 
$l_{\text {eff }}$ : o tempo de geração de nêutrons prontos;

$P_{N}(t)$ : a potência relativa do reator;

$\rho_{\text {gen }}:$ a reatividade generalizada;

$\rho_{\text {source }}$ : a reatividade devido à variação da fonte;

$\alpha_{p d}$ : a relação entre o espectro de nêutrons prontos e o espectro de nêutrons atrasados; $\beta_{\text {eff: }}$ a fração efetiva de nêutrons atrasados;

$\beta_{\text {eff } j \text { j: }}$ a fração de nêutrons atrasados da família $j$;

$\lambda_{j}$ : a constante de decaimento dos precursores de nêutrons atrasados da família $j$;

$\xi_{j}$ : a concentração dos precursores de nêutrons atrasados; e

$\zeta$ : o índice de subcriticalidade.

A semelhança de Eq. (2.10) e Eq. (2.11) às equações usuais da cinética pontual é apenas aparente, e essas equações devem ser usadas com cuidado, uma vez que a potência relativa faz uma grande diferença entre os modelos.

\subsection{Funções de APSD e CPSD}

Através de Eq. (2.10) e Eq. (2.11) e por meio de procedimentos padronizados (Hetrick, 1971; Bell e Glasstone, 1979), a função de transferência do reator de potência zero pode ser prontamente escrita como:

$$
G(\omega)=\frac{1}{\alpha_{p d} \sum_{j=1}^{6} \frac{i \omega \beta_{e f f, j}}{\left(i \omega-\lambda_{j}\right)}-i \omega l_{e f f}-\rho_{g e n}+\zeta}
$$

onde $\omega$ é a frequência angular, e assumiu-se que existem seis grupos de nêutrons atrasados. A dedução da Eq. (2.12) encontra-se no Apêndice A. 
Ao longo da região de frequência em que $\omega \gg \lambda_{i}$, a Eq. (2.12) pode ser simplificada em:

$$
G(\omega)=\frac{1}{\alpha_{p d} \sum_{j=1}^{6} \beta_{e f f, j}-i \omega l_{e f f}-\rho_{g e n}+\zeta}=\frac{1}{\alpha_{p d} \beta_{e f f}-i \omega l_{e f f}-\rho_{g e n}+\zeta}
$$

Agora, considerando uma cadeia eletrônica padrão para um detector de modo pulso, a APSD do ruído de nêutrons é dada por:

$$
\Phi(\omega)=\frac{A}{\omega^{2}+B^{2}}+C
$$

onde $A$ e $C$ são constantes, sendo $C$ o ruído não correlacionado, e

$$
B=\frac{\rho_{g e n}-\alpha_{p d} \beta_{e f f}-\zeta}{l_{e f f}}
$$

é o análogo da constante de decaimento pronto, $\alpha$, para o modelo da cinética pontual clássica.

Os parâmetros $A, B$ e $C$ podem ser obtidos pelo ajuste da APSD experimental através de um procedimento de ajuste de mínimos quadrados.

Além disso, em um estado estacionário Eq. (2.10) e Eq. (2.11) podem ser acopladas resultando em:

$$
\rho_{\text {gen }} P_{N}+\zeta\left(1-P_{N}\right)+\rho_{\text {source }}=0 .
$$

Através das Eqs. (2.15) e (2.16), assumindo que $\rho_{\text {source }}=0$, os parâmetros de 
interesse, $\zeta$ e $\rho_{g e n}$, são dados por:

$$
\zeta=-\left(B l_{e f f}+\alpha_{p d} \beta_{e f f}\right) P_{N}
$$

e

$$
\rho_{g e n}=\left(B l_{e f f}+\alpha_{p d} \beta_{e f f}\right)\left(1-P_{N}\right) .
$$

Nas Eqs. (2.17) e (2.18), os parâmetros $l_{\text {eff }}$ e $\beta_{\text {eff já são conhecidos a partir das }}$ experiências anteriores (Dos Santos, 2009). Os parâmetros $l_{\text {eff }}$ e $\beta_{\text {eff }}$ são considerados independentes do nível de subcriticalidade. Para utilizar as Eqs. (2.17) e (2.18), a potência relativa $P_{N}$ de dois estados consecutivos deve ser obtida. Por analogia com o modelo da cinética pontual, a potência para um determinado estado é dado por (Cohn, 1960; Suzuki, 1966):

$$
P=\frac{2 R^{2}(\gamma D) l_{e f f}^{-2}}{(B)^{2}\left(\Phi^{p}-C\right)},
$$

onde:

$R$ : a corrente convertida em voltagem para detectores de modo corrente, ou contagem para detectores de modo pulso;

$\gamma$ : a energia liberada por fissão em Joule;

$D:$ o fator de Diven;

$B$ : a constante de decaimento de nêutrons prontos $\alpha$ ou sua analogia, dependendo do modelo da cinética utilizada;

$\Phi^{p}$ : valor médio da APSD do primeiro plateau $\mathrm{em} \mathrm{V}^{2} / \mathrm{Hz}$ para detector de modo corrente, ou Counts ${ }^{2} / \mathrm{Hz}$ para detector de modo pulso;

$C$ : valor médio do ruído não correlacionado.

Agora, considerando dois estados $a$ e $b$, dos quais o estado $b$ é mais subcrítico do que o estado $a$, e supondo que $l_{\text {eff }}$ é independente do nível de subcriticalidade, a potência relativa entre estes dois estados pode ser escrita como: 


$$
P_{N}=\frac{P_{b}}{P_{a}}=\frac{R_{b}^{2} B_{a}^{2}\left(\Phi_{a}^{p}-C_{a}\right)}{R_{a}^{2} B_{b}^{2}\left(\Phi_{b}^{p}-C_{b}\right)} .
$$

Assim, através das Eqs. (2.17), (2.18) e (2.20) os parâmetros $\zeta$ e $\rho_{\text {gen }}$ podem ser obtidos de forma puramente experimental. Nas Eqs. (2.17) e (2.18), $\alpha_{p d}$ é considerado como unidade para as propostas deste trabalho. Esta não é uma hipótese, pois o $\beta_{\text {eff }}$ utilizado aqui não é o beta físico como na equação do Gandini (embora denotado por $\beta_{\text {eff }}$ ), mas sim o beta efetivo:

$$
\beta_{i, e f f}=\beta_{i} \cdot \frac{\widehat{X}_{d, i} \cdot \vec{n}_{s, o}^{*}}{\widehat{X} \cdot \vec{n}_{s, o}^{*}}
$$

Portanto,

$$
\alpha_{p d} \cdot \beta=\sum_{i=1}^{I}\left(\frac{\left\langle\vec{n}_{s, 0}^{*}, \vec{\chi}_{d} \vec{S}_{f, 0} \vec{\Phi}_{0}\right\rangle}{\left\langle\vec{n}_{s, 0}^{*}, \bar{\chi}_{f, 0} \vec{\Phi}_{0}\right\rangle} \cdot \frac{\hat{X} \cdot \vec{n}_{s, o}^{*}}{\widehat{X}_{d, i} \cdot \vec{n}_{s, o}^{*}} \cdot \beta_{i, e f f}\right) \cong \beta_{e f f} .
$$

No Apêndice B encontram-se os detalhes da dedução da expressão da $P_{N}$ para o caso da APSD. A potência relativa, no caso da CPSD pode ser deduzida de maneira análoga, e é dada por (Diniz, 2005):

$$
P_{N}=\frac{P_{b}}{P_{a}}=\frac{\left(R_{1, b} R_{2, b}\right) B_{a}^{2} \Phi_{a}^{p}}{\left(R_{1, a} R_{2, a}\right) B_{b}^{2} \Phi_{b}^{p}},
$$

onde os subíndices 1 e 2 se referem ao detector 1 e ao detector 2 , respectivamente, e os $a$ e $b$ são, como no caso da APSD, dos estados $a$ e $b$, sendo $b$ o mais subcrítico. Ou seja, $R_{l, b}$ é, por exemplo, a taxa de contagem medida no detector 1 no estado mais 
subcrítico. O parâmetro $B$ é, novamente, obtido pelo ajuste dos mínimos quadrados da curva dada pela Eq. (2.22) aos pontos experimentais da CPSD, e $\Phi^{p}$, pela média dos valores da CPSD na região do patamar.

$$
\Phi(\omega)=\frac{A}{\omega^{2}+B^{2}}
$$

O parâmetro $A$ é, agora, definido como sendo:

$$
A \equiv \frac{2 v D}{l_{e f f}^{3} N} \cdot R_{1} \cdot R_{2} \cdot q^{2} \cdot\left(H_{e 1}(\omega) H_{f 1}(\omega)\right)\left(H_{e 2}(\omega) H_{f 2}(\omega)\right),
$$

onde:

$H_{e 1}(\omega)$ e $H_{e 2}(\omega)$ são funções transferência de "scaler" dos detectores 1 e 2, respectivamente, em Volt/R, $\mathrm{e}$

$H_{f 1}(\omega)$ e $H_{f 2}(\omega)$ são funções transferência de demais módulos eletrônicos, associadas aos detectores 1 e 2 . 


\section{ARRANJOS EXPERIMENTAIS}

\subsection{Reator IPEN/MB-01. Configuração do Núcleo.}

O reator IPEN/MB-01 utilizado para este experimento é um reator de potência zero, cuja potência máxima está limitada a 100W. Uma descrição detalhada do reator encontra-se nas referências (Dos Santos, et al., 2004a; 2006b). O núcleo do reator padrão consiste em 28 x 26 varetas, das quais 680 são de combustível, que são compostas de pastilhas de $\mathrm{UO}_{2}$ com enriquecimento de $4.3486 \%$ em massa de ${ }^{235} \mathrm{U}$. Tais pastilhas são contidas dentro do encamisamento de aço inoxidável (SS-304). A Fig. 3.1 mostra alguns detalhes do núcleo do reator. Esta configuração do núcleo (28 x 26) está ilustrada na Fig. 3.2. Os símbolos AC e AS referem-se, respectivamente, às barras de controle e de segurança. As barras de controle contêm uma liga de Ag-In-Cd, ao passo que, as barras de segurança são preenchidas por $\mathrm{B}_{4} \mathrm{C}$. Os números de 3 dígitos abaixo desses três símbolos (AC, AS e UC) são de identificação de cada vareta. Cada conjunto de 12 barras, tanto de controle quanto de segurança, formam um banco; ou seja, dois bancos de cada tipo de barra estão posicionados em diagonal. Doravante, os bancos de controle e os bancos de segurança serão denotados, respectivamente, por $\mathrm{BC}$ e BS.

Para este trabalho, as varetas de combustíveis da última fileira em cada face foram removidas, i.e., no total, 104 varetas de combustíveis foram retiradas do núcleo padrão. Esta configuração do núcleo mostrada na Fig. 3.3, é uma das configurações críticas de LEU.COMP.THERM.082 (Dos Santos, et al., 2006b). Desta maneira, praticamente todo o excesso da reatividade do núcleo foi removido ( $K_{\text {eff }}$ medido foi igual a 1.00010, com os bancos de controle e de segurança totalmente retirados). A reatividade foi controlada por um sistema de dois bancos de controle: BC1 e BC2. Os dois bancos de segurança, BS1 e BS2, foram mantidos na posição de remoção total durante toda a operação. Esta posição equivale à de $135 \%$ retirados, pois o material 
absorvedor fica a uma distância equivalente a 35\% do comprimento do núcleo ativo acima do próprio núcleo ativo. Essa configuração do núcleo, de 26 × 24 varetas de combustíveis, foi escolhida por duas razões: primeiro, para poder posicionar os bancos de controle inicialmente numa posição de maior remoção possível; e segundo, para obter um nível mais baixo possível de subcriticalidade com os bancos de controle totalmente inseridos.

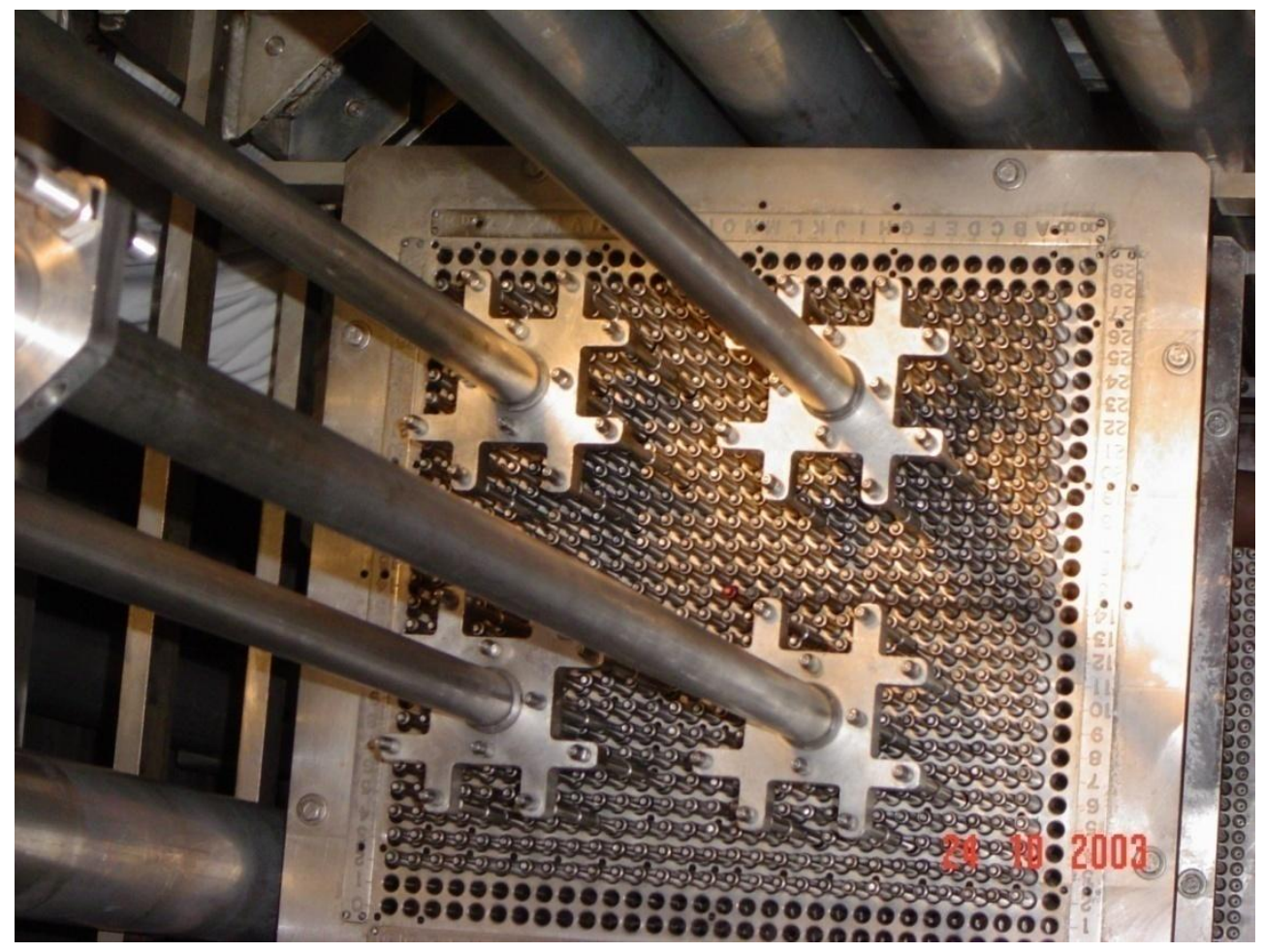

Fig. 3.1. Detalhes do núcleo do reator IPEN/MB-01 


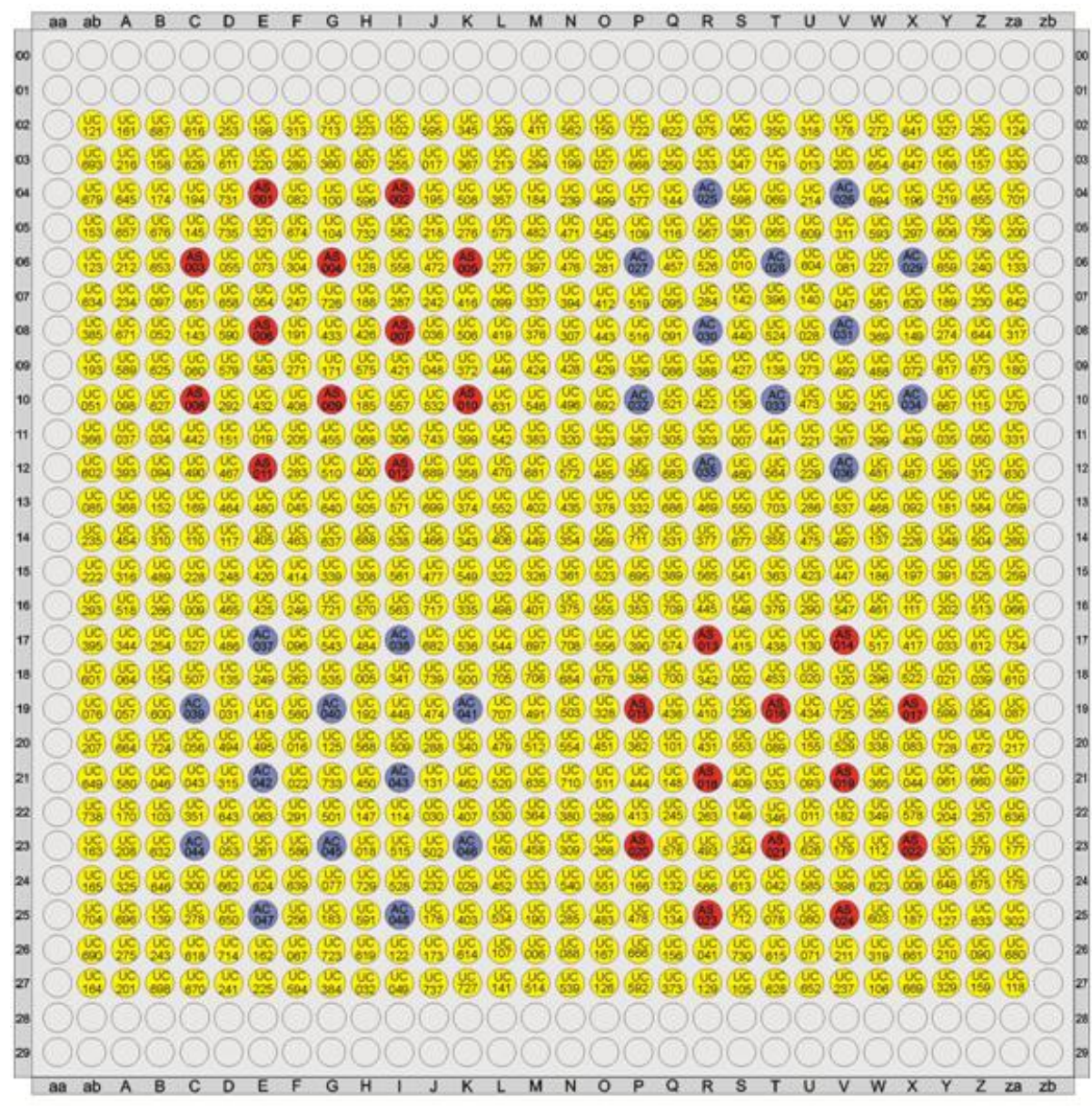

LEGENDA:
(v) Vareta Combustivel
(19) Vareta Absorvedora de Controle
(2) Vareta Absorvedora de Segurança

Fig. 3.2. Configuração padrão do Reator IPEN/MB-01, de 28 x 26; onde as varetas de combustíveis são denotadas por UC, e os símbolos AC e AS representam, respectivamente, as varetas de banco de controle (BC), e as de banco de segurança (BS). 




Fig. 3.3. Configuração experimental, de 26 x 24, utilizada para este experimento, onde as varetas de combustíveis da fileira periférica em cada face da configuração pardrão $(28$ x 26) foram removidas. 


\subsection{Arranjos Experimentais e Procedimento de Medidas}

Há vários aspectos similares entre o experimento da APSD e da CPSD realizados para este trabalho, no que tange ao arranjo e ao procedimento experimental, embora haja um intervalo razoável do tempo entre os dois experimentos. De fato, ambos os experimentos foram realizados com núcleo da configuração 26 x 24; todos os detectores utilizados no experimento da APSD foram empregados no experimento da CPSD; a fonte de Am-Be com intensidade de 1Curie (2.6 $10^{6}$ nêutrons/seg) foi utilizada em ambos os casos; entre outros. Por esta razão, optou-se apresentar, primeiramente, o experimento da APSD com maiores detalhes e, ao tratar do experimento da CPSD, serão destacadas as diferenças e algumas observações.

\subsubsection{Experimento de APSD}

Devido à falta de um detector capaz de abranger toda a gama de reatividade dos experimentos, foi necessário utilizar detectores de sensibilidades diferentes. Três detectores de modo pulso foram utilizados para os experimentos de APSD, os quais são listados a seguir, em ordem crescente da sensibilidade:

a) de $\mathrm{BF}_{3}$, de sensibilidade $15 \mathrm{cps} / \mathrm{nv}$ (Reuter-Stockes);

b) de ${ }^{3} \mathrm{He}$, de sensibilidade 54,3 cps/nv (Centronic); doravante, o detector de ${ }^{3} \mathrm{He}$ de sensibilidade média; e

c) de ${ }^{3} \mathrm{He}$, de sensibilidade $186 \mathrm{cps} / \mathrm{nv}$ (Centronic); doravante, o detector de ${ }^{3} \mathrm{He}$ de sensibilidade alta.

Os três detectores supra citados são a gás proporcional; o de $\mathrm{BF}_{3}$ é baseado na

reação ${ }^{10} \mathrm{~B}(\mathrm{n}, \alpha){ }^{7} \mathrm{Li}$, e dos ${ }^{3} \mathrm{He}$, na reação ${ }^{3} \mathrm{He}(\mathrm{n}, \mathrm{p}){ }^{3} \mathrm{He}$; sendo que, a seção de choque da segunda reação é maior que a da primeira. As seções de choque destas duas reações 
são mostradas na Fig. 3.4. A referência (Knoll, 2000) apresenta o princípio de operação do detector proporcional e, fornece uma descrição mais completa dos contadores de nêutrons mais utilizados. A possibilidade de operar à pressão significativamente superior permite eficiência maior aos detectores de ${ }^{3} \mathrm{He}$; enquanto a pressão do gás de $\mathrm{BF}_{3}$ é normalmente limitada entre 0.5 a 1 atm., pois, o $\mathrm{BF}_{3}$ perde desempenho como gás multiplicativo a pressões superiores.

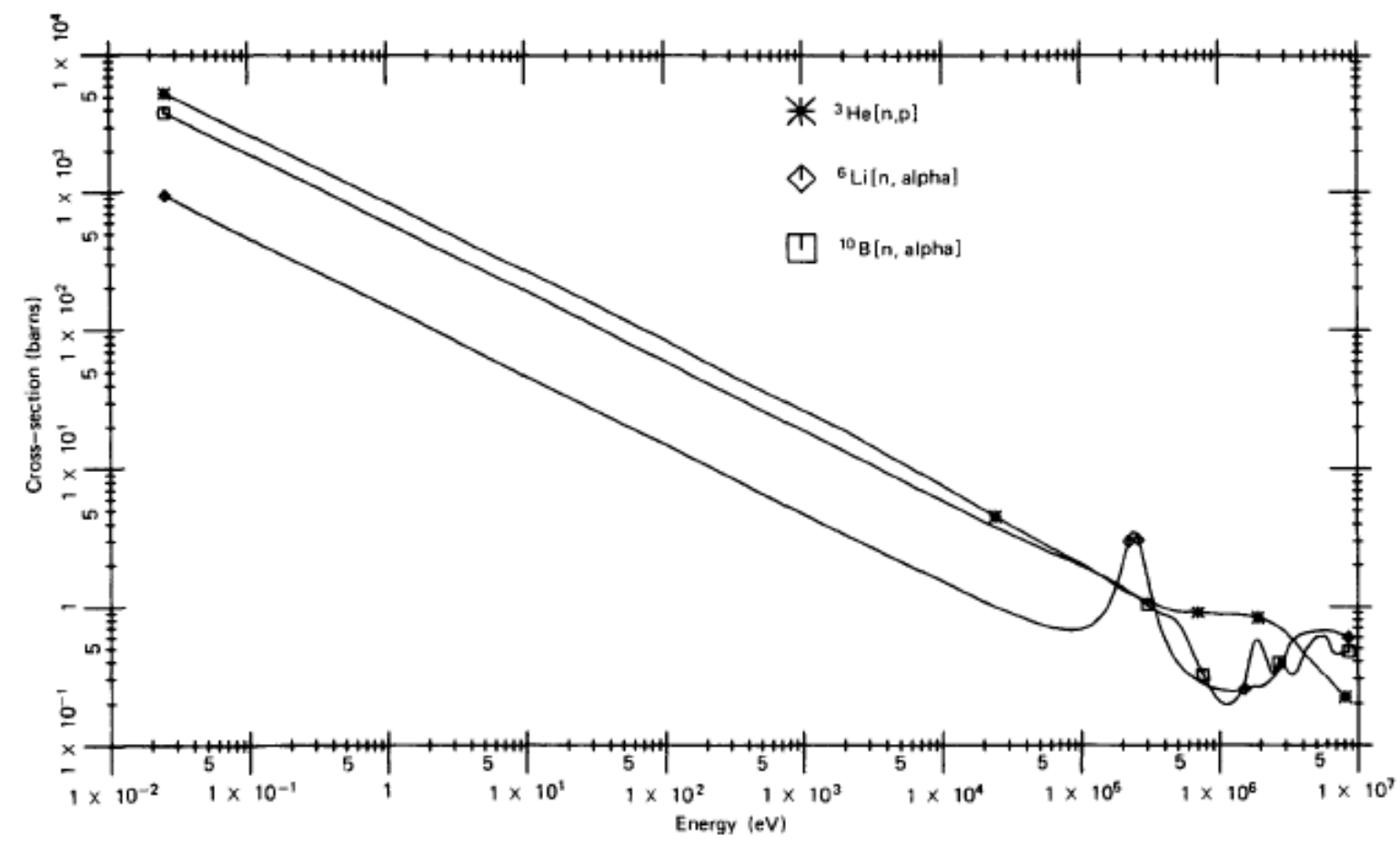

Fig. 3.4. Seção de choque em função de energia de nêutrons para algumas reações de interesse em detecção de nêutrons (Knoll, 2000).

Os detectores experimentais foram posicionados, um de cada vez, no plano y-z, a $12 \mathrm{~cm}$ das varetas de combustíveis da última fileira, como ilustrado na Fig. 3.5. Os eixos axiais dos detectores de forma cilíndrica foram sempre mantidas alinhadas na direção do eixo z. Os detectores experimentais foram colocados dentro de tubos de alumínio, preenchidos com discos de polietileno, conforme mostrado na Fig. 3.6. Vários discos de polietileno têm perfurações circulares no centro para acomodar os 
detectores. O diâmetro interno e a espessura dos discos variam dependendo do tamanho do detector a ser inserido. Ou seja, o arranjo dos discos dentro do tubo é alterado de acordo com o tamanho do detector. A reatividade total inserida por todos os tubos de alumínio foi medida e é dada por $21( \pm 1.2) \mathrm{pcm}$, conforme relatado em (Dos Santos, et al., 2004a)

Para melhorar as estatísticas das contagens dos detectores e, assim, a resolução das densidades espectrais, fontes de nêutrons adicionais de Am-Be de intensidade de $100 \mathrm{~m} C i$ ou $1 C i$ foram colocadas no meio da região ativa, na face leste do núcleo, entre as linhas 14 e 15, encostadas às respectivas varetas de combustíveis, conforme ilustrado na Fig. 3.7.

A fonte de Am-Be é confinada dentro de um cilindro acrílico que, por sua vez, é montada num suporte acrílico elaborado especialmente para este fim, como ilustrado na Fig. 3.8. O cilindro possui pequenos orifícios para permitir entrada e saída da água. Além das fontes adicionais, o reator conta com uma fonte de partida localizada embaixo do núcleo e, também, a fonte intrínseca do combustível. A fonte intrínseca do combustível surge de fissão espontânea do urânio e deve ser modelada em toda a região ocupada por varetas de combustíveis. A reatividade inserida pelo suporte acrílico foi medido e seu valor é de $-10.31( \pm 0.02) p c m$. A incerteza é dada por desvio padrão da média. 


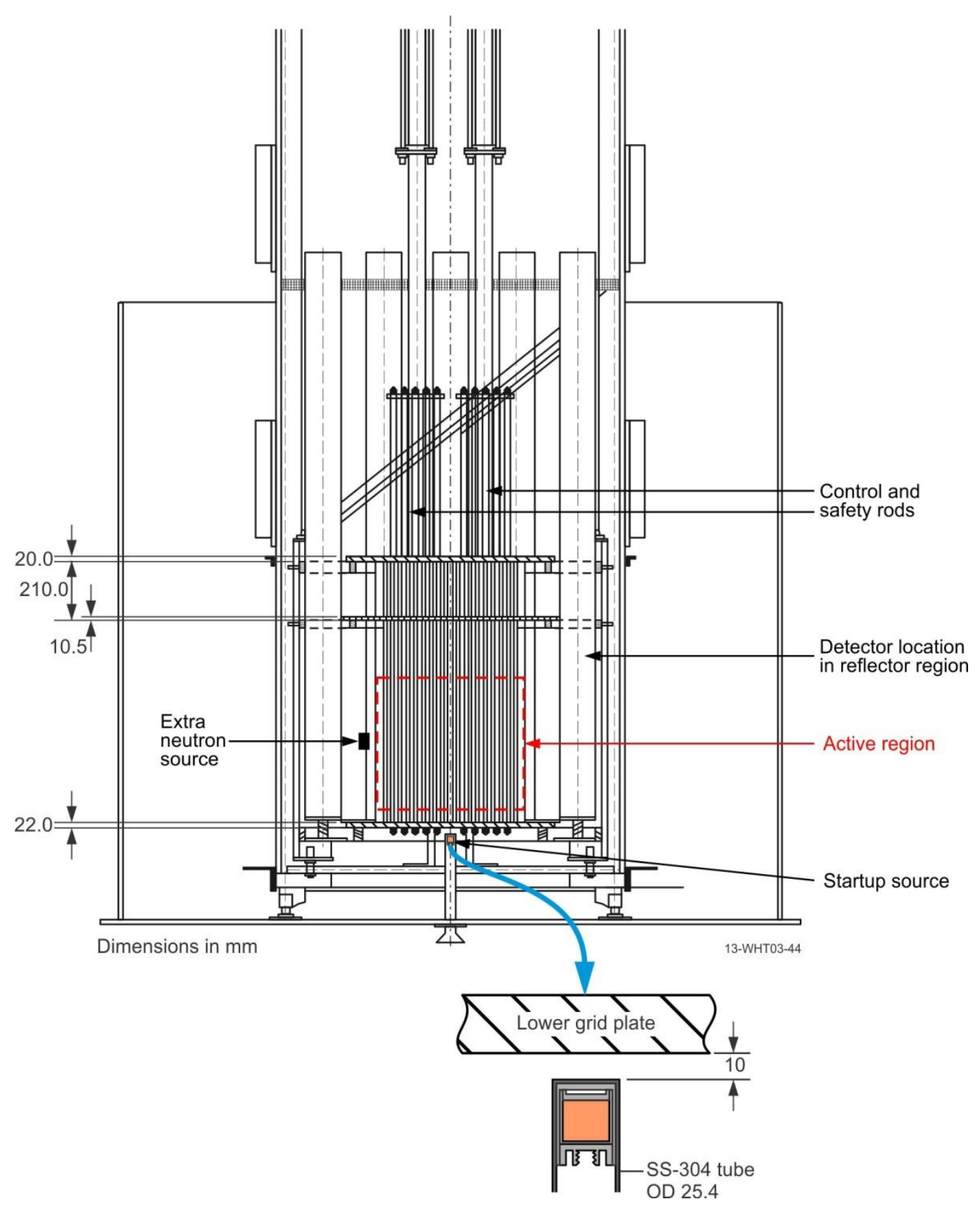

Fig. 3.5. Vista lateral do núcleo do Reator IPEN/MB-01 que mostra as posições dos detectores e das fontes de nêutrons. 


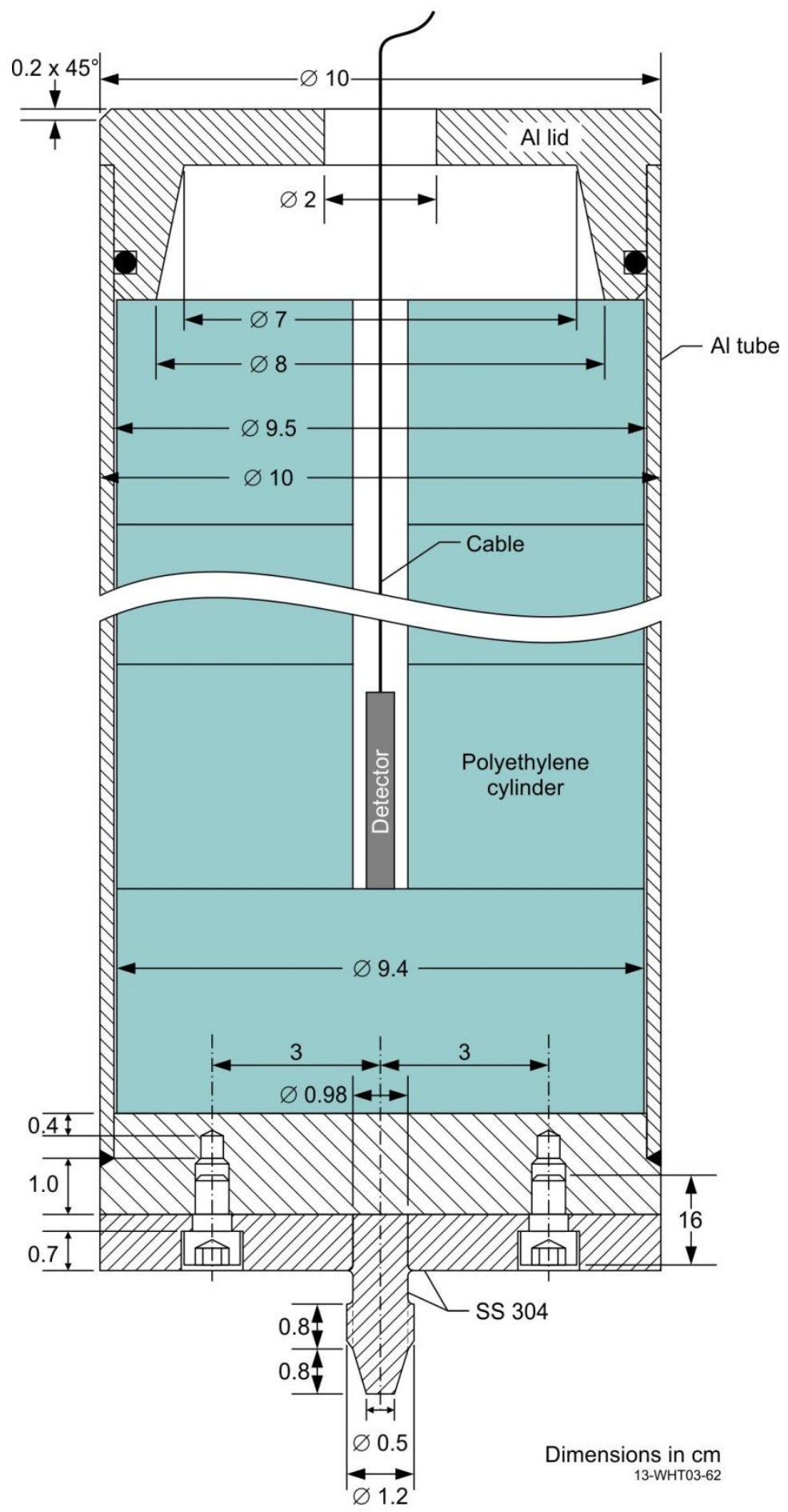

Fig. 3.6. Detalhes do tubo do detector. 


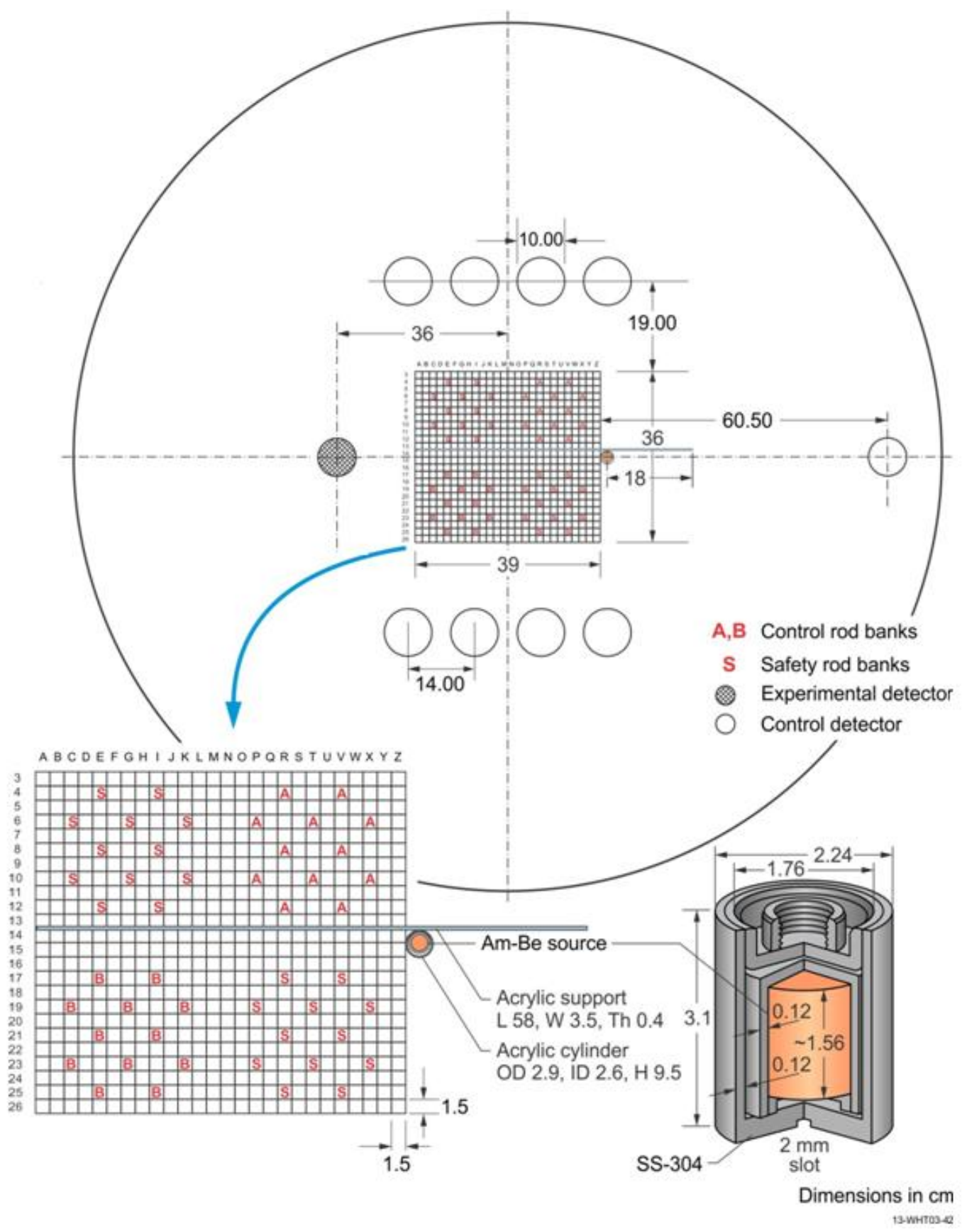

Fig. 3.7. Vista superior do núcleo do reator IPEN/MB-01. 


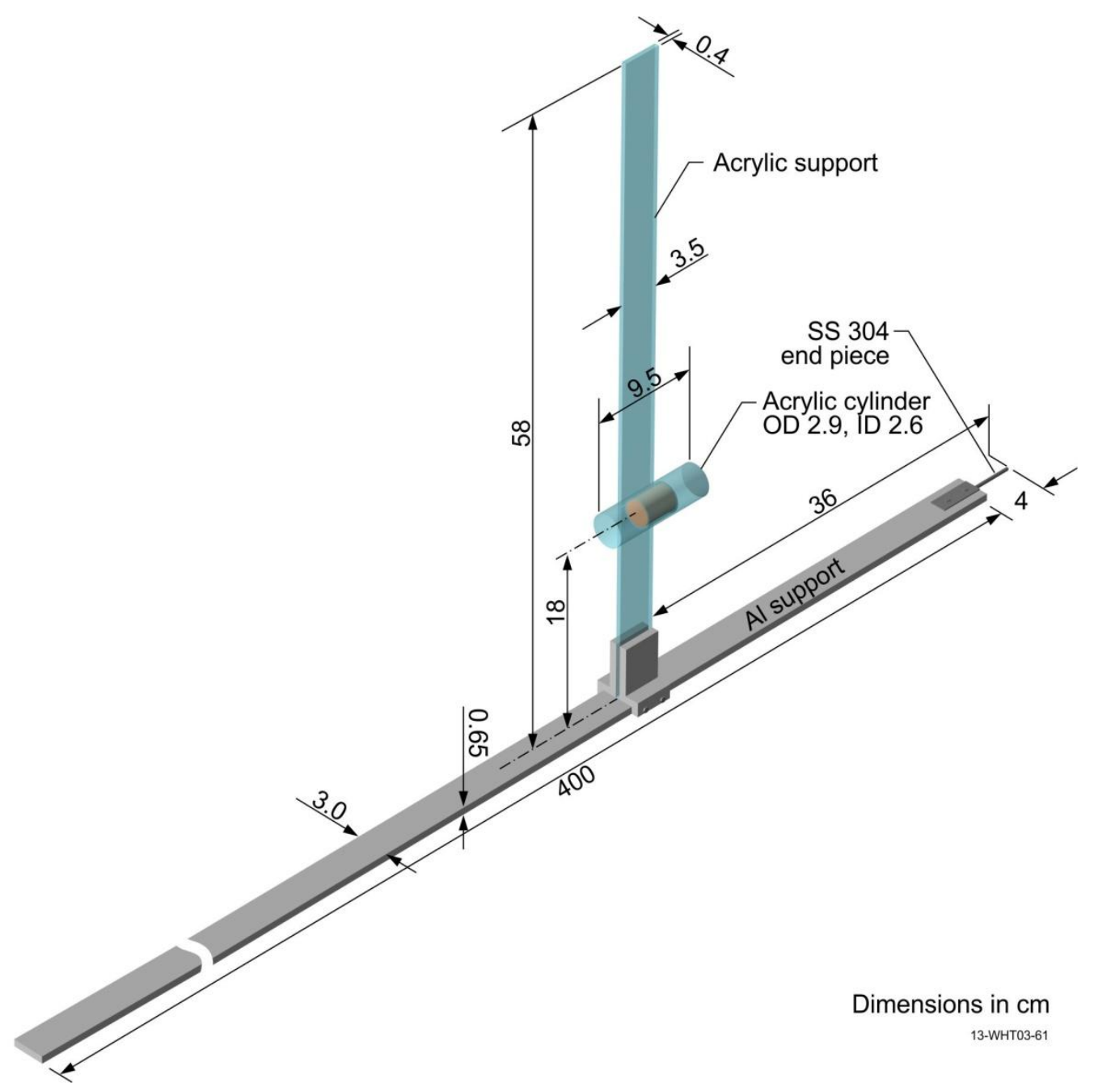

Fig. 3.8. Dados geométricos do suporte da fonte adicional (Am-Be).

\subsubsection{Procedimento Experimental.}

As medidas foram agrupadas em quatro partes, dependendo das posições dos bancos de controle (no sentido da inserção da reatividade negativa), da sensibilidade do detector e da utilização de fontes externa de nêutrons, conforme mostrado na Tabela 3.1.

No domínio da reatividade, os três detectores de modo pulso mencionados na 
seção anterior foram distribuidos da seguinte forma: $\mathrm{o} \mathrm{de} \mathrm{BF}_{3}$, perto da criticalidade (de 0 a -1700 pcm); o de ${ }^{3} \mathrm{He}$ de sensibilidade média, na região central (de $\sim-1900$ a $\sim-3200 \mathrm{pcm}$ ); e o de ${ }^{3} \mathrm{He}$ de sensibilidade alta, na região mais subcrítica (de $\sim-3900$ a $\sim-7500 \mathrm{pcm})$. Para alcançar esta gama de reatividade $(0 \sim-7500 \mathrm{pcm})$, os dois bancos de controle, $\mathrm{BC} 1$ e $\mathrm{BC} 2$, sempre mantidos alinhados, foram inseridos simultaneamente em passos de $5 \%$ ou 2,5\%, dependendo da posição dos bancos. A unidade $\%$ representa a porcentagem do comprimento retirado das varetas em relação ao comprimento total ativo das mesmas, o qual é de $54,6 \mathrm{~cm}$. Ou seja, o sistema tornase cada vez mais subcrítico à medida que a \% diminui. A Fig. 3.9 mostra esquematicamente as posições dos bancos de controle em relação ao comprimento ativo dos combustíveis. O símbolo WD representa posição de retirada dos bancos.

Tabela 3.1. Detectores e fontes de nêutrons adicionais utilizados nos experimentos de APSD.

\begin{tabular}{ccc}
\hline $\begin{array}{c}\text { Posição de BC }(\% \\
\text { retirado })\end{array}$ & Detectores Utilizados & $\begin{array}{c}\text { Fonte Externa de } \\
\text { Nêutrons }\end{array}$ \\
\hline de 93 a 75,5 & $\mathrm{BF}_{3}$ sensibilidade baixa & $100 \mathrm{mCi}$ \\
de 70,5 a 65,5 & $\mathrm{BF}_{3}$ sensibilidade baixa & $1 \mathrm{Ci}$ \\
de 63 a 50,5 & ${ }^{3} \mathrm{He}^{2}$ sensibilidade média & $2 \mathrm{Ci}{ }^{\text {(a) }}$ \\
de 45,5 a 0 & ${ }^{3} \mathrm{He}$ sensibilidade alta & $1 \mathrm{Ci}$ \\
\hline
\end{tabular}

(a) Duas fontes de 1Ci

A medida foi iniciada com os dois bancos de controle na posição de $93 \%$ retirada. Esta posição foi escolhida devido ao compromisso de obter a posição do banco de controle o mais próximo possível à do estado crítico, e também, devido à condição de não saturar a medida do detector, que no caso é o de $\mathrm{BF}_{3}$. Apartir desta posição, os bancos de controle foram inseridos simultaneamente em passos cuidadosamente escolhidos para que as taxas de contagem nos dois estados consecutivos fossem significativamente diferentes, sem que as condições da validade da perturbação de primeira ordem, a qual é a base para o desenvolvimento do modelo 
de Gandini e Salvatores, fossem violadas. Em cada um desses estados subcríticos foram medidos a APSD e a taxa de contagem.

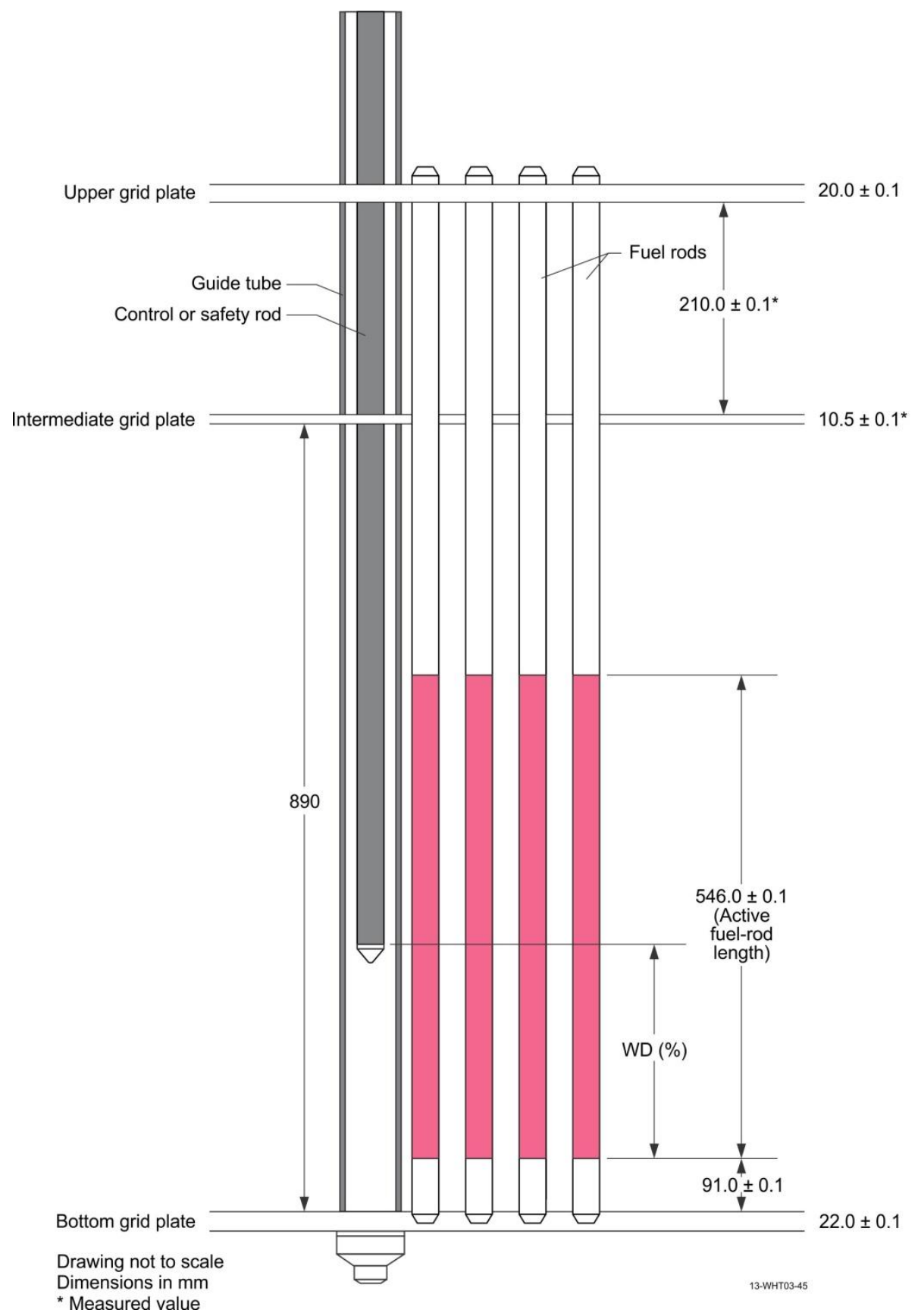

Fig. 3.9. Esquema do mecanismo dos bancos de controle.

Os parâmetros da expressão da potência relativa, $P_{N}$, Eq. (2.20), de dois 
estados subsequentes, devem ser obtidos mantendo tanto as configurações geométricas quanto as propriedades materiais dos detectores e das fontes de nêutrons. Entretanto, houve trocas de detectores durante os experimentos, além de alterações na intensidade das fontes externas de nêutrons. Por esta razão, sempre que houve uma mudança, seja de detector, seja de fonte de nêutrons, medidas nas três posições anteriores à mudança foram realizadas nas condições alteradas. Por exemplo, na Tabela. 3.1, uma vez finalizadas as medidas com o detector $\mathrm{BF}_{3}$ e com a fonte externa de nêutrons de 100 $\mathrm{mCi}$, e depois a fonte foi substituída por uma de $1 \mathrm{Ci}$, as medidas nas posições $85 \%$, $80,5 \%$ e $75,5 \%$ foram realizadas com a fonte de 1 Ci. Desta maneira, a potência relativa na primeira posição após a mudança, a de 70,5\%, foi calculada a partir dos parâmetros obtidos na posição de 75,5\% e 70,5\%, na nova condição. As repetições de medidas nas posições de $85 \%$ e $80,5 \%$ foram realizadas a fim de comparar os valores dos parâmetros finais, a saber, $\rho_{g e n} \mathrm{e} \zeta$, nas duas configurações diferentes, e avaliar a adequabilidade deste procedimento. $\mathrm{O}$ mesmo procedimento foi repetido nas posições de $63 \%$ e de $45,5 \%$.

A temperatura na região do combustível foi monitorizada por meio de um conjunto de termopares, estrategicamente localizados na região ativa do núcleo do reator. Os termopares são feitos de uma liga de $\mathrm{Cu}-\mathrm{Ni}$ ( $55 \% \mathrm{Cu}$ e $45 \%$ de Ni) e o seu diâmetro é de 1,6 mm. No total, 12 termopares foram utilizados neste experimento como em LEU.COMP.THERM.077 (Dos Santos, et al., 2004a). A Fig. 3.10 mostra as posições horizontais de tais termopares. Em relação ao núcleo ativo do reator, os termopares T1, T2, T3 e T4 estão posicionados na parte inferior; os T5, T6, T7 e T8, do meio; e os T9, T10, T11 e T12, na parte superior. Os termopares foram calibrados por um procedimento padrão, e a precisão exigida para as medidas absolutas é de \pm $0,02{ }^{\circ} \mathrm{C}$. A temperatura média de todo o conjunto de experiências foi de $\mathbf{1 9 , 6}(\mathbf{\pm 0 , 2}){ }^{\circ} \mathbf{C}$ $(1 \sigma)$. A reatividade total devida a tais termopares foi medida sendo $-12.5( \pm 2.0) \mathrm{pcm}$.

A incerteza no posicionamento dos bancos de controle é relacionada principalmente com o estabelecimento do seu nível de referência, bem como a 
linearidade do sistema de aquisição. A linearidade do sistema de banco de controle é verificada rotineiramente e tem sido provado ser adequado para os fins do experimento. A incerteza no posicionamento do banco de controle devido principalmente à exatidão do padrão da mecânica para definir o nível de referência. Esta incerteza é igual a $0,1 \mathrm{~mm}$, o que representa menos de $1,0 \mathrm{pcm}$ e pode ser desprezada em toda a análise de incerteza do experimento.

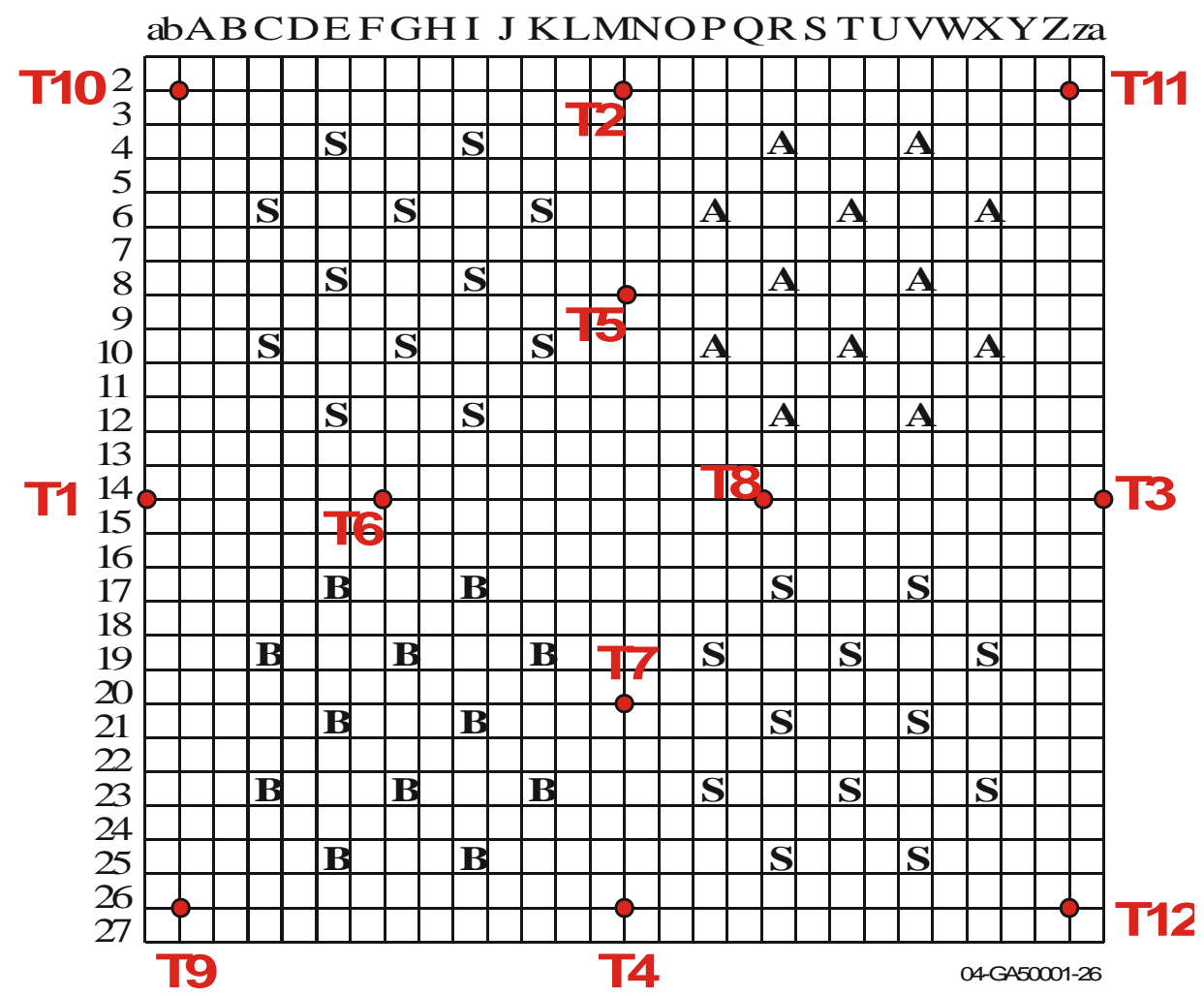

Fig. 3.10. Posição horizontal dos Termopares na configuração padrão (28 x26) do reator IPEN/MB-01. Os T1, T2, T3 e T4 são os inferiores; os T5, T6, T7 e T8, do meio; e, os T9, $\mathrm{T} 10, \mathrm{~T} 11$ e T12, os superiores.

\subsubsection{Experimento da CPSD}

No experimento da CPSD, além dos três detectores do experimento da APSD, foi utilizado mais um detector de $\mathrm{BF}_{3}$ com as mesmas especificações técnicas e do 
mesmo fabricante do detector $\mathrm{BF}_{3}$ anterior. Os detectores foram emparelhados da seguinte forma: um par formado por um detector de $\mathrm{BF}_{3}$ e o de ${ }^{3} \mathrm{He}$ de sensibilidade alta; e outro par, formado por outro detector de $\mathrm{BF}_{3}$ e o de ${ }^{3} \mathrm{He}$ de sensibilidade média. Os detectores foram posicionados na face oeste do núcleo do reator conforme ilustrado na Fig. 3.11, ambos alinhados a uma distância de $15 \mathrm{~cm}$ das varetas de combustíveis, aproximadamente. A distância entre os eixos dos detectores foi de $28 \mathrm{~cm}$.

O experimento das medidas de CPSD foi dividido em três partes, como mostrado na Tabela 3.2. Da posição de $93 \%$ à de $73 \%$, foram utilizados dois detectores de $\mathrm{BF}_{3}$ da mesma especificação técnica e não foi instalada fonte externa de nêutrons. Na posição de 70,5\%, os dois detectores foram trocados por dois de ${ }^{3} \mathrm{He}$ : um de sensibilidade alta e outro, de sensibilidade média, e uma fonte adicional de 1Ci foi utilizada a partir da posição de $63 \%$, até chegar à de $40,5 \%$. A posição da fonte foi a mesma do experimento anterior. Neste experimento, como no experimento da APSD anterior, foram realizadas medidas nas três posições anteriores a qualquer mudança na configuração, seja do detector ou da fonte adicional.

Os passos de inserção dos BCs foram mantidos em 2,5\% em todos os casos, diferente do experimento da APSD, no qual inserções tinham passos de 2,5 ou 5,0\%.

Tabela 3.2. Detectores e fontes de nêutrons adicionais utilizados nos experimentos de CPSD.

\begin{tabular}{ccc}
\hline $\begin{array}{c}\text { Posição de BC } \\
\text { (\% retirado) }\end{array}$ & Detectores Utilizados & $\begin{array}{c}\text { Fonte Externa de } \\
\text { Nêutrons }\end{array}$ \\
\hline 93 a 73,0 & Dois de $\mathrm{BF}^{3}$ sensibilidade baixa & sem fonte externa \\
70,5 a 65,5 & ${ }^{3} \mathrm{He}$ sensibildade média e alta & sem fonte externa \\
63 a 40,5 & ${ }^{3} \mathrm{He}$ sensibildade média e alta & $1 \mathrm{Ci}$ \\
\hline
\end{tabular}




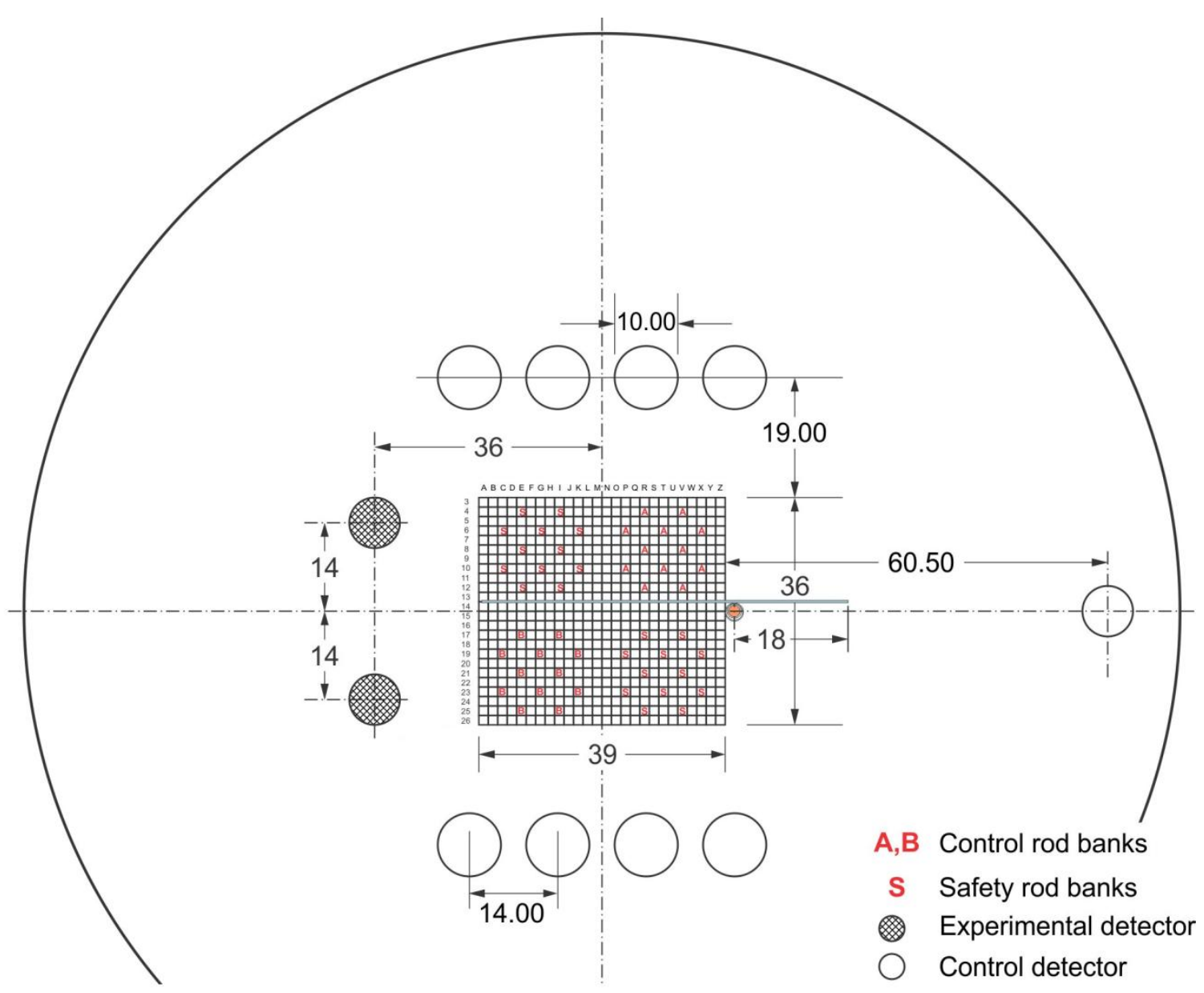

Fig. 3.11. Posições dos detectores e da fonte externa de nêutrons nas medidas de CPSD.

Além da CPSD, foram obtidas duas APSDs, cada uma associada a um par dos detectores.

Os detalhes dos procedimentos experimentais se encontram no Apêndice C. 


\section{PROCESSAMENTOS DE SINAIS E AQUISIÇÃO DE DADOS}

O método experimental é baseado em equipamentos virtuais utilizando placas de aquisição de dados juntamente com a linguagem gráfica LabVIEW. Os pulsos oriundos dos detectores modo pulso são adquiridos pela placa de aquisição de dados e transformados em linguagem digital para a leitura e interpretação do software LabVIEW.

Um diagrama de blocos da cadeia eletrônica de processamento de sinais e do sistema de aquisição de dados está ilustrado na Fig. 4.1. De acordo com a figura, pulsos de nêutrons provenientes de detectores são formatados e amplificados por préamplificadores e amplificadores e, subsequentemente discriminados da radiação- $\gamma$ através de módulos monocanais (Single-Channel Analyzer - SCA) (Naing, 2004). Nas saídas dos monocanais são gerados pulsos lógicos negativos (padrão NIM fast negative) (Naing, 2004) com largura de 25ns e amplitude de $-5 \mathrm{~V}$ sobre uma

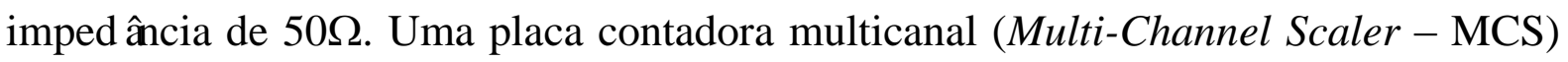
registra pulsos lógicos em pequenos intervalos de tempo, chamados dwell time. Este procedimento é totalmente análogo ao trabalho do Kitamura (Kitamura, 1999). O valor mínimo para o dwell time do MCS é de 100ns e o número de canais pode variar de 4 a 65536. O dwell time determina a frequência máxima a ser analisada; e o número de canais, a resolução da frequência correspondente. Além disso, esta placa possui memória tipo dual-port a qual permite acesso direto aos dados espectrais para fins de processamento e análise, sem que a aquisição seja interrompida.

De modo a garantir uma taxa de aquisição de dados satisfatória, minimizando o tempo morto de processamento, as características do PC de aquisição são de suma importância. Por esta razão, a placa MCS está instalada em um PC com processador de $3.0 \mathrm{GHz}$ o qual é dedicado exclusivamente à aquisição de dados. 
O controle da aquisição de dados é realizado através de instrumentos virtuais (Virtual Instruments-VI's), desenvolvidos em linguagem G (Graphical programming language) utilizando o software LabVIEW 5.1 (Laboratory Virtual Instrument Engineering Workbench) da National Instruments sob uma plataforma Windows XP.

Um segundo PC (processador de 3.0GHz), para o qual os dados são transferidos via comunicação ethernet (protocolo TCP/IP), é utilizado para o processamento dos dados. O processamento e análise dos dados são realizados via códigos $\mathrm{C} / \mathrm{C}++$ sobre uma plataforma Windows XP. Desta forma, o IPEN/MB-01 correlator proporcionará uma análise on-line das densidades espectrais obtidas com detectores modo pulso.

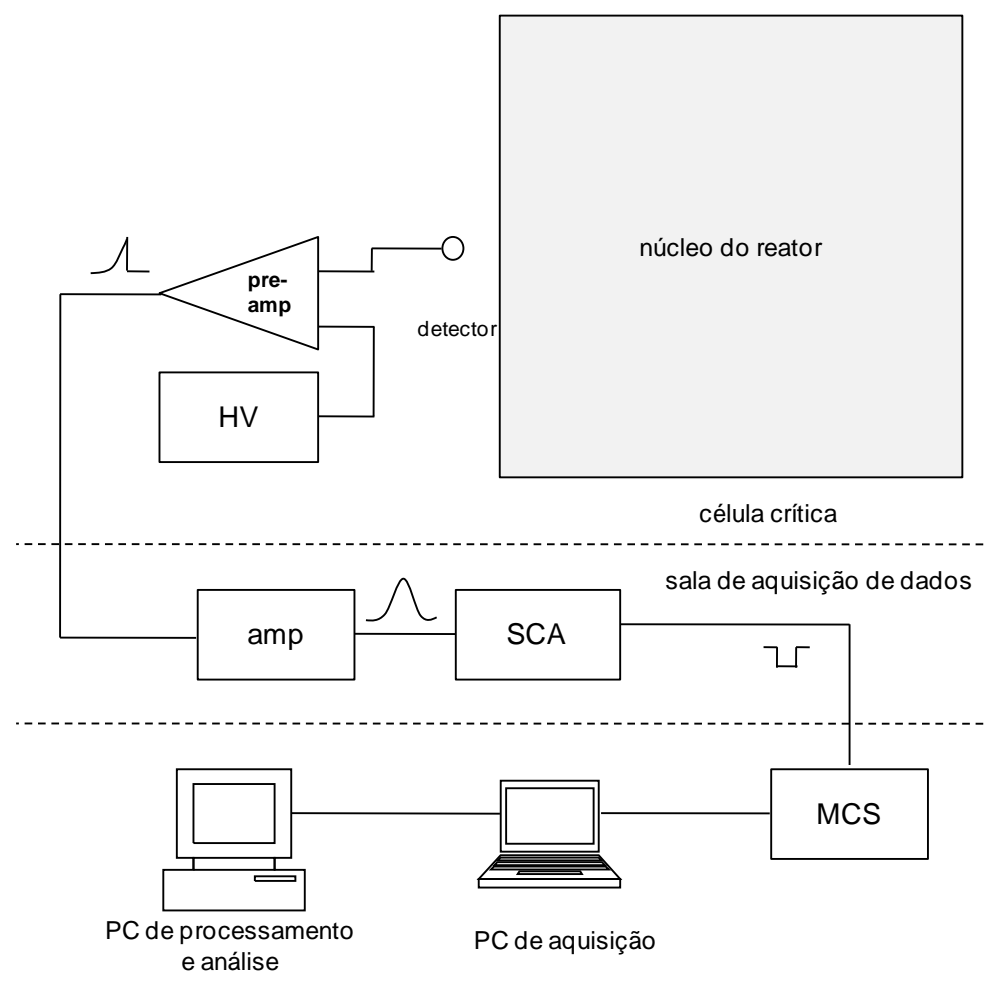

Fig. 4.1. Diagrama de blocos da cadeia eletrônica e do sistema de aquisição e processamento de dados IPEN/MB-01 utilizado em medidas de APSD. 
A Fig. 4.2 mostra o esquema de aquisição de dados no domínio de tempo pela placa de MCS para processamento e obtenção das densidades espectrais, neste caso a APSD.

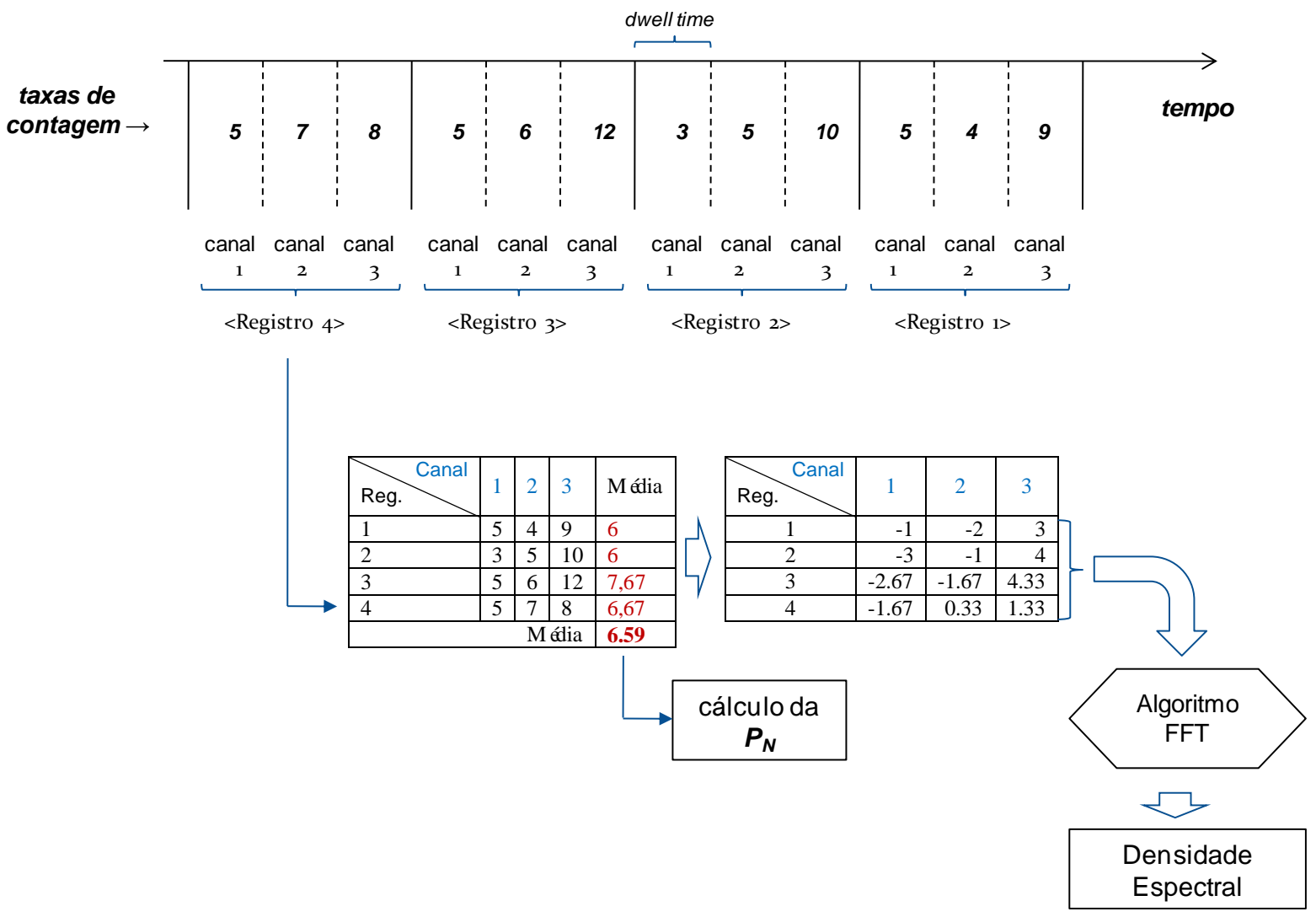

Fig. 4.2. Ilustração do esquema de processamento de dados para obtenção de densidade espectral. Um caso particular para ilustração, com 4 medidas e 3 canais.

Primeiro, faz-se a aquisição das contagens do detector; elas distribuem-se em canais da mesma largura (dwell times), previamente definido pelo usuário. As contagens por dwell time resulta em taxas de contagem, $R$. Uma vez terminada a aquisição, calcula-se o valor médio das taxas de contagem de cada registro. Em seguida, taxa de contagem de cada canal é subtraída pelo valor médio, resultando apenas nas flutuações em torno do valor médio. Finalmente, realiza-se o procedimento de Transformada de Fourier Rápida ou FFT (Fast Fourier Transform) (Agilent 
Technologies, 2000) para poder obter as densidades espectrais. FFT é um algoritmo para computação de Transformada de Fourier Discreta. As taxas de contagem a serem utilizadas para calcular potência relativa pela Eq. (2.20) ou Eq. (2.21) são dadas pela média de $R$ médios ao longo dos registros.

\subsection{APSD}

A Fig. 4.3 mostra duas APSDs típicas, obtidas em um estado perto do crítico, e em estado subcrítico de quase $-2500 \mathrm{pcm}$. Os números de médias foram de 500 e de 870, respectivamente. Deve-se notar que as escalas utilizadas para a representação desses espectros são logarítmicas.

Pode-se notar que, existe um intervalo da frequência no qual a APSD é constante. Esse plateau ocorre devido à presença dos nêutrons atrasados. Além desse primeiro plateau, existe um segundo à direita dos gráficos, devido ao processo aleatório de deteç̧ão de nêutrons e é conhecido como plateau devido ao ruído não correlacionado. Esse ruído não correlacionado é eliminado quando se faz a correlação cruzada, ou CPSD.

O dwell time foi ajustado para $2 \cdot 10^{-4}$ segundos, que resulta em frequência máxima de $2.5 \mathrm{kHz}$ (single-sided spectrum), e o número de canais escolhido, no domínio de tempo, é de 8192, que dá resolução no domínio de frequência de cerca de $0.61 \mathrm{~Hz}$. O tamanho do registro - o tempo necessário para concluir uma aquisição é (2 x $\left.10^{-4}\right) \cdot(8192) \cong 1.64 \mathrm{~s}$. Cada ponto experimental da APSD tem uma barra de erro dado por $N^{1 / 2}(\%)$, onde $N$ é o número de médias (Bendat e Piersol, 2000). Em geral, o número de médias varia de 500 a 1.000. 


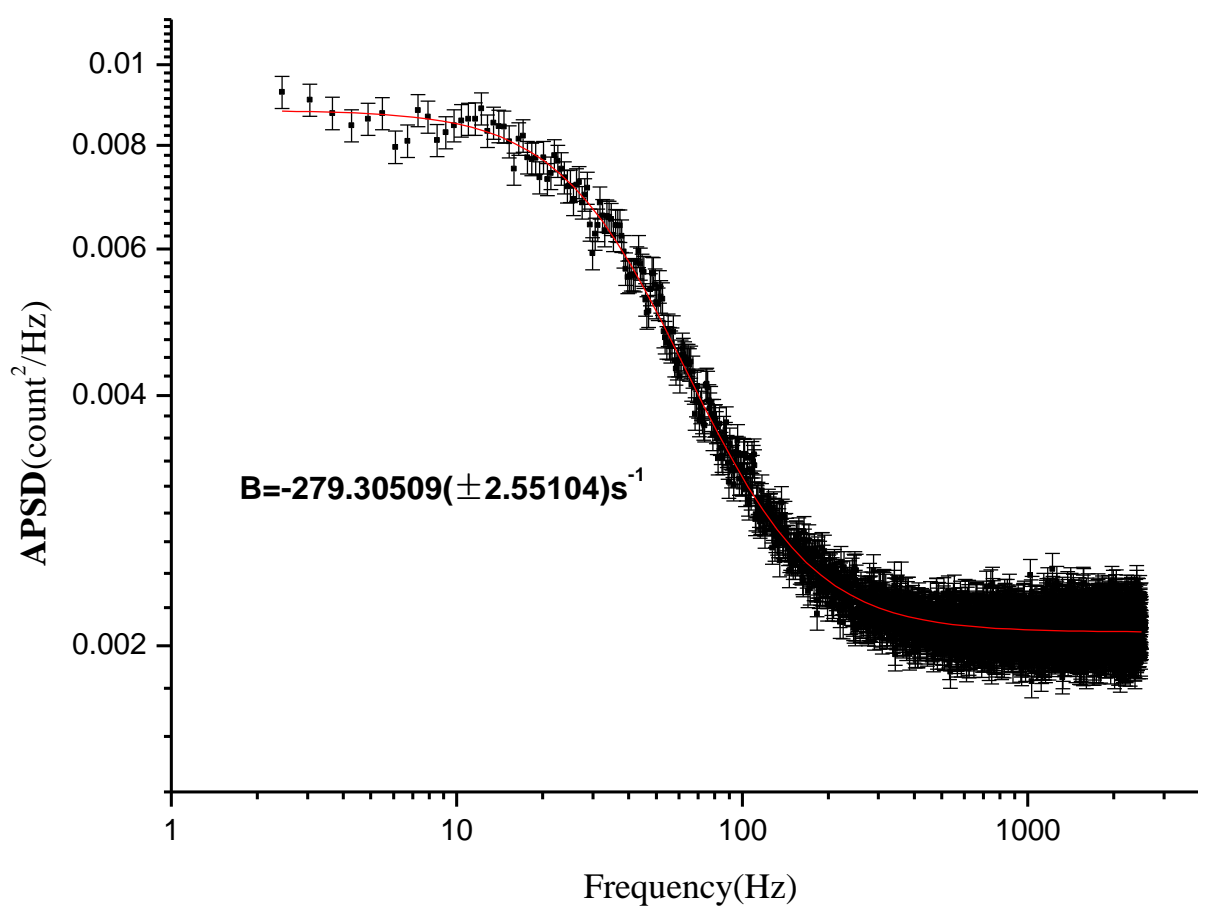

(a)

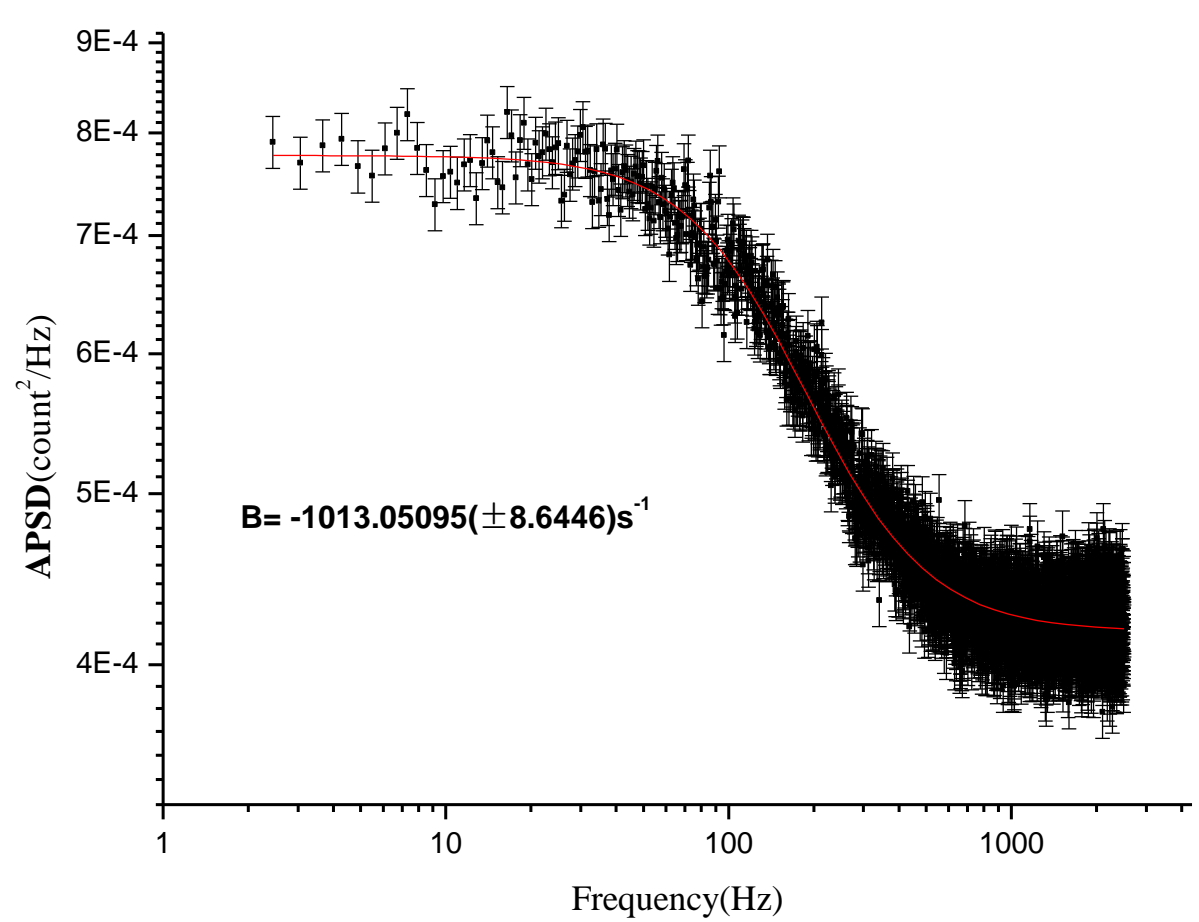

(b)

Fig. 4.3. APSD experimental obtida num estado perto do crítico (a) e em um estado subcrítico $(-2500 \mathrm{pcm})(\mathrm{b})$. A linha vermelha sólida é o ajuste de mínimos quadrados através da Eq. (2.14). 
Como pode ser visto na Fig. 4.3, as APSDs mostram o comportamento esperado da reatividade. $\mathrm{O}$ valor absoluto do $B$, que é o mesmo que o $\alpha$ dado por ( $\rho$ $\beta) / l_{\text {eff }}$ no modelo da cinética pontual clássica, aumenta à medida que a reactividade negativa aumenta, e a diferença entre o ruído correlacionado - primeiro plateau - e o não correlacionado - segundo plateau - diminui. Além disso, a reta da inclinação desloca-se para a direita do gráfico. Nota-se, também, que a dispersão de dados é maior no caso mais subcrítico. A dispersão em alguns estados subcríticos pode ser reduzida utilizando fontes de nêutrons adicionais, conforme descrito na próxima seção. Pode-se concluir que, apesar das flutuações maiores em alguns estados subcríticos, os dados são de boa qualidade, e que representam os aspectos físicos do problema.

\subsection{CPSD}

Para o experimento da CPSD, o dwell time foi ajustado a $1.25 \cdot 10^{-4}$ segundos, o que resultou na frequência máxima a ser analisada igual a $4 \mathrm{kHz}$. O número de canais escolhido foi 8000; a resolução no domínio da frequência, $\Delta f, 1 \mathrm{~Hz}$; e o número de médias foi 1000 em todos os casos.

A Fig. 4.4. ilustra a cadeia da eletrônica e do sistema de aquisição de dados, utilizados no experimento da CPSD. Neste caso, os sinais provêm dos dois detectores e são processados em SCA.

Com dois detectores pode-se obter a APSD de cada um deles e, ao mesmo tempo, a CPSD, que corresponde ao cruzamento dos sinais dos dois detectores. Em geral, densidade espectral do tipo CPSD fornece uma medida do quanto dois sinais distintos estão correlacionados por um agente comum. 


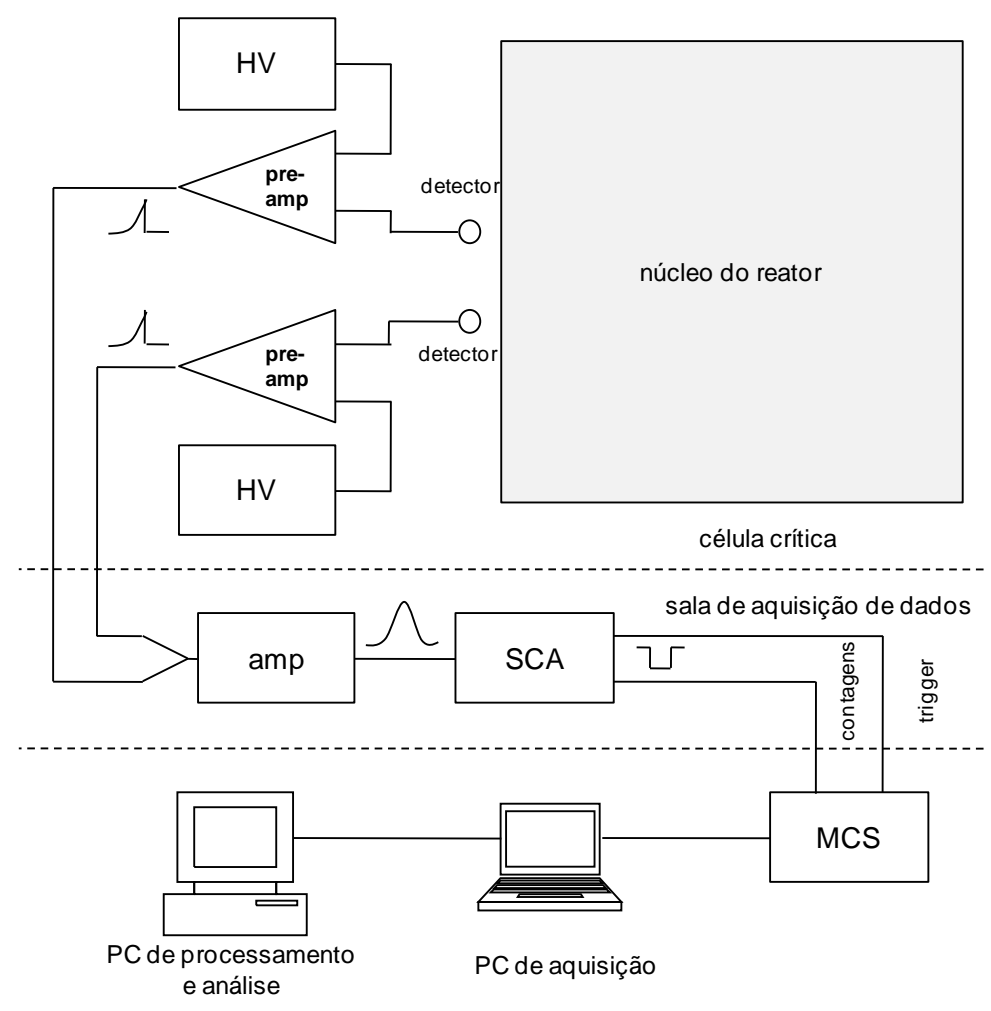

Fig. 4.4. Diagrama de blocos da cadeia eletrônica e do sistema de aquisição e processamento de dados do IPEN/MB-01 utilizado em medidas de CPSD.

A Fig. 4.5. mostra duas CPSDs, uma do estado crítico e outra de um nível subcrítico que corresponde à reatividade de -2613 pcm. Pode-se notar que, neste caso, não há o patamar (ou plateau) inferior como no caso da APSD, e conseqüentemente, o ajuste de mínimos quadrados é feita por uma função diferente. Isso, porque, com a CPSD, o componente relacionado com o ruído não correlacionado é eliminado. Além disso, o ajuste da curva não é levado até a frequência máxima, mas foi restringido no intervalo onde não apresenta dispersão considerável dos pontos. 


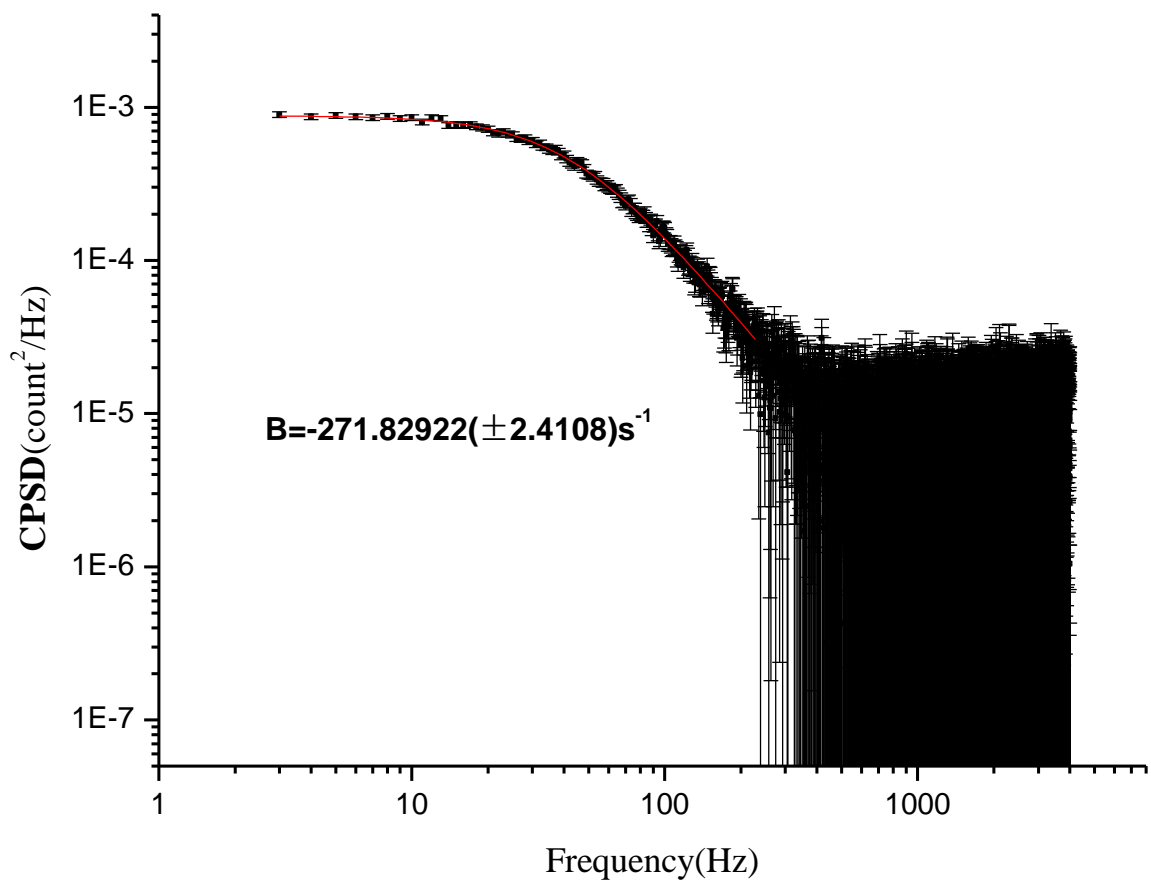

(a)

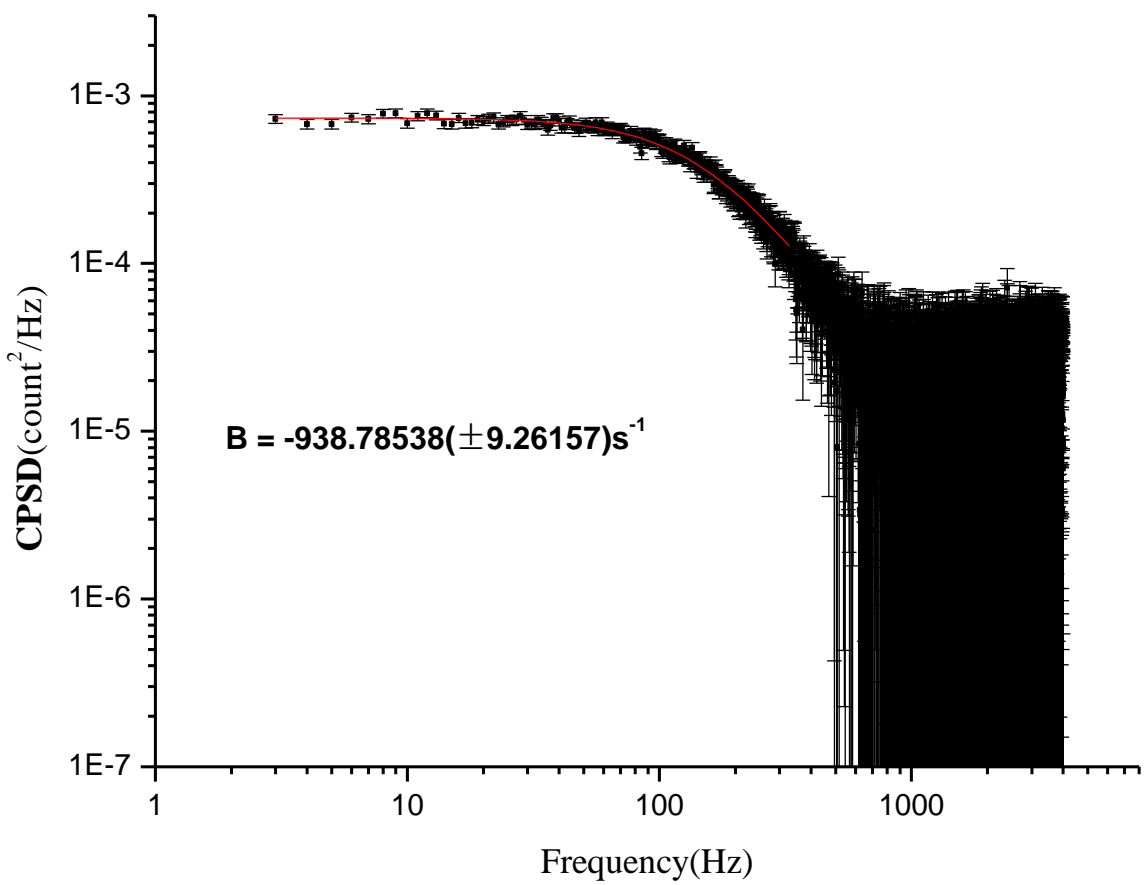

(b)

Fig. 4.5. CPSD experimental obtida num estado perto do crítico (a) e em um estado subcrítico $(-2615$ pcm) (b). A linha vermelha sólida é o ajuste de mínimos quadrados através da Eq. (2.22). 


\section{RESULTADOS EXPERIMENTAIS}

\subsection{Os resultados da APSD}

Os resultados das medidas consistem em um conjunto de contagens no detector, APSD e temperatura para cada configuração analisada neste trabalho. A Tabela 5.1 mostra as contagens medidas no detector para cada configuração. Como já foi mencionado, as posições expressas em unidade de porcentagem retirada referem-se aos dois bancos de controle: $\mathrm{BC} 1$ e $\mathrm{BC} 2$; ambos deslocando-se sempre alinhados e simultaneamente.

O tratamento das medidas subcríticas começa com as APSDs. Aos pontos experimentais da APSD obtida a cada configuração subcrítica foi ajustada uma curva da Eq. (2.11), pelo método dos mínimos quadrados, e assim, foram obtidos os parâmetros $B$ e $C$ na Eq. (2.20). Os ajustes foram feitos com os primeiros quatro pontos das APSDs excluídos do conjunto de dados por serem atípicos; a saber, os que são associados às frequências: 0.0000 Hz, 0.6104 Hz, $1.2207 \mathrm{~Hz}$ e $1.8311 \mathrm{~Hz}$.

O plateau superior, $\Phi^{p}$, na Eq. (2.20), obteve-se pela média dos valores da APSD na região do patamar superior, à esquerda, do gráfico na Fig. 4.3. Este procedimento mostrou-se mais satisfatório do que efetuar o ajuste dos mínimos quadrados, visto que resultou nas melhores resoluções dos valores de $\zeta$ e $\Sigma \rho_{g e n}$. O intervalo da frequência no qual foi extraída a média varia em cada caso. A Tabela 5.2 mostra os valores de $B, C$ e $\Phi^{p}$, e suas respectivas incertezas, para cada configuração.

Com isso, a potência relativa (ou normalizada), $P_{N}$, é determinada para cada estado subcrítico em relação ao estado imediatamente anterior pela Eq. (2.20), e, subsequentemente, os respectivos $\zeta$ e $\rho_{\text {gen }}$ são calculados por Eq. (2.17) e Eq. (2.18), respectivamente. A reatividade de um estado em relação ao estado inicial, i.e., de $93 \%$ 
retirado, foi considerada como sendo a soma de $\rho_{\text {gen }}$ parciais entre ambos os estados incluindo o $\rho_{\text {gen }}$ do estado em questão, e é denotada neste trabalho por $\Sigma \rho_{g e n}$. A Tabela 5.3 apresenta os valores desses três parâmetros deduzidos: $P_{N}, \rho_{g e n} \mathrm{e} \zeta$. Os valores de $\beta_{\text {eff }}$ e $l_{\text {eff, }}$ utilizado nesta abordagem experimental, foram extraídos do manual do IRPhE (Dos Santos, 2009), e esses valores são benchmarks internacionais realizados no reator IPEN/MB-01 para a determinação dos parâmetros efetivos de nêutrons atrasados. Os valores utilizados no experimento foram $0,00750( \pm 0,00005)$ e $32,02( \pm 1.06) \mu \mathrm{seg}$, respectivamente, para $\beta_{\text {eff }}$ e $l_{\text {eff }}$.

Tabela 5.1: Contagens de detector em várias configurações.

\begin{tabular}{|c|c|c|c|c|}
\hline $\begin{array}{c}\text { Posição } \\
\text { dos BCs (\%) }\end{array}$ & Detector & $\begin{array}{l}\text { Fonte extra } \\
\text { de nêutrons }\end{array}$ & Contagem, $R$ & $\sigma_{\mathbf{R}}^{(\mathbf{a})}$ \\
\hline 93,0 & \multirow{10}{*}{$\begin{array}{c}\mathrm{BF}_{3} \\
\text { (sensibilidade baixa) }\end{array}$} & \multirow{8}{*}{$100 \mathrm{mCi}$} & 30607,45 & 13,71 \\
\hline 90,5 & & & 20851,88 & 10,87 \\
\hline 88,0 & & & 15230,18 & 9,36 \\
\hline 85,5 & & & 11567,67 & 7,17 \\
\hline 83,0 & & & 9125,26 & 5,89 \\
\hline 80,5 & & & 7363,52 & 4,75 \\
\hline 78,0 & & & 6075,10 & 4,09 \\
\hline 75,5 & & & 5098,04 & 3,00 \\
\hline 70,5 & & \multirow{2}{*}{$1 \mathrm{Ci}$} & 11556,28 & 3,42 \\
\hline 65,5 & & & 8739,06 & 2,92 \\
\hline 63,0 & \multirow{4}{*}{$\begin{array}{c}{ }^{3} \mathrm{He} \\
\text { (sensibilidade média) }\end{array}$} & \multirow{4}{*}{$2 \mathrm{Ci}$} & 36397,80 & 7,37 \\
\hline 60,5 & & & 32881,35 & 7,23 \\
\hline 58,0 & & & 29731,79 & 6,68 \\
\hline 55,5 & & & 26936,41 & 6,29 \\
\hline 53,0 & \multirow{14}{*}{$\begin{array}{c}{ }^{3} \mathrm{He} \\
\text { (sensibilidade alta) }\end{array}$} & \multirow{14}{*}{$1 \mathrm{Ci}$} & 24457,73 & 5,87 \\
\hline 50,5 & & & 22719,02 & 5,61 \\
\hline 45,5 & & & 26148,07 & 5,30 \\
\hline 43,0 & & & 24278,94 & 5,16 \\
\hline 40,5 & & & 22467,38 & 4,29 \\
\hline 35,5 & & & 19533,19 & 3,54 \\
\hline 33,0 & & & 18545,55 & 4,11 \\
\hline 30,5 & & & 17367,21 & 3,20 \\
\hline 25,5 & & & 15765,28 & 3,10 \\
\hline 20,5 & & & 14594,52 & 2,96 \\
\hline 15,5 & & & 13775,57 & 2,78 \\
\hline 10,5 & & & 13197,45 & 2,75 \\
\hline 5,5 & & & 12831,92 & 2,65 \\
\hline 0,0 & & & 12601,03 & 2,61 \\
\hline
\end{tabular}

${ }^{(a)}$ Desvio padrão da média 
Tabela 5.2: Os parâmetros $B, C$ e $\Phi^{p}$ para determinação dos níveis subcríticos

\begin{tabular}{|c|c|c|c|c|c|c|}
\hline $\begin{array}{c}\text { Posição } \\
\text { dos BCs } \\
(\%)\end{array}$ & $\mathbf{B}\left(\mathrm{s}^{-1}\right)$ & $\sigma_{\mathrm{B}}\left(\mathrm{s}^{-1}\right)^{(a)}$ & $\Phi^{\mathbf{p}(\mathbf{b})}$ & $\boldsymbol{\sigma}_{\boldsymbol{\Phi} \mathbf{p}}{ }^{(\mathbf{b}),(\mathbf{c})}$ & $\mathbf{C}^{(\mathbf{b})}$ & $\sigma_{\mathbf{C}}^{(a),(b)}$ \\
\hline 93,0 & $-279,305$ & 2,551 & $2,080 \mathrm{E}-03$ & $1,584 \mathrm{E}-06$ & $8,620 \mathrm{E}-03$ & $1,576 \mathrm{E}-04$ \\
\hline 90,5 & $-304,859$ & 2,657 & $1,490 \mathrm{E}-03$ & $1,089 \mathrm{E}-06$ & $5,740 \mathrm{E}-03$ & $1,128 \mathrm{E}-04$ \\
\hline 88,0 & $-326,152$ & 2,938 & $1,120 \mathrm{E}-03$ & $8,367 \mathrm{E}-07$ & $4,080 \mathrm{E}-03$ & $4,621 \mathrm{E}-05$ \\
\hline 85,5 & $-359,425$ & 3,242 & $8,700 \mathrm{E}-04$ & $6,289 \mathrm{E}-07$ & $2,790 \mathrm{E}-03$ & $3,993 \mathrm{E}-05$ \\
\hline 83,0 & $-384,844$ & 3,535 & $6,900 \mathrm{E}-04$ & $4,990 \mathrm{E}-07$ & $2,090 \mathrm{E}-03$ & $1,909 \mathrm{E}-05$ \\
\hline 80,5 & $-427,427$ & 4,137 & $5,600 \mathrm{E}-04$ & $4,027 \mathrm{E}-07$ & $1,520 \mathrm{E}-03$ & $2,332 \mathrm{E}-05$ \\
\hline 78,0 & $-462,838$ & 4,712 & 4,700E-04 & 3,339E-07 & $1,120 \mathrm{E}-03$ & $1,372 \mathrm{E}-05$ \\
\hline 75,5 & $-505,884$ & 5,005 & $3,900 \mathrm{E}-04$ & $2,563 \mathrm{E}-07$ & $8,672 \mathrm{E}-04$ & $6,798 \mathrm{E}-06$ \\
\hline 70,5 & $-600,589$ & 5,825 & 8,700E-04 & $4,755 \mathrm{E}-07$ & $1,570 \mathrm{E}-03$ & $9,026 \mathrm{E}-06$ \\
\hline 65,5 & $-703,375$ & 8,240 & $6,600 \mathrm{E}-04$ & $3,787 \mathrm{E}-07$ & $1,060 \mathrm{E}-03$ & $9,106 \mathrm{E}-06$ \\
\hline 63,0 & $-738,132$ & 6,720 & $2,320 \mathrm{E}-03$ & $1,621 \mathrm{E}-06$ & 4,740E-03 & $5,375 \mathrm{E}-05$ \\
\hline 60,5 & $-803,337$ & 8,333 & $2,130 \mathrm{E}-03$ & $1,645 \mathrm{E}-06$ & $4,070 \mathrm{E}-03$ & $3,502 \mathrm{E}-05$ \\
\hline 58,0 & $-873,560$ & 9,099 & $1,960 \mathrm{E}-03$ & $1,499 \mathrm{E}-06$ & $3,650 \mathrm{E}-03$ & $2,546 \mathrm{E}-05$ \\
\hline 55,5 & $-923,480$ & 9,788 & $1,810 \mathrm{E}-03$ & $1,340 \mathrm{E}-06$ & $3,160 \mathrm{E}-03$ & $1,839 \mathrm{E}-05$ \\
\hline 53,0 & $-981,428$ & 11,001 & $1,670 \mathrm{E}-03$ & $1,239 \mathrm{E}-06$ & $2,730 \mathrm{E}-03$ & $2,653 \mathrm{E}-05$ \\
\hline 50,5 & $-1019,118$ & 11,268 & $1,570 \mathrm{E}-03$ & $1,033 \mathrm{E}-06$ & $2,530 \mathrm{E}-03$ & $1,529 \mathrm{E}-05$ \\
\hline 45,5 & $-1270,382$ & 6,854 & $1,750 \mathrm{E}-03$ & $1,177 \mathrm{E}-06$ & $3,900 \mathrm{E}-03$ & $3,245 \mathrm{E}-05$ \\
\hline 43,0 & $-1353,533$ & 7,540 & $1,640 \mathrm{E}-03$ & $1,119 \mathrm{E}-06$ & $3,520 \mathrm{E}-03$ & $1,651 \mathrm{E}-05$ \\
\hline 40,5 & $-1437,141$ & 7,594 & $1,540 \mathrm{E}-03$ & $9,591 \mathrm{E}-07$ & $3,120 \mathrm{E}-03$ & $1,594 \mathrm{E}-05$ \\
\hline 35,5 & $-1572,093$ & 8,528 & $1,360 \mathrm{E}-03$ & $8,185 \mathrm{E}-07$ & $2,530 \mathrm{E}-03$ & $1,184 \mathrm{E}-05$ \\
\hline 33,0 & $-1629,958$ & 10,971 & $1,300 \mathrm{E}-03$ & $9,387 \mathrm{E}-07$ & $2,330 \mathrm{E}-03$ & $9,743 \mathrm{E}-06$ \\
\hline 30,5 & $-1703,513$ & 9,661 & $1,230 \mathrm{E}-03$ & $7,241 \mathrm{E}-07$ & $2,160 \mathrm{E}-03$ & $1,030 \mathrm{E}-05$ \\
\hline 25,5 & $-1777,212$ & 11,241 & $1,130 \mathrm{E}-03$ & $6,974 \mathrm{E}-07$ & $1,880 \mathrm{E}-03$ & $7,344 \mathrm{E}-06$ \\
\hline 20,5 & $-1865,019$ & 12,489 & $1,050 \mathrm{E}-03$ & $6,625 \mathrm{E}-07$ & $1,680 \mathrm{E}-03$ & $7,563 \mathrm{E}-06$ \\
\hline 15,5 & $-1881,320$ & 13,044 & $1,000 \mathrm{E}-03$ & $6,178 \mathrm{E}-07$ & $1,580 \mathrm{E}-03$ & $5,431 \mathrm{E}-06$ \\
\hline 10,5 & $-1964,846$ & 13,935 & $9,600 \mathrm{E}-04$ & $6,075 \mathrm{E}-07$ & $1,460 \mathrm{E}-03$ & $6,268 \mathrm{E}-06$ \\
\hline 5,5 & $-1995,601$ & 14,308 & $9,300 \mathrm{E}-04$ & $5,838 \mathrm{E}-07$ & $1,430 \mathrm{E}-03$ & $5,094 \mathrm{E}-06$ \\
\hline 0,0 & $-2013,649$ & 14,698 & $9,200 \mathrm{E}-04$ & $5,821 \mathrm{E}-07$ & $1,380 \mathrm{E}-03$ & $4,061 \mathrm{E}-06$ \\
\hline
\end{tabular}


Tabela 5.3: Potência Relativa, Reatividade Generalizada e Índice de Subcriticalidade em função da posição dos bancos de controle.

\begin{tabular}{|c|c|c|c|c|c|c|c|c|}
\hline $\begin{array}{c}\text { Posição } \\
\text { dos BCs (\%) }\end{array}$ & $\boldsymbol{P}_{N}$ & $\sigma_{P N}$ & $\begin{array}{c}\boldsymbol{\rho}_{\text {gen }} \\
(\mathrm{pcm})\end{array}$ & $\begin{array}{c}\boldsymbol{\sigma}_{\boldsymbol{\rho g} \boldsymbol{e n}} \\
(\mathrm{pcm})\end{array}$ & $\begin{array}{l}\boldsymbol{\Sigma} \boldsymbol{\rho}_{g e n} \\
(\mathrm{pcm})\end{array}$ & $\begin{array}{l}\sigma_{\Sigma \rho}^{(a)} \\
(\mathrm{pcm})\end{array}$ & $\begin{array}{c}\zeta \\
(\mathrm{pcm})\end{array}$ & $\begin{array}{c}\boldsymbol{\sigma}_{\boldsymbol{\zeta}} \\
(\mathrm{pcm})\end{array}$ \\
\hline 93,0 & & & & & & & 135,58 & 12,60 \\
\hline 90,5 & 0,599 & 0,026 & $-90,58$ & 22,68 & $-90,58$ & 22,68 & 196,98 & 15,09 \\
\hline 88,0 & 0,669 & 0,027 & $-97,36$ & 20,14 & $-187,94$ & 30,33 & 293,57 & 18,39 \\
\hline 85,5 & 0,732 & 0,027 & $-107,31$ & 19,03 & $-295,25$ & 35,81 & 359,01 & 20,38 \\
\hline 83,0 & 0,744 & 0,027 & $-123,26$ & 20,20 & $-418,51$ & 41,11 & 476,22 & 25,17 \\
\hline 80,5 & 0,770 & 0,030 & $-142,40$ & 23,85 & $-560,91$ & 47,53 & 627,59 & 33,24 \\
\hline 78,0 & 0,857 & 0,037 & $-104,42$ & 29,08 & $-665,32$ & 55,72 & 698,34 & 33,05 \\
\hline 75,5 & 0,803 & 0,031 & $-171,50$ & 30,64 & $-836,83$ & 63,59 & 847,94 & 34,94 \\
\hline 70,5 & 0,723 & 0,025 & $-325,15$ & 36,30 & $-1161,98$ & 73,22 & 1096,08 & 47,09 \\
\hline 65,5 & 0,730 & 0,029 & $-406,13$ & 51,56 & $-1568,10$ & 89,55 & 1362,16 & 62,19 \\
\hline 63,0 & 0,844 & 0,035 & $-251,34$ & 58,28 & $-1819,44$ & 106,84 & 1566,21 & 67,38 \\
\hline 60,5 & 0,859 & 0,034 & $-256,07$ & 65,05 & $-2075,51$ & 125,09 & 1624,86 & 65,95 \\
\hline 58,0 & 0,794 & 0,030 & $-422,28$ & 66,37 & $-2497,79$ & 141,61 & 2029,18 & 78,37 \\
\hline 55,5 & 0,919 & 0,033 & $-177,80$ & 74,86 & $-2675,60$ & 160,18 & 2224,21 & 96,79 \\
\hline 53,0 & 0,930 & 0,039 & $-168,32$ & 94,99 & $-2843,92$ & 186,22 & 2220,65 & 99,39 \\
\hline 50,5 & 0,884 & 0,038 & $-292,57$ & 98,78 & $-3136,48$ & 210,80 & 2684,64 & 80,79 \\
\hline 45,5 & 0,809 & 0,018 & $-633,12$ & 64,94 & $-3769,61$ & 220,58 & 3112,87 & 94,60 \\
\hline 43,0 & 0,869 & 0,020 & $-471,14$ & 75,33 & $-4240,75$ & 233,09 & 3481,29 & 97,98 \\
\hline 40,5 & 0,904 & 0,018 & $-370,43$ & 72,82 & $-4611,18$ & 244,20 & 3654,17 & 102,79 \\
\hline 35,5 & 0,853 & 0,018 & $-629,67$ & 79,82 & $-5240,85$ & 256,91 & 4257,04 & 119,75 \\
\hline 33,0 & 0,953 & 0,021 & $-212,08$ & 95,62 & $-5452,94$ & 274,13 & 4183,35 & 122,67 \\
\hline 30,5 & 0,889 & 0,020 & $-521,30$ & 98,24 & $-5974,24$ & 291,20 & 4638,31 & 132,33 \\
\hline 25,5 & 0,939 & 0,021 & $-302,32$ & 106,11 & $-6276,56$ & 309,93 & 4837,58 & 142,29 \\
\hline 20,5 & 0,926 & 0,022 & $-384,21$ & 118,65 & $-6660,76$ & 331,87 & 5015,71 & 148,62 \\
\hline 15,5 & 0,951 & 0,023 & $-258,28$ & 124,77 & $-6919,05$ & 354,55 & 5408,91 & 162,40 \\
\hline 10,5 & 0,976 & 0,025 & $-132,53$ & 137,61 & $-7051,57$ & 380,32 & 5168,75 & 157,28 \\
\hline 5,5 & 0,916 & 0,024 & $-471,17$ & 136,45 & $-7522,74$ & 404,05 & 5865,71 & 171,19 \\
\hline 0,0 & 1,029 & 0,025 & 168,01 & 143,36 & $-7354,73$ & 428,73 & & \\
\hline
\end{tabular}

${ }^{\text {(a) }} \boldsymbol{\sigma}_{\Sigma \boldsymbol{\rho}}=\sqrt{\sum\left(\sigma_{\rho g e n}\right)^{2}}$.

As incertezas de $\zeta, \rho_{g e n}$ e $P_{N}$ foram calculadas por meio do método padrão de propagação de erro das Eqs. (2.17), (2.18) e (2.20), respectivamente, como: 


$$
\begin{aligned}
& \sigma_{\rho_{g e n}}^{2}=\left[\left(B_{b} l_{e f f}+\beta_{e f f}\right) P_{N}\right]^{2}\left[2^{2}\left(\frac{\sigma_{R_{b}}^{2}}{R_{b}^{2}}+\frac{\sigma_{R_{a}}^{2}}{R_{a}^{2}}+\frac{\sigma_{B_{a}}^{2}}{B_{a}^{2}}\right)+\frac{\sigma_{C_{b}}^{2}+\sigma_{\Phi_{b}}^{2}}{\left(\Phi_{b}-C_{b}\right)^{2}}+\frac{\sigma_{C_{a}}^{2}+\sigma_{\Phi_{a}}^{2}}{\left(\Phi_{a}-C_{a}\right)^{2}}\right]+ \\
& +\sigma_{B_{b}}^{2}\left[l_{e f f}+P_{N} l_{e f f}+\frac{2 \beta_{e f f} P_{N}}{B_{b}}\right]^{2}+\left(1-P_{N}\right)^{2}\left(B_{b}^{2} \sigma_{l_{e f f}}^{2}+\sigma_{\beta_{e f f}}^{2}\right), \\
& \sigma_{\zeta}^{2}=\left[\left(B_{b} l_{e f f}+\beta_{e f f}\right) P_{N}\right]^{2}\left[2^{2}\left(\frac{\sigma_{R_{b}}^{2}}{R_{b}^{2}}+\frac{\sigma_{R_{a}}^{2}}{R_{a}^{2}}+\frac{\sigma_{B_{a}}^{2}}{B_{a}^{2}}\right)+\frac{\sigma_{C_{b}}^{2}+\sigma_{\Phi_{b}}^{2}}{\left(\Phi_{b}-C_{b}\right)^{2}}+\frac{\sigma_{C_{a}}^{2}+\sigma_{\Phi_{a}}^{2}}{\left(\Phi_{a}-C_{a}\right)^{2}}\right] \\
& +\sigma_{B_{b}}^{2}\left[P_{N} l_{e f f}+\frac{2 \beta_{e f f} P_{N}}{B_{b}}\right]^{2}+P_{N}^{2}\left(B_{b}^{2} \sigma_{l_{e f f}}^{2}+\sigma_{\beta_{e f f}}^{2}\right), \\
& \sigma_{P_{N}}=P_{N} \sqrt{2^{2}\left(\frac{\sigma_{R_{b}}^{2}}{R_{b}^{2}}+\frac{\sigma_{R_{a}}^{2}}{R_{a}^{2}}+\frac{\sigma_{B_{a}}^{2}}{B_{a}^{2}}+\frac{\sigma_{B_{b}}^{2}}{B_{b}^{2}}\right)+\frac{\sigma_{C_{b}}^{2}+\sigma_{\Phi_{b}}^{2}+\frac{\sigma_{C_{a}}^{2}+\sigma_{\Phi_{a}}^{2}}{\left(\Phi_{b}-C_{b}\right)^{2}}}{\left(\Phi_{a}-C_{a}\right)^{2}}}
\end{aligned}
$$

onde, os subíndices $a$ e $b$ têm o mesmo significado que anteriormente e assumiu-se que os parâmetros não estão correlacionados entre si.

As incertezas dos parâmetros nas Eqs. (5.1), (5.2) e (5.3) foram obtidas como:

- $\sigma_{B_{a}}, \sigma_{B_{b}}, \sigma_{C_{a}}$ e $\sigma_{C_{b}}$ : diretamente dos ajustes de mínimos quadrados das APSD. Essas são dadas pelos elementos diagonais da matriz covariância;

- $\sigma_{\beta_{e f f}}$ e $\sigma_{l_{e f f}}$ : da seção 3.6 de IPEN(MB01)-LWR-RESR-001(Dos Santos, et al., 2004a);

- $\sigma_{R_{a}}$ e $\sigma_{R_{b}}$ : como desvio padrão da média. O número de dados que contêm a contagem total é o mesmo do número da média da APSD $^{1}$;

- $\sigma_{\Phi_{a}}$ e $\sigma_{\Phi_{b}}$ : como desvio padrão da média as APSD na região do primeiro plateau $(\sim 2$ a $9 \mathrm{~Hz})$.

\footnotetext{
${ }^{1}$ As APSDs são obtidas com um número específico de médias, por exemplo, 900. Neste caso, a quantidade de valores de contagem total registrado, em um comprimento de registro, é, 900, também. Ou seja, 900 valores de contagem total são usados para obter um valor médio e o respectivo desvio padrão da média.
} 
As contribuições de cada um desses termos na incerteza da $\sigma_{\rho_{g e n}}^{2}$ na Eq. (5.1) são mostradas na Tabela 5.4. Como pode ser notado claramente, os principais contribuintes são $B_{b}, B_{a}, \Phi_{b}^{p}$ e $\Phi_{a}^{p}$. A influência do $l_{e f f}$ torna-se considerável somente nos estados próximos à criticalidade, mas desprezível à medida que a relatividade diminui. Na Tabela 5.5 são dados os principais contribuintes em $\sigma_{P_{N}}^{2}$, que novamente, são $B_{b}, B_{a}, \Phi_{b}^{p}$ e $\Phi_{a}^{p}$.

As sensibilidades dadas nas Tabelas $\mathbf{5 . 4}$ e $\mathbf{5 . 5}$ são importantes para experimentos futuros, em que o objetivo principal seja a redução de incertezas para obtenção de medidas da qualidade de benchmark. 
Tabela 5.4: Contribuições dos principais termos na determinação de $\sigma_{\rho_{g e n}}^{2}$ na Eq. (5.1).

\begin{tabular}{|c|c|c|c|c|c|c|}
\hline $\begin{array}{c}\text { Posição de } \\
\text { Bancos de Controle } \\
(\%)\end{array}$ & $\begin{array}{c}B_{b} \\
(\%)\end{array}$ & $\begin{array}{c}B_{a} \\
(\%)\end{array}$ & $\begin{array}{l}\Phi_{b}^{p} \\
(\%) \\
\end{array}$ & $\begin{array}{l}\Phi_{a}^{p} \\
(\%)\end{array}$ & $\begin{array}{l}l_{\text {eff }} \\
(\%)\end{array}$ & $\begin{array}{c}\text { Restantes } \\
(\%)\end{array}$ \\
\hline 93,0 & - & & - & - & - & - \\
\hline 90,5 & 28,4 & 5,2 & 11,1 & 9,1 & 42,8 & 3,4 \\
\hline 88,0 & 32,9 & 8,7 & 7,0 & 20,3 & 29,0 & 2,1 \\
\hline 85,5 & 35,3 & 15,1 & 20,2 & 11,4 & 16,9 & 1,1 \\
\hline 83,0 & 36,5 & 17,0 & 9,7 & 22,6 & 13,2 & 0,8 \\
\hline 80,5 & 34,4 & 17,5 & 30,6 & 9,6 & 7,4 & 0,4 \\
\hline 78,0 & 28,0 & 18,6 & 22,1 & 29,2 & 1,8 & 0,2 \\
\hline 75,5 & 34,2 & 24,0 & 11,7 & 25,8 & 4,0 & 0,2 \\
\hline 70,5 & 41,4 & 19,2 & 10,6 & 20,2 & 8,3 & 0,2 \\
\hline 65,5 & 43,5 & 18,2 & 25,1 & 8,1 & 4,9 & 0,2 \\
\hline 63,0 & 23,8 & 24,2 & 27,4 & 23,2 & 1,3 & 0,1 \\
\hline 60,5 & 31,4 & 19,4 & 19,1 & 28,9 & 1,0 & 0,1 \\
\hline 58,0 & 36,9 & 26,4 & 13,9 & 20,0 & 2,5 & 0,1 \\
\hline 55,5 & 37,1 & 32,0 & 13,7 & 16,7 & 0,3 & 0,1 \\
\hline 53,0 & 30,4 & 24,7 & 34,4 & 10,2 & 0,2 & 0,1 \\
\hline 50,5 & 29,2 & 25,5 & 12,9 & 31,8 & 0,5 & 0,1 \\
\hline 45,5 & 26,6 & 18,7 & 39,8 & 10,0 & 4,8 & 0,1 \\
\hline 43,0 & 25,4 & 20,0 & 13,3 & 39,2 & 1,9 & 0,2 \\
\hline 40,5 & 29,0 & 28,5 & 23,3 & 17,7 & 1,2 & 0,2 \\
\hline 35,5 & 30,2 & 23,6 & 21,6 & 21,5 & 2,8 & 0,2 \\
\hline 33,0 & 38,1 & 23,3 & 17,7 & 20,3 & 0,2 & 0,3 \\
\hline 30,5 & 26,9 & 33,0 & 22,3 & 16,3 & 1,2 & 0,3 \\
\hline 25,5 & 32,9 & 24,6 & 18,3 & 23,4 & 0,4 & 0,3 \\
\hline 20,5 & 32,6 & 26,6 & 24,0 & 16,0 & 0,5 & 0,4 \\
\hline 15,5 & 32,9 & 29,0 & 14,2 & 23,3 & 0,2 & 0,4 \\
\hline 10,5 & 32,0 & 29,7 & 24,3 & 13,5 & 0,0 & 0,4 \\
\hline 5,5 & 32,7 & 28,9 & 14,9 & 22,6 & 0,5 & 0,4 \\
\hline 0,0 & 34,5 & 34,4 & 13,0 & 17,4 & 0,1 & 0,5 \\
\hline
\end{tabular}


Tabela 5.5: Contribuições dos principais termos na determinação de $\sigma_{P_{N}}^{2}$.

\begin{tabular}{|c|c|c|c|c|c|}
\hline $\begin{array}{c}\text { Posição de } \\
\text { Bancos de } \\
\text { Controle (\%) }\end{array}$ & $B_{b}(\%)$ & $B_{a}(\%)$ & $\Phi_{b}^{p}(\%)$ & $\Phi_{a}^{p}(\%)$ & Restantes (\%) \\
\hline 93,0 & - & - & - & - & - \\
\hline 90,5 & 15,8 & 17,3 & 36,6 & 30,2 & 0,1 \\
\hline 88,0 & 20,5 & 19,2 & 15,4 & 44,6 & 0,1 \\
\hline 85,5 & 24,5 & 24,4 & 32,5 & 18,3 & 0,2 \\
\hline 83,0 & 26,3 & 25,3 & 14,5 & 33,7 & 0,2 \\
\hline 80,5 & 25,1 & 22,6 & 39,6 & 12,5 & 0,2 \\
\hline 78,0 & 22,7 & 20,5 & 24,4 & 32,3 & 0,2 \\
\hline 75,5 & 26,9 & 28,4 & 13,9 & 30,5 & 0,2 \\
\hline 70,5 & 32,4 & 26,0 & 14,3 & 27,2 & 0,1 \\
\hline 65,5 & 34,1 & 23,3 & 32,1 & 10,3 & 0,1 \\
\hline 63,0 & 19,8 & 25,9 & 29,4 & 24,9 & 0,1 \\
\hline 60,5 & 27,2 & 20,9 & 20,6 & 31,2 & 0,1 \\
\hline 58,0 & 30,6 & 30,3 & 16,0 & 23,0 & 0,1 \\
\hline 55,5 & 34,6 & 33,4 & 14,3 & 17,5 & 0,2 \\
\hline 53,0 & 28,4 & 25,4 & 35,5 & 10,5 & 0,2 \\
\hline 50,5 & 26,1 & 26,8 & 13,5 & 33,4 & 0,1 \\
\hline 45,5 & 22,9 & 21,0 & 44,7 & 11,2 & 0,2 \\
\hline 43,0 & 22,7 & 21,3 & 14,1 & 41,7 & 0,2 \\
\hline 40,5 & 26,9 & 29,9 & 24,5 & 18,6 & 0,2 \\
\hline 35,5 & 27,1 & 25,7 & 23,5 & 23,4 & 0,3 \\
\hline 33,0 & 36,8 & 23,9 & 18,2 & 20,8 & 0,3 \\
\hline 30,5 & 24,6 & 34,6 & 23,4 & 17,1 & 0,3 \\
\hline 25,5 & 31,4 & 25,3 & 18,8 & 24,1 & 0,3 \\
\hline 20,5 & 30,8 & 27,5 & 24,8 & 16,5 & 0,4 \\
\hline 15,5 & 31,7 & 29,6 & 14,5 & 23,8 & 0,4 \\
\hline 10,5 & 31,4 & 30,0 & 24,5 & 13,7 & 0,5 \\
\hline 5,5 & 30,6 & 30,0 & 15,5 & 23,4 & 0,5 \\
\hline 0,0 & 35,3 & 34,1 & 12,9 & 17,2 & 0,5 \\
\hline
\end{tabular}

Além do mais, é razoável assumir que, na posição de 93\% dos BCs retirados, ou seja, no estado crítico, a reatividade generalizada, $\rho_{g e n}$, seja muito próxima à reatividade determinada pela cinética pontual clássica, i.e., pela equação da constante de decaimento de nêutrons prontos, $B$ : 


$$
B=\frac{\left(\rho-\beta_{e f f}\right)}{l_{e f f}}
$$

onde $B$ é extraído a partir dos dados experimentais medidos e apresentados na Tabela 5.2; $\beta_{\text {eff }}$ e $l_{\text {eff }}$ são os mesmos que foram usados anteriormente; e as incertezas, determinadas pelo método de propagação de erros.

Assumiu-se que a reatividade na posição de $93 \%$ tem o valor de -144.33 $( \pm 18.82)$ pcm. Além do mais, através do procedimento de medidas que segue uma abordagem experimental aplicada para determinação de reatividades para todas as configurações críticas de IPEN/MB-01 ICSBEP, foi medida a reatividade de $10( \pm 2.97)$ pcm para o caso em que os BCs foram totalmente retirados. Este valor foi medido por um reatímetro do modelo de cinética inversa. Os parâmetros cinéticos efetivos usados nessas medidas são dados na Tabela 5.6 e foram obtidos a partir de IPEN(MB-01)-LWR-RESR-001 (Dos Santos, 2009).

Tabela 5.6: Parâmetros efetivos de nêutrons atrasados do reator IPEN/MB-01 utilizados para o caso dos bancos de controle totalmente retirados.

\begin{tabular}{|cc|}
\hline$\beta_{i}$ & $\lambda_{i}\left(s^{-1}\right)$ \\
\hline$(2.679 \pm 0.023) \mathrm{E}-4$ & $0.012456($ fixo $)$ \\
$(1.463 \pm 0.069) \mathrm{E}-3$ & $0.0319 \pm 0,0032$ \\
$(1.34 \pm 0.13) \mathrm{E}-3$ & $0.1085 \pm 0,0054$ \\
$(3.10 \pm 0.10) \mathrm{E}-3$ & $0.3054 \pm 0,0055$ \\
$(8.31 \pm 0.62) \mathrm{E}-4$ & $1.085 \pm 0,044$ \\
$(4.99 \pm 0.27) \mathrm{E}-4$ & $3.14 \pm 0,11$ \\
\hline \hline$\beta_{\text {eff }}=(7.50 \pm 0.19) \mathrm{E}-3$ & e $l_{e f f}=31.96( \pm 1.06) \mu \mathrm{s}$ \\
\hline
\end{tabular}


Em seguida, as reatividades, $\Sigma \rho_{\text {gen }}$, foram recalculadas incluindo as $\rho_{\text {gen }}$ das posições de $93 \%$ e de $100 \%$ dos BCs retirados. As incertezas das $\Sigma \rho_{\text {gen }}$ foram, também, recalculadas levando em consideração as incertezas desses novos dados pelo método padrão de propagação de erros. A Tabela 5.7 mostra os resultados desses cálculos e os valores da reatividade obtidos pela Eq. (5.4).

Tabela 5.7: Reatividade generalizada recalculadas, o índice de subcriticalidade com sinal invertido e a reatividade determinada pela cinética pontual clássica.

\begin{tabular}{|c|c|c|c|c|c|c|c|c|}
\hline $\begin{array}{c}\text { Posição } \\
\text { dos BCs } \\
(\%)\end{array}$ & $\begin{array}{c}\boldsymbol{\rho}_{\boldsymbol{g e n}} \\
(\mathrm{pcm})\end{array}$ & $\begin{array}{c}\boldsymbol{\sigma}_{\boldsymbol{\rho g e n}} \\
(\mathrm{pcm})\end{array}$ & $\begin{array}{l}\boldsymbol{\Sigma} \boldsymbol{\rho}_{\text {gen }} \\
(\mathrm{pcm})\end{array}$ & $\begin{array}{l}\boldsymbol{\sigma}_{\Sigma \boldsymbol{\rho} \operatorname{gen}} \\
(\mathrm{pcm})\end{array}$ & $-\zeta(\mathrm{pcm})$ & $\sigma_{\zeta}(\mathrm{pcm})$ & $\begin{array}{l}\boldsymbol{\rho}_{c p c}{ }^{(\mathbf{a})} \\
(\mathrm{pcm})\end{array}$ & $\begin{array}{c}\boldsymbol{\sigma}_{\boldsymbol{\rho c p c}} \\
(\mathrm{pcm})\end{array}$ \\
\hline 100,0 & 10,00 & 2,97 & 10,00 & 2,97 & & & & \\
\hline 93,0 & $-144,33$ & 18,82 & $-134,33$ & 19,05 & $-135,58$ & 12,60 & $-144,33$ & 18,82 \\
\hline 90,5 & $-90,58$ & 10,83 & $-224,91$ & 21,91 & $-196,98$ & 15,09 & $-226,16$ & 20,25 \\
\hline 88,0 & $-97,36$ & 11,62 & $-322,27$ & 24,80 & $-293,57$ & 18,39 & $-294,34$ & 21,71 \\
\hline 85,5 & $-107,31$ & 13,59 & $-429,58$ & 28,28 & $-359,01$ & 20,38 & $-400,88$ & 23,82 \\
\hline 83,0 & $-123,26$ & 15,69 & $-552,84$ & 32,34 & $-476,22$ & 25,17 & $-482,27$ & 25,52 \\
\hline 80,5 & $-142,40$ & 20,92 & $-695,24$ & 38,52 & $-627,59$ & 33,24 & $-618,62$ & 28,55 \\
\hline 78,0 & $-104,42$ & 28,20 & $-799,66$ & 47,74 & $-698,34$ & 33,05 & $-732,01$ & 31,20 \\
\hline 75,5 & $-171,50$ & 29,03 & $-971,16$ & 55,87 & $-847,94$ & 34,94 & $-869,84$ & 33,81 \\
\hline 70,5 & $-325,15$ & 33,58 & $-1296,31$ & 65,19 & $-1096,08$ & 47,09 & $-1173,09$ & 39,83 \\
\hline 65,5 & $-406,13$ & 49,77 & $-1702,44$ & 82,01 & $-1362,16$ & 62,19 & $-1502,21$ & 48,84 \\
\hline 63,0 & $-251,34$ & 57,77 & $-1953,77$ & 100,32 & $-1566,21$ & 67,38 & $-1613,50$ & 48,17 \\
\hline 60,5 & $-256,07$ & 64,68 & $-2209,84$ & 119,36 & $-1624,86$ & 65,95 & $-1822,28$ & 53,93 \\
\hline 58,0 & $-422,28$ & 65,58 & $-2632,13$ & 136,19 & $-2029,18$ & 78,37 & $-2047,14$ & 58,66 \\
\hline 55,5 & $-177,80$ & 74,75 & $-2809,93$ & 155,35 & $-2224,21$ & 96,79 & $-2206,98$ & 62,26 \\
\hline 53,0 & $-168,32$ & 94,92 & $-2978,25$ & 182,06 & $-2220,65$ & 99,39 & $-2392,53$ & 67,13 \\
\hline 50,5 & $-292,57$ & 98,61 & $-3270,82$ & 207,04 & $-2684,64$ & 80,79 & $-2513,21$ & 69,43 \\
\hline 45,5 & $-633,12$ & 64,25 & $-3903,94$ & 216,78 & $-3112,87$ & 94,60 & $-3317,76$ & 77,04 \\
\hline 43,0 & $-471,14$ & 75,05 & $-4375,08$ & 229,41 & $-3481,29$ & 97,98 & $-3584,01$ & 82,29 \\
\hline 40,5 & $-370,43$ & 72,67 & $-4745,52$ & 240,64 & $-3654,17$ & 102,79 & $-3851,72$ & 86,97 \\
\hline 35,5 & $-629,67$ & 79,48 & $-5375,19$ & 253,43 & $-4257,04$ & 119,75 & $-4283,84$ & 95,31 \\
\hline 33,0 & $-212,08$ & 95,59 & $-5587,27$ & 270,86 & $-4183,35$ & 122,67 & $-4469,12$ & 100,98 \\
\hline 30,5 & $-521,30$ & 98,09 & $-6108,57$ & 288,07 & $-4638,31$ & 132,33 & $-4704,65$ & 103,65 \\
\hline 25,5 & $-302,32$ & 106,07 & $-6410,89$ & 306,98 & $-4837,58$ & 142,29 & $-4940,63$ & 109,30 \\
\hline 20,5 & $-384,21$ & 118,60 & $-6795,10$ & 329,09 & $-5015,71$ & 148,62 & $-5221,79$ & 115,43 \\
\hline 15,5 & $-258,28$ & 124,75 & $-7053,38$ & 351,94 & $-5408,91$ & 162,40 & $-5273,99$ & 116,94 \\
\hline 10,5 & $-132,53$ & 137,60 & $-7185,91$ & 377,88 & $-5168,75$ & 157,28 & $-5541,44$ & 122,49 \\
\hline 5,5 & $-471,17$ & 136,39 & $-7657,08$ & 401,74 & $-5865,71$ & 171,19 & $-5639,92$ & 124,58 \\
\hline 0,0 & 168,01 & 143,35 & $-7489,07$ & 426,55 & & & $-5697,71$ & 126,02 \\
\hline
\end{tabular}

(a) Reatividade da Cinética Pontual Clássica 
Os resultados experimentais obtidos através do método proposto são mostrados na Fig. 5.1. Esta figura mostra a reatividade total obtida pela soma das $\rho_{g e n}$ parciais, o índice de subcriticalidade, tomado como negativo para fins comparativos, i.e., $-\zeta$, e as reatividades obtidas a partir da teoria cinética pontual clássica, $\rho_{c p c}$.

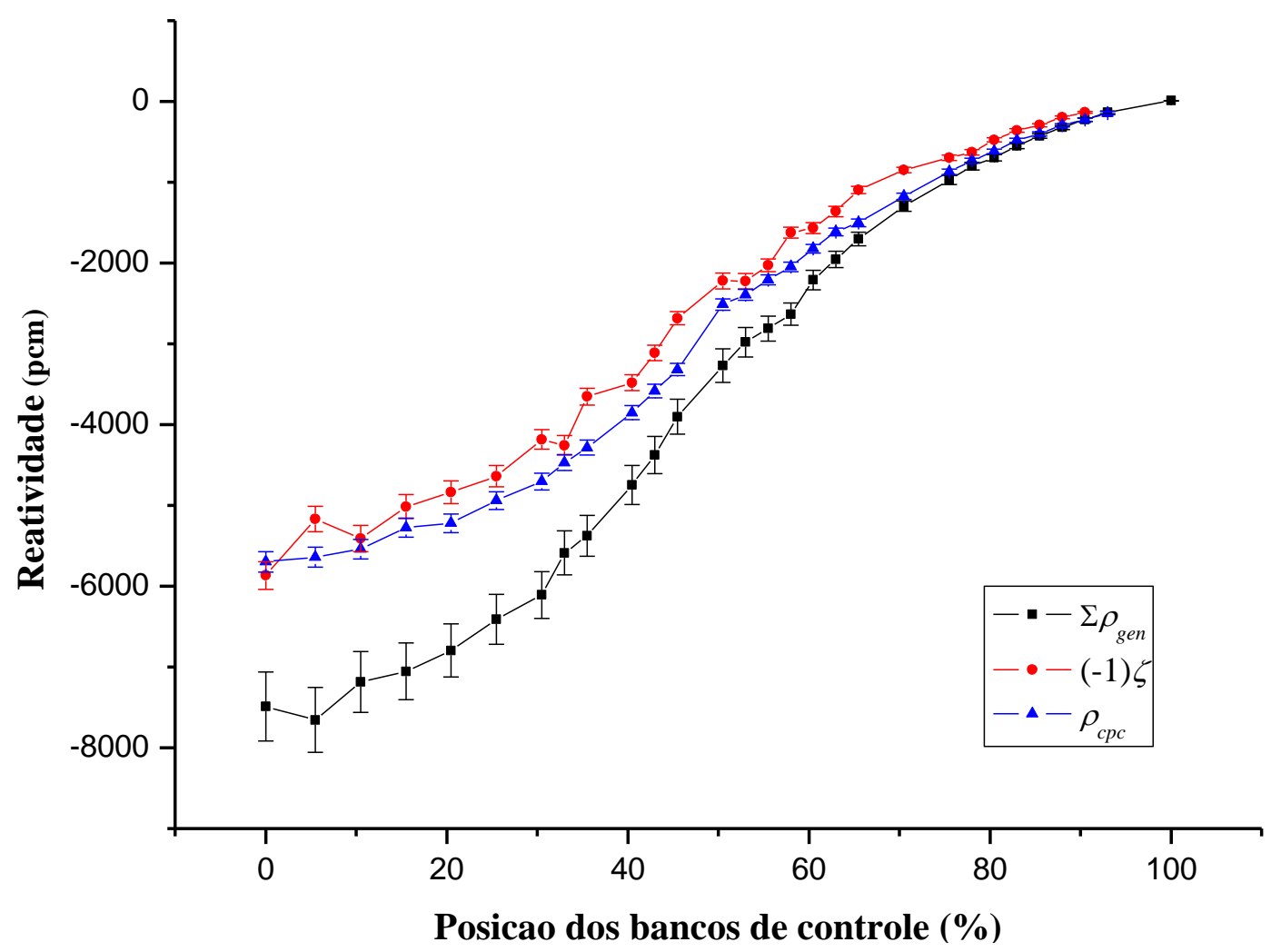

Fig. 5.1. Resultados experimentais para a reatividade subcrítica total $\left(\Sigma \rho_{g e n}\right), \zeta$ negativo e a reatividade subcrítica da cinética pontual clássica, $\rho_{c p c}$.

No sentido genérico, as grandezas têm o comportamento esperado, assemelhando-se a uma forma típica de um "S". No entanto, pode-se observar que as incertezas associadas a $\Sigma \rho_{\text {gen }}$ aumentam à medida que o sistema se torna cada vez mais subcrítico. Boa parte destas dificuldades surge da Eq. (2.17) que contém explicitamente o termo $\left(1-P_{N}\right)$ na expressão. Este termo impõe que, a potência relativa, $P_{N}$, seja obtida com precisão elevada e que os valores sejam suficientemente 
diferentes de 1. Essas condições são facilmente satisfeitas com os bancos controle perto da posição crítica. No entanto, à medida que os bancos de controle são inseridos, as estatísticas de contagem no detector e a resolução da APSD tornam-se cada vez piores, e o valor da potência relativa, $P_{N}$, aproxima-se de 1.0.

Por outro lado, porém, as inserções do banco de controle tem que induzir mudança na seção de choque macroscópica suficientemente pequena para que a condição para o método de perturbação de primeira ordem permaneça válida. Estas dificuldades foram parcialmente superadas com a utilização de detectores mais sensíveis e com a introdução de uma fonte externa de Am-Be no sistema.

Um aspecto notável dessas medidas é que a teoria da cinética pontual clássica não descreve as reatividades medidas. Em vez disso, as reatividades deste modelo em função da posição do banco de controle ficam próximas do índice de subcriticalidade (६) para uma determinada configuração de fonte do experimento. (De fato, mantendo a condição assumida para este trabalho na qual a relação entre o espectro de nêutrons prontos e o espectro de nêutrons atrasados, $\alpha_{p d}$, é $1, \zeta$ se aproxima do $\rho_{C P C} \mid$ quando $P_{N}$ tende a unidade.)

Dentre os parâmetros que podem ser deduzidos a partir dos resultados da Tabela 5.7 estão: o fator de multiplicação efetivo, $k_{\text {effj; }}$ o fator de multiplicação subcrítica, $k_{\text {sub }}$; e a razão do módulo da reatividade total para o índice de subcriticalidade correspondente ( $\zeta), \varphi^{*}$,que são dados por:

$$
\begin{aligned}
& k_{e f f}=\frac{1}{\left(1-\Sigma \rho_{\text {gen }}\right)} ; \\
& k_{\text {sub }}=\frac{1}{(1+\zeta)} ;
\end{aligned}
$$




$$
\varphi^{*}=\left(\frac{1}{k_{e f f}}-1\right) /\left(\frac{1}{k_{\text {sub }}}-1\right) \text {. }
$$

E as respectivas incertezas são dadas como:

$$
\begin{aligned}
& \sigma_{k_{e f f}}=\left(\frac{1}{1-\Sigma \rho_{g e n}}\right)^{2} \cdot \sigma_{\Sigma \rho_{g e n}} ; \\
& \sigma_{k_{\text {sub }}}=\left(\frac{1}{1+\zeta}\right)^{2} \cdot \sigma_{\zeta} ; \\
& \sigma_{\varphi^{*}}=\sqrt{\left(\frac{1}{\zeta}\right)^{2} \cdot \sigma_{\Sigma \rho}^{2}+\left(\frac{\Sigma \rho}{\zeta^{2}}\right)^{2} \cdot \sigma_{\zeta}^{2}} .
\end{aligned}
$$

Os valores desses três parâmetros e suas respectivas incertezas são mostrados na Tabela 5.8. 
Tabela 5.8: Parâmetros derivados a partir dos dados medidos

\begin{tabular}{|c|c|c|c|c|c|c|}
\hline $\begin{array}{c}\text { Posição } \\
\operatorname{dos} \operatorname{BCs}(\%)\end{array}$ & $k_{e f f}$ & $\sigma_{k_{e f f}}$ & $k_{s u b}$ & $\sigma_{k_{s u b}}$ & $\varphi^{*}$ & $\sigma_{\varphi^{*}}$ \\
\hline 100,0 & 1,00010 & 0,00003 & & & & \\
\hline 93,0 & 0,99866 & 0,00019 & 0,99865 & 0,00013 & 0,99 & 0,13 \\
\hline 90,5 & 0,99776 & 0,00022 & 0,99803 & 0,00015 & 1,14 & 0,12 \\
\hline 88,0 & 0,99679 & 0,00025 & 0,99707 & 0,00018 & 1,10 & 0,09 \\
\hline 85,5 & 0,99572 & 0,00028 & 0,99642 & 0,00020 & 1,20 & 0,09 \\
\hline 83,0 & 0,99450 & 0,00032 & 0,99526 & 0,00025 & 1,16 & 0,08 \\
\hline 80,5 & 0,99310 & 0,00038 & 0,99376 & 0,00033 & 1,11 & 0,08 \\
\hline 78,0 & 0,99207 & 0,00047 & 0,99307 & 0,00033 & 1,15 & 0,07 \\
\hline 75,5 & 0,99038 & 0,00055 & 0,99159 & 0,00034 & 1,15 & 0,06 \\
\hline 70,5 & 0,98720 & 0,00064 & 0,98916 & 0,00046 & 1,18 & 0,07 \\
\hline 65,5 & 0,98326 & 0,00079 & 0,98656 & 0,00061 & 1,25 & 0,07 \\
\hline 63,0 & 0,98084 & 0,00097 & 0,98458 & 0,00065 & 1,25 & 0,07 \\
\hline 60,5 & 0,97838 & 0,00114 & 0,98401 & 0,00064 & 1,36 & 0,07 \\
\hline 58,0 & 0,97435 & 0,00129 & 0,98011 & 0,00075 & 1,30 & 0,06 \\
\hline 55,5 & 0,97267 & 0,00147 & 0,97824 & 0,00093 & 1,26 & 0,07 \\
\hline 53,0 & 0,97108 & 0,00172 & 0,97828 & 0,00095 & 1,34 & 0,07 \\
\hline 50,5 & 0,96833 & 0,00194 & 0,97386 & 0,00077 & 1,22 & 0,05 \\
\hline 45,5 & 0,96243 & 0,00201 & 0,96981 & 0,00089 & 1,25 & 0,05 \\
\hline 43,0 & 0,95808 & 0,00211 & 0,96636 & 0,00092 & 1,26 & 0,05 \\
\hline 40,5 & 0,95469 & 0,00219 & 0,96475 & 0,00096 & 1,30 & 0,05 \\
\hline 35,5 & 0,94899 & 0,00228 & 0,95917 & 0,00110 & 1,26 & 0,05 \\
\hline 33,0 & 0,94708 & 0,00243 & 0,95985 & 0,00113 & 1,34 & 0,05 \\
\hline 30,5 & 0,94243 & 0,00256 & 0,95567 & 0,00121 & 1,32 & 0,05 \\
\hline 25,5 & 0,93975 & 0,00271 & 0,95386 & 0,00129 & 1,33 & 0,05 \\
\hline 20,5 & 0,93637 & 0,00289 & 0,95224 & 0,00135 & 1,35 & 0,05 \\
\hline 15,5 & 0,93411 & 0,00307 & 0,94869 & 0,00146 & 1,30 & 0,05 \\
\hline 10,5 & 0,93296 & 0,00329 & 0,95085 & 0,00142 & 1,39 & 0,05 \\
\hline 5,5 & 0,92888 & 0,00347 & 0,94459 & 0,00153 & 1,31 & 0,05 \\
\hline 0,0 & 0,93033 & 0,00369 & & & & \\
\hline
\end{tabular}

\subsection{Os resultados da CPSD}

Como pode ser notado na Fig. 4.5, houve necessidade de restringir o intervalo do ajuste dos mínimos quadrados. Em geral, a frequência máxima a ser considerada 
no ajuste ficou entre $B / 2 \pi$ e $B / \pi$. Os primeiros três pontos da CPSD, de 0 a $2 \mathrm{~Hz}$, foram desconsiderados por serem atípicos.

As medidas foram limitadas até a posição de 40.5\%, devido à limitação da eficiência do detector ${ }^{3} \mathrm{He}$ de sensibilidade média. Os resultados obtidos nos níveis mais subcríticos do que aquele a $40.5 \%$ apresentaram flutuações consideráveis e, conseqüentemente, foram ignorados.

Os valores de $B, \Phi^{p}$ e as contagens dos dois detectores, $R_{1}$ e $R_{2}$ são dados na Tabela 5.9.

Tabela 5.9. Contagens nos detectores 1 e 2 , o parâmetro $B$ e o plateau superior da CPSD.

\begin{tabular}{|c|c|c|c|c|c|c|c|c|}
\hline $\begin{array}{c}\text { Posição } \\
\text { dos } \\
\text { BCs } \\
(\%)\end{array}$ & $\mathbf{R}_{1}$ & $\sigma_{\mathrm{R} 1}$ & $\mathbf{R}_{2}$ & $\sigma_{\mathrm{R} 2}$ & $\mathbf{B}\left(\mathrm{s}^{-1}\right)$ & $\boldsymbol{\sigma}_{\mathbf{B}}\left(\mathrm{s}^{-1}\right)^{(\mathbf{a})}$ & $\Phi^{\mathbf{p}(\mathbf{b})}$ & $\boldsymbol{\sigma}_{\Phi \mathbf{p}}{ }^{(\mathbf{b}),(\mathbf{c})}$ \\
\hline 93,0 & 10652,49 & 7,55 & 10906,61 & 7,80 & $-271,829$ & 2,411 & $8,68 \mathrm{E}-04$ & $1,76 \mathrm{E}-06$ \\
\hline 90,5 & 6899,25 & 5,16 & 7084,60 & 5,51 & $-302,901$ & 2,831 & 4,83E-04 & $1,23 \mathrm{E}-06$ \\
\hline 88,0 & 4908,76 & 4,18 & 4908,76 & 4,18 & $-325,836$ & 3,208 & $3,02 \mathrm{E}-04$ & $5,15 \mathrm{E}-07$ \\
\hline 85,5 & 3677,58 & 3,49 & 3816,39 & 3,55 & $-356,343$ & 3,645 & $1,95 \mathrm{E}-04$ & $3,89 \mathrm{E}-07$ \\
\hline 83,0 & 2870,12 & 2,79 & 2991,89 & 3,00 & $-392,000$ & 4,396 & $1,26 \mathrm{E}-04$ & 1,79E-07 \\
\hline 80,5 & 2311,55 & 2,22 & 2420,65 & 2,38 & $-431,095$ & 5,449 & $8,34 \mathrm{E}-05$ & $1,49 \mathrm{E}-07$ \\
\hline 78,0 & 1899,57 & 2,01 & 2004,68 & 2,11 & $-470,928$ & 6,482 & $5,79 \mathrm{E}-05$ & 7,03E-08 \\
\hline 75,5 & 1597,41 & 1,75 & 1694,22 & 1,87 & $-510,971$ & 7,743 & $4,02 \mathrm{E}-05$ & $6,19 \mathrm{E}-08$ \\
\hline 73,0 & 1363,21 & 1,51 & 1454,34 & 1,64 & $-562,312$ & 10,833 & $2,87 \mathrm{E}-05$ & 2,93E-08 \\
\hline 70,5 & 9080,15 & 6,50 & 3693,64 & 3,04 & $-611,830$ & 4,169 & $4,26 \mathrm{E}-04$ & $5,77 \mathrm{E}-07$ \\
\hline 68,0 & 8028,29 & 5,90 & 3294,63 & 2,63 & $-667,311$ & 4,947 & $3,20 \mathrm{E}-04$ & $3,09 \mathrm{E}-07$ \\
\hline 65,5 & 7039,01 & 5,24 & 2924,16 & 2,61 & $-731,369$ & 6,124 & 2,34E-04 & $2,29 \mathrm{E}-07$ \\
\hline 63,0 & 47230,60 & 11,01 & 21287,88 & 6,39 & $-796,389$ & 6,540 & $1,17 \mathrm{E}-03$ & $9,01 \mathrm{E}-07$ \\
\hline 60,5 & 42283,97 & 9,50 & 18975,25 & 5,71 & $-866,188$ & 7,989 & $9,24 \mathrm{E}-04$ & 6,33E-07 \\
\hline 58,0 & 37913,38 & 9,18 & 16991,61 & 5,29 & $-938,785$ & 9,262 & 7,33E-04 & $7,45 \mathrm{E}-07$ \\
\hline 55,5 & 34052,33 & 8,21 & 15268,07 & 4,78 & $-1008,850$ & 10,783 & $5,87 \mathrm{E}-04$ & $5,91 \mathrm{E}-07$ \\
\hline 53,0 & 30677,65 & 7,71 & 13789,13 & 4,44 & $-1058,931$ & 12,388 & 4,76E-04 & $3,81 \mathrm{E}-07$ \\
\hline 50,5 & 27718,30 & 7,06 & 12490,55 & 4,34 & $-1131,489$ & 14,225 & $3,89 \mathrm{E}-04$ & $3,56 \mathrm{E}-07$ \\
\hline 48,0 & 25124,80 & 6,62 & 11360,34 & 4,08 & $-1229,849$ & 16,433 & $3,05 \mathrm{E}-04$ & $1,73 \mathrm{E}-07$ \\
\hline 45,5 & 22858,68 & 6,06 & 10377,12 & 3,97 & $-1291,414$ & 19,658 & $2,50 \mathrm{E}-04$ & $1,82 \mathrm{E}-07$ \\
\hline 43,0 & 20871,96 & 5,95 & 9514,59 & 3,56 & $-1371,973$ & 23,167 & $2,07 \mathrm{E}-04$ & $1,15 \mathrm{E}-07$ \\
\hline 40,5 & 18985,24 & 5,36 & 8700,72 & 3,41 & $-1384,543$ & 25,276 & $1,72 \mathrm{E}-04$ & $9,36 \mathrm{E}-08$ \\
\hline
\end{tabular}


Os valores de $P_{N}, \rho_{g e n}, \Sigma \rho_{g e n}$ e $\zeta$ calculados a partir dos dados da Tabela 5.9. são mostrados na Tabela 5.10.

Tabela 5.10. Potência relativa, reatividade parcial e total, e o índice de subcriticalidade obtidos por CPSD.

\begin{tabular}{|c|c|c|c|c|c|c|c|c|}
\hline $\begin{array}{c}\text { Posição } \\
\text { dos } \\
\text { BCs } \\
(\%)\end{array}$ & $\boldsymbol{P}_{N}$ & $\sigma_{P N}$ & $\begin{array}{c}\boldsymbol{\rho}_{\boldsymbol{g e n}} \\
(\mathrm{pcm})\end{array}$ & $\begin{array}{c}\boldsymbol{\sigma}_{\boldsymbol{\rho g} \boldsymbol{\rho} \boldsymbol{n}} \\
(\mathrm{pcm})\end{array}$ & $\begin{array}{c}\boldsymbol{\Sigma} \boldsymbol{\rho}_{\boldsymbol{g e n}} \\
(\mathrm{pcm})\end{array}$ & $\begin{array}{c}\boldsymbol{\sigma}_{\boldsymbol{\Sigma} \boldsymbol{\rho}} \\
(\mathrm{pcm})\end{array}$ & $\begin{array}{c}\boldsymbol{\zeta} \\
(\mathrm{pcm})\end{array}$ & $\begin{array}{c}\boldsymbol{\sigma}_{\zeta} \\
(\mathrm{pcm})\end{array}$ \\
\hline 93,0 & & & & & & & 133,84 & 11,77 \\
\hline 90,5 & 0,609 & 0,016 & $-86,04$ & 9,05 & $-86,04$ & 9,05 & 200,10 & 14,20 \\
\hline 88,0 & 0,682 & 0,019 & $-93,23$ & 9,97 & $-179,27$ & 13,46 & 294,53 & 17,28 \\
\hline 85,5 & 0,753 & 0,022 & $-96,48$ & 11,72 & $-275,75$ & 17,85 & 394,81 & 20,06 \\
\hline 83,0 & 0,782 & 0,024 & $-110,37$ & 13,93 & $-386,13$ & 22,64 & 513,69 & 23,84 \\
\hline 80,5 & 0,815 & 0,028 & $-116,67$ & 17,51 & $-502,80$ & 28,63 & 623,22 & 27,80 \\
\hline 78,0 & 0,822 & 0,031 & $-134,69$ & 21,27 & $-637,49$ & 35,66 & 769,29 & 33,73 \\
\hline 75,5 & 0,868 & 0,036 & $-116,84$ & 27,21 & $-754,33$ & 44,86 & 889,58 & 39,22 \\
\hline 73,0 & 0,847 & 0,042 & $-160,94$ & 33,56 & $-915,27$ & 56,02 & 1045,20 & 34,01 \\
\hline 70,5 & 0,864 & 0,016 & $-163,88$ & 16,20 & $-1079,15$ & 58,32 & 1222,94 & 38,57 \\
\hline 68,0 & 0,882 & 0,018 & $-163,79$ & 19,23 & $-1242,95$ & 61,40 & 1407,54 & 43,70 \\
\hline 65,5 & 0,884 & 0,020 & $-184,31$ & 23,38 & $-1427,25$ & 65,71 & 1461,08 & 44,67 \\
\hline 63,0 & 0,812 & 0,018 & $-338,95$ & 25,25 & $-1766,21$ & 70,39 & 1729,26 & 52,66 \\
\hline 60,5 & 0,855 & 0,021 & $-294,27$ & 30,50 & $-2060,48$ & 76,71 & 1943,00 & 60,60 \\
\hline 58,0 & 0,861 & 0,023 & $-312,99$ & 37,73 & $-2373,47$ & 85,49 & 2164,29 & 68,71 \\
\hline 55,5 & 0,873 & 0,025 & $-316,05$ & 44,53 & $-2689,52$ & 96,39 & 2405,93 & 78,83 \\
\hline 53,0 & 0,911 & 0,029 & $-234,77$ & 53,31 & $-2924,30$ & 110,15 & 2521,95 & 85,91 \\
\hline 50,5 & 0,878 & 0,030 & $-351,08$ & 60,65 & $-3275,37$ & 125,74 & 2837,90 & 99,98 \\
\hline 48,0 & 0,890 & 0,033 & $-350,07$ & 72,88 & $-3625,44$ & 145,34 & 3107,24 & 114,07 \\
\hline 45,5 & 0,918 & 0,037 & $-277,87$ & 84,82 & $-3903,32$ & 168,28 & 3261,01 & 129,90 \\
\hline 43,0 & 0,895 & 0,041 & $-382,04$ & 100,80 & $-4285,36$ & 196,16 & 3633,11 & 155,42 \\
\hline 40,5 & 0,986 & 0,049 & $-50,20$ & 125,46 & $-4335,56$ & 232,85 & 3586,84 & 163,72 \\
\hline
\end{tabular}

As incertezas foram calculadas pelo método padrão de propagação de erros e são dadas por:

$$
\sigma_{P_{N}}^{2}=P_{N}^{2}\left\{\left(\frac{\sigma_{R_{1, b}}}{R_{1, b}}\right)^{2}+\left(\frac{\sigma_{R_{1, a}}}{R_{1, a}}\right)^{2}+\left(\frac{\sigma_{R_{2, b}}}{R_{2, b}}\right)^{2}+\left(\frac{\sigma_{R_{2, a}}}{R_{2, a}}\right)^{2}+\left(\frac{\sigma_{\Phi_{a}^{p}}}{\Phi_{a}^{p}}\right)^{2}+\left(\frac{\sigma_{\Phi_{b}^{p}}}{\Phi_{b}^{p}}\right)^{2}+\right.
$$




$$
\begin{aligned}
& \left.+4\left[\left(\frac{\sigma_{B_{a}}}{B_{a}}\right)^{2}+\left(\frac{\sigma_{B_{b}}}{B_{b}}\right)^{2}\right]\right\} \\
& \sigma_{\rho_{g e n}}^{2}=\left(1-P_{N}\right)^{2}\left(B_{b}^{2} \sigma_{l_{e f f}}^{2}+\sigma_{\beta_{e f f}}^{2}\right)+\left(l_{e f f}-l_{e f f} P_{N}+\frac{2 \beta_{e f f} P_{N}}{B_{b}}\right)^{2} \sigma_{B_{b}}^{2}+ \\
& +\left(B_{b} l_{\text {eff }}+\beta_{\text {eff }}\right)^{2} P_{N}^{2}\left[\left(\frac{\sigma_{R_{1, b}}}{R_{1, b}}\right)^{2}+\left(\frac{\sigma_{R_{1, a}}}{R_{1, a}}\right)^{2}+\left(\frac{\sigma_{R_{2, b}}}{R_{2, b}}\right)^{2}+\left(\frac{\sigma_{R_{2, a}}}{R_{2, a}}\right)^{2}+\right. \\
& \left.+\left(\frac{\sigma_{\Phi_{a}^{p}}}{\Phi_{a}^{p}}\right)^{2}+\left(\frac{\sigma_{\Phi_{b}^{p}}}{\Phi_{b}^{p}}\right)^{2}+4\left(\frac{\sigma_{B_{b}}}{B_{b}}\right)^{2}\right] \\
& \sigma_{\zeta}^{2}=\left(l_{\text {eff }}+\frac{2 \beta_{\text {eff }}}{B_{b}}\right) P_{N}^{2} \sigma_{B_{b}}^{2}+\left(B_{b}^{2} \sigma_{l_{e f f}}^{2}+\sigma_{\beta_{e f f}}^{2}\right) P_{N}^{2}+\left(B_{b} l_{e f f}+\beta_{\text {eff }}\right)^{2} P_{N}^{2}\left[\left(\frac{\sigma_{R_{1, b}}}{R_{1, b}}\right)^{2}+\left(\frac{\sigma_{R_{1, a}}}{R_{1, a}}\right)^{2}+\right. \\
& \left.+\left(\frac{\sigma_{R_{2, b}}}{R_{2, b}}\right)^{2}+\left(\frac{\sigma_{R_{2, a}}}{R_{2, a}}\right)^{2}+\left(\frac{\sigma_{\Phi_{a}^{p}}}{\Phi_{a}^{p}}\right)^{2}+\left(\frac{\sigma_{\Phi_{b}^{p}}}{\Phi_{b}^{p}}\right)^{2}+4\left(\frac{\sigma_{B_{b}}}{B_{b}}\right)^{2}\right] .
\end{aligned}
$$

Foram assumidos os mesmos valores iniciais da reatividade generalizada do caso da APSD, e a reatividade total foi recalculada. A Tabela 5.11 mostra os valores recalculados e a reatividade do modelo da cinética pontual clássica. A Fig. 5.2. mostra gráficos da $\Sigma \rho_{g e n},-\zeta$ e $\rho_{c p c}$. obtidos por CPSD. Como no caso da APSD, a reatividade da cinética pontual clássica se aparta da reatividade generalizada total a partir de certo nível de subcriticalidade e se aproxima do valor negativo do índice de subcriticalidade. 


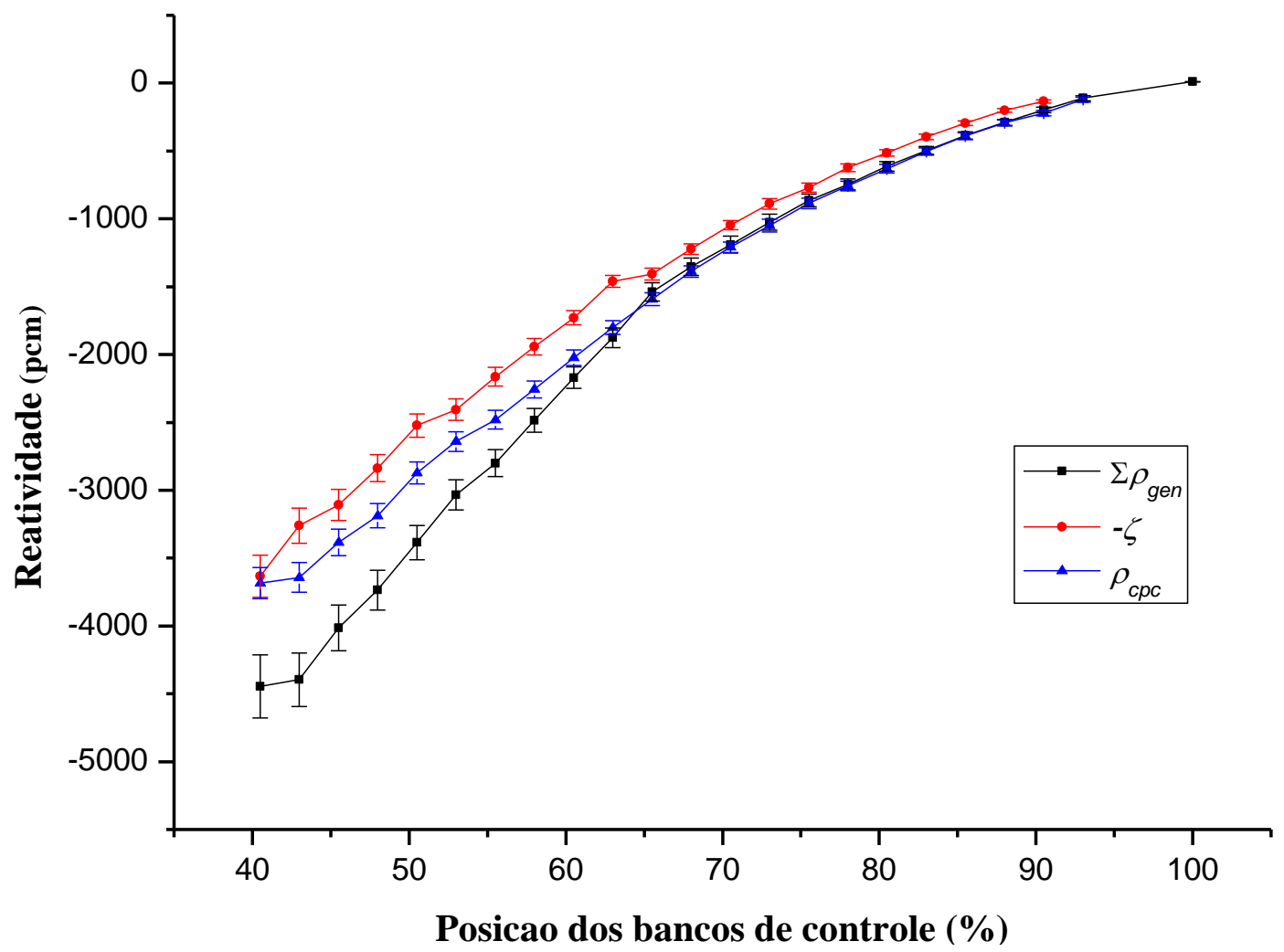

Fig. 5.2. A reatividade generalizada, $\Sigma \rho_{\text {gen }}$, o valor negativo do índice de subcriticalidade, - $\zeta$, e a reatividade da cinética pontual clássica, $\rho_{c p c}$.

A Fig. 5.3. ilustra comparação $\Sigma \rho_{\text {gen }}$ obtidas pela CPSD e por duas APSD correspondentes. As tabelas dos resultados obtidos pelas APSDs encontram-se no Apêndice D. Os gráficos da figura mostram claramente que o método da CPSD é compatível com o da APSD, e que as divergências começam aparecer a partir da posição em que foi inserida a fonte adicional de nêutrons, a saber, da $63.0 \%$.

Quanto às incertezas das reatividades, $\Sigma \rho_{\text {gen }}$, e dos índices de subcriticalidade, $\zeta$, não foram observadas diferenças consideráveis até que os dois detectores de $\mathrm{BF}_{3}$ foram substituídos, i.e., até a posição de $73.0 \%$. Porém, a partir da posição de $70.5 \%$, no sentido da inserção dos BCs, até a posição de 40.5\%, a mais subcrítica, notou-se uma ligeira vantagem da CPSD sobre a APSD2 em relação às incertezas, tanto das $\Sigma \rho_{\text {gen }}$ como dos $\zeta$. Contudo, as incertezas dos $\zeta$, obtidos pela APSD1, na qual o 
detector de ${ }^{3} \mathrm{He}$ de sensibilidade alta foi utilizado, foram levemente menor do que as dos $\zeta$ calculados a partir dos dados da CPSD.

Tabela 5.11. As reatividades generalizadas de CPSD recalculadas, fator de multiplicação efetivo e a reatividade do modelo da cinética pontual clássica.

\begin{tabular}{|c|c|c|c|c|c|c|c|c|}
\hline $\begin{array}{c}\text { Posição } \\
\text { dos BCs }(\%)\end{array}$ & $\begin{array}{c}\boldsymbol{\rho}_{\boldsymbol{g e n}} \\
(\mathrm{pcm})\end{array}$ & $\begin{array}{c}\boldsymbol{\sigma}_{\boldsymbol{\rho g} \boldsymbol{g} \boldsymbol{n}} \\
(\mathrm{pcm})\end{array}$ & $\begin{array}{l}\boldsymbol{\Sigma} \boldsymbol{\rho}_{\boldsymbol{g e n}} \\
(\mathrm{pcm})\end{array}$ & $\begin{array}{c}\boldsymbol{\sigma}_{\boldsymbol{\Sigma} \boldsymbol{\rho}} \\
(\mathrm{pcm})\end{array}$ & $\boldsymbol{k}_{\text {eff }}^{(\mathbf{a})}$ & $\sigma_{k e f f}$ & $\begin{array}{c}\boldsymbol{\rho}_{c p c} \\
(\mathrm{pcm})\end{array}$ & $\begin{array}{c}\boldsymbol{\sigma}_{\boldsymbol{\rho c p c}} \\
(\mathrm{pcm})\end{array}$ \\
\hline 100,0 & 10,00 & 2,97 & 10,00 & 2,97 & 1,00010 & 0,00003 & & \\
\hline 93,0 & $-120,40$ & 18,25 & $-110,40$ & 18,49 & 0,99890 & 0,00018 & $-120,40$ & 18,25 \\
\hline 90,5 & $-86,04$ & 9,05 & $-196,44$ & 20,59 & 0,99804 & 0,00021 & $-219,89$ & 20,39 \\
\hline 88,0 & $-93,23$ & 9,97 & $-289,67$ & 22,87 & 0,99711 & 0,00023 & $-293,33$ & 22,08 \\
\hline 85,5 & $-96,48$ & 11,72 & $-386,15$ & 25,70 & 0,99615 & 0,00026 & $-391,01$ & 24,26 \\
\hline 83,0 & $-110,37$ & 13,93 & $-496,52$ & 29,23 & 0,99506 & 0,00029 & $-505,19$ & 27,20 \\
\hline 80,5 & $-116,67$ & 17,51 & $-613,20$ & 34,08 & 0,99391 & 0,00034 & $-630,37$ & 30,90 \\
\hline 78,0 & $-134,69$ & 21,27 & $-747,89$ & 40,17 & 0,99258 & 0,00040 & $-757,91$ & 34,67 \\
\hline 75,5 & $-116,84$ & 27,21 & $-864,72$ & 48,52 & 0,99143 & 0,00048 & $-886,13$ & 38,96 \\
\hline 73,0 & $-160,94$ & 33,56 & $-1025,67$ & 58,99 & 0,98985 & 0,00058 & $-1050,52$ & 47,87 \\
\hline 70,5 & $-163,88$ & 16,20 & $-1189,55$ & 61,18 & 0,98824 & 0,00060 & $-1209,08$ & 38,24 \\
\hline 68,0 & $-163,79$ & 19,23 & $-1353,34$ & 64,13 & 0,98665 & 0,00062 & $-1386,73$ & 42,12 \\
\hline 65,5 & $-184,31$ & 23,38 & $-1537,65$ & 68,26 & 0,98486 & 0,00066 & $-1591,84$ & 47,00 \\
\hline 63,0 & $-338,95$ & 25,25 & $-1876,60$ & 72,78 & 0,98158 & 0,00070 & $-1800,04$ & 50,96 \\
\hline 60,5 & $-294,27$ & 30,50 & $-2170,88$ & 78,91 & 0,97875 & 0,00076 & $-2023,53$ & 56,60 \\
\hline 58,0 & $-312,99$ & 37,73 & $-2483,87$ & 87,47 & 0,97576 & 0,00083 & $-2255,99$ & 62,20 \\
\hline 55,5 & $-316,05$ & 44,53 & $-2799,92$ & 98,15 & 0,97276 & 0,00093 & $-2480,34$ & 68,12 \\
\hline 53,0 & $-234,77$ & 53,31 & $-3034,69$ & 111,69 & 0,97055 & 0,00105 & $-2640,70$ & 73,28 \\
\hline 50,5 & $-351,08$ & 60,65 & $-3385,77$ & 127,10 & 0,96725 & 0,00119 & $-2873,03$ & 80,04 \\
\hline 48,0 & $-350,07$ & 72,88 & $-3735,84$ & 146,51 & 0,96399 & 0,00136 & $-3187,98$ & 88,78 \\
\hline 45,5 & $-277,87$ & 84,82 & $-4013,71$ & 169,29 & 0,96141 & 0,00156 & $-3385,11$ & 97,97 \\
\hline 43,0 & $-382,04$ & 100,80 & $-4395,76$ & 197,03 & 0,95789 & 0,00181 & $-3643,06$ & 108,90 \\
\hline 40,5 & $-50,20$ & 125,46 & $-4445,95$ & 233,58 & 0,95743 & 0,00214 & $-3683,31$ & 114,12 \\
\hline
\end{tabular}

(a)Calculado pela fórmula: $k_{\text {eff }}=1 /\left(1-\Sigma \rho_{\text {gen }}\right)$. 


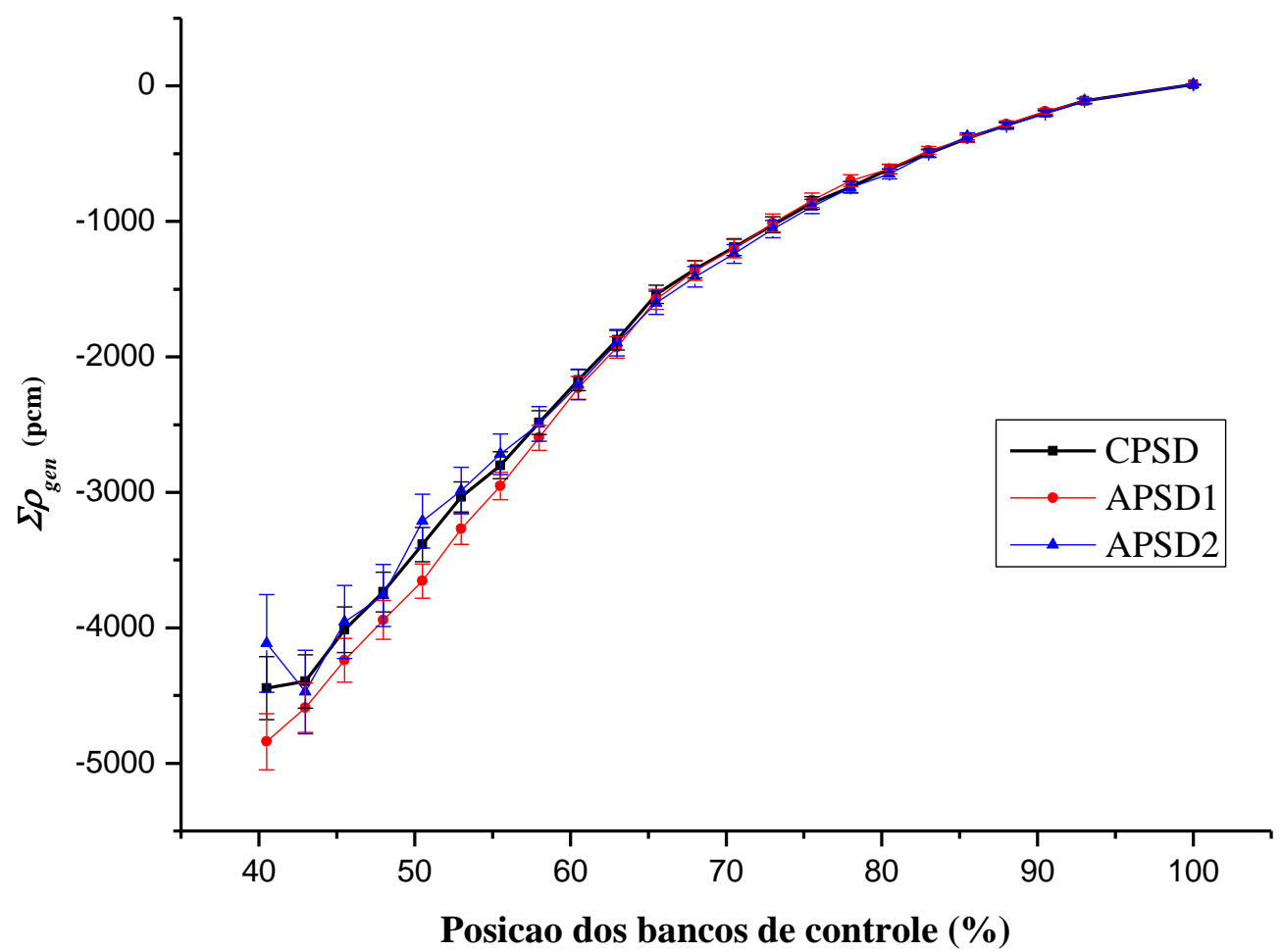

(a)

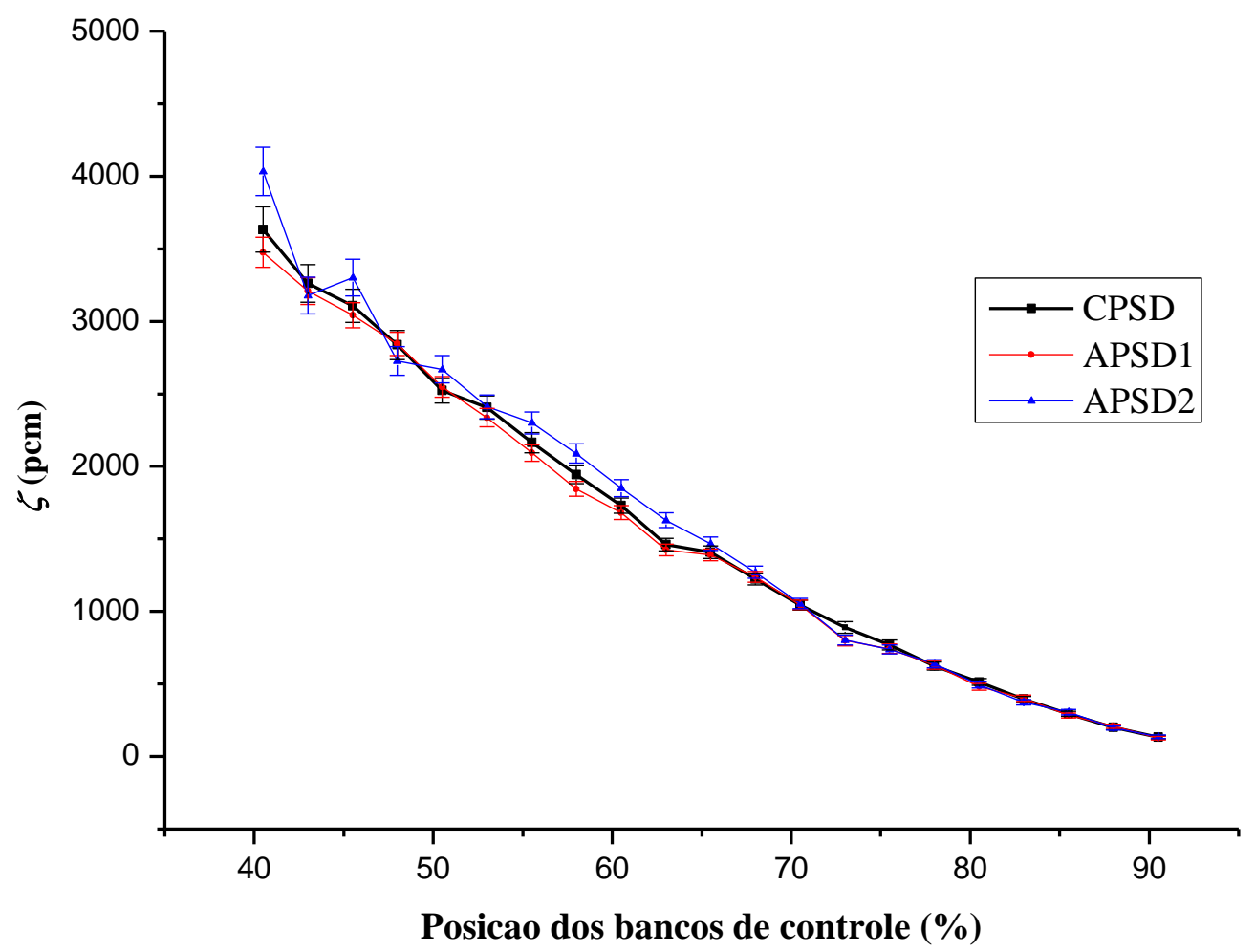

(b)

Fig. 5.3. A $\Sigma \rho_{\text {gen }}$ (a) e o $\zeta$ (b) da CPSD e de duas APSD correspondentes. 
A fim de demonstrar a consistência quando os detectores foram alterados e quando a fonte adicional de Am-Be foi inserida, Tabelas 5.12 e 5.13 mostram os dados experimentais que foram medidos antes e depois de qualquer mudança, seja do detector ou da fonte adicional. A Tabela 5.12 mostra as contagens do detector, $R$, e as constantes de decaimento de nêutrons prontos, $B$; e a Tabela 5.13, as potências relativas, $P_{N}$, as reatividades generalizadas parciais $\rho_{g e n}$ e os índice de subcriticalidade, $\zeta$.

Da Tabela 5.12, fica evidente que, quando os detectores de sensibilidade baixa são substituídos por detectores de sensibilidade superior, as contagens aumentam notavelmente. $R_{1}$ é a contagem no detector na posição 1 , onde o de $\mathrm{BF}_{3}$ foi substituído pelo detector de ${ }^{3} \mathrm{He}$ de sensibilidade alta; ao passo que, $R_{2}$ é da posição 2 , na qual outro de $\mathrm{BF}_{3}$ foi trocado pelo detector de ${ }^{3} \mathrm{He}$ de sensibilidade média. A constante de decaimento de nêutrons prontos $(B)$ mantém consistência entre os dados experimentais e seus valores variam dentro do limite da incerteza experimental 3- $\sigma$. O mesmo acontece com a potência relativa $\left(P_{N}\right)$ na Tabela 5.13. Além disso, nota-se que as incertezas experimentais dessas duas últimas grandezas, $B$ e $P_{N}$, diminuem nas medidas com detectores mais sensíveis.

Entretanto, na Tabela 5.13 observa-se que a inserção da fonte adicional de 1Ci no refletor do sistema causa maiores discrepâncias nos parâmetros como: $P_{N}, \rho_{\text {gen }} \mathrm{e} \zeta$. Comparando os valores destas grandezas nas posições $68,0 \%$ e $65,5 \%$, pode-se concluir que as discrepâncias ficam além do limite da incerteza experimental 3- $\sigma$. Isso pode ser causado pela redistribuição da densidade de potência no sistema, impelida pela presença da fonte adicional de nêutrons. A fonte intrínseca ou espontânea referese à fonte distribuída que ocupa o espaço de todos combustíveis. Neste caso, a densidade de fissão assume o modo fundamental; no entanto, quando a fonte adicional de Am-Be é inserida no refletor, a distribuição do modo fundamental é deformada e a densidade de fissão deslocada na direção do local dessa fonte. Conseqüentemente, o 
fator de multiplicação, que é a função da distribuição da densidade de fissão muda em relação ao caso anterior.

Tabela 5.12: Contagens no Detector na posição 1 e 2, e Constante de Decaimento de Nêutrons Prontos nas posições de sobreposição de medidas.

\begin{tabular}{cccccccc}
\hline Detector & Posição dos BCs & $\mathbf{R}_{\mathbf{1}}{ }^{(\mathbf{c})}$ & $\boldsymbol{\sigma}_{\mathbf{R} \mathbf{1}}$ & $\mathbf{R}_{\mathbf{2}}{ }^{(\mathbf{d})}$ & $\boldsymbol{\sigma}_{\mathbf{R} 2}$ & $\mathbf{B}\left(\mathrm{s}^{-1}\right)$ & $\boldsymbol{\sigma}_{\mathbf{B}}\left(\mathrm{s}^{-1}\right)$ \\
$(\mathbf{F o n t e})$ & $(\%)$ & & & & & & \\
\hline $\mathbf{B F}_{\mathbf{3}}$ & 78,0 & 1899,57 & 2,01 & 2004,68 & 2,11 & $-470,928$ & 6,482 \\
$\left(\mathbf{F F E}^{(\mathbf{a})}\right)$ & 75,5 & 1597,41 & 1,75 & 1694,22 & 1,87 & $-510,971$ & 7,743 \\
& 73,0 & 1363,21 & 1,51 & 1454,34 & 1,64 & $-562,312$ & 10,833 \\
\hline${ }^{3} \mathbf{H e}$ & 78,0 & 14163,86 & 10,56 & 5658,78 & 4,68 & $-465,010$ & 2,713 \\
& 75,5 & 12097,27 & 8,96 & 4847,63 & 4,08 & $-511,644$ & 2,585 \\
$(\mathbf{F F E})$ & 73,0 & 10431,70 & 7,80 & 4205,00 & 3,51 & $-556,072$ & 3,600 \\
\hline & & & & & & & \\
\hline${ }^{3} \mathbf{H e}$ & 70,5 & 9080,15 & 6,50 & 3693,64 & 3,04 & $-611,830$ & 4,169 \\
$(\mathbf{F F E})$ & 68,0 & 8028,29 & 5,90 & 3294,63 & 2,63 & $-667,311$ & 4,947 \\
& 65,5 & 7039,01 & 5,24 & 2924,16 & 2,61 & $-731,369$ & 6,124 \\
\hline${ }^{3} \mathbf{H e}$ & 70,5 & 66654,76 & 13,16 & 31172,59 & 8,79 & $-616,854$ & 4,580 \\
$\left(\mathbf{1 C i}{ }^{(\mathbf{b})}\right)$ & 68,0 & 59498,15 & 12,51 & 27310,66 & 7,83 & $-664,630$ & 4,969 \\
& 65,5 & 52841,05 & 11,82 & 24005,87 & 6,95 & $-723,931$ & 5,649 \\
\hline
\end{tabular}

(a) Fonte de Fissão Espontânea , ${ }^{\text {(b) }}$ Fonte de Am-Be ( 1 Curie), ${ }^{(c)}$ Contagem no detector da posição $1,{ }^{(d)}$ Contagem no detector da posição 2.

Tabela 5.13: $\rho_{\text {gen }}, \Sigma \rho_{\text {gen }}$, e $\zeta$ nas posições sobrepostas.

\begin{tabular}{cccccccc}
\hline $\begin{array}{c}\text { Detector } \\
\text { (Fonte) }\end{array}$ & $\begin{array}{c}\text { Posição dos } \\
\text { BCs }(\%)\end{array}$ & $\boldsymbol{P}_{N}$ & $\boldsymbol{\sigma}_{\boldsymbol{P N}}$ & $\boldsymbol{\rho}_{\boldsymbol{g e n}}(\mathrm{pcm})$ & $\boldsymbol{\sigma}_{\boldsymbol{\rho g e n}}(\mathrm{pcm})$ & $\boldsymbol{\zeta}(\mathrm{pcm})$ & $\boldsymbol{\sigma}_{\boldsymbol{\zeta}}(\mathrm{pcm})$ \\
\hline $\mathbf{B F}_{\mathbf{3}}\left(\mathbf{F F E}^{(\mathbf{a})}\right)$ & 75,5 & 0,868 & 0,036 & $-116,838$ & 27,208 & 769,291 & 33,727 \\
& 73,0 & 0,847 & 0,042 & $-160,941$ & 33,556 & 889,583 & 39,219 \\
\hline${ }^{\mathbf{3}} \mathbf{H e}(\mathbf{F F E})$ & 75,5 & 0,831 & 0,013 & $-150,528$ & 11,313 & 737,756 & 26,516 \\
& 73,0 & 0,854 & 0,014 & $-150,366$ & 12,277 & 880,176 & 29,390 \\
\hline & & & & & & & \\
\hline${ }^{\mathbf{3}} \mathbf{H e}(\mathbf{F F E})$ & 68,0 & 0,882 & 0,018 & $-163,795$ & 19,229 & 1222,936 & 38,566 \\
& 65,5 & 0,884 & 0,020 & $-184,307$ & 23,385 & 1407,535 & 43,699 \\
\hline${ }^{\mathbf{3}} \mathbf{H e}\left(\mathbf{1 C i}{ }^{(\mathbf{b})}\right)$ & 68,0 & 0,805 & 0,017 & $-268,224$ & 19,196 & 1109,920 & 35,643 \\
& 65,5 & 0,812 & 0,018 & $-294,359$ & 21,644 & 1273,669 & 39,682 \\
\hline
\end{tabular}

${ }^{\text {(a) }}$ Fonte de Fissão Espontânea, ${ }^{(b)}$ Fonte de Am-Be ( 1 Curie). 


\section{ANÁLISE TEÓRICA}

As análises teóricas aplicadas às medidas subcríticas foram realizadas, primeiro, numa abordagem estocástica utilizando MCNP5 (MCNP-5 X-5 Monte Carlo Team, 2003), junto com sua biblioteca de dados nucleares ENDF/B-VII.0 (Oblozinsky e Herman, 2006), e, segundo, num método determinístico baseado em sistemas NJOY/AMPX-II/TORT acoplados (Dos Santos, et al., 2000). A metodologia determinística mencionada será referida como GPT-TORT. O modelo geométrico e os dados materiais e geométricos aplicados nesta análise teórica surgiram da LEU.COMP.THERM.082. A única exceção é a posição dos bancos de controle BC1 e BC2, que, na presente análise, são inseridos no núcleo para variar a reatividade do sistema e a presença da fonte adicional de nêutrons de Am-Be.

\subsection{Método Estocástico: MCNP (Monte Carlo N-Particle Transport Code) (MCNP-5 X-5 Monte Carlo Team, 2003)}

O método de Monte Carlo é um método numérico que faz simulação das partículas individuais e, através de contadores (ou "tallies"), registra alguns "aspectos" específicos de seu comportamento médio, sendo estes aspectos previamente requerido pelo usuário. O método trata com problemas de transporte por meio de simulação de histórias individuais das partículas. A partir do comportamento médio das partículas simuladas pode-se inferir o comportamento médio das partículas do sistema físico.

MCNP é um código computacional baseado no método de Monte Carlo que se aplica, principalmente, no transporte de partículas (nêutrons, fótons e elétrons), lidando com energia contínua ou discreta, dependendo do problema; com geometria tridimensional completa; e com dependência do tempo. Uma das vantagens do código é o uso as seções de choque pontuais que constituem uma biblioteca de dados 
acoplados ao programa; que no caso de nêutrons, podem-se citar as bibliotecas de ENDF/B-VII. O código é um recurso muito poderoso para trabalhar com geometria que não pode ser representada por formas regulares como cubos, cilindros e esferas. Uma representação mais detalhada do sistema em todos os aspectos de dados físicos é possível por não lidar com homogeneização no espaço, na energia e no tempo. Os dados nucleares avaliados a partir da biblioteca ENDF é previamente convertido no formato ACE (A Compact ENDF) através do código NJOY (MacFarlane, 1994) para serem acessados pelo MCNP.

O reator IPEN/MB-01 foi modelado com os recursos geométricos do código MCNP5.

\subsubsection{Cálculo da Criticalidade pelo MCNP}

Através de cálculos da criticalidade obtém-se o fator de multiplicação efetivo, $k_{\text {eff }}$, que consiste em número médio de nêutrons de fissão produzido numa geração por nêutron da geração anterior. O cálculo da criticalidade se baseia no método iterativo de potência (Brown, 2005; Nakamura, 1986). Ou seja, uma vez estabelecido o valor inicial para $k_{\text {eff }}$ e a distribuição espacial inicial de nêutrons da fissão, história desses nêutrons são seguidas até que ocorra absorção seguida de fissão, de tal forma que, um novo valor para $k_{\text {eff }}$ é estimado e os pontos nos quais as fissões ocorreram são armazenados para a segunda geração. Essa nova distribuição de nêutrons de fissão é usadoa para seguir histórias da segunda geração, gerando, assim, outra distribuição de nêutrons da fissão e nova estimativa do fator de multiplicação. O mesmo procedimento é repetido até que a distribuição espacial converge.

Para calcular $k_{\text {eff }}$ no código Monte Carlo, precisa-se do cartão KCODE, que contém informações como: o número nominal de histórias de nêutrons da fonte, valor 
inicial de $k_{\text {eff, }}$ o número total de iteração, entre outras. A distribuição espacial inicial de nêutrons da fonte pode ser fornecida por cartão KSRC ou por SDEF. O KSRC fornece um conjunto de posições dos pontos em ( $\mathrm{x}, \mathrm{y}, \mathrm{z})$, ao passo que o SDEF, uma distribuição uniforme de pontos no volume. A distribuição pode ser provida pelo arquivo SRCRT, proveniente de um cálculo prévio.

O esquema de cálculo do MCNP5 segue seu caminho padrão e uma "rodada" de KCODE foi requerida para cada posição dos bancos de controle. A reatividade entre os passos de inserção do banco de controle obteve-se por:

$$
\rho=\frac{\left(k_{i+1}-k_{i}\right)}{\left(k_{i+1} \cdot k_{i}\right)},
$$

onde $\rho$ é a reatividade entre dois estados, $k$ é o fator de multiplicação efetivo, e os subíndices $i$ e $i+1$ indicam os dois estados consecutivos. A reatividade total é a soma das reatividades parciais entre os estados.

Além disso, o índice de subcriticalidade ( $\zeta$ é calculado pela razão de número de nêutrons emitidos por fonte para número de nêutrons emitidos por reação (n, xn) somado por número de nêutrons da fissão, ou seja:

$$
\zeta=\frac{N^{o} \text { de } n \text { da fonte }}{N^{o} \text { de } n \text { de }(n, x n)+N^{o} \text { de } n \text { da fissao }} .
$$

\subsection{Método Determinístico: GPT-TORT (Rhoades, 1991)}

TORT é um código determinístico de transporte de radiação que calcula fluxo de nêutrons e/ou de fótons através de geometrias bi ou tridimensionais devido às 
partículas que incidem nas fronteiras dos sistemas, devido às fontes internas fixas ou geradas internamente por interação com materiais dos sistemas. O problema de criticalidade, ou de autovalor de reator, pode ser solucionado, também.

No código TORT, o transporte é representado pela equação de Boltzmann, porém, a dependência no tempo não é tratada. As variáveis angular e da energia são tratadas pelo método de ordenadas discretas e o formalismo de multigrupo, respectivamente. O espalhamento anisotrópico é tratado usando expansão de Legendre, de ordem arbitrária; e as variáveis espaciais, pelo método de diferenças pesadas, nodal ou das características. Tanto a geometria cilíndrica quanto a cartesiana são suportadas no TORT, além de várias geometrias bidimensionais. Um método de sobreposição de corpo auxilia na especificação de regiões matériais.

\subsubsection{Método de Ordenadas Discretas (Carlson e Bell, 1958),}

O método de ordenadas discretas é o principal método determinístico que consiste numa técnica numérica de solução da equação de transporte linear, que avalia a equação num conjunto específico de ângulos discretos; e a integral na direção é aproximada por uma somatória ponderada dos resultados em todos os ângulos predeterminados. Por exemplo, para uma função qualquer de variável angular, $F(\Omega)$, a integral é aproximada por seguinte fórmula de quadratura:

$$
\int F(\vec{\Omega}) d \vec{\Omega} \cong \sum_{m=1}^{M} W_{m} F\left(\vec{\Omega}_{m}\right),
$$

onde $W_{m}$ são os pesos de quadratura.

Para ilustrar as discretizações das variáveis, considere a equação de transporte, 
em estado estacionário, dada por:

$\vec{\nabla} \cdot \vec{\Omega} \Phi(\vec{r}, E, \vec{\Omega})+\Sigma_{t} \Phi(\vec{r}, E, \vec{\Omega})=Q(\vec{r}, E, \vec{\Omega})$

onde o termo $Q(\vec{r}, E, \vec{\Omega})$ inclui espalhamento, fonte de fissão e fonte externa; e um mesh posicionado em $(i, j, k)$, cujo volume, $\Delta V$, é dado por: $\Delta V=2 a \times 2 b \times 2 c$, onde $a$, $b$ e $c$ são as distâncias entre o centro do mesh e as superfícies perpendiculares ao eixo $x, y$ e $z$, respectivamente.

Agora, integra-se a Eq. (6.3) no espaço da fase, em todo o volume do mesh, $\Delta V$

$$
\iint_{\Delta V \Omega E}\left(\vec{\nabla} \cdot \vec{\Omega} \Phi(\vec{r}, E, \vec{\Omega})+\Sigma_{t} \Phi(\vec{r}, E, \vec{\Omega})\right) d E d \Omega d V=\iiint_{\Delta V \Omega E} Q(\vec{r}, E, \vec{\Omega}) d E d \Omega d V
$$

No formalismo multigrupo, a Eq. (6.4) pode ser escrita por:

$$
\int_{\Delta V} \int_{\Omega} \sum_{g=1}^{G}\left(\vec{\nabla} \cdot \vec{\Omega} \Phi_{g}(\vec{r}, \vec{\Omega})+\Sigma_{g}^{t} \Phi_{g}(\vec{r}, \vec{\Omega})\right) d \Omega d V=\int_{\Delta V} \int_{\Omega} \sum_{g=1}^{G} Q_{g}(\vec{r}, \vec{\Omega}) d \Omega d V
$$

Aplicando a discretização das direções: $\vec{\Omega}=\left\{\vec{\Omega}_{m}\right\}$, a Eq. (6.5) é reescrita da seguinte forma:

$$
\int_{\Delta V} \sum_{m=1}^{M} \sum_{g=1}^{G} W_{m}\left(\vec{\nabla} \cdot \vec{\Omega}_{m} \Phi_{g, m}(\vec{r})+\Sigma_{g, m}^{t} \Phi_{g, m}(\vec{r})\right) d V=\int_{\Delta V} \sum_{m=1}^{M} \sum_{g=1}^{G} W_{m} Q_{g, m}(\vec{r}) d V
$$

Considere o primeiro termo do lado esquerdo da Eq. (6.6);

$$
\int_{\Delta V} \vec{\nabla} \cdot \vec{\Omega}_{m} \Phi_{g, m}(\vec{r}) d V=\oint \vec{\Omega}_{m} \Phi_{g, m}(\vec{r}) \cdot \hat{n} d A=\int_{i-a, j, k}^{i+a, j, k} \mu_{m} \Phi_{g, m}(\vec{r}) d A_{i}+\int_{i, j-b, k}^{i, j+b, k} \eta_{m} \Phi_{g, m}(\vec{r}) d A_{j}+\int_{i, j, k-c}^{i, j, k+c} \xi_{m} \Phi_{g, m}(\vec{r}) d A_{k}
$$


onde $\mu_{m}, \eta_{m}$ e $\xi_{m}$ são os cossenos dos ângulos correspondentes.

Desta maneira, obtém a expressão final, dada por:

$$
\begin{aligned}
\mu_{m} & \left(\Phi_{i+a, j, k . g, m} \cdot A_{i+a, j, k . g, m}-\Phi_{i-a, j, k . g, m} \cdot A_{i-a, j, k . g, m}\right) \\
& +\eta_{m}\left(\Phi_{i, j+b, k . g, m} \cdot A_{i, j+b, k . g, m}-\Phi_{i, j-b, k . g, m} \cdot A_{i, j-b, k . g, m}\right) \\
& +\xi_{m}\left(\Phi_{i, j, k+c . g, m} \cdot A_{i, j, k+c . g, m}-\Phi_{i, j, k-c . g, m} \cdot A_{i, j, k-c . g, m}\right) \\
& +\sum_{i, j, k, g, m}^{t} \Phi_{i, j, k, g, m} \Delta V_{i, j, k}=Q_{i, j, k, g, m} \Delta V_{i, j, k} .
\end{aligned}
$$

Pode-se notar que no problema representado pela Eq. (6.7) tem sete incógnitas, uma equação e três condições de contorno. As três relações necessárias para resolver o problema são obtidas pelo método de diferenças pesadas, a saber:

$$
\Phi_{i, j, k, g, m}=\gamma^{m} \Phi_{i+a, j, k, g, m}+\left(1-\gamma^{m}\right) \Phi_{i-a, j, k, g, m},
$$

onde $\gamma^{m}$ é fator peso e $(1 / 2) \leq \gamma^{m} \leq 1$.

Dos métodos mais simples pode-se citar o modelo de diferenças lineares ou de "diamond", no qual o fluxo varia linearmente dentro do volume $\Delta V$. Por exemplo, o componente do fluxo em $(i, j, k)$ na direção do eixo $x$ é dado por:

$$
\Phi_{i, j, k, g, m}=\frac{1}{2}\left(\Phi_{i+a, j, k, g, m}+\Phi_{i-a, j, k, g, m}\right) .
$$

Como já visto, os ângulos discretos, $\vec{\Omega}_{m}$, são representados pelos cossenos $\mu_{m}$, $\eta_{m}$ e $\xi_{m}$. Portanto, fica evidente que: $\mu_{m}^{2}+\eta_{m}^{2}+\xi_{m}^{2}=1$. Além deste vínculo, para um conjunto de quadratura direcional, são, geralmente, impostas algumas condições de simetria, como por exemplo: 


$$
\begin{aligned}
\sum_{m=1}^{M} W_{m} & =1, & \sum_{m=1}^{M} W_{m} \eta_{m} & =0, \\
\sum_{m=1}^{M} W_{m} \mu_{m} & =0, & \sum_{m=1}^{M} W_{m} \xi_{m} & =0 .
\end{aligned}
$$

Ou seja, para cada par de $\left(W_{m}, \mu_{m}\right),\left(W_{m}, \eta_{m}\right)$ e $\left(W_{m}, \xi_{m}\right)$ sempre existe um $\left(W_{m},-\mu_{m}\right)$, $\left(W_{m},-\eta_{m}\right)$ e $\left(W_{m},-\xi_{m}\right)$.

Enquanto o método Monte Carlo simula partículas individuais, seguindo suas histórias, e fornece informações sobre os comportamentos médios dessas partículas individuais em um determinado volume, os métodos determinísticos resolvem a equação de transporte para tal fim. Como já mencionado anteriormente, o Monte Carlo dá informações específicas requisitadas pelo usuário. Porém, os métodos determinísticos fornecem normalmente informações mais completas, como por exemplo, o fluxo no ponto. O que poderia chamar-se da "solução" da equação de transporte pelo Monte Carlo, é concernente à equação da densidade de probabilidade das partículas no espaço da fase.

Uma das vantagens de usar TORT sobre o uso de MCNP é o fato de lidar com funções adjuntas, ou seja, obtêm-se através do TORT, além de fluxos "diretos", o fluxos adjuntos, para todo espaço da fase discretizado. Desta maneira, pode-se calcular os parâmetros cinéticos como $l_{e f f}, \alpha_{p d}, \zeta, \rho_{\text {gen }}$ e $\rho_{\text {source }}$ de maneira mais direta pelas respectivas definições.

\subsubsection{Geração de Seções de Choque.}

A metodologia aplicada no cálculo determinístico é esquematizada na Fig. 6.1. De uma forma resumida, o programa NJOY (MacFarlane, 1994) acessa a biblioteca de dados nucleares, ENDF/B-VII.0, e processa os arquivos dos dados nucleares numa 
estrutura de grupo fino de energia de nêutrons. O NJOY acessa as bibliotecas básicas, efetua interpolações, alargamento das ressonâncias e a ponderação pelo espectro típico do problema. O módulo LEAPR do sistema NJOY fornece arquivos de espalhamento de nêutrons térmicos $S(\alpha \cdot \beta)$, necessários para ligação de hidrogênio do moderador (água). O módulo RECONR constrói seções de choque pontuais a partir dos parâmetros de ressonância e das leis de interpolação da ENDF. O módulo BROADR aplica o alargamento Doppler nas seções de choque. O UNRESR calcula os efeitos de autoblindagem na região de ressonâncias não resolvidas. O THERMR constrói a matriz de espalhamento na região térmica. E o GROUPR transforma essas seções de choque pontuais em parâmetros de multigrupo. Os módulos RECONR e BROADR de NJOY foram executados com tolerância de interpolação de $0,5 \%$ e $0,2 \%$, respectivamente, para todos os nuclídeos.

O próximo passo foi a produção do conjunto da biblioteca de grupo amplo de energia usando o pacote AMPX-II (Greene, 1976). As seções de choque pontuais e de grupo fino que foram produzidos nas etapas anteriores são transferidos ao AMPX-II por dois módulos de interface AMPXR e BRDROL. O tratamento de autoblindagem da ressonância resolvida na região de energia de nêutrons de $0.625 \mathrm{eV}$ a $5.53 \mathrm{keV}$ é executada pelo módulo ROLAIDS, e do espectro de nêutrons em várias regiões do reator IPEN/MB-01, pelo módulo XSDRNPM.

Em primeiro lugar, considere um arranjo infinito de células de pino de combustível. O cálculo espectral de $K_{\text {inf }}$ é executado pelo XSDRNPM na estrutura de grupos finos, levando em consideração uma condição de contorno de albedo na fronteira externa da célula unitária cilíndrica. As seções de choque são homogeneizadas em nível de grupo fino. Em seguida, estes dados foram fundidos com os de outras regiões tais como refletores radial, superior e inferior e assim por diante. Finalmente, o módulo XSDRNPM considera "fatias" radiais e axiais do reator IPEN/MB-01 para obter o espectro final para o grupo amplo, formatando para todas as regiões, as quais são consideradas na configuração geométrica tridimensional tratada 
pelo código TORT (Rhoades, 1991).

As seções de choque de grupo amplo das barras de controles, dos tubos guia e dos tampões inferiores das barras de controle foram obtidas pelo modelo de supercélula. A esta altura, a biblioteca de seções de choque passa a depender de problema. Uma estrutura de grupo fino com 620 grupos é considerada para gerar a biblioteca de grupo amplo, que por sua vez, é colapsada para 16 grupos amplos com 5 upscattering (Dos Santos, 2005).

A ordem de espalhamento, i.e., a de expansão de Legendre, é $P_{3}$ ao longo de todas as análises. Finalmente, a biblioteca de grupo amplo é convenientemente convertida no formato do código TORT pelo programa GIP (Rhoades, 1975). O TORT executa cálculos de modo de fonte fixa para obter fluxos diretos e fluxos adjuntos levando em consideração uma modelagem geométrica totalmente tridimensional do núcleo do reator IPEN/MB-01.

O programa GIP gera bibliotecas de seções de choque macroscópicas descritas acima para os seguintes para vários materiais constituintes do reator IPEN/MB-01, a saber:

- Núcleo ativo do reator;

- Refletor radial (água);

- Refletor axial inferior (água);

- Alumina inferior;

- Alumina superior;

- Placa Matriz;

- Barra de controle;

- Tampão da barra de controle;

- Tubo guia; e

- Tubo espaçador. 
O cálculo de fluxos diretos considera a fonte intrínseca de $93.41 \%$ a $75.09 \%$; e a fonte pontual de Am-Be, como mostrada na Fig. 3.5 e Fig. 3.7, na face leste do núcleo do reator, de $71.43 \%$ até $1.83 \%$. Em todos os cálculos a mesma fonte de AmBe está presente na posição da fonte de partida do reator. Por outro lado, no cálculo de fluxos adjuntos, assume-se que a fonte é dada por $\gamma \Sigma_{f} / W_{0}$ (Gandini e Salvatores, 2002); onde $\gamma$ é a energia liberada por fissão, $\Sigma_{f}$ é a seção de choque macroscópica de fissão e, $W_{0}$, a potência do reator no estado em consideração.

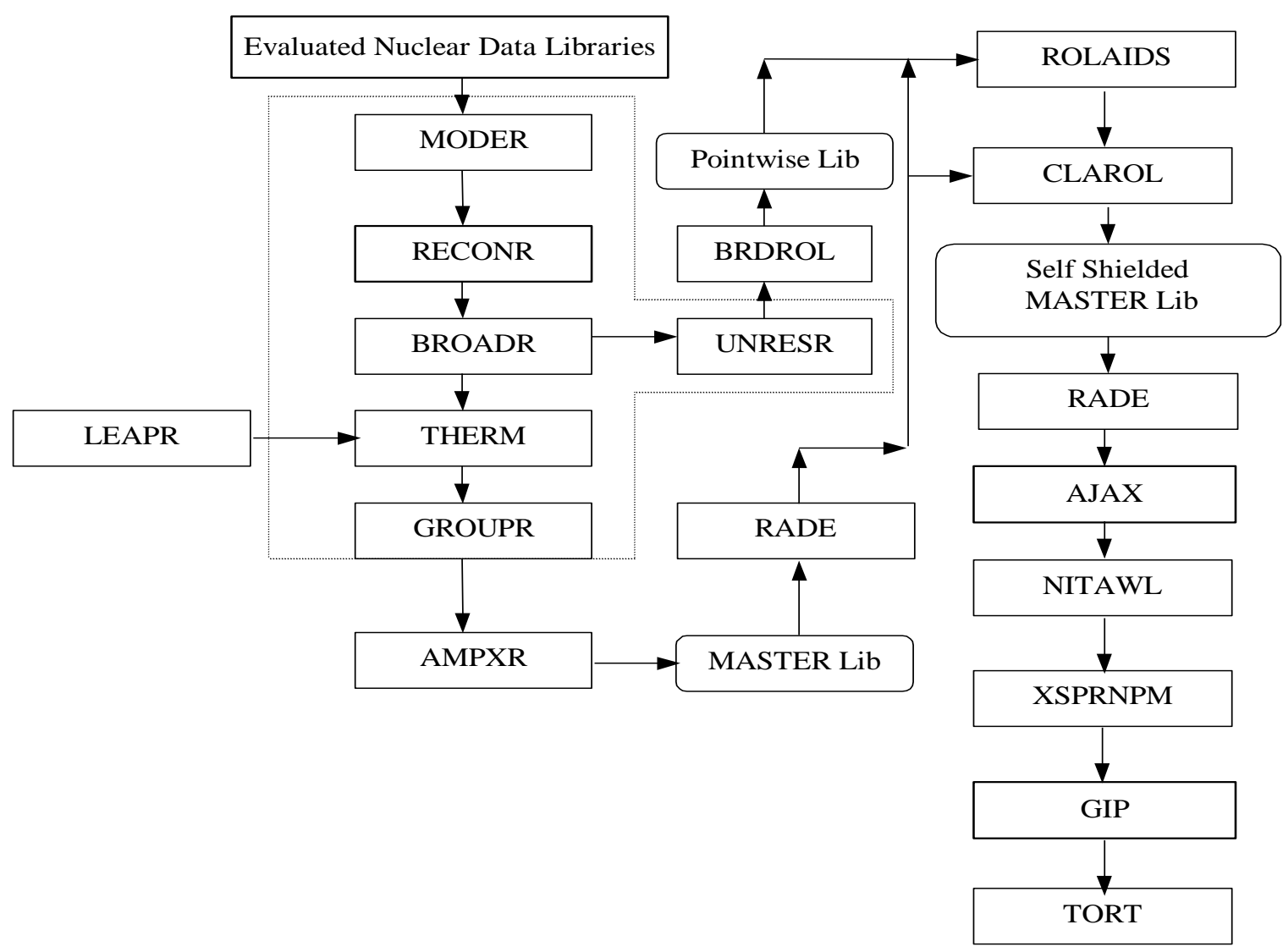

Fig. 6.1. Diagrama da metodologia de cálculo determinístico.

Foram realizados os cálculos do TORT em passos de $2 \mathrm{~cm}$ a partir da posição de $93.41 \%$ retirada. Para cada posição do banco de controle, calcularam-se os fluxos angulares diretos e adjuntos de nêutrons. Em suma, foi considerada a configuração 
geométrica totalmente tri-dimensional em X-Y-Z, a aproximação $P_{3}$, a quadratura angular $S_{16}$, e 16 grupos com 5 upscatterings térmicos (Dos Santos, 2005). A distribuição de mesh consite em: 52 intervalos na direção X, 50 na direção Y, e 81 na direção Z; um total de 210600 intervalos. Esses intervalos foram representados por 10 números de regiões de material. As condições de contorno do problema foram: o vácuo, na parte superior e inferior, e, também, nas bordas laterais do problema. $\mathrm{O}$ critério de convergência para os cálculos direcionados por fonte foi configurado para o limite da $5 \cdot 10^{-4}$.

As grandezas calculadas utilizando os fluxos diretos e adjuntos gerados pelo TORT são dadas nas Eqs. (6.8) a (6.12). Um programa foi elaborado na linguagem Fortran para executar as integrais nessas expressões de maneira numérica. Em particular, a integral no domínio de energia é transformada numa somatória ao longo de todos os grupos de nêutrons. $\Delta \Sigma_{t}(r, E)$ e $\Delta \Sigma_{s} f\left(r, E^{\prime}, \Omega^{\prime} \rightarrow E, \Omega\right)$ são as variações de seções de choque entre as estados.

$$
\begin{aligned}
& \rho_{g e n}=\frac{1}{F}\left\{-\iiint \Delta \Sigma_{t}(r, E) \psi(r, \Omega, E) \psi^{*}(r, \Omega, E) d r d \Omega d E\right. \\
& \left.+\int \ldots \int \Delta \Sigma_{s} f\left(r, E^{\prime}, \Omega^{\prime} \rightarrow E, \Omega\right) \Psi\left(r, \Omega^{\prime}, E^{\prime}\right) \Psi^{*}(r, \Omega, E) d r d \Omega^{\prime} d E^{\prime} d \Omega d E\right\} \\
& \zeta=\frac{\int \ldots \int S(r, \Omega, E) \Psi^{*}(r, \Omega, E) d r d \Omega d E}{F} ; \\
& l_{e f f}=\frac{1}{F} \iiint_{\frac{1}{v(E)}} \Psi(r, \Omega, E) \Psi^{*}(r, \Omega, E) d r d \Omega d E ; \\
& \beta_{e f f_{j}}=\frac{1}{F} \int \ldots \int \chi_{d_{j}}(E) \beta_{j} v \Sigma_{f}\left(r, E^{\prime}\right) \Psi\left(r, \Omega^{\prime}, E^{\prime}\right) \Psi^{*}(r, \Omega, E) d r d \Omega^{\prime} d E^{\prime} d \Omega d E ; \\
& \mathrm{e} \\
& \beta_{e f f}=\sum_{j} \beta_{e f f_{j}},
\end{aligned}
$$


onde:

$F$ : é igual a $\int \cdots \int \chi(E) v \Sigma_{f}\left(r, E^{\prime}\right) \Psi\left(r, \Omega^{\prime}, E^{\prime}\right) \Psi^{*}(r, \Omega, E) d r d \Omega^{\prime} d E^{\prime} d \Omega d E$

$r:$ a coordenada especial;

$\Omega$ : a coordenada angular;

$E$ : a energia de nêutron;

$1 / v$ : o inverso da velocidade de nêutrons;

$\Psi(r, \Omega, E)$ : fluxo angular direto de nêutrons na posição $r$, na direção $\Omega$, e na energia $E$;

$\Psi^{*}(r, \Omega, E)$ : fluxo angular adjunto na posição $r$, na direção $\Omega$, e na energia $E$;

$\chi$ : o espectro de fissão igual a: $(1-\beta) \chi_{p}+\beta \chi_{d}$;

$\beta$ : a fração efetiva de nêutrons atrasados, igual a $\Sigma \beta_{j}$;

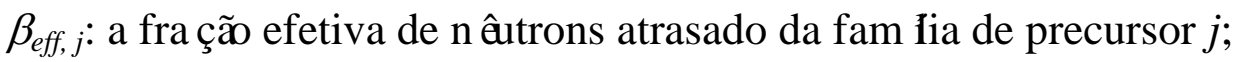

$\chi_{p}:$ o espectro de físsão pronto;

$\chi_{d}$ : o espectro de fissão atrasado;

$v$. número médio de nêutrons produzido por fissão;

$\Sigma f:$ a seção de choque macroscópica de fissão; e

$S(r, \Omega, E)$ : a fonte intrínseca + a fonte de partida ou a fonte na lateral + a fonte de partida.

As variações de seção de choque são ilustrados na Fig. 6.2. Existem dois tipos de tais variações. Em primeiro lugar, quando os bancos de controle se deslocam para iniciar um novo passo, os espaços anteriormente ocupados pelos tampões inferiores de barras de controle passam a ser preenchidos por materiais absorvedores de $\mathrm{Ag}$-In-Cd das barras. Esta variação é representada por $\Delta \Sigma_{X}$ na Fig. 6.2. Em segundo lugar, a água no interior do tubo guia é agora substituída pelo material do tampão inferior. Esta é representada por $\Delta \Sigma_{Y}$. Portanto, as variações de seção de choque são a diferença entre as seção de choque da célula contendo a barra de controle e a da célula do tampão inferior, e a mesma entre esta última e a da célula do tubo guia. Estas são as únicas variações de seção de choque nos cálculos do GPTTORT. 


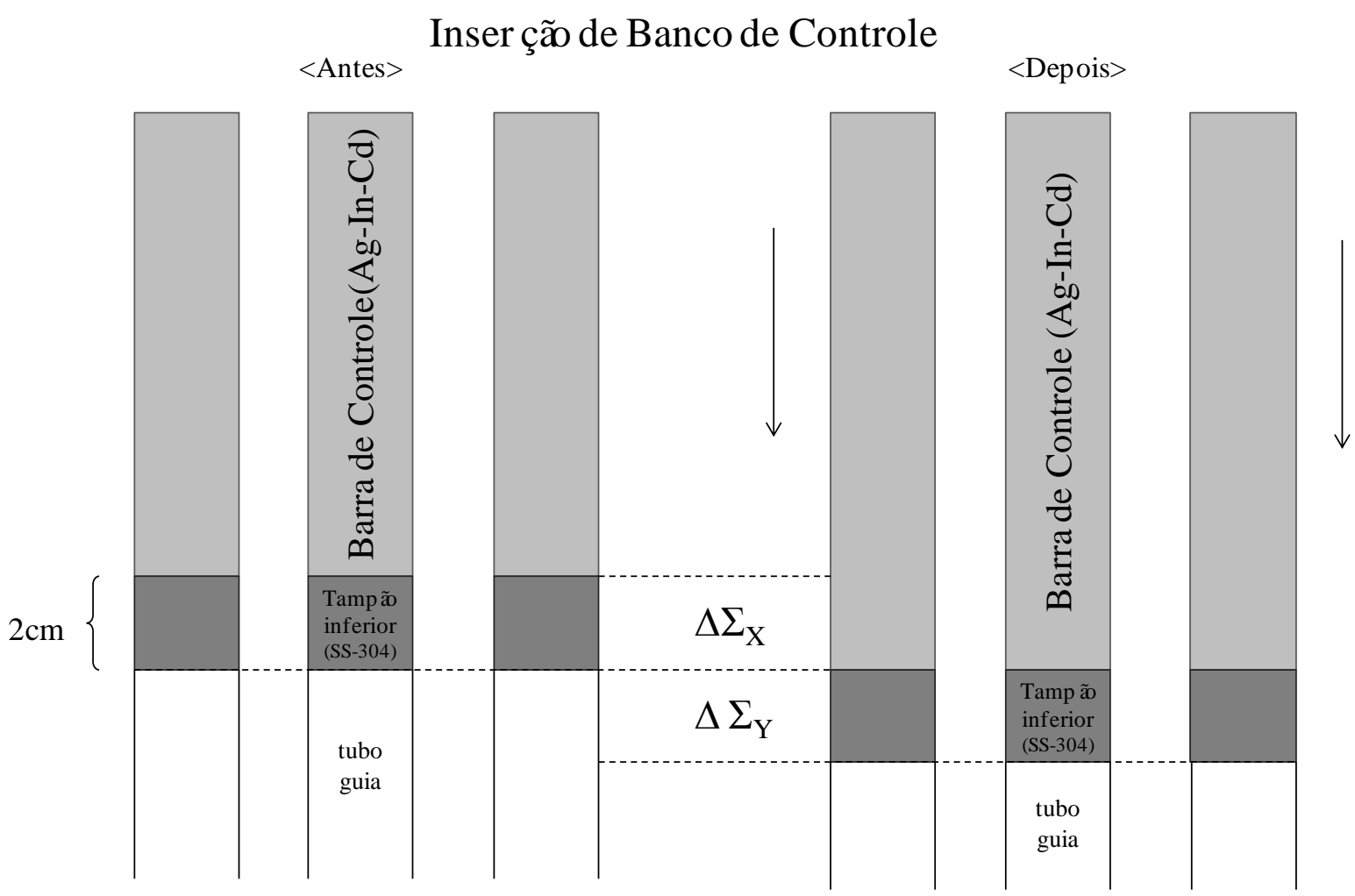

Fig. 6.2. Diagrama da variações de seção de choque. 


\section{RESULTADO DA ANÁLISE TEÓRICA}

Os resultados da abordagem teórica baseada nos sistemas acoplados NJOY/AMPX-II/TORT são mostrados na Tabela 7.1, onde a reatividade da perturbação generalizada $\left(\rho_{\text {gen }}\right)$, o índice de subcriticalidade $(\zeta)$, o tempo de geração de nêutrons prontos $\left(l_{\text {eff }}\right)$ e a fração efetiva de nêutrons atrasados $\left(\beta_{\text {eff }}\right)$ são dados em função da posição dos bancos de controle em unidades de $\%$ retirada. O $\Sigma \rho_{\text {gen }}$, na terceira coluna da tabela, é a soma de todos os $\rho_{\text {gen }}$ parciais, até a posição do banco de controle em consideração, menos a reatividade inserida pelos tampões inferiores das vareta de controle nos estados anteriores. A razão desta subtração é o fato de que a reatividade inserida pelos tampões não é cumulativa.

Conforme mostrado na Tabela 7.1, $\beta_{\text {eff }}$ é praticamente constante e $l_{\text {eff }}$ apresenta alguma variação em função da posição do banco de controle. Não há fundamento experimental para questionar se esse comportamento é plausível ou não. O comportamento do $\beta_{\text {eff }}$ sustenta a hipótese de que essa variável é constante na abordagem experimental.

Os cálculos pelo código TORT foram realizados nas posições diferentes das experimentais, uma vez que a inserção das barras de controle pode ter o passo mínimo equivalente ao comprimento do mesh na direção do eixo do $Z$, paralela às barras; que é igual a $1 \mathrm{~cm}$. Neste trabalho, adotou-se os passos de $2 \mathrm{~cm}$, que é equivalente a $3,66 \%$, aproximadamente, do comprimento total da região ativa do núcleo no eixo $Z$. Entretanto, com o MCNP5 não houve essa espécie de restrição e, portanto, os cálculos foram feitos nas mesmas posições do experimento, conforme mostrado na Tabela 7.2. 
Tabela 7.1. Reatividades e parâmetros efetivos dos nêutrons atrasados a partir da abordagem GPT-TORT

\begin{tabular}{ccccccc}
\hline $\begin{array}{c}\text { Posição } \\
\text { dos }\end{array}$ & $\boldsymbol{\rho}_{\text {gen }}(\mathrm{pcm})$ & $\boldsymbol{\Sigma}_{\boldsymbol{g e n}}(\mathrm{pcm})$ & $\boldsymbol{\zeta}(\mathrm{pcm})$ & $\boldsymbol{l}_{\text {eff }}(\mu \mathrm{seg})$. & $\boldsymbol{\beta}_{\text {eff }}(\mathrm{pcm})$ & $\boldsymbol{k}_{\text {eff }}$ \\
BCs $(\%)$ & & & & & & \\
\hline 100,00 & & & & & & 0,99964 \\
93,41 & $-136,68^{(1)}$ & $-136,68$ & 16,78 & 30,58 & 757,37 & 0,99828 \\
89,74 & $-59,12$ & $-195,81$ & 85,42 & 30,64 & 757,48 & 0,99709 \\
86,08 & $-109,31$ & $-305,12$ & 176,57 & 30,74 & 757,58 & 0,99560 \\
82,42 & $-140,69$ & $-445,81$ & 289,56 & 30,88 & 757,71 & 0,99377 \\
78,75 & $-172,87$ & $-618,68$ & 424,38 & 31,04 & 757,82 & 0,99159 \\
75,09 & $-206,31$ & $-824,99$ & 584,74 & 31,23 & 757,97 & 0,98912 \\
71,43 & $-239,77$ & $-1064,76$ & 770,03 & 31,45 & 758,12 & 0,98626 \\
67,77 & $-274,85$ & $-1339,61$ & 980,39 & 31,71 & 758,25 & 0,98311 \\
64,10 & $-312,09$ & $-1651,70$ & 1220,30 & 31,99 & 758,39 & 0,97956 \\
60,44 & $-346,22$ & $-1997,92$ & 1490,46 & 32,31 & 758,49 & 0,97572 \\
56,70 & $-345,85$ & $-2343,76$ & 1793,93 & 32,67 & 758,57 & 0,97147 \\
53,11 & $-535,29$ & $-2879,05$ & 2118,43 & 33,06 & 758,59 & 0,96707 \\
49,45 & $-427,16$ & $-3306,21$ & 2473,41 & 33,48 & 758,57 & 0,96230 \\
45,79 & $-504,79$ & $-3811,00$ & 2868,30 & 33,94 & 758,50 & 0,95731 \\
42,12 & $-529,03$ & $-4340,03$ & 3300,13 & 34,41 & 758,38 & 0,95213 \\
38,46 & $-540,94$ & $-4880,97$ & 3757,25 & 34,90 & 758,24 & 0,94694 \\
34,80 & $-538,08$ & $-5419,05$ & 4236,92 & 35,39 & 758,10 & 0,94178 \\
31,14 & $-520,70$ & $-5939,75$ & 4722,14 & 35,86 & 757,96 & 0,93687 \\
27,47 & $-489,75$ & $-6429,50$ & 5195,83 & 36,30 & 757,84 & 0,93232 \\
23,81 & $-447,29$ & $-6876,79$ & 5641,03 & 36,70 & 757,77 & 0,92829 \\
20,15 & $-396,31$ & $-7273,09$ & 6041,56 & 37,04 & 757,72 & 0,92489 \\
16,48 & $-340,15$ & $-7613,24$ & 6397,24 & 37,33 & 757,71 & 0,92220 \\
12,82 & $-281,88$ & $-7895,12$ & 6681,88 & 37,54 & 757,73 & 0,92016 \\
9,16 & $-224,41$ & $-8119,52$ & 6902,08 & 37,68 & 757,80 & 0,91872 \\
5,49 & $-170,80$ & $-8290,33$ & 7065,76 & 37,77 & 757,89 & 0,91772 \\
1,83 & $-126,88$ & $-8417,21$ & 7174,40 & 37,81 & 758,00 & 0,91715 \\
\hline Valor & & & & &
\end{tabular}

(1) Valor calculado a partir dos $k_{\text {eff. }}$.

A Tabela 7.2 mostra a as reatividades e os índices de subcriticalidade obtidos pelo MCNP5. Neste caso, a reatividade foi obtida com base nos cálculos de $k_{\text {eff }}$ pela Eq. (6.1), e o índice de subcriticaliade, pela Eq. (6.2).

Além disso, obtiveram-se, também, o tempo de geração de nêutrons prontos $\left(l_{e f f},\right)$ e a fração efetiva de nêutrons atrasados $\left(\beta_{\text {eff }}\right)$ utilizando uma versão mais nova do MCNP, a saber, MCNP6, por métodos discutidos em (Kiedrowski, et al., 2009; 
Carluccio, 2011). Os resultados são mostrados na Tabela. 7.3.

Tabela 7.2. Fator de multiplicação efetivo, $k_{\text {eff, }}$ as reatividades e o índice de subcriticalidade, $\zeta$, calculados pelo MCNP5

\begin{tabular}{|c|c|c|c|c|c|c|c|}
\hline $\begin{array}{c}\text { Posição dos } \\
\text { BCs }(\%)\end{array}$ & $\boldsymbol{k}_{\text {eff }}$ & $\sigma_{k e f f}$ & $\boldsymbol{\rho}(\mathrm{pcm})$ & $\boldsymbol{\sigma}_{\rho}(\mathrm{pcm})$ & $\boldsymbol{\Sigma} \boldsymbol{\rho}(\mathrm{pcm})$ & $\boldsymbol{\sigma}_{\Sigma \boldsymbol{\rho}}(\mathrm{pcm})$ & $\boldsymbol{\zeta}(\mathrm{pcm})$ \\
\hline 100,0 & 1,00055 & 0,00003 & & & & & \\
\hline 93,0 & 0,99916 & 0,00003 & $-139,04$ & 4,24 & $-139,04$ & 4,24 & 463,14 \\
\hline 90,5 & 0,99836 & 0,00003 & $-80,20$ & 4,25 & $-219,24$ & 6,01 & 503,73 \\
\hline 88,0 & 0,99750 & 0,00003 & $-86,36$ & 4,26 & $-305,60$ & 6,02 & 552,43 \\
\hline 85,5 & 0,99654 & 0,00003 & $-96,57$ & 4,27 & $-402,17$ & 6,03 & 620,42 \\
\hline 83,0 & 0,99543 & 0,00003 & $-111,90$ & 4,28 & $-514,07$ & 6,04 & 678,61 \\
\hline 80,5 & 0,99407 & 0,00003 & $-137,44$ & 4,29 & $-651,51$ & 6,06 & 750,23 \\
\hline 78,0 & 0,99261 & 0,00003 & $-147,96$ & 4,30 & $-799,47$ & 6,07 & 838,32 \\
\hline 75,5 & 0,99107 & 0,00003 & $-156,54$ & 4,31 & $-956,02$ & 6,09 & 928,25 \\
\hline 73,0 & 0,98936 & 0,00003 & $-174,40$ & 4,33 & $-1130,41$ & 6,11 & 1020,58 \\
\hline 70,5 & 0,98754 & 0,00003 & $-186,28$ & 4,34 & $-1316,69$ & 6,13 & 1141,40 \\
\hline 68,0 & 0,98556 & 0,00003 & $-203,44$ & 4,36 & $-1520,13$ & 6,15 & 1262,15 \\
\hline 65,5 & 0,98344 & 0,00003 & $-218,73$ & 4,38 & $-1738,85$ & 6,18 & 1396,97 \\
\hline 63,0 & 0,98111 & 0,00003 & $-241,49$ & 4,40 & $-1980,34$ & 6,20 & 1533,71 \\
\hline 60,5 & 0,97876 & 0,00003 & $-244,72$ & 4,42 & $-2225,06$ & 6,23 & 1690,73 \\
\hline 58,0 & 0,97619 & 0,00003 & $-268,98$ & 4,44 & $-2494,04$ & 6,26 & 1857,61 \\
\hline 55,5 & 0,97355 & 0,00003 & $-277,79$ & 4,46 & $-2771,83$ & 6,30 & 2032,25 \\
\hline 53,0 & 0,97083 & 0,00002 & $-287,78$ & 3,81 & $-3059,62$ & 5,87 & 2216,44 \\
\hline 50,5 & 0,96793 & 0,00003 & $-308,61$ & 3,84 & $-3368,23$ & 5,41 & 2410,63 \\
\hline 48,0 & 0,96497 & 0,00003 & $-316,91$ & 4,54 & $-3685,13$ & 5,95 & 2618,74 \\
\hline 45,5 & 0,96199 & 0,00003 & $-321,02$ & 4,57 & $-4006,15$ & 6,44 & 2826,13 \\
\hline 43,0 & 0,95888 & 0,00003 & $-337,15$ & 4,60 & $-4343,31$ & 6,48 & 3036,78 \\
\hline 40,5 & 0,95572 & 0,00003 & $-344,82$ & 4,63 & $-4688,13$ & 6,53 & 3243,95 \\
\hline 38,0 & 0,95271 & 0,00003 & $-330,58$ & 4,66 & $-5018,70$ & 6,57 & 3448,09 \\
\hline 35,5 & 0,94968 & 0,00003 & $-334,89$ & 4,69 & $-5353,60$ & 6,61 & 3642,93 \\
\hline 33,0 & 0,94678 & 0,00003 & $-322,53$ & 4,72 & $-5676,13$ & 6,65 & 3832,07 \\
\hline 30,5 & 0,94396 & 0,00003 & $-315,53$ & 4,75 & $-5991,66$ & 6,69 & 4010,97 \\
\hline 28,0 & 0,94128 & 0,00002 & $-301,62$ & 4,05 & $-6293,28$ & 6,24 & 4174,86 \\
\hline 25,5 & 0,93885 & 0,00003 & $-274,97$ & 4,08 & $-6568,26$ & 5,75 & 4323,79 \\
\hline 23,0 & 0,93661 & 0,00003 & $-254,74$ & 4,82 & $-6822,99$ & 6,32 & 4454,59 \\
\hline 20,5 & 0,93474 & 0,00003 & $-213,60$ & 4,85 & $-7036,59$ & 6,84 & 4568,95 \\
\hline 18,0 & 0,93303 & 0,00003 & $-196,07$ & 4,86 & $-7232,66$ & 6,87 & 4673,96 \\
\hline 15,5 & 0,93159 & 0,00003 & $-165,67$ & 4,88 & $-7398,33$ & 6,89 & 4756,36 \\
\hline 13,0 & 0,93042 & 0,00002 & $-134,98$ & 4,16 & $-7533,31$ & 6,41 & 4830,69 \\
\hline 10,5 & 0,92949 & 0,00003 & $-107,54$ & 4,17 & $-7640,85$ & 5,89 & 4890,43 \\
\hline 8,0 & 0,92872 & 0,00003 & $-89,20$ & 4,91 & $-7730,05$ & 6,45 & 4936,12 \\
\hline 5,5 & 0,92816 & 0,00003 & $-64,97$ & 4,92 & $-7795,01$ & 6,96 & 4971,12 \\
\hline 3,0 & 0,92775 & 0,00003 & $-47,61$ & 4,93 & $-7842,63$ & 6,96 & 4998,94 \\
\hline
\end{tabular}


Tabela.7.3. O tempo de geração de nêutrons prontos, $l_{\text {eff }}$, e a fração de nêutrons atrasados, $\beta_{\text {eff }}$ do MCNP6.

\begin{tabular}{|c|c|c|c|c|}
\hline $\begin{array}{c}\text { Posição } \\
\text { dos BC\% }\end{array}$ & $\boldsymbol{l}_{\text {eff }}(\mu \mathrm{seg})$ & $\sigma_{l e f f}(\mu$ seg $)$ & $\boldsymbol{\beta}_{\text {eff }}$ & $\sigma_{\beta e f f}$ \\
\hline 100,0 & 31,68 & 0,00 & 0,00757 & 0,00002 \\
\hline 93,0 & 31,67 & 0,01 & 0,00756 & 0,00002 \\
\hline 90,5 & 31,70 & 0,01 & 0,00755 & 0,00002 \\
\hline 88,0 & 31,71 & 0,01 & 0,00759 & 0,00002 \\
\hline 85,5 & 31,73 & 0,01 & 0,00756 & 0,00002 \\
\hline 83,0 & 31,73 & 0,01 & 0,00757 & 0,00002 \\
\hline 80,5 & 31,76 & 0,01 & 0,00757 & 0,00002 \\
\hline $\mathbf{7 8 , 0}$ & 31,79 & 0,01 & 0,00757 & 0,00002 \\
\hline 75,5 & 31,83 & 0,01 & 0,00757 & 0,00002 \\
\hline 73,0 & 31,86 & 0,01 & 0,00758 & 0,00002 \\
\hline 70,5 & 31,90 & 0,01 & 0,00759 & 0,00002 \\
\hline 68,0 & 31,96 & 0,01 & 0,00761 & 0,00002 \\
\hline 65,5 & 31,99 & 0,01 & 0,00759 & 0,00002 \\
\hline 63,0 & 32,03 & 0,01 & 0,00763 & 0,00002 \\
\hline 60,5 & 32,13 & 0,01 & 0,00758 & 0,00002 \\
\hline $\mathbf{5 8 , 0}$ & 32,16 & 0,01 & 0,00763 & 0,00002 \\
\hline 55,5 & 32,22 & 0,01 & 0,00758 & 0,00002 \\
\hline 53,0 & 32,28 & 0,01 & 0,00757 & 0,00002 \\
\hline 50,5 & 32,34 & 0,01 & 0,00761 & 0,00002 \\
\hline 48,0 & 32,44 & 0,01 & 0,00761 & 0,00002 \\
\hline 45,5 & 32,50 & 0,01 & 0,00761 & 0,00002 \\
\hline 43,0 & 32,56 & 0,01 & 0,00760 & 0,00002 \\
\hline 40,5 & 32,63 & 0,01 & 0,00763 & 0,00002 \\
\hline 38,0 & 32,70 & 0,01 & 0,00758 & 0,00002 \\
\hline 35,5 & 32,76 & 0,01 & 0,00761 & 0,00002 \\
\hline 33,0 & 32,82 & 0,01 & 0,00761 & 0,00002 \\
\hline 30,5 & 32,86 & 0,01 & 0,00760 & 0,00002 \\
\hline 28,0 & 32,93 & 0,01 & 0,00759 & 0,00002 \\
\hline 25,5 & 32,97 & 0,01 & 0,00757 & 0,00002 \\
\hline 23,0 & 33,02 & 0,01 & 0,00761 & 0,00002 \\
\hline 20,5 & 33,03 & 0,01 & 0,00766 & 0,00002 \\
\hline 18,0 & 33,07 & 0,01 & 0,00761 & 0,00002 \\
\hline 15,5 & 33,07 & 0,01 & 0,00763 & 0,00002 \\
\hline 13,0 & 33,07 & 0,01 & 0,00761 & 0,00002 \\
\hline 10,5 & 33,09 & 0,01 & 0,00762 & 0,00002 \\
\hline 8,0 & 33,09 & 0,01 & 0,00761 & 0,00002 \\
\hline 5,5 & 33,07 & 0,01 & 0,00761 & 0,00002 \\
\hline $\mathbf{3 , 0}$ & 33,09 & 0,01 & 0,00758 & 0,00002 \\
\hline
\end{tabular}




\section{ANÁliSE E DISCUSSÃo DOS RESULTADOS TEÓRICOS E EXPERIMENTAIS}

As Fig. 8.1 e Fig. 8.2 mostram, respectivamente, as comparações dos dados teóricos: do GPT-TORT e do MCNP5; com os resultados experimentais dos $\Sigma \rho_{g e n}$ e $\zeta$ da APSD. A condordância entre os dados teóricos e experimentais é bastante boa com os valores da reatividade total $\left(\Sigma \rho_{g e n}\right)$ do GPT-TORT, e muito boa com os do MCNP5. No caso da reatividade total, os resultados dos cálculos do MCNP5 ficam, na maioria dos casos, dentro das barras de erro dos resultados experimentais. Isso reforça a capacidade do método experimental proposto para medir a reatividade subcrítica dos sistemas multiplicativos.

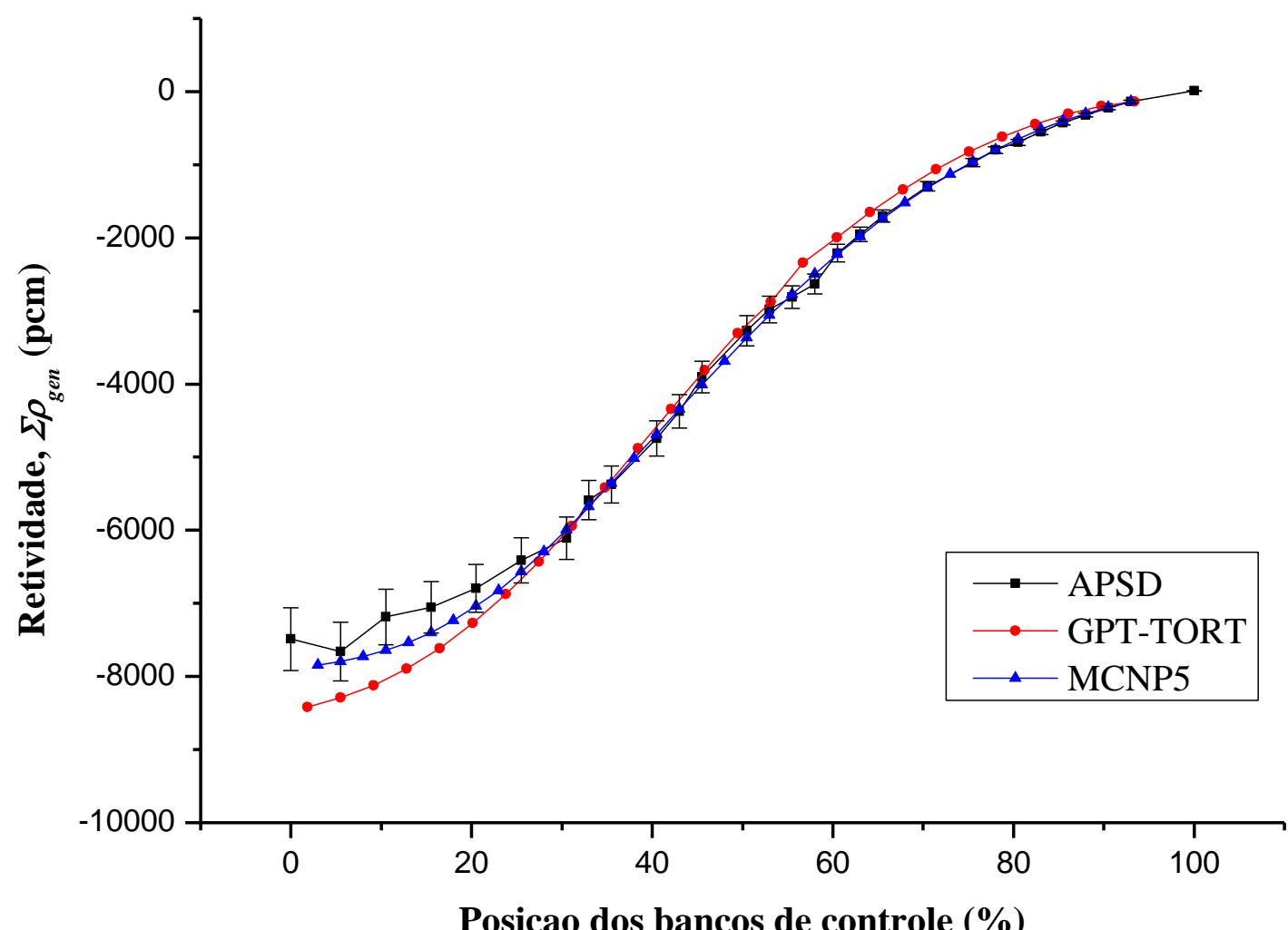

Fig. 8.1. Comparação da reatividade total calculada pelo GPT-TORT(vermelho) e pelo MCNP5(azul) com os resultados experimentais da APSD. 
A concordância dos três modelos para esta grandeza, $\Sigma \rho_{g e n}$, é de boa qualidade, apesar de que os dados do GPT-TORT sejam maiores do que os dos outros até a posição de 55,5\%. A discrepância dos três gráficos começa a revelar-se na posição de $28,0 \%$, e cresce conforme inserem-se mais os bancos de controle. Nota-se que, apesar do aumento da discrepância, os dados do MCNP5 ficam dentro das barras de erros dos dados experimentais. A mesma figura mostra, também, que a sensibilidade do modelo do GPT-TORT à inserção dos bancos de controle é menor, entre os três modelos, na região próxima da criticalidade, porém maior na região mais subcrítica.

A comparação teoria/experimento para o índice de subcriticalidade $(\zeta)$, mostrada na Fig. 8.2, revela discrepância considerável.

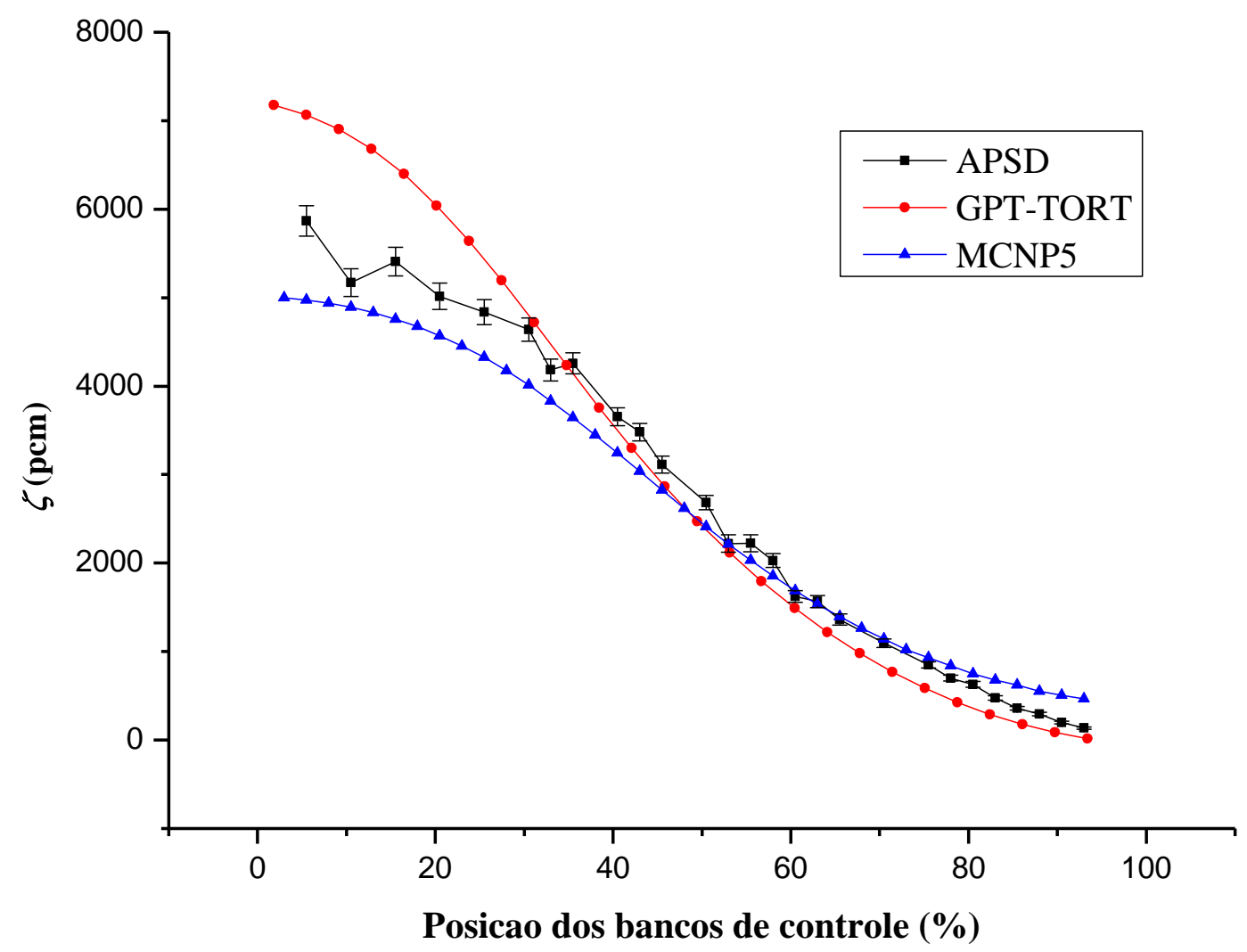

Fig. 8.2. Comparação do índice de subcriticalidade ( $\zeta$ ) calculada pelo TORT(vermelho) e pelo MCNP5(azul) com os resultados experimentais da APSD. 
Nesse caso, pouco mais de trabalho seria necessário para modelar a fonte de Am-Be no refletor. $\mathrm{O}$ índice de subcriticalidade é fortemente dependente da posição da fonte e de suas características geométricas e materiais. No modelo do GPT-TORT, a fonte de Am-Be foi confinada dentro de um único mesh que é muito menor do que seu tamanho real. Além disso, outros materiais da fonte como o cilíndro acrílico, por exemplo, não foram modelados adequadamente e foram simplesmente substituídos por água do refletor.

Além do mais, os dados do MCNP5 e do GPT-TORT foram obtidos levandose em conta uma fonte adicional de Am-Be de 1Ci na lateral do núcleo em todos os casos. Não obstante, no experimento da APSD houve variação da intensidade da fonte, como pode ser visto na Tabela 3.1.

Para uma comparação melhor dos resultados, Fig. 8.3 mostra os desvios relativos dos valores calculados, dados por: $(C-E) / E$, onde $C$ e $E$ representam os resultados calculados e experimentais, respectivamente. Aqui, alguns detalhes da comparação teoria/experimento tornam-se mais claras. Pode ser visto e, também quantificado, que as reatividades totais do MCNP5 concordam melhor com os valores experimentais do que as do GPT-TORT. Além disso, como já foi dito, os resultados dos cálculos do MCNP5 ficam, na maioria dos casos, dentro das barras de erro dos dados experimentais. As maiores discrepâncias e os maiores erros ocorrem quando os bancos de controle aproximam-se da posição da máxima retirada, porque nesta região a reatividade total é pequena, enquanto sua incerteza experimental é grande. A discrepância diminui à medida que os bancos de controle são inseridos, a despeito de que a discrepância dos próprios valores da $\Sigma \rho_{\text {gen }}$ seja maior nesta região. 


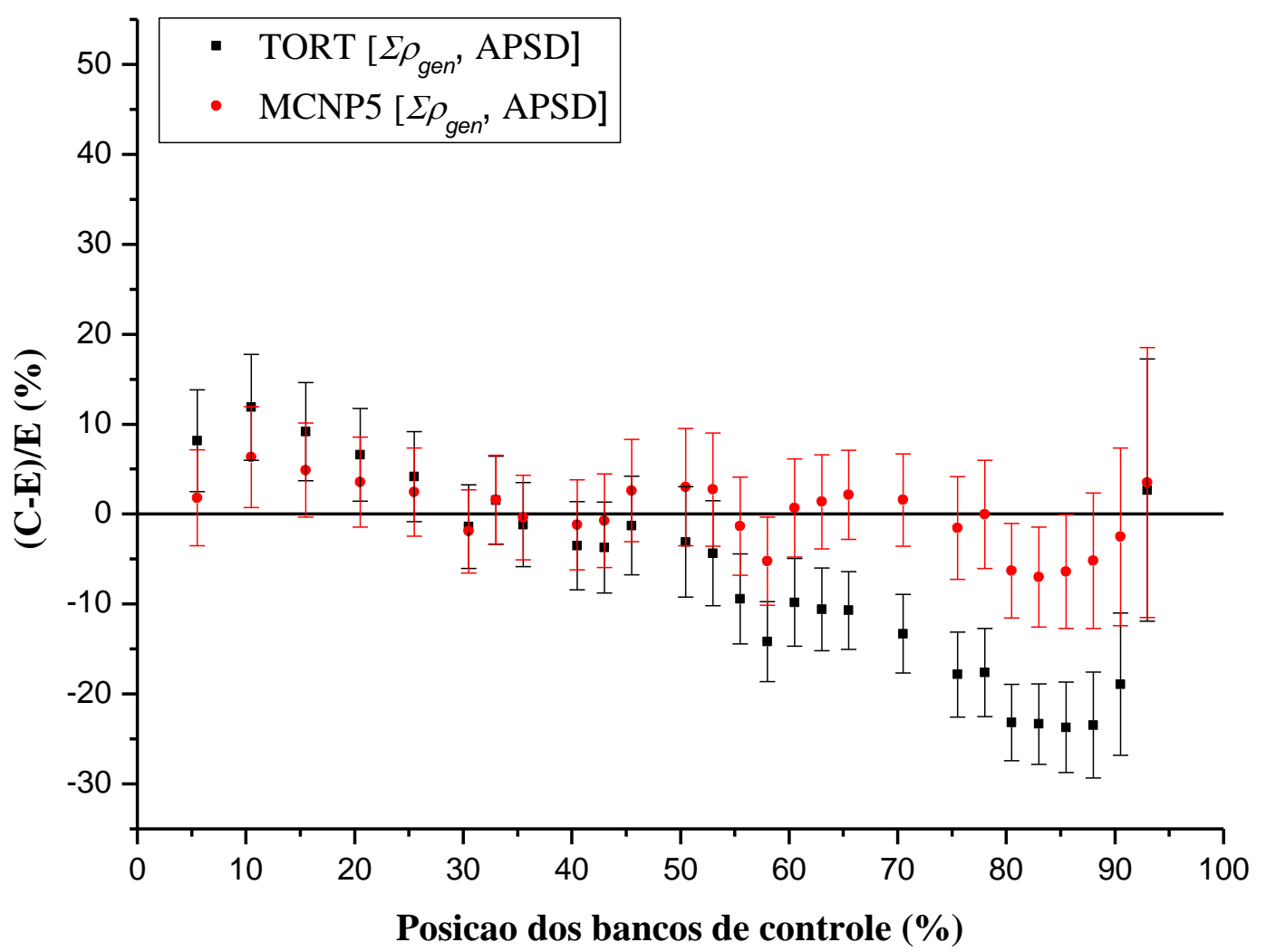

Fig. 8.3. Desvio relativo entre valores calculados $(C)$, pelo TORT (cor preta) e pelo MCNP5 (cor vermelha), e os experimentais $(E)$ da reatividade generalizada total $\left(\Sigma \rho_{\text {gen }}\right)$ do experimento da APSD.

O desvio relativo entre os valores calculados e experimentais do índice de

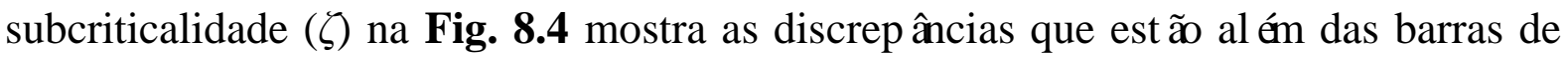
erro. A mesma figura mostra, também, que, no caso do GPT-TORT, essa grandeza tem tendência de ser subestimada à medida que os bancos de controle se aproxime da posição da máxima remoção dos bancos e, de ser superestimada, no sentido oposto. Em adição, no caso do MCNP5, a discrepância é menor e mais uniforme na maioria dos casos; porém, observa-se uma tendência de o parâmetro ser superestimado quando o sistema tende à criticalidade. 


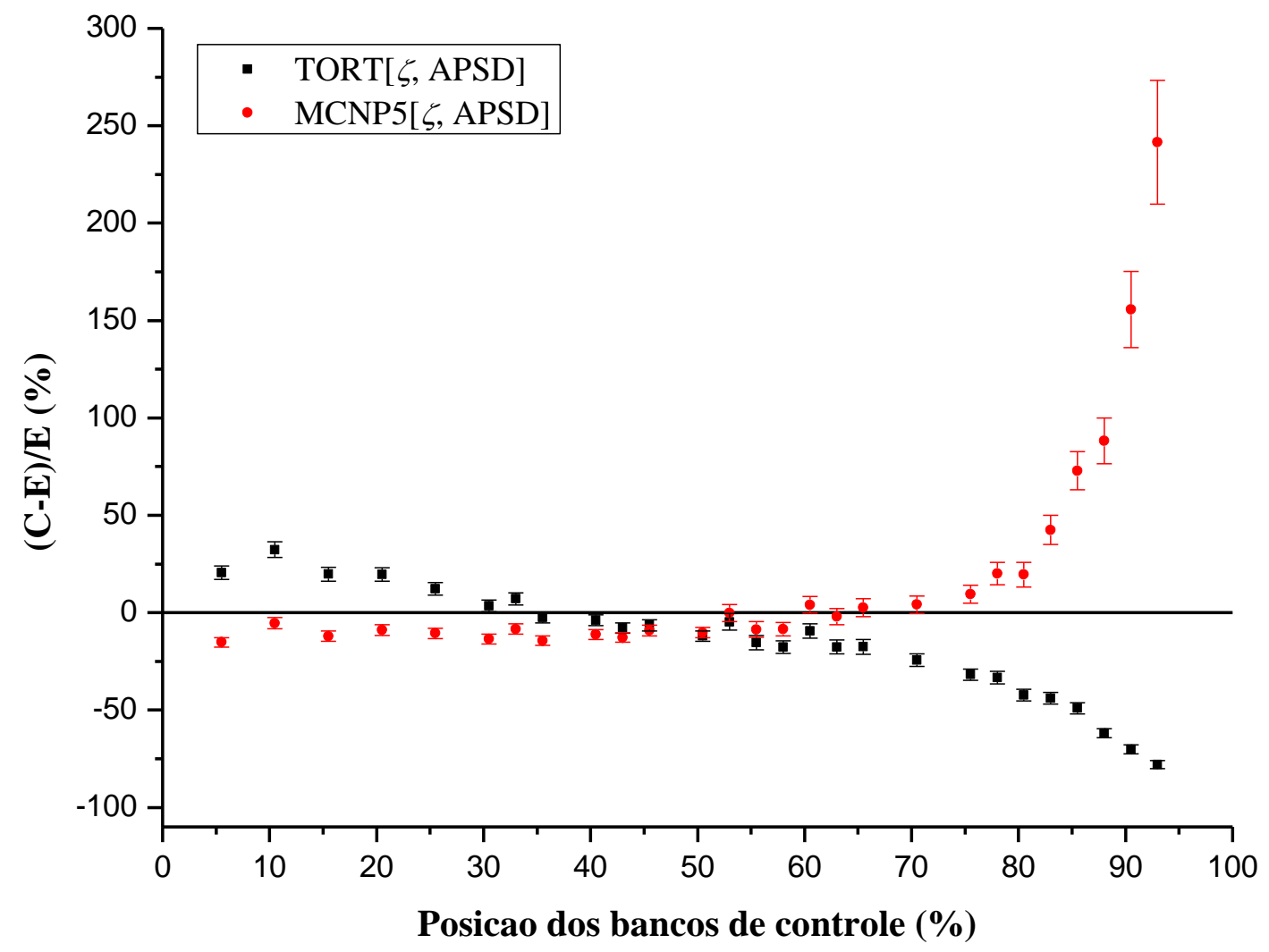

Fig. 8.4. Desvio relativo entre valores calculados $(C)$ e experimentais $(E)$ do índice de subcriticalidade $(\zeta)$ do experimento da APSD.

Como mencionado na seção anterior, as posições dos resultados do GPTTORT são diferentes aos do experimento e do MCNP5. Por isso, foi necessário efetuar interpolações dos resultados do GPT-TORT nas posições dos experimentos, por meio de ajuste de uma curva da função, polinomial, aos pontos nos gráficos dos dados calculados. As funções de ajuste com os respectivos coeficientes e seus gráficos, e os valores de $\Sigma \rho_{g e n}$ de $\zeta$ interpolados encontram-se em APÊNDICE E.

Foram feitas as mesmas comparações com os resultados da CPSD. A Fig. 8.5 e a Fig. 8.6 mostram as reatividades $\left(\Sigma \rho_{\text {gen }}\right)$ e o índice de subcriticalidade $(\zeta)$, respectivamente. A Fig. 8.5 mostra, mais uma vez, que as $\Sigma \rho_{\text {gen }}$ experimentais da CPSD concordam melhor com os resultados do MCNP5, como no caso da APSD. 


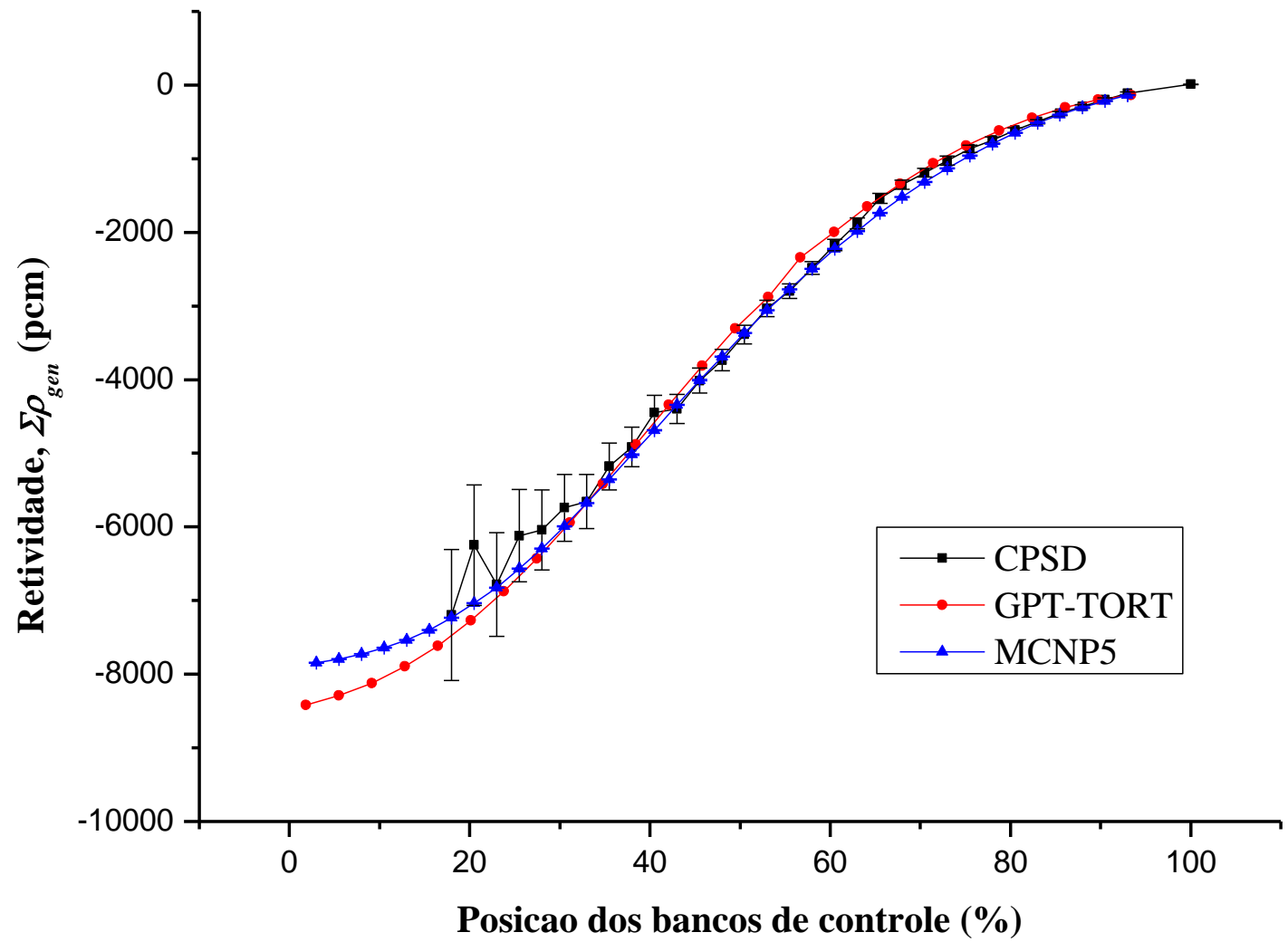

Fig. 8.5. Comparação da reatividade total calculado pelo GPT-TORT(vermelho) e pelo MCNP5(azul) com os resultados experimentais da CPSD.

A qualidade dos dados experimentais da CPSD foi inferior à dos resultados da APSD, até o ponto que o experimento da CPSD foi interrompido na posição de $18 \%$ e não se prosseguiu mais. A flutuação dos dados da reatividade total é observada logo a partir da posição 23\%. A situação é mesma ou ainda pior com os dados de duas APSDs (APSD1 e APSD2) ligadas com a CPSD, conforme ilustrado na Fig. 5.3.

Os detectores utilizado nos dois experimentos foram os mesmos; na região mais subcrítica onde ocorreu a flutuação na CPSD foram usados o detector de ${ }^{3} \mathrm{He}$ de sensibilidade alta para APSD1 e o detector ${ }^{3} \mathrm{He}$ de sensibilidade média para APSD2, como mostrado na Tabela 3.2. No caso da APSD, o detector de ${ }^{3} \mathrm{He}$ de sensibilidade alta foi utilizado nessa região de subcriticalidade.

O que mudou no arranjo experimental, da APSD para CPSD(APSD1 e APSD2) 
foi a posição dos detectores. Conforme ilustrado em Fig. 3.7 e Fig. 3.11., o detector foi colocado no meio da face oeste do núcleo no experimento da APSD, ou seja, a posição é mais próxima da região ativa do núcleo do que as duas posições de detectores do experimento da CPSD. Além disso, com presença de materiais absorvedores das barras de controle, o núcleo é mais assimétrico para os dois detectores do experimento da CPSD, pois os detectores estão posicionados na mesma face (oeste), porém os bancos de controle são arranjados em diagonal. Ou seja, um dos detectores ficou mais próximo das barras de controle do que outro, e isso deve comprometer a qualidade da medida da CPSD, que por sua vez é produto de correlação dos sinais que provem dos dois detectores.

O efeito dessa desigualdade para os detectores no experimento da CPSD parece ser atenuado na região próxima da criticalidade devido ao grande número de nêutrons no sistema, mas vem a ser cada vez mais evidente à proporção que o sistema torna-se mais subcrítico.

Além disso, o índice de subcriticalidade, $\zeta$, é mais afetado pela desigualdade dos detectores da CPSD, como mostrado em Fig. 8.6. 


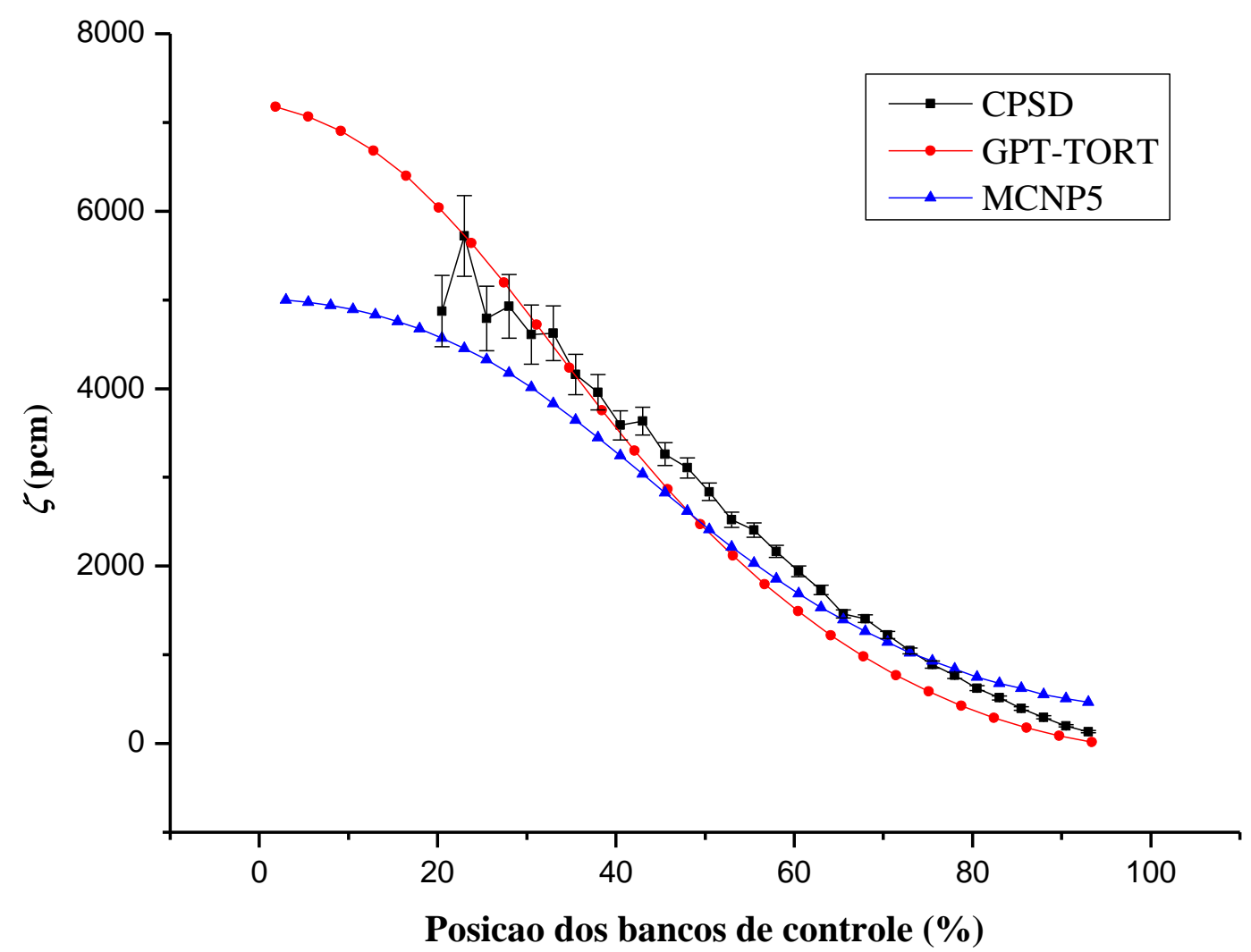

Fig. 8.6. Comparação do índice de subcriticalidade ( $\zeta$ calculada pelo TORT(vermelho) e pelo MCNP5(azul) com os resultados experimentais da CPSD.

Em comparação com os resultados da APSD, a flutuação do $\zeta$ experimental é mais acentuada e a discrepância entre os resultados teóricos e experimentais é maior. Isso mostra, mais uma vez, que o índice de subcriticalidade é uma grandeza muito sensível à posição do detector, também. $\mathrm{O}$ aumento da discrepância, em comparação com a APSD, fica mais evidente à medida que os bancos de controle se aproximem do centro do núcleo.

A Fig. 8.7 mostra o desvio relativo das reatividades totais teóricos em relação às experimentais, onde se pode observar que a superioridade do MCNP5 em termos de concordância com os dados experimentais diminuiu-se significativamente em relação ao caso da APSD. 


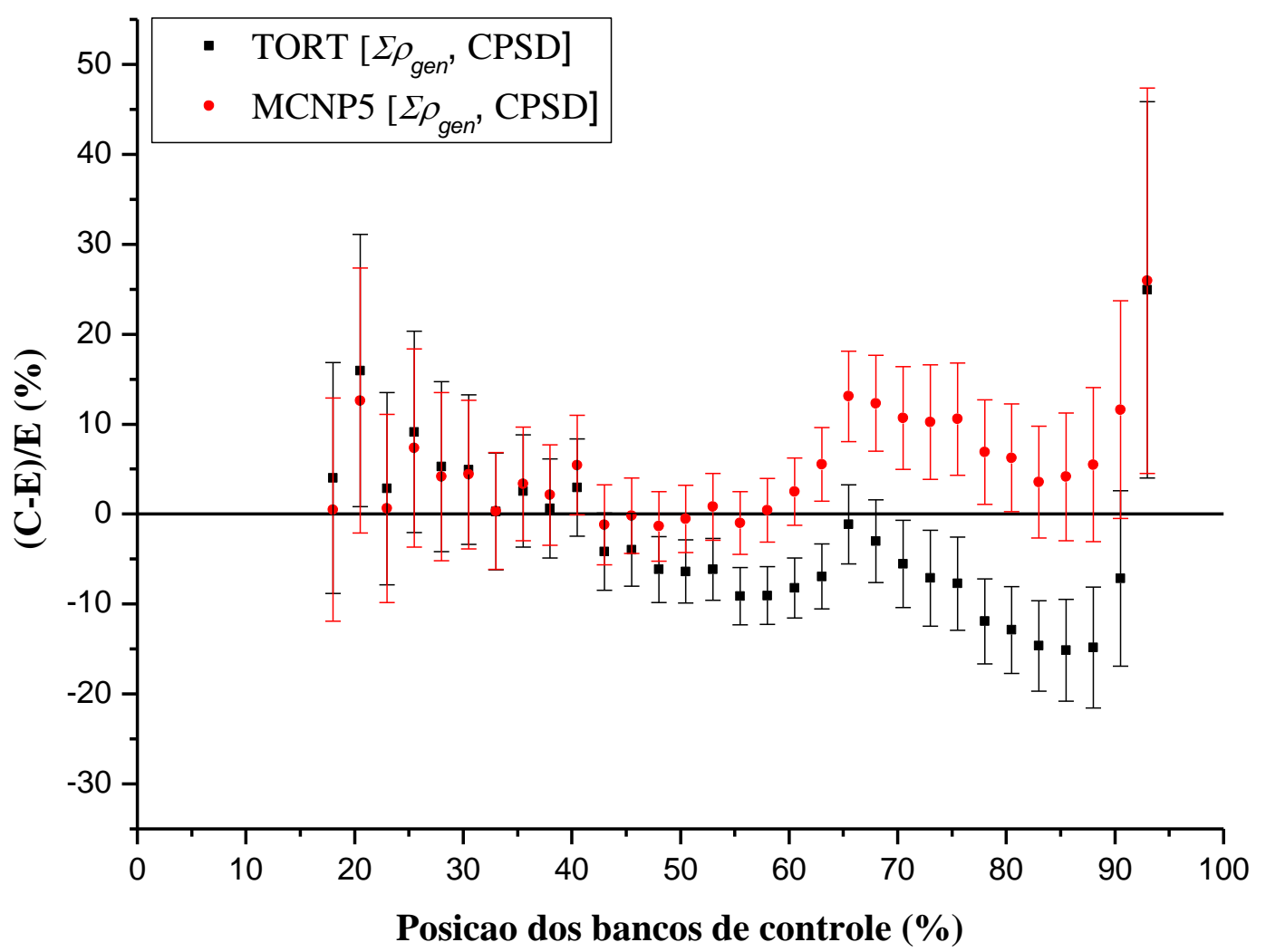

Fig. 8.7. Desvio relativo entre valores calculados $(C)$, pelo TORT (cor preta) e pelo MCNP5 (cor vermelha), e os experimentais $(E)$ da reatividade generalizada total $\left(\Sigma \rho_{g e n}\right)$ do experimento da CPSD.

Neste gráfico, nota-se claramente mudança brusca no comportamento dos dados, tanto do MCNP5 como do GPT-TORT, na posição de 63\%, na qual foi posicionada a fonte adicional de $\mathrm{Am}-\mathrm{Be}$ de $1 \mathrm{Ci}$ na face leste do núcleo, conforme mostrado na Fig. 3.11. Esse fenômeno não foi observado no caso da APSD.

Os desvios relativos do $\zeta$ do caso da CPSD são mostrados na Fig. 8.8, onde nota-se que os dados do MCNP5 concordam melhor com os experimentais, na maioria dos casos, que os dados do GPT-TORT. Tanto a tendência do modelo do MCNP5 de superestimar o $\zeta$,como a propensão do modelo do GPT-TORT de subestimá-lo quando o sistema tende à criticalidade são observadas na figura, também, como no caso da APSD. 


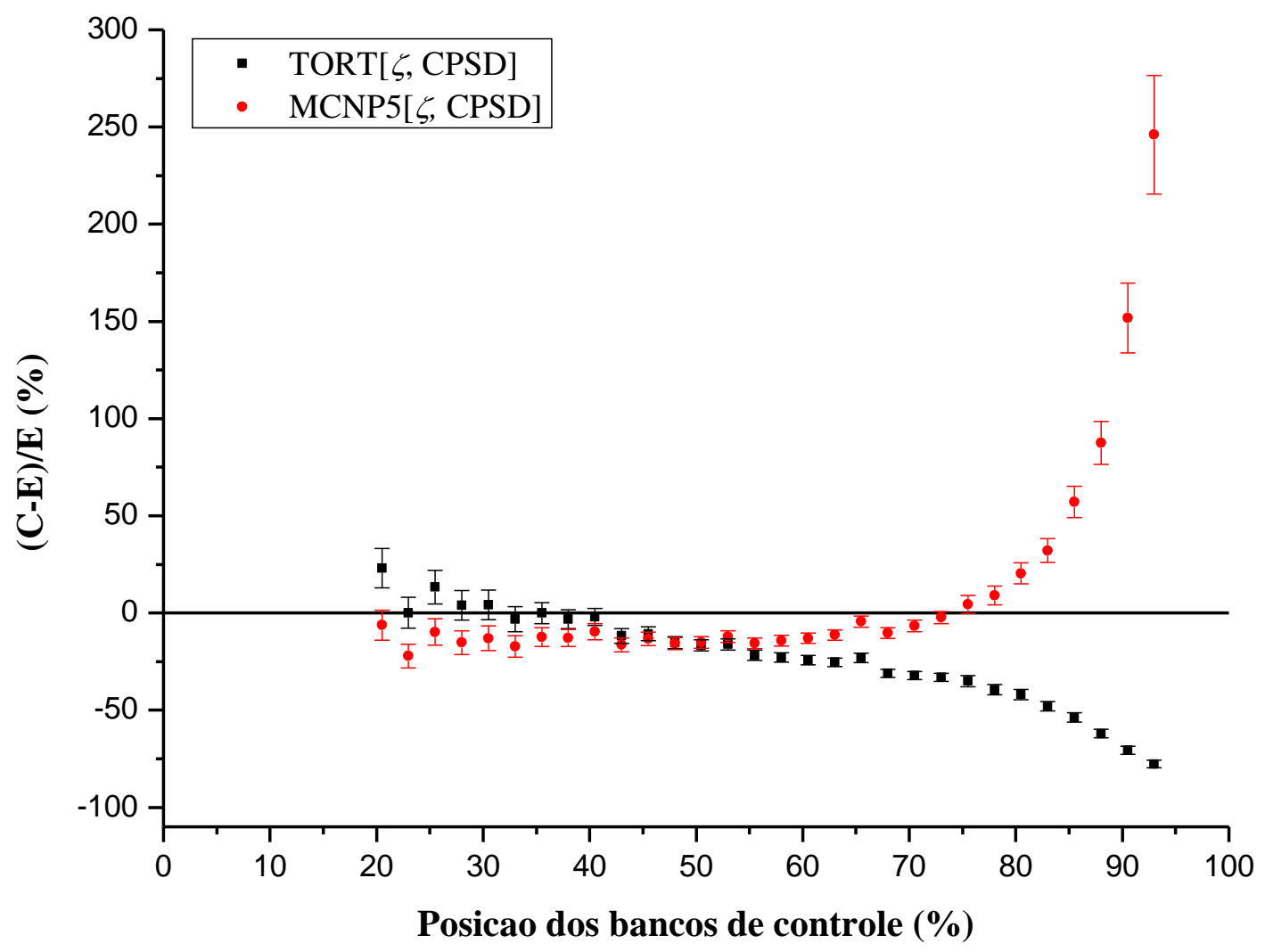

Fig. 8.8. Desvio relativo entre valores calculados $(C)$ e experimentais $(E)$ do índice de subcriticalidade $(\zeta)$ do experimento da CPSD.

Observa-se, também, na Fig. 8.7 e Fig. 8.8 que, os dados experimentais começam a perder confiabilidade a partir da posição de $40,5 \%$, visto que as incertezas aumentam significativamente no sentido da inserção dos bancos de controle, além de que os valores flutuarem de maneira irregular. Por esta razão os resultados experimentais das posições mais subcríticas foram ignorados na seção $\mathbf{5 . 2}$.

Outra grandeza pela qual os três modelos podem ser comparados é o fator de multiplicação efetivo, $k_{\text {eff. }}$ A Fig. 8.9. e Fig. 8.10 mostram os $k_{\text {eff }}$ experimentais da APSD e da CPSD, respectivamente, calculados pela Eq. (5.5) junto com os valores calculados pelo modelo do GPT-TORT e do MCNP5. 


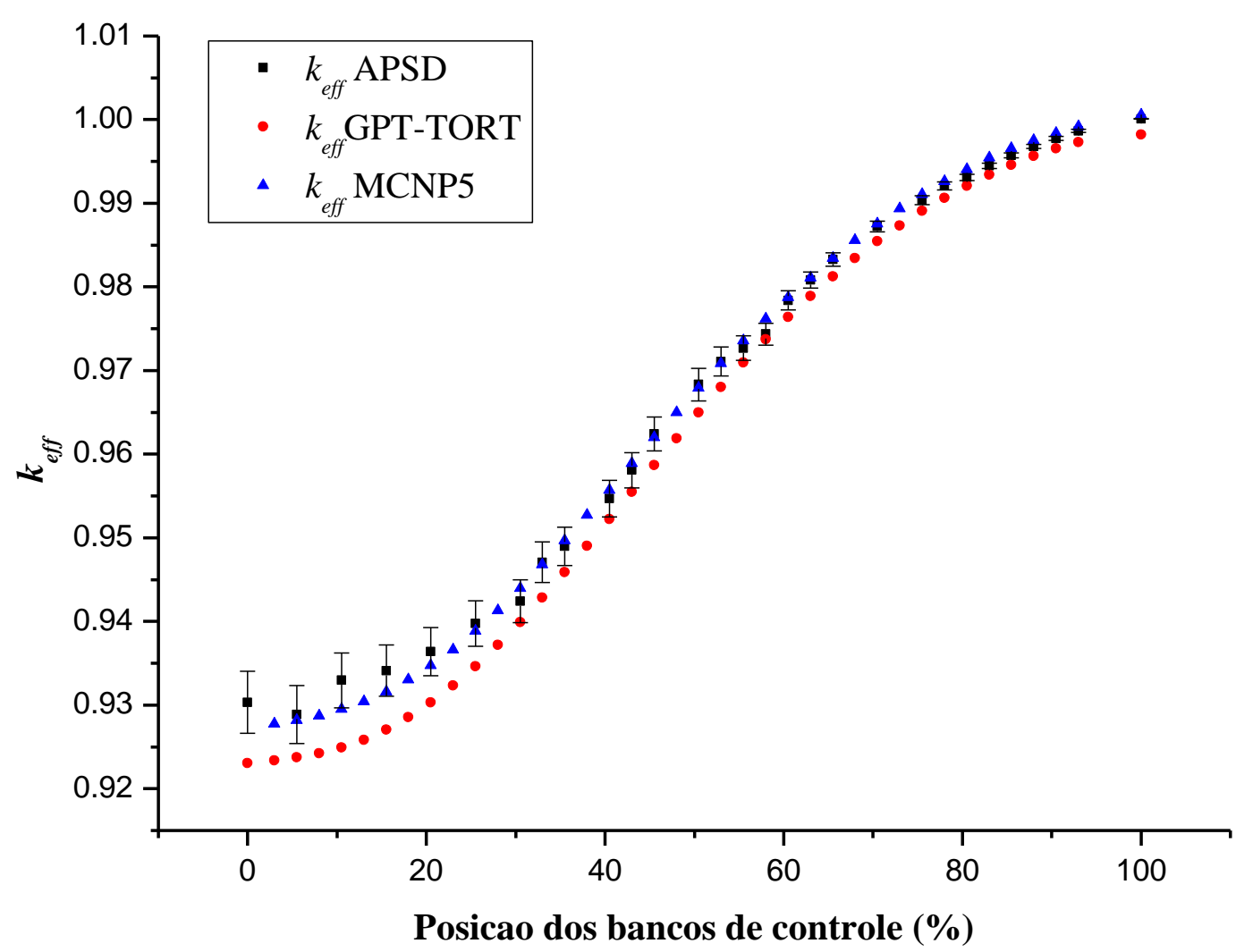

Fig. 8.9. Os $k_{e f f}$ da APSD em comparação com os valores calculados do GPT-TORT(cor vermelha) e MCNP5(cor azul)

É bom lembrar que a reatividade total $\left(\Sigma \rho_{g e n}\right)$ do MCNP5 foi calculado a partir do valor do $k_{\text {eff }}$ de saída da simulação, pela Eq. (6.1). No entanto, no GPT-TORT, o arquivo de entrada para o cálculo do $k_{\text {eff }}$ foi independente do arquivo para o cálculo de outras grandezas como $\rho_{\text {gen }}$, por exemplo; embora os dois arquivos de entrada compartilhassem a mesma configuração geométrica.

Como é de esperar, os dados do MCNP5 ficam, na maioria dos casos, dentro de barras de erros dos dados experimentais, tanto da APSD como da CPSD. Por outro lado, a maioria dos dados do GPT-TORT, em ambos os casos, fica fora dessas barras. 


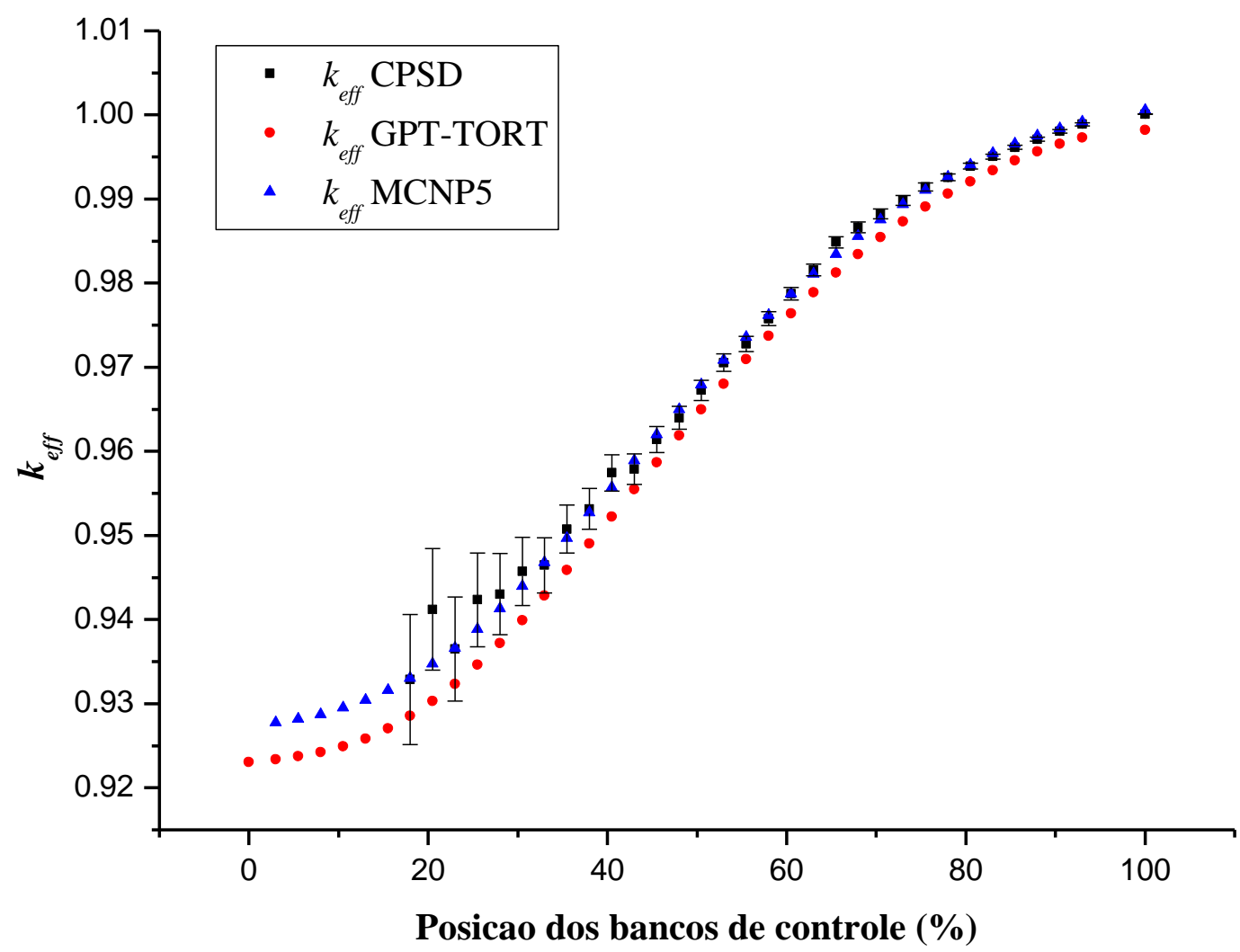

Fig. 8.10. Os $k_{\text {eff }}$ da CPSD em comparação com os valores calculados do GPT-TORT(cor vermelha) e MCNP5(cor azul)

Contudo, uma comparação melhor pode ser feita pelo desvio relativo, i.e., (CE)/E, conforme mostrado na Fig. 8.11. Na figura fica claro que a concordância dos três resultados é muito boa nos dois casos, embora o MCNP5 concorde sempre melhor com os experimentais. Observe que a escala do eixo da ordenadas abrange de $-2,0 \%$ a $1,0 \%$. Isso reforça, ainda mais, a capacidade do método experimental para a medida da reatividade subcrítica dos sistemas multiplicativos. 


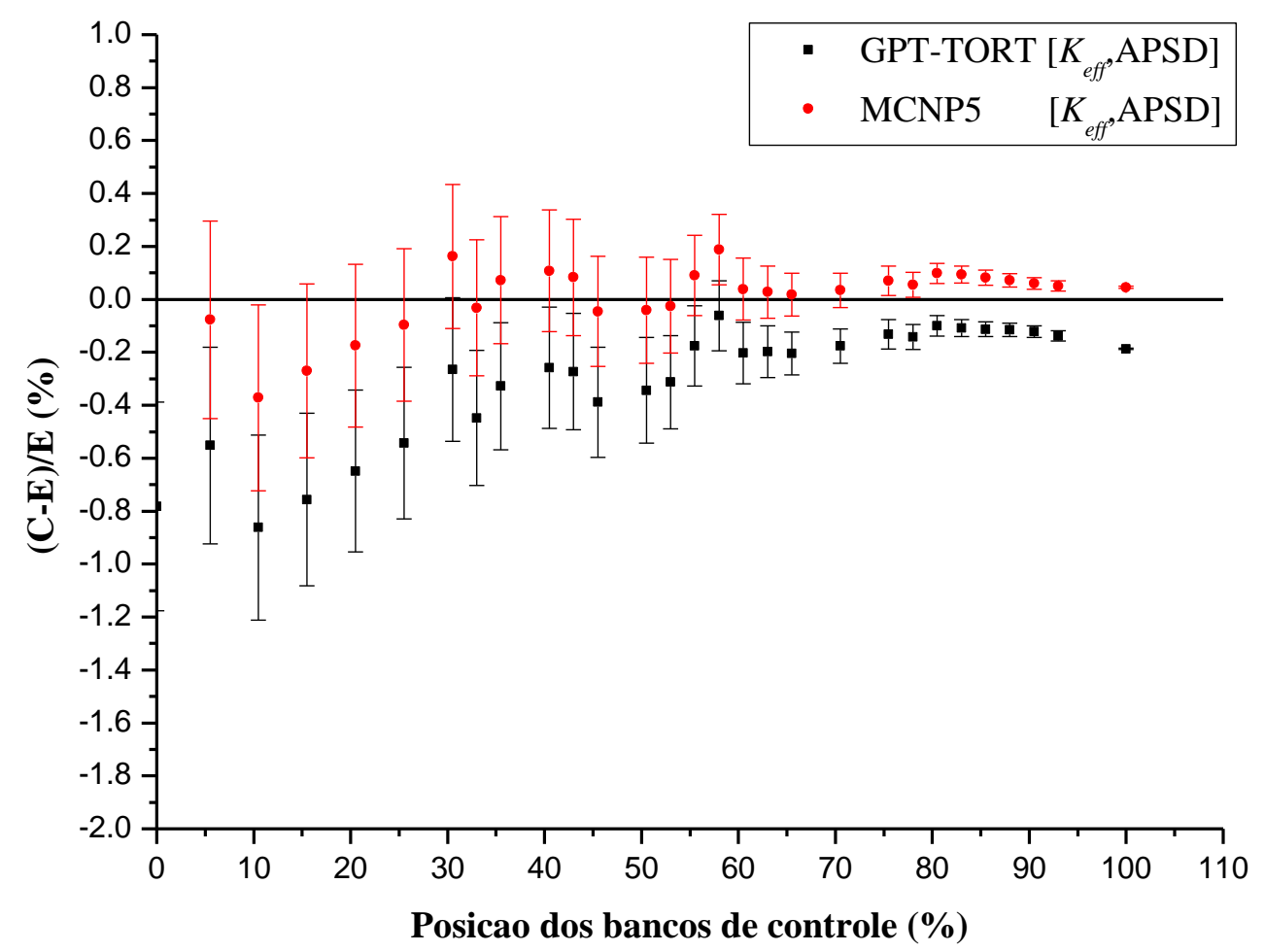

(a)

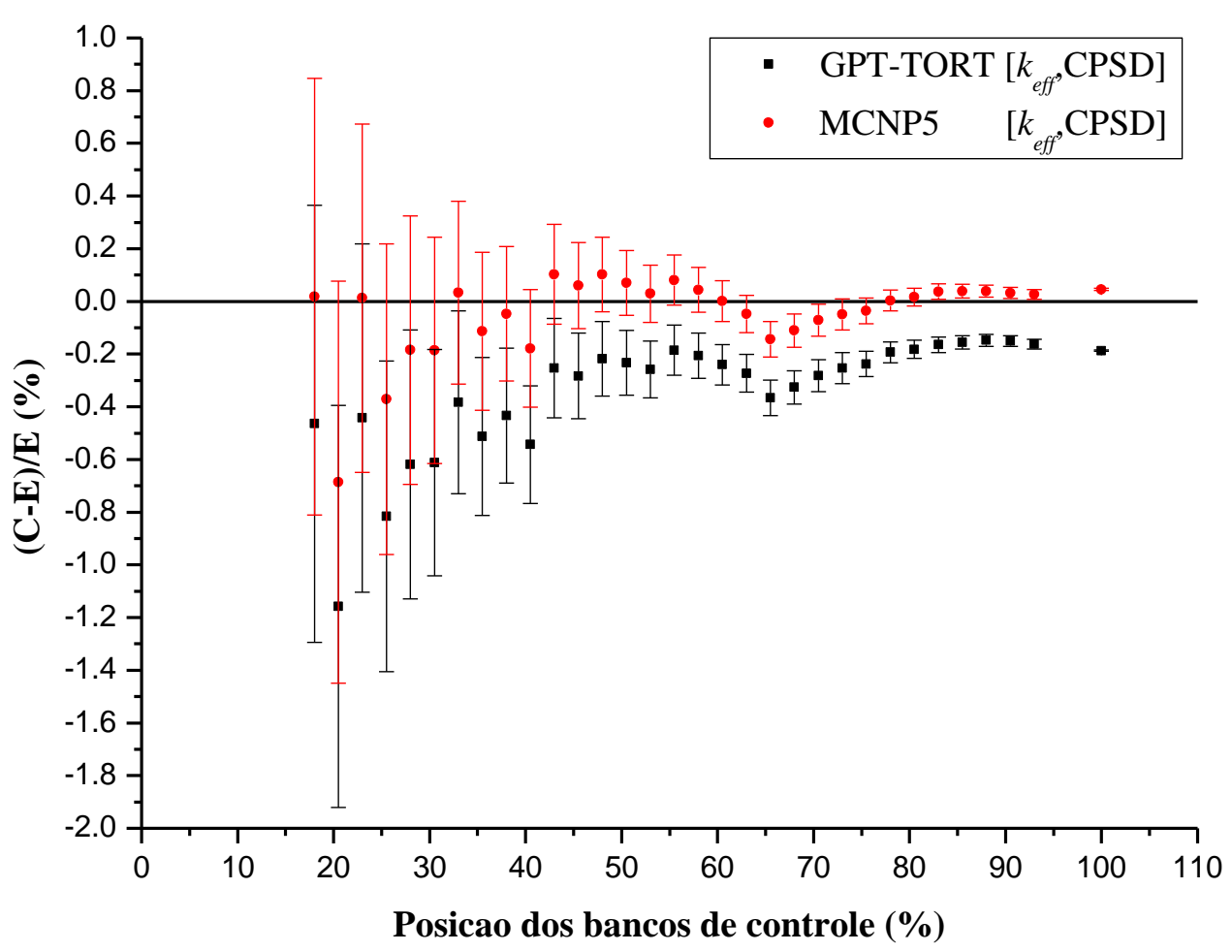

(b)

Fig. 8.11. Desvio relativo entre valores calculados $(C)$, pelo TORT(cor preta) e pelo MCNP5(cor vermelha), e os dados experimentais $(E)$ do fator de multiplicação efetiva $\left(k_{e f f}\right)$ do experimento (a) da APSD e (b) da CPSD. 
Por último, como foi mencionado já no resumo do trabalho, o tempo de geração de nêutrons prontos, $l_{\text {eff }}$, e a fração de nêutrons atrasados, $\beta_{\text {eff }}$, foram assumidos constantes no tratamento de dados experimentais. Contudo, essas grandezas calculadas pelo modelo do GPT-TORT e pelo MCNP5 variam com o grau da subcriticalidade do sistema, conforme mostrado na Tabela. 7.1 e Tabela. 7.3, respectivamente.

A Fig. 8.12 mostra as variações dos $l_{\text {eff }}$ e $\beta_{\text {eff }}$ dos três métodos em relação ao índice de subcriticalidade $(\zeta)$. Na figura, pode-se notar que o tempo de geração de nêutrons prontos dos métodos teóricos tem comportamentos lineares em função de $\zeta$, e aumentam à medida que o sistema torna-se cada vez mais subcrítico. Esses comportamentos são esperados, pois, quanto mais subcrítico o sistema tanto maior o tempo de vida de nêutrons. Fica claro que os $l_{\text {eff }}$ teóricos são maiores que o valor assumido no experimento em estados subcríticos, e que o modelo do GPT-TORT, mais uma vez, mostrou-se mais sensível ao aumento do grau de subcriticalidade.

Quanto à fração de nêutrons atrasados, os resultados teóricos dos ambos modelos não mostram variações sistemáticas em relação ao $\zeta$,embora os dados do MCNP5 tem flutuações em torno de um certo valor da $\beta_{\text {eff. }}$. 


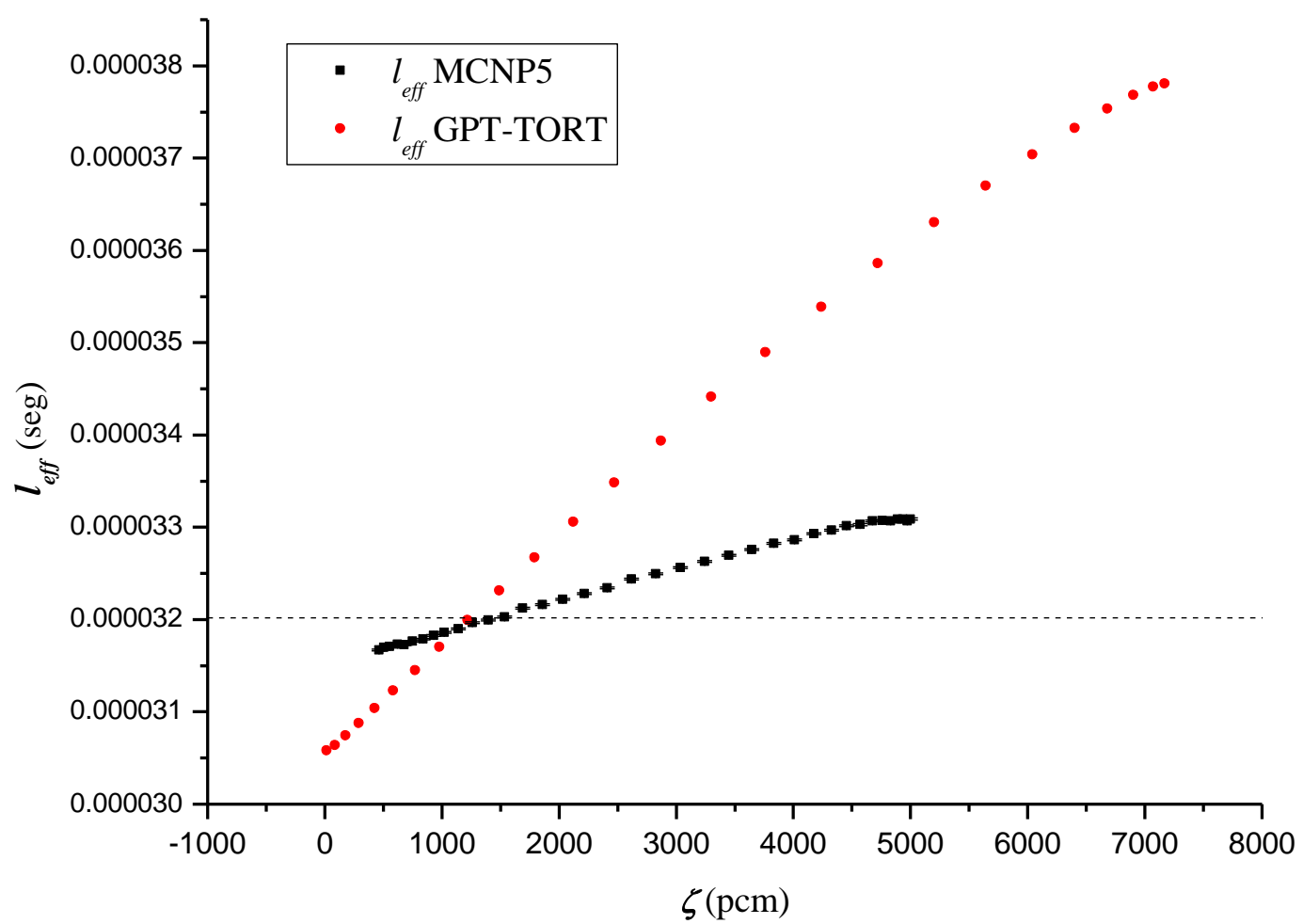

(a)

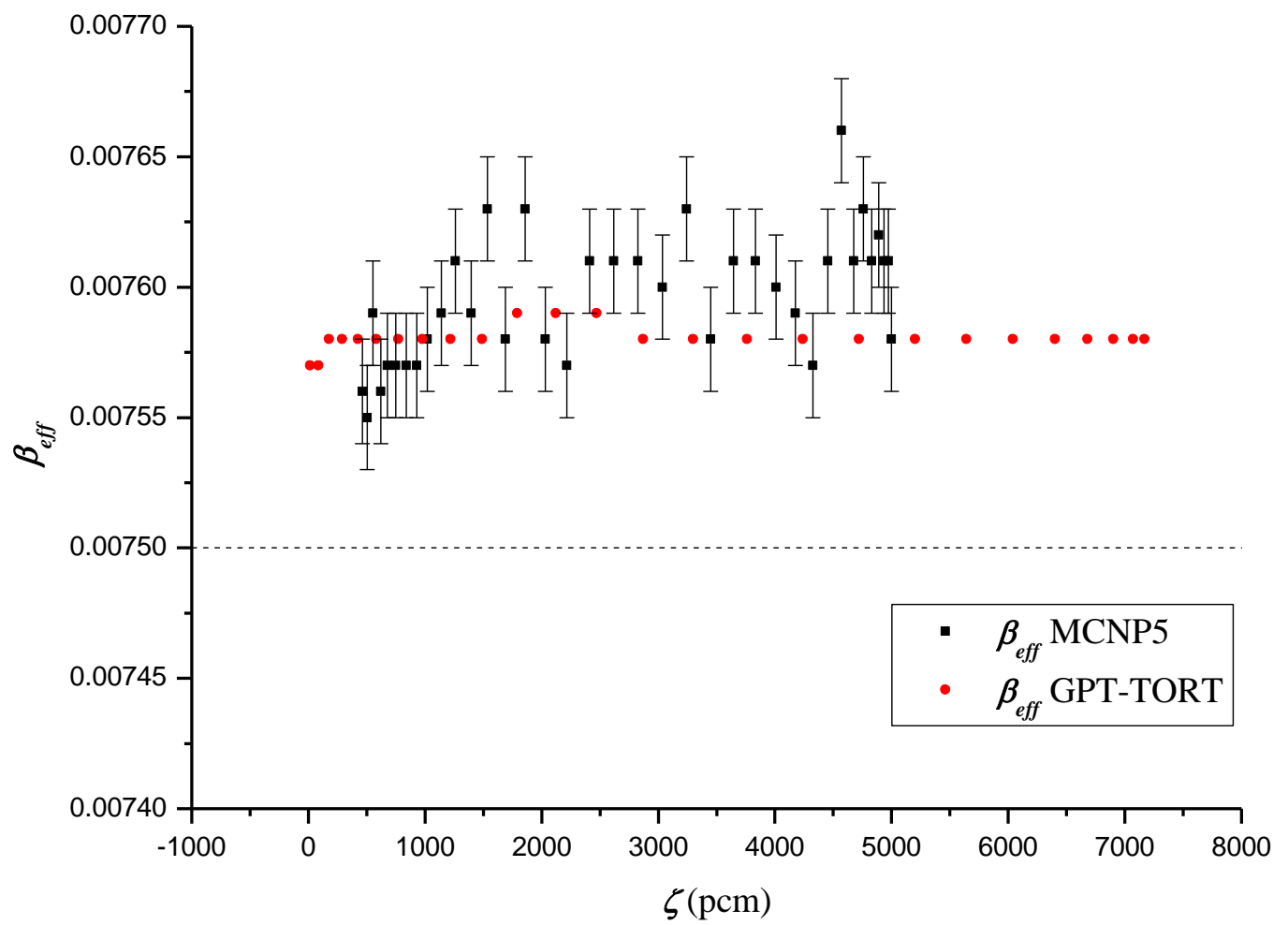

(b)

Fig. 8.12 Os valores (a) do tempo de geração de nêutrons prontos, $l_{\text {eff }}$; (b) da fração efetiva de nêutrons atrasados, $\beta_{\text {eff }}$ do GPT-TORT(cor vermelha) e do MCNP(cor preta). Os valores utilizados para tratamentos de dados experimentais estão indicados pelas linhas pontilhadas. 


\section{CONCLUSÃO}

O método experimental proposto para a determinação da reatividade subcrítica tem se mostrado ser viável e apropriado para lidar com sistemas subcríticos. Os experimentos em diversas configurações subcríticas do IPEN/MB-01 foram realizados com sucesso e, foram obtidos dados de boa qualidade. Como mencionado previamente, o método proposto baseia-se apenas em grandezas medidas tais como a taxa de contagem e os parâmetros decorrentes do ajuste dos mínimos quadrados da APSD e da CPSD. Outras grandezas complicadas, frequentemente usadas em vários outros métodos, tais como a eficiência do detector, não são necessárias neste método. Esta abordagem experimental pode ser considerada muito útil para a determinação e descrição dos níveis subcríticos em sistemas do reator, e forneceu resultados muito bons e encorajadores. Foi demonstrado que o modelo da cinética subcrítica desenvolvido por Gandini e Salvatores descreve a física envolvida nos sistemas multiplicativos subcríticos. Os resultados experimentais mostraram claramente que a teoria da cinética pontual clássica não é capaz de descrever a reatividade medida. As comparações de teoria/experimento revelam que a concordância das reatividades totais obtidas pelo MCNP5 com os valores experimentais é muito boa. O GPT-TORT também produziu bons resultados. Uma vez que ambas metodologias utilizam ENDF/B-VII.0 como base de dados nucleares, pode-se afirmar que esta biblioteca teve um bom desempenho na comparação de teoria/experimento. Além disso, foi mostrado que o índice de subcriticalidade $(\zeta)$ obtido pelo GPT-TORT apresenta algumas discrepâncias; e seus valores calculados têm uma tendência de ser subestimados perto do estado crítico, e superestimado, em estados mais subcríticos. A modelagem da fonte, junto com a sua estrutura interna, pode ser importante para melhorar a comparação de teoria/experimento do índice de subcriticalidade (५). Além disso, a posição de detector parece influenciar significativamente na qualidade dos dados experimentais, principalmente do índice de subcriticalidade. 


\subsection{Trabalhos Futuros}

Uma sugestão para trabalhos futuros é reproduzir o experimento apresentado neste trabalho com maior número de médias, $N$, nas aquisições de dados com finalidade de melhorar a estatística de dados, e assim, obter melhores resultados experimentais. O número de médias deste trabalho varia entre 500 e 1000. Porém, foi constatado num experimento relacionado com este que, aumentando o número de média para 3000, a estatística de APSD melhora significativamente, já nas regiões perto da criticalidade. Portanto, espera-se que as flutuações dos resultados finais nas regiões mais subcríticas observadas neste trabalho sejam atenuadas com o $N$ igual ou maior que 3000 .

Um trabalho de doutorado relacionado a este está em andamento, por Eduardo Gonelli, no qual obtêm as densidades espectrais variando o número de varetas de combustíveis do núcleo, mantendo sempre as barras de controle e as de segurança na posição de máxima remoção. O núcleo torna-se cada vez mais subcrítico à medida que as varetas são retiras. Desta maneira, espera-se que o efeito de sombreamento causado pela presença de materiais absorvedores das barras na região ativa do núcleo seja minimizado, ou até eliminado. Outra expectativa deste método é que o sistema mantenha, até certo grau, a simetria em virtude de "não-inseção" das barras de controle.

Outro trabalho previsto para dar início ainda neste ano, consiste em variação da concentração do boro na água do moderador do núcleo. Neste caso, a subcriticalidade do núcleo será controlada somente pela concentração, com as barras de controle e de segurança totalmente retiradas e mantendo os outros componentes do núcleo inalterados. Desta forma, espera-se que o sistema seja mantido mais simétrico possível durante todo o experimento. 


\section{APÊDICE A}

Dedução da Função Transferência, $G(f)$, a partir das Equações de Cinética de Gandini e Salvatores.

Considere as Eq. (2.10) e Eq. (2.11):

$l_{\text {eff }} \frac{d P_{N}(t)}{d t}=\left(\rho_{\text {gen }}-\alpha_{p d} \beta_{e f f}\right) P_{N}(t)+\alpha_{p d} \sum_{i=1}^{I} \lambda_{i} \xi_{i}(t)+\zeta\left(1-P_{N}(t)\right)+\rho_{\text {source }}$

e

$\frac{d \xi_{i}(t)}{d t}=\beta_{e f f, i} P_{N}(t)-\lambda_{i} \xi_{i}(t)$.

A partir da A.2 infere-se o a seguinte:

$\frac{d}{d t} \sum_{i=1}^{I} \xi_{j}(t)=P_{N} \sum_{i=1}^{I} \beta_{e f f, i}(t)-\sum_{i=1}^{I} \lambda_{i} \xi_{j}(t)=\beta_{e f f} P_{N}-\sum_{i=1}^{I} \beta_{e f f, i}(t)$.

Agora, somam-se A.1 e A.3:

$$
\begin{aligned}
\frac{d}{d t}\left(P_{N}(t)+\sum_{i=1}^{I} \xi_{i}(t)\right)= & \left.\frac{\rho_{g e n}-\alpha_{p d} \beta_{e f f}-\zeta}{l_{e f f}}+\beta_{\text {eff }}\right) P_{N}(t)+\left(\frac{\alpha_{p d}}{l_{e f f}}-1\right) \sum_{i=1}^{I} \lambda_{i} \xi_{i}(t) \\
& +\left(\frac{\zeta+\rho_{\text {source }}}{l_{\text {eff }}}\right)
\end{aligned}
$$

Obviamente, no estado estacionário a A.4 torna-se em: 


$$
\left(\frac{\rho_{\text {gen }, o}-\alpha_{p d} \beta_{e f f}-\zeta}{l_{e f f}}+\beta_{e f f}\right) P_{N, o}+\left(\frac{\alpha_{p d}}{l_{e f f}}-1\right) \sum_{i=1}^{I} \lambda_{i i} \xi_{i, o}+\left(\frac{\zeta+\rho_{\text {source }, o}}{l_{\text {eff }}}\right)=0 .
$$

Observe os subíndices onde aparece " $o$ " que indica que os parâmetros correspondentes são do estado estacionário ou não-perturbado.

Agora, suponha que o sistema inicialmente em estado estacionário sofre as seguintes perturbações:

$$
\begin{aligned}
& \rho_{\text {gen }, o} \rightarrow \rho_{\text {gen }, o}+\delta \rho_{\text {gen }}, \\
& \rho_{\text {source }, o} \rightarrow \rho_{\text {source }, o}+\delta \rho_{\text {source }} .
\end{aligned}
$$

Essas perturbações resultam:

$$
\begin{aligned}
& P_{N, o} \rightarrow P_{N, o}+\delta P_{N}, \\
& \xi_{i, o} \rightarrow \xi_{i, o}+\delta \xi_{i} .
\end{aligned}
$$

A potência e a densidade dos precursores passam a ser governadas pelas equações A.1 e A.2. Portanto, aplicando as perturbações acima nas equações e usando o método de perturbação de primeira ordem resultam na seguintes equações das flutuações da potência e da densidade dos precursores:

$$
l_{\text {eff }} \frac{d\left(\delta P_{N}(t)\right)}{d t}=\left(\rho_{\text {gen }, o}-\alpha_{p d} \beta_{\text {eff }}-\zeta\right) \delta P_{N}(t)+\alpha_{p d} \sum_{i=1}^{I} \lambda_{i} \delta \xi_{i}(t)+\delta \rho_{g e n} P_{N, o}+\delta \rho_{\text {source }}
$$

$\mathrm{e}$

$\frac{d \delta \xi_{i}(t)}{d t}=\beta_{e f f, i} \cdot \delta P_{N}(t)-\lambda_{i} \cdot \delta \xi_{i}(t)$

Ao aplicar a transformada de Fourier nas duas equações acima, 
$-i 2 \pi f \cdot l_{\text {eff }} \cdot F\left\{\delta P_{N}(t)\right\}=\left(\rho_{\text {gen,o }}-\alpha_{p d} \beta_{\text {eff }}-\zeta\right) F\left\{\delta P_{N}(t)\right\}+\alpha_{p d} \sum_{i=1}^{I} \lambda_{i} F\left\{\delta \xi_{i}(t)\right\}+F\left\{\delta \rho_{g e n} P_{N, o}+\delta \rho_{\text {source }}\right\}$

$-i 2 \pi f \cdot F\left\{\delta \xi_{i}(t)\right\}=\beta_{e f f, i} \cdot F\left\{\delta P_{N}(t)\right\}-\lambda_{i} \cdot F\left\{\delta \xi_{i}(t)\right\}$.

Portanto,

$$
\begin{aligned}
-i 2 \pi f \cdot l_{e f f} \cdot F\left\{\delta P_{N}(t)\right\}= & \left(\rho_{\text {gen,o }}-\alpha_{p d} \beta_{e f f}-\zeta\right) F\left\{\delta P_{N}(t)\right\}+\alpha_{p d} \sum_{i=1}^{I} \lambda_{i} \frac{\beta_{e f f, i} \cdot F\left\{\delta P_{N}(t)\right\}}{\left(\lambda_{i}-i 2 \pi f\right)} \\
& +F\left\{\delta \rho_{g e n} P_{N, o}+\delta \rho_{\text {source }}\right\} .
\end{aligned}
$$

Finalmente, chega-se à expressão da função transferência (Diniz, 2005):

$$
G(f) \equiv \frac{F\left\{\delta P_{N}(t)\right\}}{F\left\{\delta \rho_{\text {gen }} \cdot P_{N, o}+\delta \rho_{\text {source }}\right\}}=\frac{1}{\alpha_{p d} \sum_{j=1}^{6} \frac{\lambda_{j} \beta_{e f f, j}}{\left(i 2 \pi f-\lambda_{j}\right)}-i 2 \pi f l_{e f f}-\rho_{g e n}+\zeta+\alpha_{p d} \beta} .
$$




\section{APÊNDICE B.}

\section{Dedução da expressão da potência relativa, $\boldsymbol{P}_{N}$.}

A APSD pode ser obtida seguindo procedimentos similar aos extremamente discutidos nas Referências (Cohn, 1960) e (Suzuki, 1966). O resultado final pode ser escrito como:

$$
\begin{aligned}
\Phi_{k k}(f) & =\left|\frac{1}{\alpha_{p d} \sum_{j=1}^{6} \frac{\lambda_{j} \beta_{e f f, j}}{\left(i 2 \pi f-\lambda_{j}\right)}-i 2 \pi f l_{e f f}-\rho_{g e n}+\zeta+\alpha_{p d} \beta}\right| \cdot \frac{2 N D v}{l_{e f f}} \cdot\left(\frac{\varepsilon_{k} q_{k}}{l_{e f f}}\right)^{2} \cdot\left(H_{e}(f) H_{f}(f)\right)^{2} \\
& +\frac{2 q_{k}^{2} \varepsilon_{k} N}{v l_{e f f}} \cdot\left(H_{e}(f) H_{f}(f)\right)^{2}
\end{aligned}
$$

onde :

$\Phi_{k k}(f)$ representa a APSD,

$D$ representa o fator de Diven,

$\checkmark$ representa o número médio de nêutrons emitidos por fissão,

$N$ representa o número total de nêutrons,

$\varepsilon_{k}$ representa a eficiência de detectores $k$, e

$q_{k}$ representa a carga média produzida por nêutron detectado,

$H_{e}(f)$ é a função transferência de "scaler" em Volt/contagem para detectores de modo pulso, e

$H_{f}(f)$ é a função transferência de demais módulos eletrônicos.

Considerando somente o termo dentro das barras de valor absoluto: 


$$
\left|\frac{1}{\alpha_{p d} \sum_{j=1}^{6} \frac{\lambda_{j} \beta_{e f f, j}}{\left(i 2 \pi f-\lambda_{j}\right)}-i 2 \pi f l_{e f f}-\rho_{g e n}+\zeta+\alpha_{p d} \beta}\right|^{2}
$$

e assumindo que $f>>\lambda$, o primeiro termo no denominador pode ser desconsiderada. A saber:

$$
\sum_{j=1}^{6} \frac{\lambda_{j} \beta_{e f f, j}}{\left(i 2 \pi f-\lambda_{j}\right)} \approx 0 .
$$

Portanto, o termo B.2 pode ser aproximado da seguinte maneira:

$$
\begin{aligned}
& \left|\frac{1}{\alpha_{p d} \sum_{j=1}^{6} \frac{\lambda_{j} \beta_{e f f, j}}{\left(i 2 \pi f-\lambda_{j}\right)}-i 2 \pi f l_{e f f}-\rho_{g e n}+\zeta+\alpha_{p d} \beta}\right| \approx l_{e f f}^{2} \cdot\left|\frac{-\left(\rho_{g e n}-\zeta-\alpha_{p d} \beta\right)}{l_{e f f}}-i 2 \pi f\right|^{2} \\
& \approx l_{e f f}^{2} \cdot\left|\frac{\left(\rho_{g e n}-\zeta-\alpha_{p d} \beta\right)}{l_{e f f}}+i 2 \pi f\right|^{2} \approx l_{e f f}^{2} \cdot\left[\frac{\left|\rho_{g e n}-\zeta-\alpha_{p d} \beta\right|^{2}}{l_{e f f}}+(2 \pi f)^{2}\right] .
\end{aligned}
$$

Assim, B.1 torna-se:

$$
\begin{aligned}
& \Phi(f)=\frac{1}{l_{e f f}^{2} \cdot\left[\frac{\left|\rho_{g e n}-\zeta-\alpha_{p d} \beta\right|^{2}}{l_{e f f}}+(2 \pi f)^{2}\right]} \cdot \frac{2 N D v}{l_{e f f}} \cdot\left(\frac{\varepsilon_{k} q_{k}}{v l_{e f f}}\right)^{2} \cdot\left(H_{e}(f) H_{f}(f)\right)^{2} \\
& +\frac{2 q_{k}^{2} \varepsilon_{k} N}{v l_{e f f}} \cdot\left(H_{e}(f) H_{f}(f)\right)^{2} .
\end{aligned}
$$


Agora, assumindo as seguintes definições para $A, B$ e $C$ :

$$
\begin{aligned}
& A \equiv \frac{2 N D v}{l_{e f f}} \cdot\left(\frac{\varepsilon_{k} q_{k}}{v_{e f f}}\right)^{2} \cdot\left(H_{e}(f) H_{f}(f)\right)^{2} ; \\
& C \equiv \frac{2 q_{k}^{2} \varepsilon_{k} N}{v l_{e f f}} \cdot\left(H_{e}(f) H_{f}(f)\right)^{2} ; \\
& B \equiv \frac{\left|\rho_{g e n}-\zeta-\alpha_{p d} \beta\right|}{l_{e f f}}
\end{aligned}
$$

e a relação:

$$
\omega=2 \pi f
$$

Então a APSD pode ser expressa numa forma mais simples como:

$$
\Phi(\omega)=\frac{A}{B^{2}+\omega^{2}}+C .
$$

E usando a seguinte relação (Diniz e Dos Santos, et al., 2006):

$$
\frac{R}{N}=\frac{\varepsilon_{k}}{v \cdot l_{\text {eff }}},
$$

onde $R$ é contagem do detector.

Agora, $A$ e $C$ podem ser escritos como sendo: 


$$
A=\frac{2 v D}{l_{e f f}^{3} N} \cdot R^{2} \cdot q_{k}^{2} \cdot\left(H_{e}(\omega) H_{f}(\omega)\right)^{2}
$$

e

$$
C=2 R \cdot q_{k}^{2} \cdot\left(H_{e}(\omega) H_{f}(\omega)\right)^{2}
$$

B. 11

Além disso, aplicando a relação (Dos Santos et al., 2004b);

$$
\frac{\gamma}{P \cdot l_{e f f}^{2}}=\frac{v}{N \cdot l_{e f f}}
$$

onde $P$ é a potência do reator e $\gamma$ energia liberada por fissão em Joule.

Então $A$ pode ser escrito da seguinte forma:

$$
A=\frac{2 \gamma D}{l_{e f f}^{4} P} \cdot R^{2} \cdot q_{k}^{2} \cdot\left(H_{e}(\omega) H_{f}(\omega)\right)^{2} .
$$

Além disso, de B.8, $A$ pode ser escrita ainda como:

$$
A=(\Phi(\omega)-C)\left(B^{2}+\omega^{2}\right)
$$

Portanto, a seguinte equação pode ser estabelecida:

$$
(\Phi(\omega)-C)\left(B^{2}+\omega^{2}\right)=\frac{2 \gamma D}{l_{e f f}^{4} P} \cdot R^{2} \cdot q_{k}^{2} \cdot\left(H_{e}(\omega) H_{f}(\omega)\right)^{2}
$$

Isolado $P$ de B.12: 


$$
P=\frac{\frac{2 \gamma D}{l_{\text {eff }}^{4}} \cdot R^{2} \cdot q_{k}^{2} \cdot\left(H_{e}(\omega) H_{f}(\omega)\right)^{2}}{(\Phi(\omega)-C)\left(B^{2}+\omega^{2}\right)} .
$$

Por conseguinte, a potência relativa da configuração $b$ em relação à configuração anterior, $a$, pode ser escrita como:

$$
P_{N}=\frac{P_{b}}{P_{a}}=\frac{\frac{\frac{2 \gamma D}{l_{e f f}^{4}} \cdot R_{b}^{2} \cdot q_{k}^{2} \cdot\left(H_{e}(\omega) H_{f}(\omega)\right)^{2}}{\left(\Phi_{b}(\omega)-C_{b}\right)\left(B_{b}^{2}+\omega^{2}\right)}}{\frac{\frac{2 \gamma D}{l_{e f f}^{4}} \cdot R_{a}^{2} \cdot q_{k}^{2} \cdot\left(H_{e}(\omega) H_{f}(\omega)\right)^{2}}{\left(\Phi_{a}(\omega)-C_{a}\right)\left(B_{a}^{2}+\omega^{2}\right)}}=\frac{R_{b}^{2}\left(B_{a}^{2}+\omega^{2}\right)\left(\Phi_{a}(\omega)-C_{a}\right)}{R_{a}^{2}\left(B_{b}^{2}+\omega^{2}\right)\left(\Phi_{b}(\omega)-C_{b}\right)} .
$$

E finalmente, dentro da região onde $B>>\omega, P_{N}$ torna-se:

$$
P_{N}=\frac{R_{b}^{2} B_{a}^{2}\left(\Phi_{a}^{p}(\omega)-C_{a}\right)}{R_{a}^{2} B_{b}^{2}\left(\Phi_{b}^{p}(\omega)-C_{b}\right)} .
$$

onde $\Phi^{p}$ representa a média da APSD no plateau superior. 


\section{APÊNDICE C.}

\section{Procedimento Experimental}

A seguir, são expostos, de maneira resumida, os procedimentos experimentais dos quatro últimos experimentos realizados no Reator IPEN/MB-01.

1- Posicionar o detector $\mathrm{BF}_{3}$ na face oeste do núcleo do reator.

2- Posicionar a fonte externa de Am-Be, de intensidade de $100 \mathrm{mCi}$ na posição lateral leste do núcleo do reator

3- Inserir a fonte de partida no núcleo, preencher o tanque moderador com água e retirar completamente as barras de segurança BS1 e BS2.

4- Verificar a temperatura do moderador com termopares posicionados no interior do núcleo. Se necessário deve-se aquecer ou resfriar a água de modo que a temperatura permaneça próxima $\operatorname{dos} 21^{\circ} \mathrm{C} \pm 0,5^{\circ} \mathrm{C}$.

5- Remover as duas barras de controle $\mathrm{BC} 1$ e $\mathrm{BC} 2$ até atingir a configuração inicial que apresenta um pequeno excesso de reatividade $(\sim 10 \mathrm{pcm})$.

6- Esperar até o fluxo estabilizar, e uma vez estabilizado o fluxo, iniciar a aquisição de dados referentes à medida de APSD utilizando o software LabVIEW 5.1 durante $1 / 2$ a 1 hora.

7- Após o término da aquisição, inserir as duas barras de controle $\mathrm{BC} 1$ e $\mathrm{BC} 2$ em passos de $2,5 \%$ ou $5 \%$, dependendo dos resultados obtidos. 
8- Repetir os itens 5 e 6 até a taxa de contagem chegar perto do limiar de detecção do detector $\mathrm{BF}_{3}$.

9- Trocar o detector $\mathrm{BF}_{3}$ por um de ${ }^{3} \mathrm{He}$ de sensibilidade média e repetir os itens 5 e 6, começando a partir da antepenúltima posição das duas barras de controle, até a taxa de contagem chegar perto do limite do detector ${ }^{3} \mathrm{He}$ de sensibilidade média.

10-Trocar o detector ${ }^{3} \mathrm{He}$ de sensibilidade média por outro de ${ }^{3} \mathrm{He}$ de sensibilidade alta e repetir os itens 5 e 6 , começando a partir da antepenúltima posição das duas barras de controle, até a taxa de contagem chegar perto do limite do detector.

11- Repetir os itens de 1 a 9 com uma fonte externa de Am-Be, de intensidade de 1Ci na mesma posição.

12- Repetir o item $11 \mathrm{com}$ duas fontes externas de Am-Be, de intensidade 1Ci na mesma posição do item 11. 


\section{APÊEDICE D.1..}

Potência relativa, reatividade parcial e total, e o índice de subcriticalidade obtidos por APSD(1); com o detector $\mathrm{BF}_{3}$ e o detector ${ }^{3} \mathrm{He}$ de sensibilidade alta.

\begin{tabular}{|c|c|c|c|c|c|c|c|c|}
\hline $\begin{array}{c}\text { Posição } \\
\text { dos } \\
\text { BCs } \\
(\%)\end{array}$ & $\boldsymbol{P}_{N}$ & $\sigma_{P N}$ & $\rho_{g e n}^{(1)}$ & $\sigma_{\rho g e n}$ & $\Sigma \boldsymbol{\rho}_{\text {gen }}(\mathrm{pcm})$ & $\begin{array}{c}\boldsymbol{\sigma}_{\boldsymbol{\Sigma} \boldsymbol{\rho}} \\
(\mathrm{pcm})\end{array}$ & $\zeta(\mathrm{pcm})$ & $\boldsymbol{\sigma}_{\zeta}(\mathrm{pcm})$ \\
\hline 100,0 & & & 10,00 & 2,97 & & & & \\
\hline 93,0 & & & $-118,86$ & 18,20 & $-108,86$ & 18,44 & & \\
\hline 90,5 & 0,618 & 0,016 & $-78,45$ & 9,19 & $-187,31$ & 20,60 & 127,00 & 11,77 \\
\hline 88,0 & 0,692 & 0,018 & $-92,97$ & 10,10 & $-280,28$ & 22,95 & 208,95 & 14,48 \\
\hline 85,5 & 0,723 & 0,020 & $-108,67$ & 11,99 & $-388,95$ & 25,89 & 283,18 & 16,53 \\
\hline 83,0 & 0,823 & 0,025 & $-86,77$ & 14,58 & $-475,72$ & 29,71 & 402,20 & 20,75 \\
\hline 80,5 & 0,778 & 0,025 & $-137,04$ & 19,29 & $-612,77$ & 35,42 & 481,07 & 22,46 \\
\hline 78,0 & 0,879 & 0,031 & $-87,12$ & 24,20 & $-699,89$ & 42,90 & 631,70 & 28,18 \\
\hline 75,5 & 0,838 & 0,033 & $-143,07$ & 32,17 & $-842,96$ & 53,62 & 742,68 & 31,81 \\
\hline 73,0 & 0,825 & 0,035 & $-169,34$ & 38,39 & $-1012,30$ & 65,95 & 798,63 & 34,97 \\
\hline 70,5 & 0,846 & 0,012 & $-189,59$ & 16,47 & $-1201,89$ & 67,97 & 1044,11 & 32,41 \\
\hline 68,0 & 0,884 & 0,013 & $-161,97$ & 19,09 & $-1363,86$ & 70,60 & 1238,24 & 36,99 \\
\hline 65,5 & 0,868 & 0,013 & $-211,65$ & 22,84 & $-1575,51$ & 74,21 & 1390,55 & 40,13 \\
\hline 63,0 & 0,801 & 0,014 & $-354,17$ & 27,11 & $-1929,68$ & 79,01 & 1423,83 & 40,39 \\
\hline 60,5 & 0,849 & 0,015 & $-298,69$ & 32,10 & $-2228,37$ & 85,28 & 1681,09 & 47,28 \\
\hline 58,0 & 0,833 & 0,015 & $-368,72$ & 37,05 & $-2597,09$ & 92,98 & 1845,27 & 51,24 \\
\hline 55,5 & 0,855 & 0,016 & $-356,29$ & 42,84 & $-2953,38$ & 102,37 & 2092,66 & 57,66 \\
\hline 53,0 & 0,881 & 0,018 & $-315,88$ & 49,25 & $-3269,26$ & 113,60 & 2336,39 & 64,33 \\
\hline 50,5 & 0,869 & 0,018 & $-385,68$ & 57,15 & $-3654,94$ & 127,17 & 2547,66 & 70,39 \\
\hline 48,0 & 0,908 & 0,020 & $-287,07$ & 66,31 & $-3942,01$ & 143,42 & 2843,82 & 79,50 \\
\hline 45,5 & 0,910 & 0,022 & $-299,40$ & 75,24 & $-4241,42$ & 161,96 & 3041,71 & 86,52 \\
\hline 43,0 & 0,902 & 0,023 & $-348,89$ & 85,02 & $-4590,31$ & 182,91 & 3209,85 & 92,98 \\
\hline 40,5 & 0,933 & 0,025 & $-249,92$ & 96,50 & $-4840,22$ & 206,81 & 3475,49 & 103,44 \\
\hline
\end{tabular}

(1) A reatividade generalizada parcial na posição de $93 \%$ foi calculada pelo modelo da cinética pontual clássica; a da $100 \%$ foi medido pelo reatímetro do modelo de cinética inversa. 


\section{APÊEDICE D.2.}

Potência relativa, reatividade parcial e total, e o índice de subcriticalidade obtidos por $\operatorname{APSD}(2)$; com o detector $\mathrm{BF}_{3}$ e o detector ${ }^{3} \mathrm{He}$ de sensibilidade média.

\begin{tabular}{|c|c|c|c|c|c|c|c|c|}
\hline $\begin{array}{c}\text { Posição } \\
\text { dos } \\
\text { BCs } \\
(\%)\end{array}$ & $\boldsymbol{P}_{N}$ & $\sigma_{P N}$ & $\rho_{g e n}^{(1)}$ & $\sigma_{\rho g e n}$ & $\boldsymbol{\Sigma} \boldsymbol{\rho}_{\text {gen }}(\mathrm{pcm})$ & $\begin{array}{c}\boldsymbol{\sigma}_{\boldsymbol{\Sigma} \boldsymbol{\rho}} \\
(\mathrm{pcm})\end{array}$ & $\zeta(\mathrm{pcm})$ & $\boldsymbol{\sigma}_{\zeta}(\mathrm{pcm})$ \\
\hline 100,0 & & & 10,00 & 2,97 & & & & \\
\hline 93,0 & & & $-120,20$ & 18,19 & $-110,20$ & 18,43 & & \\
\hline 90,5 & 0,592 & 0,015 & $-93,65$ & 9,92 & $-203,85$ & 20,93 & 136,06 & 11,52 \\
\hline 88,0 & 0,687 & 0,018 & $-90,03$ & 9,83 & $-293,88$ & 23,12 & 197,40 & 14,15 \\
\hline 85,5 & 0,796 & 0,022 & $-78,51$ & 11,09 & $-372,39$ & 25,64 & 305,43 & 18,05 \\
\hline 83,0 & 0,748 & 0,022 & $-126,63$ & 14,81 & $-499,02$ & 29,61 & 374,91 & 19,02 \\
\hline 80,5 & 0,768 & 0,024 & $-149,75$ & 19,46 & $-648,77$ & 35,43 & 495,48 & 22,48 \\
\hline 78,0 & 0,865 & 0,029 & $-100,08$ & 24,02 & $-748,85$ & 42,81 & 640,49 & 28,04 \\
\hline 75,5 & 0,840 & 0,031 & $-140,92$ & 30,53 & $-889,78$ & 52,58 & 738,60 & 31,29 \\
\hline 73,0 & 0,827 & 0,034 & $-167,99$ & 36,86 & $-1057,76$ & 64,21 & 803,56 & 34,35 \\
\hline 70,5 & 0,853 & 0,019 & $-181,84$ & 25,58 & $-1239,61$ & 69,12 & 1054,12 & 35,04 \\
\hline 68,0 & 0,882 & 0,021 & $-169,36$ & 31,94 & $-1408,96$ & 76,14 & 1270,20 & 41,17 \\
\hline 65,5 & 0,884 & 0,022 & $-191,78$ & 39,23 & $-1600,75$ & 85,66 & 1466,57 & 46,99 \\
\hline 63,0 & 0,847 & 0,022 & $-293,47$ & 46,66 & $-1894,22$ & 97,54 & 1629,20 & 51,92 \\
\hline 60,5 & 0,856 & 0,024 & $-310,17$ & 54,85 & $-2204,39$ & 111,90 & 1848,59 & 58,69 \\
\hline 58,0 & 0,878 & 0,026 & $-290,84$ & 64,97 & $-2495,23$ & 129,39 & 2088,96 & 67,09 \\
\hline 55,5 & 0,912 & 0,029 & $-222,47$ & 75,38 & $-2717,70$ & 149,75 & 2299,44 & 75,82 \\
\hline 53,0 & 0,900 & 0,030 & $-268,52$ & 84,91 & $-2986,22$ & 172,15 & 2411,43 & 82,05 \\
\hline 50,5 & 0,922 & 0,033 & $-227,04$ & 99,23 & $-3213,26$ & 198,70 & 2669,56 & 93,53 \\
\hline 48,0 & 0,833 & 0,033 & $-548,23$ & 114,54 & $-3761,48$ & 229,35 & 2725,77 & 98,96 \\
\hline 45,5 & 0,944 & 0,040 & $-195,60$ & 141,18 & $-3957,09$ & 269,32 & 3300,88 & 126,32 \\
\hline 43,0 & 0,860 & 0,038 & $-516,44$ & 148,10 & $-4473,53$ & 307,35 & 3178,65 & 125,88 \\
\hline 40,5 & 1,098 & 0,053 & 358,33 & 189,95 & $-4115,20$ & 361,31 & 4032,96 & 166,99 \\
\hline
\end{tabular}

(1) A reatividade generalizada parcial na posição de $93 \%$ foi calculada pelo modelo da cinética pontual clássica; a da $100 \%$ foi medido pelo reatímetro do modelo de cinética inversa. 


\section{APÊNDICE E}

Interpolações dos valores do $\Sigma \rho_{g e n}$ e $\zeta$ do GPT-TORT nas posições dos experimentos, por meio de ajuste de funções polinomiais de sexta ordem.

\section{E.1. Reatividade generalizada total, $\Sigma \rho_{g e n}$;}

Função de Ajuste: $\quad R(x)=A_{0}+A_{1} x^{1}+A_{2} x^{2}+A_{3} x^{3}+A_{4} x^{4}+A_{5} x^{5}+A_{6} x^{6}$ (onde $x$ é posição dos BCs em \%)

\begin{tabular}{ccc}
\hline Coeficiente & Valor & Erro \\
\hline $\mathrm{A}_{0}$ & $4.02751 \mathrm{E}+01$ & $7.88469 \mathrm{E}+00$ \\
$\mathrm{~A}_{1}$ & $-1.06556 \mathrm{E}+00$ & $7.16570 \mathrm{E}-01$ \\
$\mathrm{~A}_{2}$ & $1.64580 \mathrm{E}-01$ & $2.81400 \mathrm{E}-02$ \\
$\mathrm{~A}_{3}$ & $-3.70000 \mathrm{E}-03$ & $5.40000 \mathrm{E}-04$ \\
$\mathrm{~A}_{4}$ & $3.00000 \mathrm{E}-05$ & $4.88460 \mathrm{E}-06$ \\
$\mathrm{~A}_{5}$ & $-1.00830 \mathrm{E}-07$ & $1.70400 \mathrm{E}-08$ \\
$\mathrm{~A}_{6}$ & $-8.49487 \mathrm{E}+03$ & $2.69848 \mathrm{E}+01$ \\
\hline
\end{tabular}

\section{E.2. Índice de subcriticalidade, $\zeta$;}

Função de Ajuste: $Z(x)=B_{0}+B_{1} x^{1}+B_{2} x^{2}+B_{3} x^{3}+B_{4} x^{4}+B_{5} x^{5}+B_{6} x^{6}$ (onde $x$ é posição dos BCs em \%)

\begin{tabular}{ccc}
\hline Coeficiente & \multicolumn{1}{c}{ Valor } & Erro \\
\hline $\mathrm{B}_{0}$ & $-1.17239 \mathrm{E}+01$ & $4.80716 \mathrm{E}+00$ \\
$\mathrm{~B}_{1}$ & $-1.48377 \mathrm{E}+00$ & $4.36880 \mathrm{E}-01$ \\
$\mathrm{~B}_{2}$ & $-8.39200 \mathrm{E}-02$ & $1.71500 \mathrm{E}-02$ \\
$\mathrm{~B}_{3}$ & $2.78000 \mathrm{E}-03$ & $3.30000 \mathrm{E}-04$ \\
$\mathrm{~B}_{4}$ & $-3.00000 \mathrm{E}-05$ & $2.97810 \mathrm{E}-06$ \\
$\mathrm{~B}_{5}$ & $1.00850 \mathrm{E}-07$ & $1.03890 \mathrm{E}-08$ \\
$\mathrm{~B}_{6}$ & $7.19143 \mathrm{E}+03$ & $1.64521 \mathrm{E}+01$ \\
\hline
\end{tabular}


E.3. Os gráficos das funções de ajustes: (a) de $\Sigma \rho_{g e n}$ e (b) de $\zeta$.

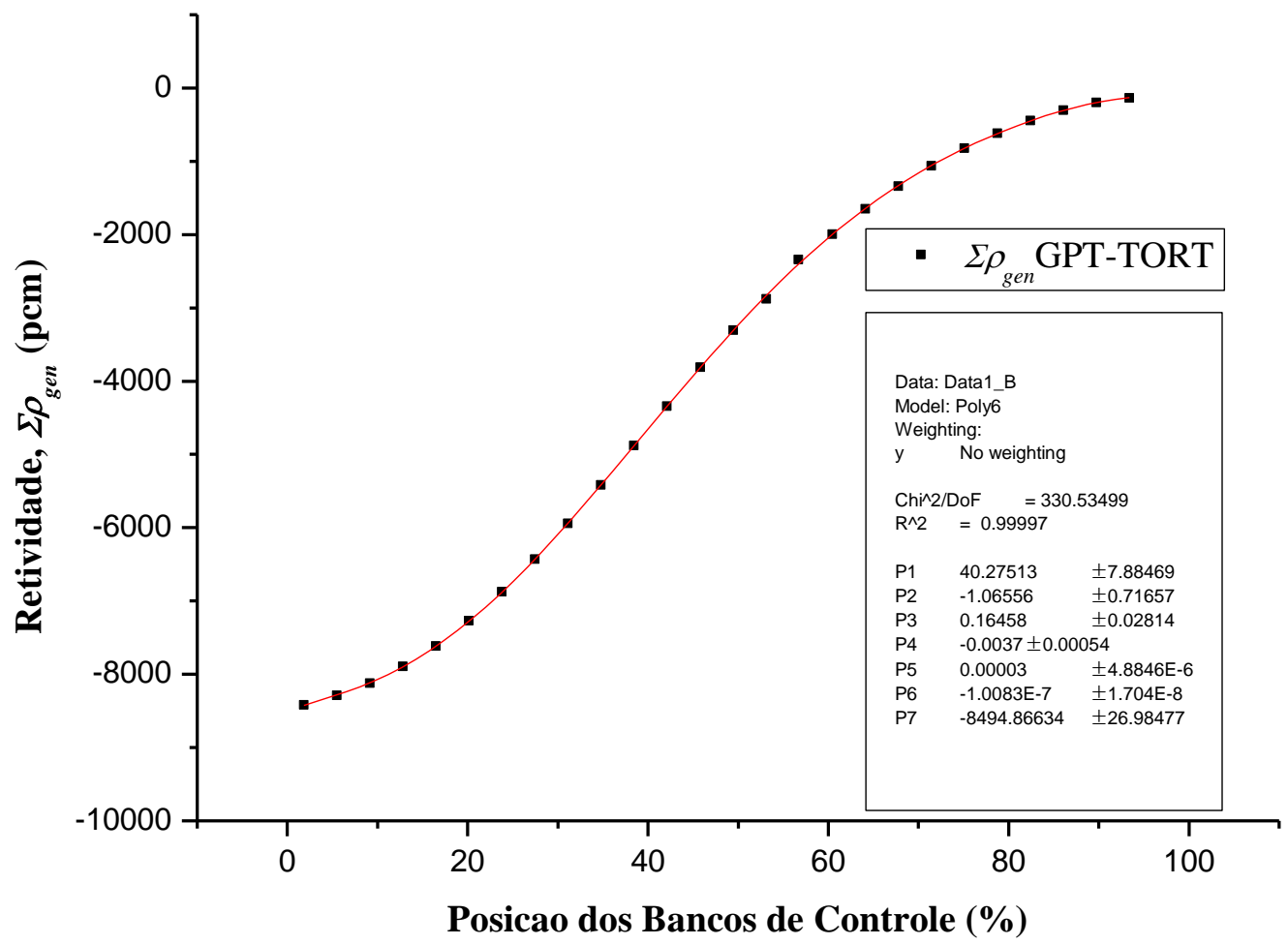

(a)

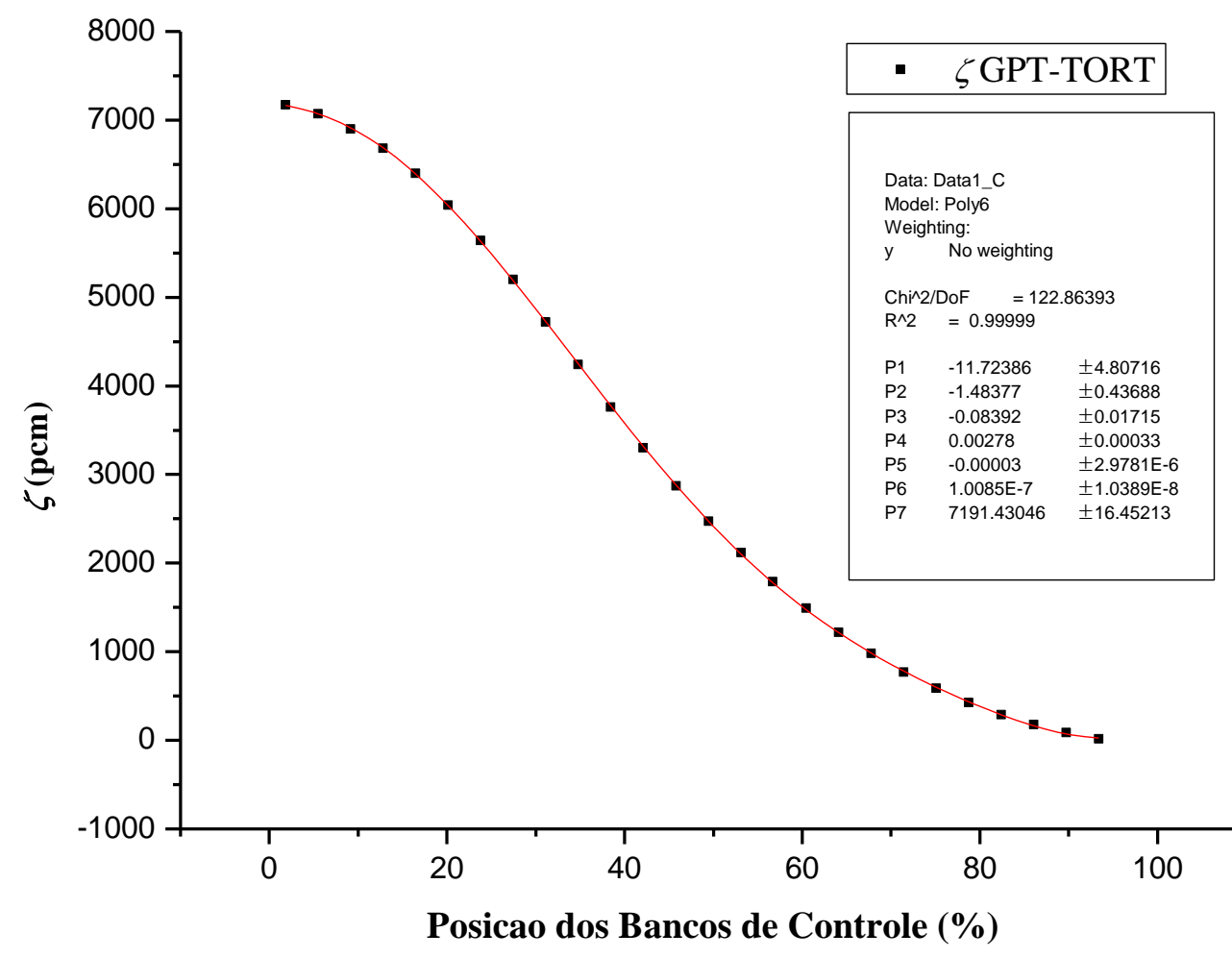

(b) 
E.4. Valores do $\Sigma \rho_{\text {gen }}$ e $\zeta$ do GPT-TORT interpolados.

\begin{tabular}{crr}
\hline Posição $(\boldsymbol{\%})$ & $\boldsymbol{\Sigma} \boldsymbol{\rho}_{\text {gen }}(\mathbf{p c m})$ & \multicolumn{1}{c}{$\boldsymbol{\zeta}(\mathbf{p c m})$} \\
\hline $\mathbf{9 3 , 0}$ & $-137,92369$ & 29,79399 \\
$\mathbf{9 0 , 5}$ & $-182,38033$ & 58,85425 \\
$\mathbf{8 8 , 0}$ & $-246,62575$ & 111,88192 \\
$\mathbf{8 5 , 5}$ & $-327,69043$ & 182,71388 \\
$\mathbf{8 3 , 0}$ & $-423,80679$ & 266,98782 \\
$\mathbf{8 0 , 5}$ & $-534,17066$ & 361,86205 \\
$\mathbf{7 8 , 0}$ & $-658,72037$ & 465,75302 \\
$\mathbf{7 5 , 5}$ & $-797,93361$ & 578,09053 \\
$\mathbf{7 3 , 0}$ & $-952,64201$ & 699,09078 \\
$\mathbf{7 0 , 5}$ & $-1123,86348$ & 829,54699 \\
$\mathbf{6 8 , 0}$ & $-1312,65218$ & 970,63788 \\
$\mathbf{6 5 , 5}$ & $-1519,96628$ & 1123,75381 \\
$\mathbf{6 3 , 0}$ & $-1746,55347$ & 1290,34068 \\
$\mathbf{6 0 , 5}$ & $-1992,85413$ & 1471,76151 \\
$\mathbf{5 8 , 0}$ & $-2258,92224$ & 1669,17578 \\
$\mathbf{5 5 , 5}$ & $-2544,36404$ & 1883,43654 \\
$\mathbf{5 3 , 0}$ & $-2848,29439$ & 2115,00512 \\
$\mathbf{5 0 , 5}$ & $-3169,31088$ & 2363,88372 \\
$\mathbf{4 8 , 0}$ & $-3505,48560$ & 2629,56562 \\
$\mathbf{4 5 , 5}$ & $-3854,37472$ & 2911,00313 \\
$\mathbf{4 3 , 0}$ & $-4213,04572$ & 3206,59336 \\
$\mathbf{4 0 , 5}$ & $-4578,12241$ & 3514,18154 \\
$\mathbf{3 8 , 0}$ & $-4945,84760$ & 3831,08226 \\
$\mathbf{3 5 , 5}$ & $-5312,16354$ & 4154,11831 \\
$\mathbf{3 3 , 0}$ & $-5672,81011$ & 4479,67728 \\
$\mathbf{3 0 , 5}$ & $-6023,44064$ & 4803,78592 \\
$\mathbf{2 8 , 0}$ & $-6359,75556$ & 5122,20214 \\
$\mathbf{2 5 , 5}$ & $-6677,65370$ & 5430,52487 \\
$\mathbf{2 3 , 0}$ & $-6973,40135$ & 5724,32152 \\
$\mathbf{2 0 , 5}$ & $-7243,81901$ & 5999,27324 \\
$\mathbf{1 8 , 0}$ & $-7486,48593$ & 6251,33786 \\
$\mathbf{1 5 , 5}$ & $-7699,96228$ & 6476,93063 \\
$\mathbf{1 3 , 0}$ & $-7884,02914$ & 6673,12260 \\
$\mathbf{1 0 , 5}$ & $-8039,94611$ & 6837,85677 \\
$\mathbf{8 , 0}$ & $-8170,72676$ & 6970,18197 \\
$\mathbf{5 , 5}$ & $-8281,43168$ & 7070,50447 \\
$\mathbf{0 , 0}$ & $-8494,86634$ & 7191,43046 \\
\hline & & \\
\hline
\end{tabular}




\section{APÊNDICE F.}

\section{Entrada de Dados Utilizados}

\section{F.1. Entrada de dados para MCNP5 com fonte intrínseca}

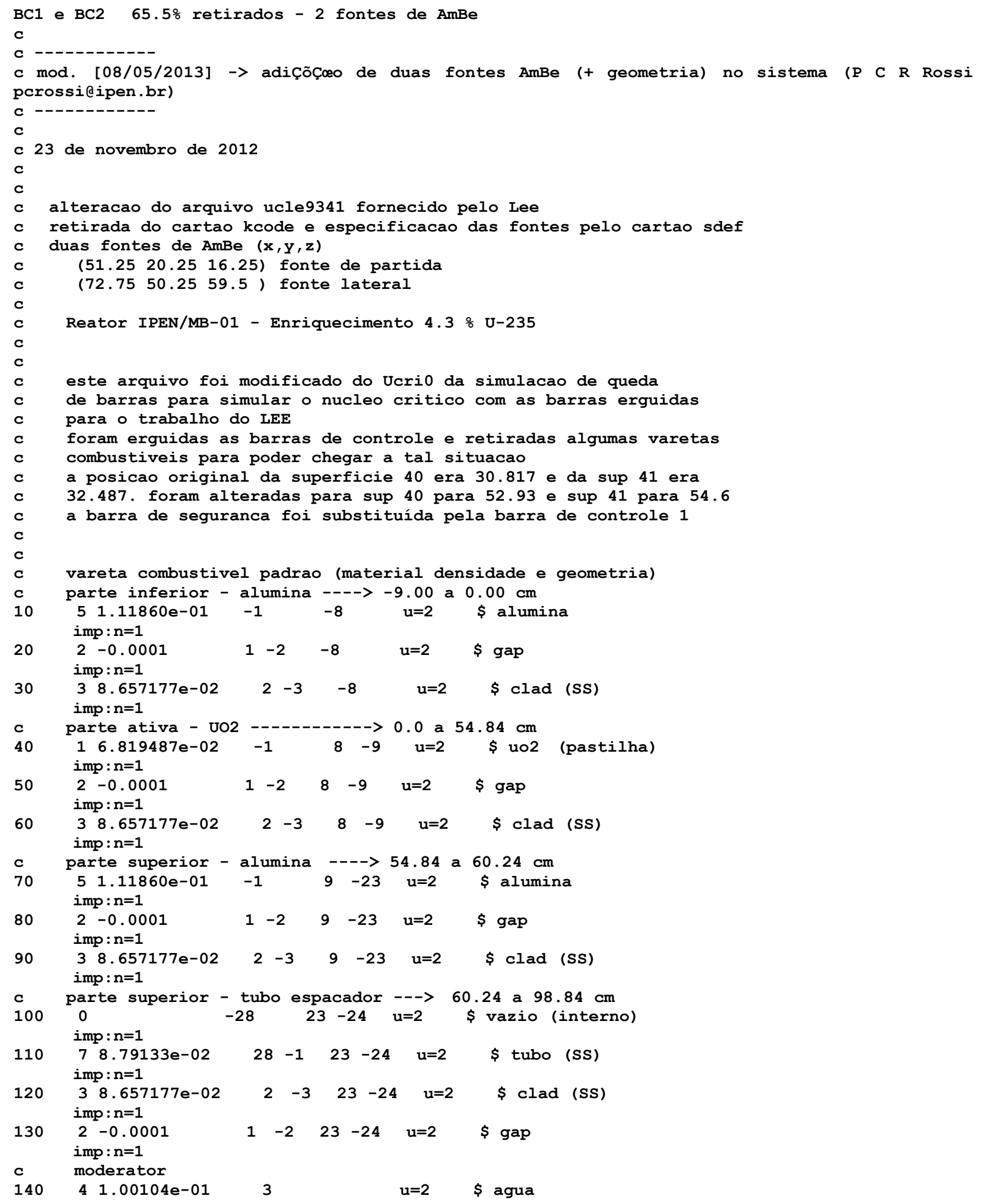




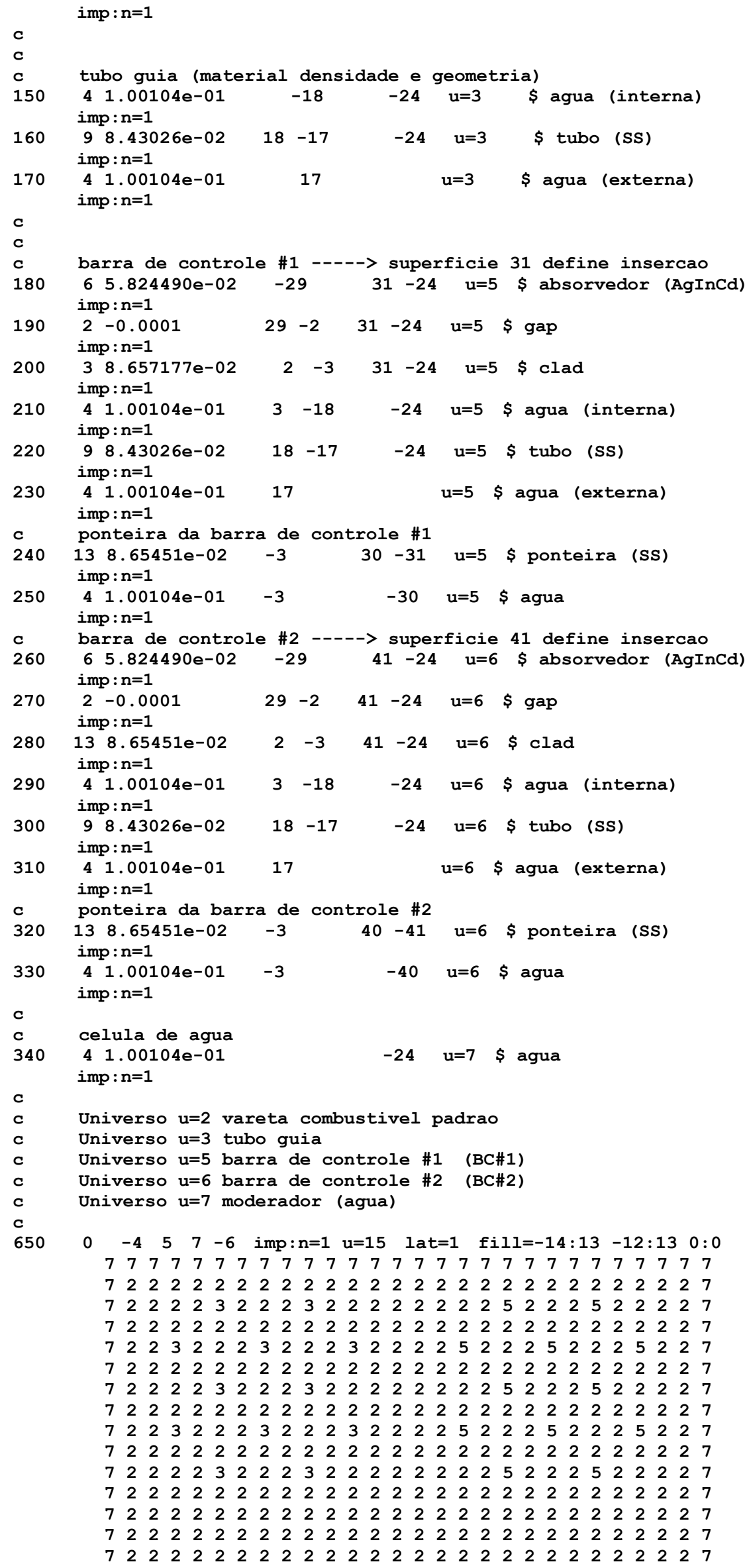




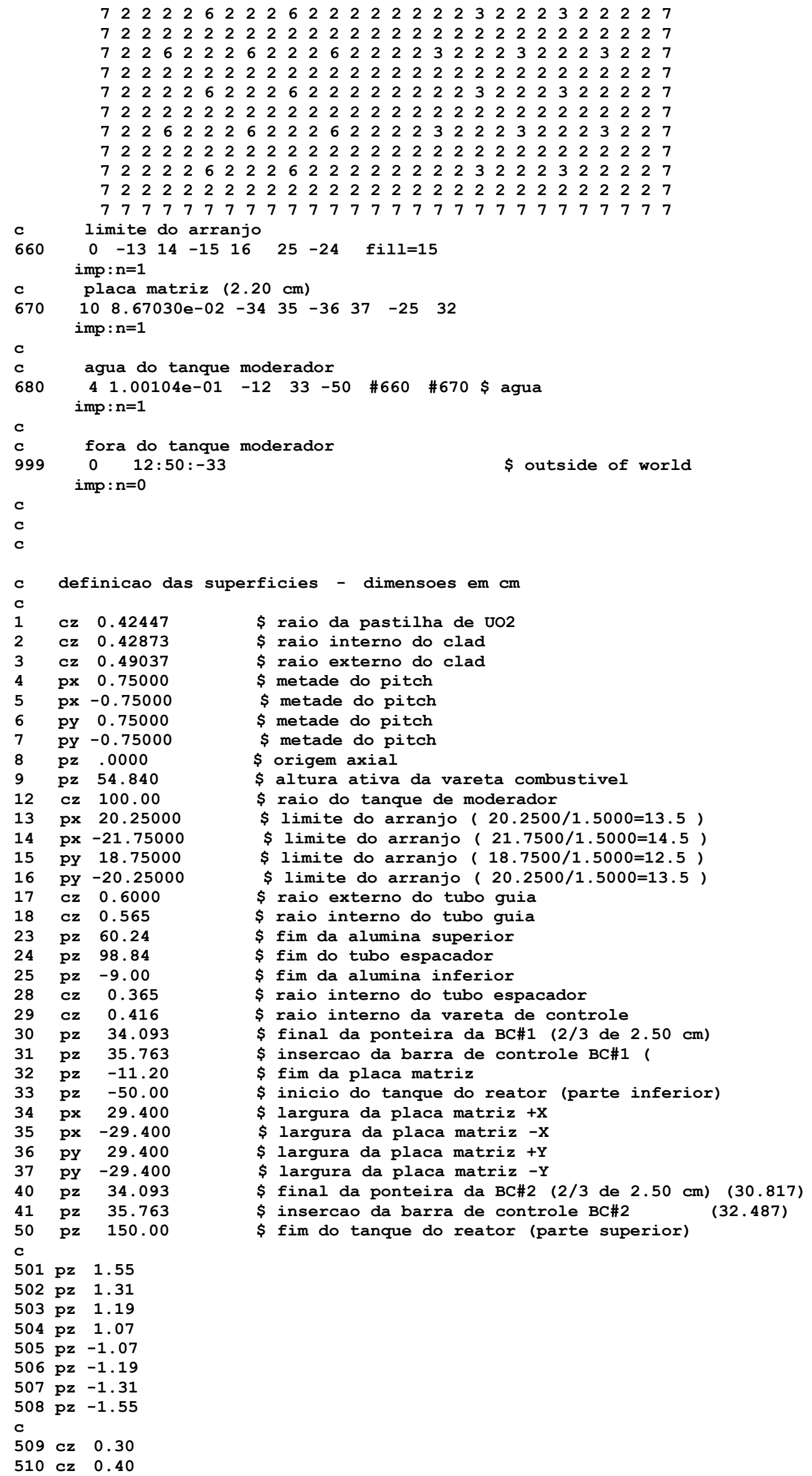




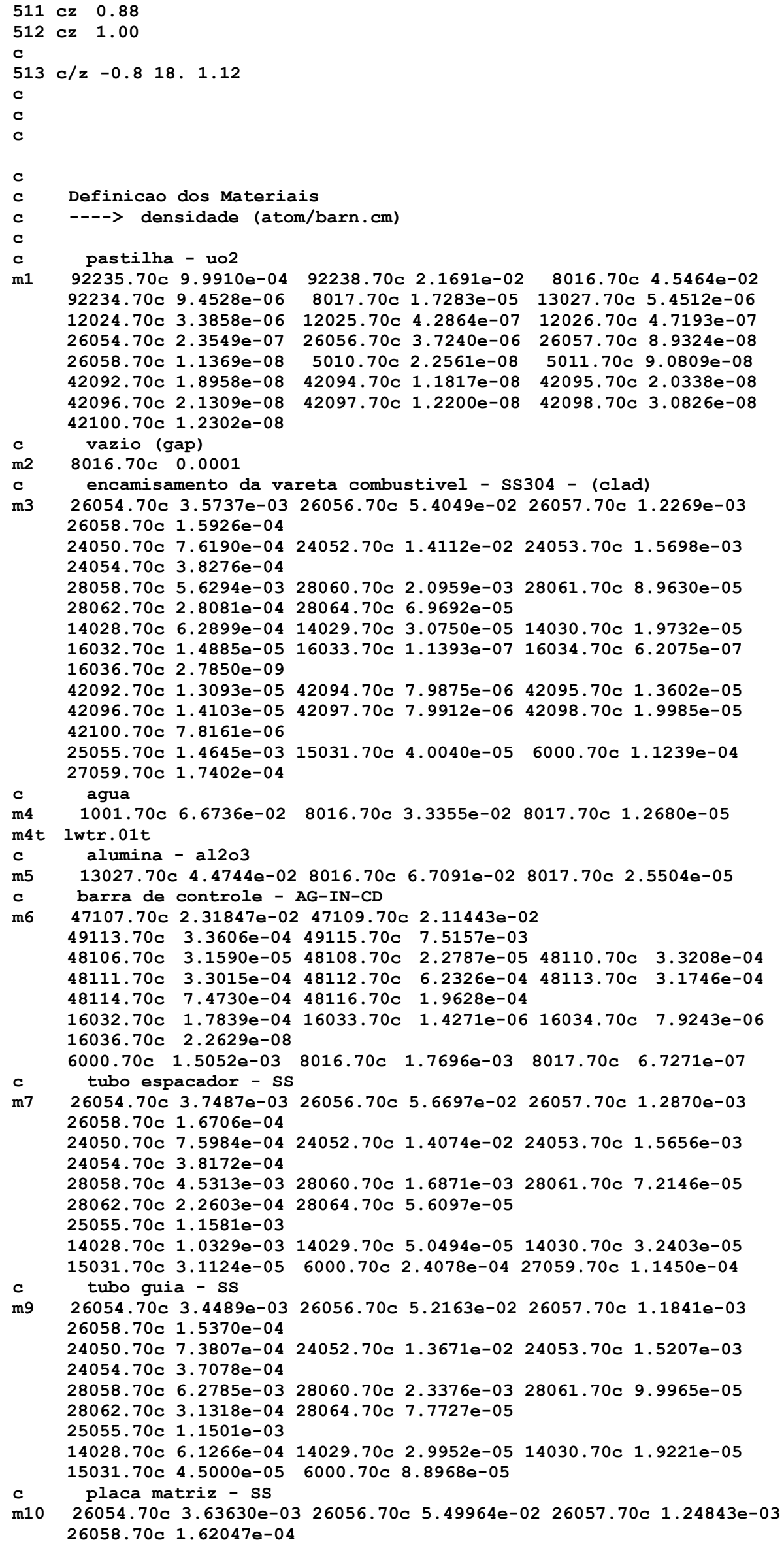


$24050.70 c 7.56501 e-0424052.70 c 1.40122 e-0224053.70 c \quad 1.55868 e-03$ $24054.70 c 3.80042 e-04$

28058.70 c $5.28566 e-0328060.70 c 1.96791 e-0328061.70 c 8.41568 e-05$

$28062.70 \mathrm{c} 2.63658 \mathrm{e}-0428064.70 \mathrm{c} 6.54358 \mathrm{e}-05$

14028.70 c $8.01962 e-0414029.70 c \quad 3.92060 e-0514030.70 c \quad 2.51595 e-05$

$16032.70 c 4.25282 e-0616033.70 c \quad 3.25504 e-08 \quad 16034.70 c \quad 1.77356 e-07$

16036.70 c $7.95715 e-10$

$42092.70 c \quad 4.62114 e-06 \quad 42094.70 c \quad 2.81913 e-06 \quad 42095.70 c \quad 4.80079 e-06$

$42096.70 c \quad 4.97759 e-06 \quad 42097.70 c 2.82043 e-06 \quad 42098.70 c \quad 7.05364 e-06$

$42100.70 \mathrm{c} 2.75861 \mathrm{e}-06$

$25055.70 c 1.25030 e-0315031.70 c 5.54400 e-05 \quad 6000.70 c 7.94260 e-05$

encamisamento das barras de controle e de seguranca - SS304

m13 26054.70c 3.50278e-03 26056.70c 5.29770e-02 26057.70c 1.20259e-03

26058.70 c $1.56097 e-04$

$24050.70 c 7.62733 e-0424052.70 c \quad 1.41277 e-02 \quad 24053.70 c \quad 1.57152 e-03$

$24054.70 \mathrm{c} 3.83172 \mathrm{e}-04$

$28058.70 c 6.54682 e-0328060.70 c 2.43746 e-0328061.70 c 1.04236 e-04$

28062.70 c $3.26566 e-0428064.70$ c $8.10488 e-05$

14028.70 c $7.0501 e-0414029.70$ c 3.5698e-05 $14030.70 c \quad 2.3697 e-05$

$16032.70 c 4.2412 e-0616033.70 c \quad 3.3476 e-08$ 16034.70c $1.8791 e-07$

$16036.70 \mathrm{c} 8.9270 \mathrm{e}-10$

42092.70 c 2.2150e-06 42094.70c 1.3806e-06 42095.70c 2.3762e-06

$42096.70 \mathrm{c} 2.4896 \mathrm{e}-06 \quad 42097.70 \mathrm{c} 1.4254 \mathrm{e}-06 \quad 42098.70 \mathrm{c} 3.6016 \mathrm{e}-06$

42100.70 c $1.4373 e-06$

$29063.70 c 9.43728 e-0529065.70 c 4.07687 e-05$

50112.70 c $3.11911-0850114.70 c \quad 2.0901 e-08$ 50115.70c $1.0933 e-08$

$50116.70 \mathrm{c} 4.6754 \mathrm{e}-0750117.70 \mathrm{c} 2.4695 \mathrm{e}-07 \quad 50118.70 \mathrm{c} 7.7881 \mathrm{e}-07$

50119.70 c 2.7622e-07 50120.70c $1.0479 e-0650122.70 c \quad 1.4888 e-07$

$50124.70 \mathrm{c} 1.8618 \mathrm{e}-07$

15031.70 c $4.15796 e-05 \quad 6000.70 c 8.33972 e-05 \quad 27059.70 c \quad 1.37594 e-04$

25055.70c $1.18080 \mathrm{e}-03$

C Berilio dens $=-1.848 \mathrm{~g} / \mathrm{CC}$ @ $20 \mathrm{C}$

m15 4009.70c 1

mt15 be.10t

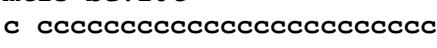

c ALUMINUM C

C $\operatorname{coc} c \operatorname{coc} c \operatorname{coc} c \operatorname{coc} \operatorname{coc}$

c

m20 $13027 \quad 6.0262 \mathrm{E}-02$

c

c fonte

c

mode $\mathrm{n}$

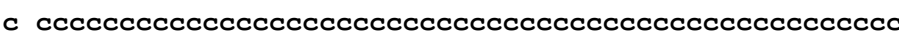

C SOURCE CARDS

sdef cel d4

pos $=0.00 .00 .0 \$$ center vol. source

axs $=\begin{array}{llllll}0 & 0 & 1 & \$ \text { axis vol source }\end{array}$

rad d1 \$ radial - distributions 1

ext d2 \$ axial - distributions 2

erg d3 \$ energy - distributions 3

sil h 0 0.42447 $\quad$ \$ radii vol. source

sp1 -211

si2 h 054.84 \$ extension of cylinder

sp3 -3 \$ Watt spectrum

si4 $1 \quad(40<650<660)$

sp4 1

C

C controle de execucao

nps 500000

prdmp j $50000-1$

print

c

c 


\section{F.2. Entrada de dados para MCNP5 com fonte externa de Am-Be}

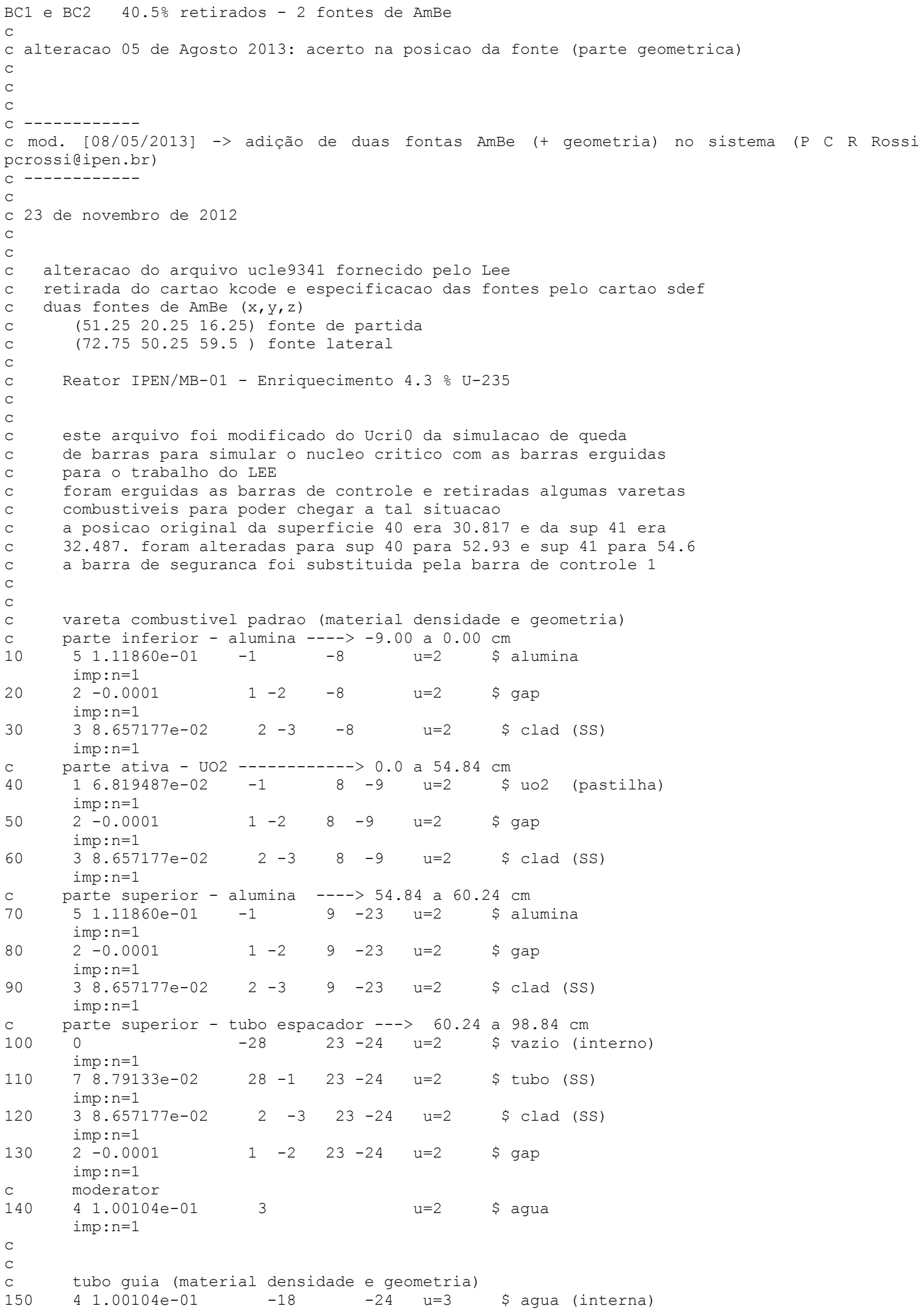




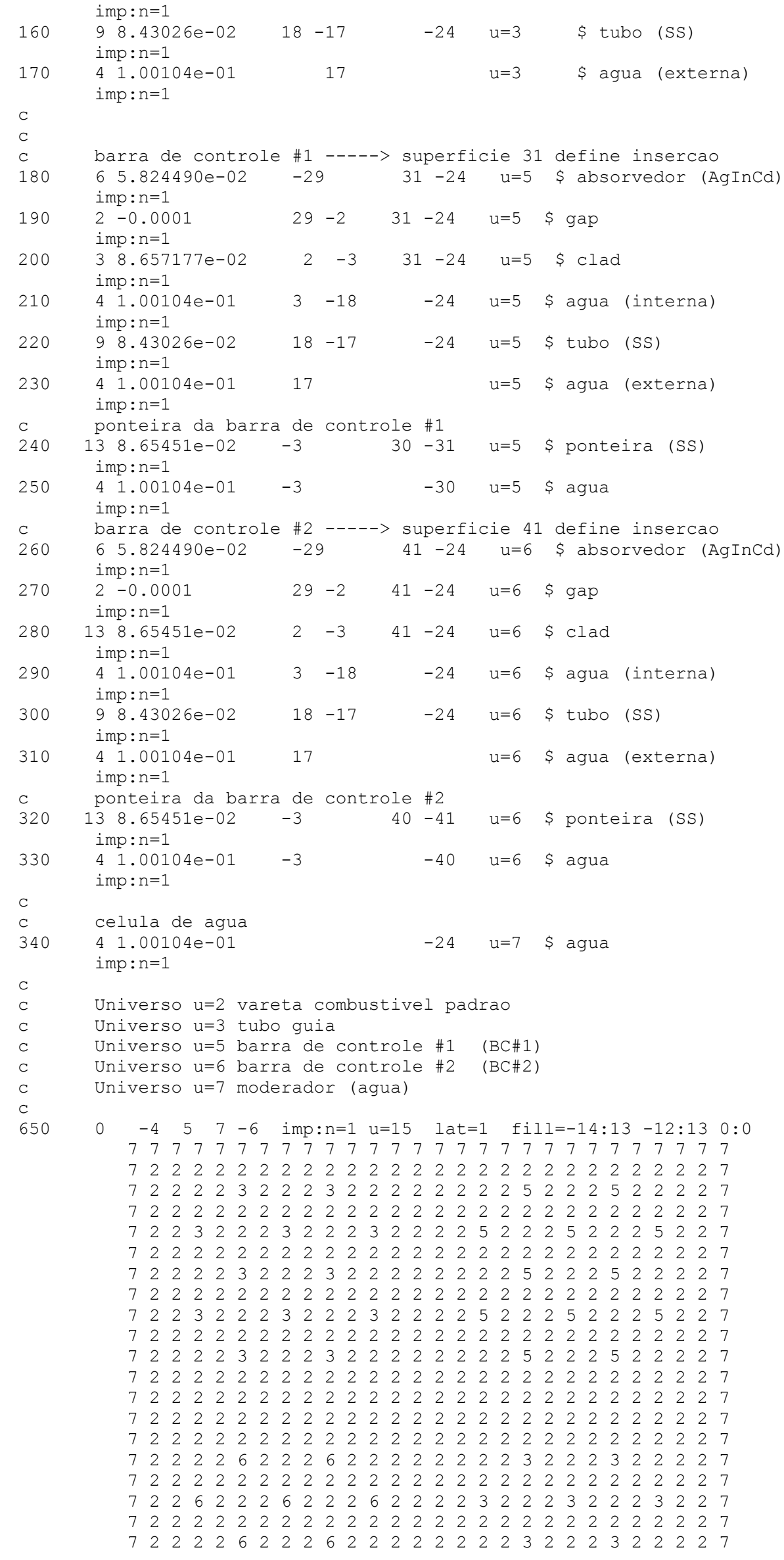




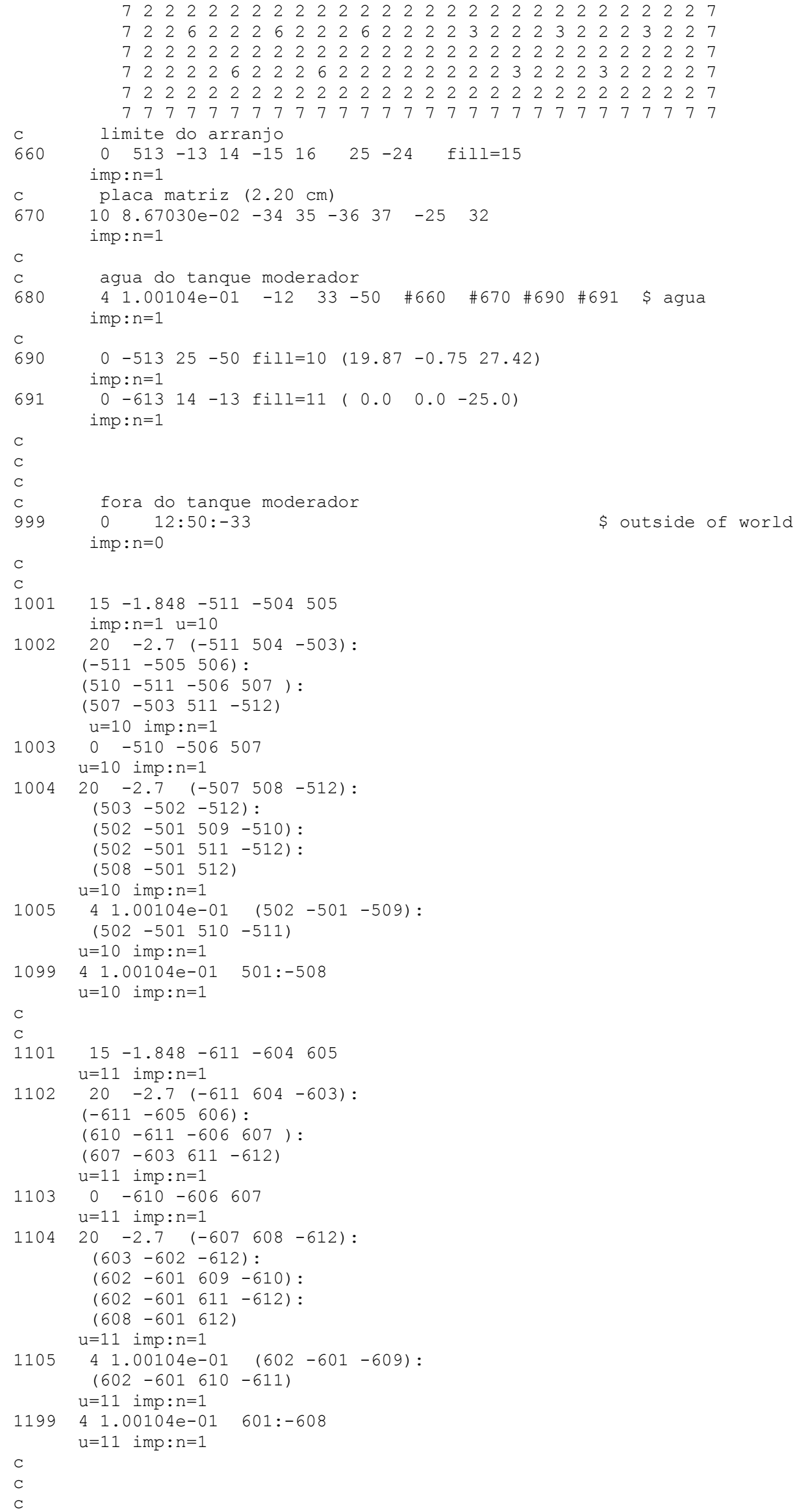




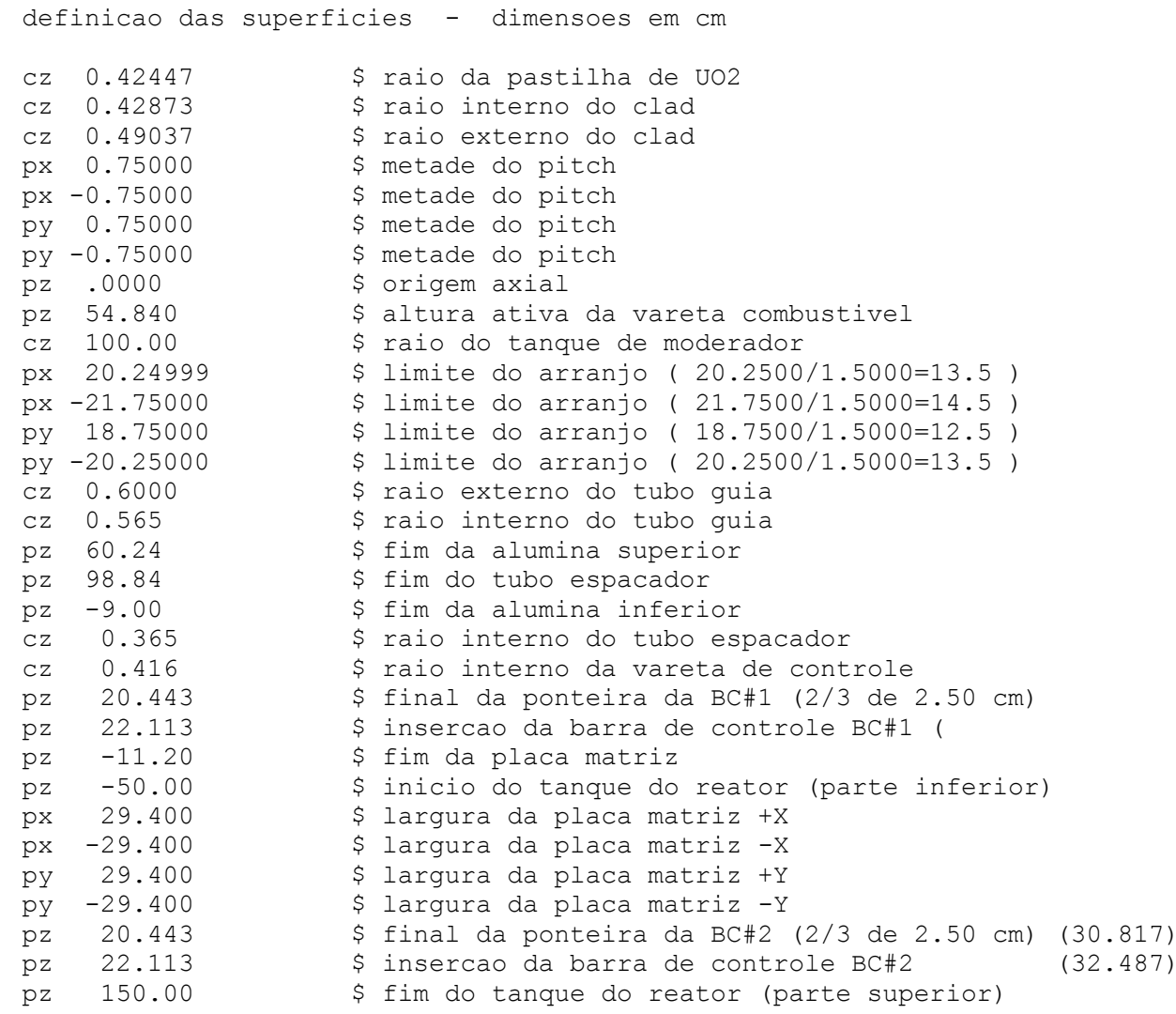


$92235.70 \mathrm{c} 9.9910 \mathrm{e}-04$ $92234.70 \mathrm{c} 9.4528 \mathrm{e}-06$ $12024.70 \mathrm{c} 3.3858 \mathrm{e}-06$ $26054.70 \mathrm{c} 2.3549 \mathrm{e}-07$ $26058.70 \mathrm{c} 1.1369 \mathrm{e}-08$ $42092.70 \mathrm{c} 1.8958 \mathrm{e}-08$ $42096.70 \mathrm{c} 2.1309 \mathrm{e}-08$ $42100.70 \mathrm{c} 1.2302 \mathrm{e}-08$ vazio (gap) $8016.70 \mathrm{c} \quad 0.0001$ encamisamento da vareta combustivel - SS304 - (clad) $26054.70 c 3.5737 e-0326056.70 c 5.4049 e-02$ 26057.70c $1.2269 e-03$ $26058.70 \mathrm{c} 1.5926 \mathrm{e}-04$ $24050.70 \mathrm{c} 7.6190 \mathrm{e}-04 \quad 24052.70 \mathrm{c} 1.4112 \mathrm{e}-02 \quad 24053.70 \mathrm{c} 1.5698 \mathrm{e}-03$ $24054.70 \mathrm{c} 3.8276 \mathrm{e}-04$ $28058.70 \mathrm{c} 5.6294 \mathrm{e}-0328060.70 \mathrm{c} 2.0959 \mathrm{e}-0328061.70 \mathrm{c} 8.9630 \mathrm{e}-05$ $28062.70 \mathrm{c} 2.8081 \mathrm{e}-04 \quad 28064.70 \mathrm{c} \quad 6.9692 \mathrm{e}-05$

$14028.70 \mathrm{c} 6.2899 \mathrm{e}-04 \quad 14029.70 \mathrm{c} \quad 3.0750 \mathrm{e}-05 \quad 14030.70 \mathrm{c} \quad 1.9732 \mathrm{e}-05$ $16032.70 c 1.4885 e-0516033.70 c \quad 1.1393 e-07 \quad 16034.70 c \quad 6.2075 e-07$ $16036.70 \mathrm{c} 2.7850 e-09$

42092.70c 1.3093e-05 42094.70c 7.9875e-06 42095.70c $1.3602 e-05$ $42096.70 \mathrm{c} 1.4103 \mathrm{e}-05$ 42097.70c $7.9912 \mathrm{e}-06$ 42098.70c $1.9985 \mathrm{e}-05$ $42100.70 \mathrm{c} 7.8161 \mathrm{e}-06$

$25055.70 c 1.4645 e-03 \quad 15031.70 c \quad 4.0040 e-05 \quad 6000.70 c 1.1239 e-04$

$27059.70 \mathrm{c} 1.7402 \mathrm{e}-04$

agua

$1001.70 c \quad 6.6736 e-02 \quad 8016.70 c \quad 3.3355 e-02 \quad 8017.70 c \quad 1.2680 e-05$

lwtr.01t

alumina - al2o3

$13027.70 \mathrm{c} 4.4744 \mathrm{e}-02 \quad 8016.70 \mathrm{c} \quad 6.7091 \mathrm{e}-02 \quad 8017.70 \mathrm{c} 2.5504 \mathrm{e}-05$

barra de controle - AG-IN-CD

$47107.70 \mathrm{c} 2.31847 \mathrm{e}-02 \quad 47109.70 \mathrm{c} 2.11443 \mathrm{e}-02$

$49113.70 c \quad 3.3606 e-04 \quad 49115.70 c \quad 7.5157 e-03$

$\begin{array}{llllll}48106.70 c & 3.1590 e-05 & 48108.70 c & 2.2787 e-05 & 48110.70 c & 3.3208 e-04\end{array}$

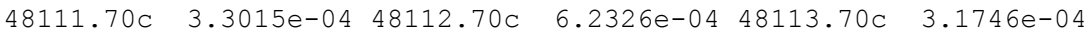

$48114.70 \mathrm{c} \quad 7.4730 \mathrm{e}-04 \quad 48116.70 \mathrm{c} \quad 1.9628 \mathrm{e}-04$

$16032.70 \mathrm{c} \quad 1.7839 \mathrm{e}-04 \quad 16033.70 \mathrm{c} \quad 1.4271 \mathrm{e}-06 \quad 16034.70 \mathrm{c} \quad 7.9243 \mathrm{e}-06$

$16036.70 \mathrm{c} \quad 2.2629 e-08$

$6000.70 \mathrm{c} \quad 1.5052 \mathrm{e}-03 \quad 8016.70 \mathrm{c} \quad 1.7696 \mathrm{e}-03 \quad 8017.70 \mathrm{c} \quad 6.7271 \mathrm{e}-07$

tubo espacador - SS

$26054.70 c \quad 3.7487 e-0326056.70 c 5.6697 e-02 \quad 26057.70 c \quad 1.2870 e-03$

$26058.70 \mathrm{c} 1.6706 \mathrm{e}-04$

$24050.70 \mathrm{c} \quad 7.5984 \mathrm{e}-04 \quad 24052.70 \mathrm{c} \quad 1.4074 \mathrm{e}-02 \quad 24053.70 \mathrm{c} \quad 1.5656 \mathrm{e}-03$

$24054.70 \mathrm{c} 3.8172 \mathrm{e}-04$

$28058.70 \mathrm{c} 4.5313 \mathrm{e}-0328060.70 \mathrm{c} 1.6871 \mathrm{e}-0328061.70 \mathrm{c} 7.2146 \mathrm{e}-05$

$28062.70 c 2.2603 e-0428064.70 c 5.6097 e-05$

$25055.70 \mathrm{c} 1.1581 \mathrm{e}-03$

$14028.70 c 1.0329 e-0314029.70 c 5.0494 e-0514030.70 c \quad 3.2403 e-05$

$15031.70 \mathrm{c} 3.1124 \mathrm{e}-05 \quad 6000.70 \mathrm{c} 2.4078 \mathrm{e}-04 \quad 27059.70 \mathrm{c} \quad 1.1450 \mathrm{e}-04$ tubo guia - SS

$26054.70 \mathrm{c} 3.4489 \mathrm{e}-03 \quad 26056.70 \mathrm{c} 5.2163 \mathrm{e}-02 \quad 26057.70 \mathrm{c} 1.1841 \mathrm{e}-03$

$26058.70 \mathrm{c} 1.5370 \mathrm{e}-04$

$24050.70 c 7.3807 e-04 \quad 24052.70 c 1.3671 e-02 \quad 24053.70 c 1.5207 e-03$

$24054.70 \mathrm{c} 3.7078 \mathrm{e}-04$

$28058.70 \mathrm{c} 6.2785 \mathrm{e}-03 \quad 28060.70 \mathrm{c} 2.3376 \mathrm{e}-03 \quad 28061.70 \mathrm{c} 9.9965 \mathrm{e}-05$

$28062.70 \mathrm{c} 3.1318 \mathrm{e}-04 \quad 28064.70 \mathrm{c} 7.7727 \mathrm{e}-05$

$25055.70 \mathrm{c} 1.1501 \mathrm{e}-03$

$14028.70 \mathrm{c} 6.1266 \mathrm{e}-04 \quad 14029.70 \mathrm{c} 2.9952 \mathrm{e}-0514030.70 \mathrm{c} 1.9221 \mathrm{e}-05$

$15031.70 \mathrm{c} 4.5000 \mathrm{e}-05 \quad 6000.70 \mathrm{c} 8.8968 \mathrm{e}-05$ placa matriz - SS

$26054.70 \mathrm{c} 3.63630 \mathrm{e}-0326056.70 \mathrm{c} 5.49964 \mathrm{e}-02 \quad 26057.70 \mathrm{c} 1.24843 \mathrm{e}-03$ $26058.70 \mathrm{c} 1.62047 \mathrm{e}-04$

$24050.70 \mathrm{c} 7.56501 \mathrm{e}-0424052.70 \mathrm{c} 1.40122 \mathrm{e}-0224053.70 \mathrm{c} 1.55868 \mathrm{e}-03$ $24054.70 c \quad 3.80042 e-04$

$28058.70 \mathrm{c} 5.28566 \mathrm{e}-0328060.70 \mathrm{c} 1.96791 \mathrm{e}-0328061.70 \mathrm{c} 8.41568 \mathrm{e}-05$ $28062.70 \mathrm{c} 2.63658 \mathrm{e}-04 \quad 28064.70 \mathrm{c} 6.54358 \mathrm{e}-05$

$14028.70 \mathrm{c} \quad 8.01962 \mathrm{e}-04 \quad 14029.70 \mathrm{c} 3.92060 \mathrm{e}-05 \quad 14030.70 \mathrm{c} 2.51595 \mathrm{e}-05$ $16032.70 \mathrm{c} \quad 4.25282 \mathrm{e}-06 \quad 16033.70 \mathrm{c} \quad 3.25504 \mathrm{e}-08 \quad 16034.70 \mathrm{c} \quad 1.77356 \mathrm{e}-07$ $16036.70 \mathrm{c} 7.95715 \mathrm{e}-10$

$42092.70 \mathrm{c} 4.62114 \mathrm{e}-06 \quad 42094.70 \mathrm{c} 2.81913 \mathrm{e}-06 \quad 42095.70 \mathrm{c} \quad 4.80079 \mathrm{e}-06$ $42096.70 \mathrm{c} 4.97759 \mathrm{e}-06 \quad 42097.70 \mathrm{c} 2.82043 \mathrm{e}-06 \quad 42098.70 \mathrm{c} 7.05364 \mathrm{e}-06$ $42100.70 \mathrm{c} 2.75861 \mathrm{e}-06$

$25055.70 \mathrm{c} 1.25030 \mathrm{e}-03$ 15031.70c $5.54400 \mathrm{e}-05$ 6000.70c $7.94260 \mathrm{e}-05$ encamisamento das barras de controle e de seguranca - SS304 $26054.70 \mathrm{c} 3.50278 \mathrm{e}-0326056.70 \mathrm{c} 5.29770 \mathrm{e}-02$ 26057.70c $1.20259 \mathrm{e}-03$ 


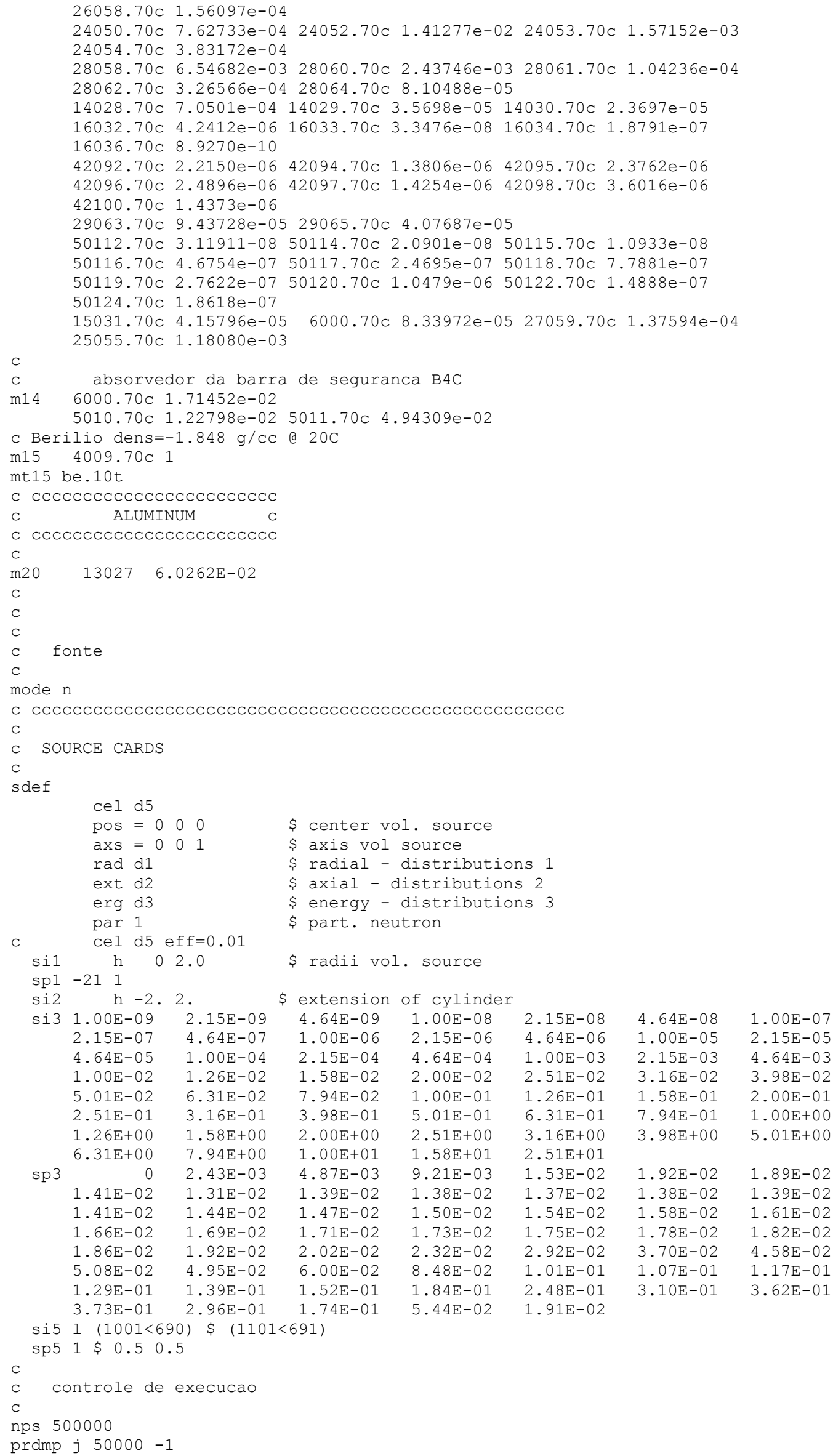




\section{F.3. Entrada de dados para TORT}

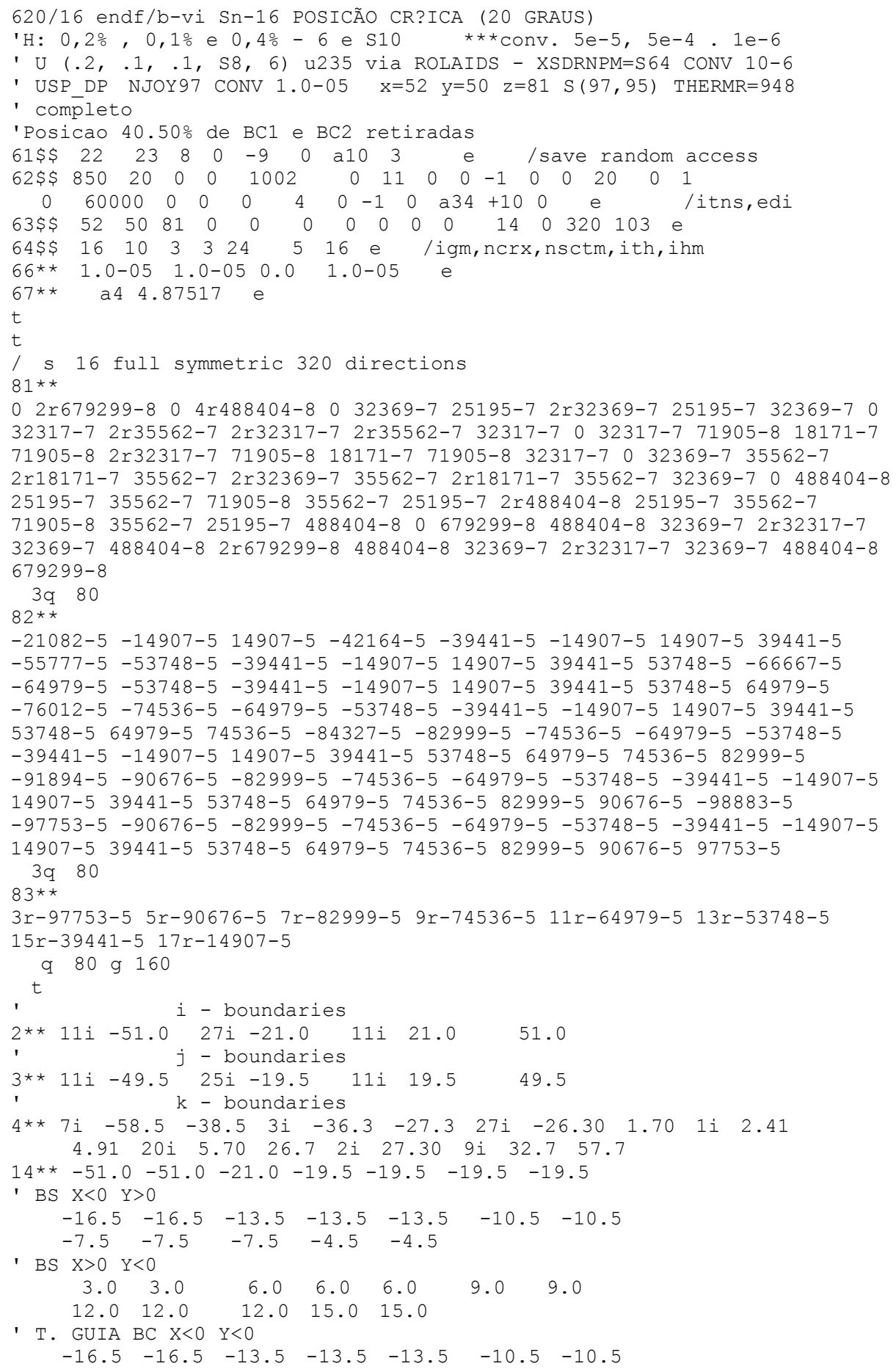




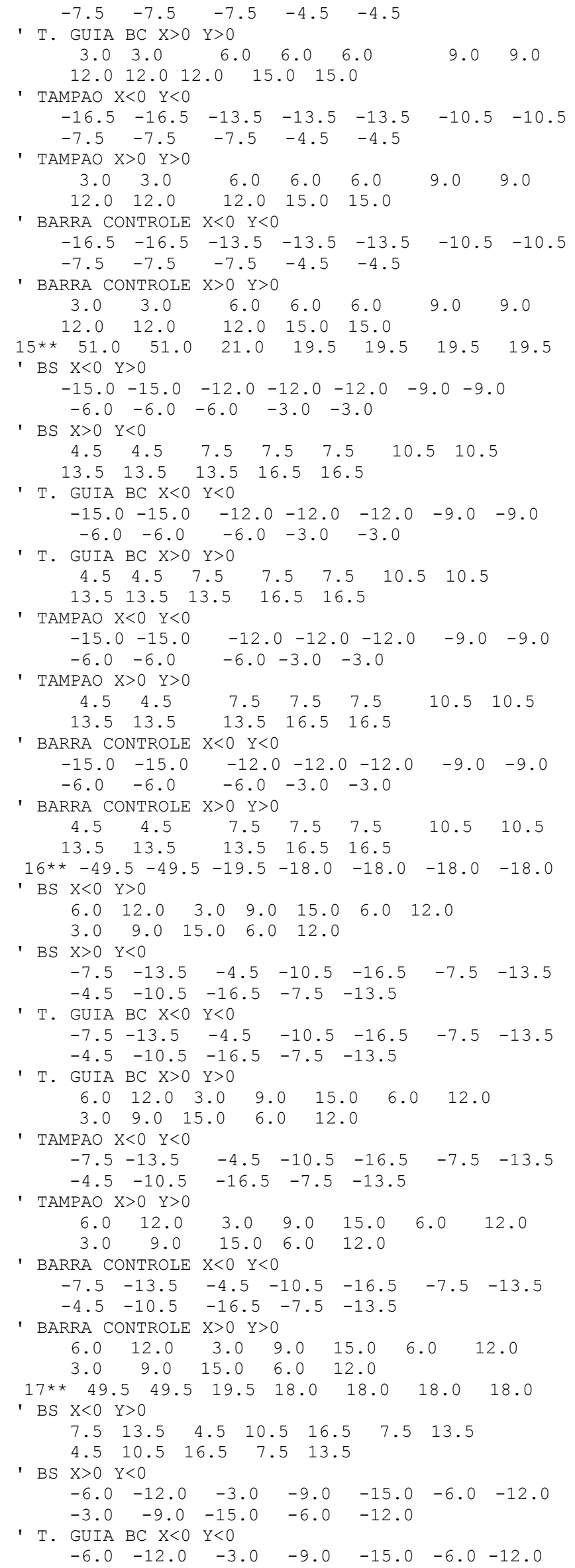


$\begin{array}{lllll}-3.0 & -9.0 & -15.0 & -6.0 & -12.0\end{array}$

' T. GUIA BC X>0 Y>0

$\begin{array}{lllllll}7.5 & 13.5 & 4.5 & 10.5 & 16.5 & 7.5 & 13.5\end{array}$

$\begin{array}{lllll}4.5 & 10.5 & 16.5 & 7.5 & 13.5\end{array}$

- TAMPAO $\mathrm{X}<0 \quad \mathrm{Y}<0$

$\begin{array}{lllllll}-6.0 & -12.0 & -3.0 & -9.0 & -15.0 & -6.0 & -12.0\end{array}$

$\begin{array}{lllll}-3.0 & -9.0 & -15.0 & -6.0 & -12.0\end{array}$

- TAMPAO $X>0 \quad Y>0$

$\begin{array}{lllllll}7.5 & 13.5 & 4.5 & 10.5 & 16.5 & 7.5 & 13.5\end{array}$

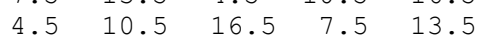

' BARRA CONTROLE $\mathrm{X}<0 \quad \mathrm{Y}<0$

$\begin{array}{lllllll}-6.0 & -12.0 & -3.0 & -9.0 & -15.0 & -6.0 & -12.0\end{array}$

$-3.0-9.0 \quad-15.0 \quad-6.0-12.0$

- BARRA CONTROLE X>0 $\mathrm{Y}>0$

$\begin{array}{lllllll}7.5 & 13.5 & 4.5 & 10.5 & 16.5 & 7.5 & 13.5\end{array}$

$\begin{array}{lllll}4.5 & 10.5 & 16.5 & 7.5 & 13.5\end{array}$

$18 * * \quad-58.5 \quad-38.5 \quad-38.5 \quad-36.3 \quad-27.3 \quad 27.3 \quad 32.7$

' $B S \quad X<0 \quad Y>0$

$\begin{array}{llllllll}-38.5 & -38.5 & -38.5 & -38.5 & -38.5 & -38.5 & -38.5\end{array}$

$\begin{array}{lllll}-38.5 & -38.5 & -38.5 & -38.5 & -38.5\end{array}$

' $B S \quad X>0 \quad Y<0$

$\begin{array}{lllllll}-38.5 & -38.5 & -38.5 & -38.5 & -38.5 & -38.5 & -38.5\end{array}$

$\begin{array}{lllll}-38.5 & -38.5 & -38.5 & -38.5 & -38.5\end{array}$

' T. GUIA BC $X<0 \quad Y<0$

$\begin{array}{llllllll}-38.5 & -38.5 & -38.5 & -38.5 & -38.5 & -38.5 & -38.5\end{array}$

$\begin{array}{lllll}-38.5 & -38.5 & -38.5 & -38.5 & -38.5\end{array}$

' T. GUIA BC $\mathrm{X}>0$ Y $>0$

$\begin{array}{llllllll}-38.5 & -38.5 & -38.5 & -38.5 & -38.5 & -38.5 & -38.5\end{array}$

$-38.5-38.5-38.5-38.5-38.5$

- TAMPAO $\mathrm{X}<0 \quad \mathrm{Y}<0$

$\begin{array}{lllll}-6.86 & -6.86 & -6.86 & -6.86 & -6.86\end{array}$

$\begin{array}{lllll}-6.86 & -6.86 & -6.86 & -6.86 & -6.86\end{array}$

$-6.86-6.86$

1 TAMPAO $\mathrm{X}>0 \quad \mathrm{Y}>0$

$\begin{array}{lllll}-6.86 & -6.86 & -6.86 & -6.86 & -6.86\end{array}$

$\begin{array}{lllll}-6.86 & -6.86 & -6.86 & -6.86 & -6.86\end{array}$

$-6.86-6.86$

' BARRA CONTROLE $\mathrm{X}<0 \quad \mathrm{Y}<0$

$\begin{array}{lllll}-5.19 & -5.19 & -5.19 & -5.19 & -5.19\end{array}$

$\begin{array}{lllll}-5.19 & -5.19 & -5.19 & -5.19 & -5.19\end{array}$

$-5.19-5.19$

1 BARRA CONTROLE X>0 Y>0

$\begin{array}{lllll}-5.19 & -5.19 & -5.19 & -5.19 & -5.19\end{array}$

$\begin{array}{lllll}-5.19 & -5.19 & -5.19 & -5.19 & -5.19\end{array}$

$-5.19-5.19$

$\begin{array}{llllllll}19 * * & -38.5 & 57.7 & -36.3 & -27.3 & 27.3 & 32.7 & 57.7\end{array}$

' BS $\mathrm{X}<0 \quad \mathrm{Y}>0$

$\begin{array}{lllllll}57.7 & 57.7 & 57.7 & 57.7 & 57.7 & 57.7 & 57.7\end{array}$

$\begin{array}{lllll}57.7 & 57.7 & 57.7 & 57.7 & 57.7\end{array}$

, BS $\mathrm{X}>0 \quad \mathrm{Y}<0$

$\begin{array}{lllllll}57.7 & 57.7 & 57.7 & 57.7 & 57.7 & 57.7 & 57.7\end{array}$

$\begin{array}{lllll}57.7 & 57.7 & 57.7 & 57.7 & 57.7\end{array}$

' T. GUIA BC $X<0 \quad Y<0$

$\begin{array}{lllll}-6.86 & -6.86 & -6.86 & -6.86 & -6.86\end{array}$

$\begin{array}{lllll}-6.86 & -6.86 & -6.86 & -6.86 & -6.86\end{array}$

$-6.86-6.86$

' T. GUIA BC $\mathrm{X}>0 \quad \mathrm{Y}>0$

$\begin{array}{lllll}-6.86 & -6.86 & -6.86 & -6.86 & -6.86\end{array}$

$\begin{array}{lllll}-6.86 & -6.86 & -6.86 & -6.86 & -6.86\end{array}$

$-6.86-6.86$

, TAMPAO $\mathrm{X}<0 \quad \mathrm{Y}<0$

$\begin{array}{lllll}-5.19 & -5.19 & -5.19 & -5.19 & -5.19\end{array}$

$\begin{array}{lllll}-5.19 & -5.19 & -5.19 & -5.19 & -5.19\end{array}$

$-5.19-5.19$

' TAMPAO $\mathrm{X}>0 \quad \mathrm{Y}>0$

$\begin{array}{lllll}-5.19 & -5.19 & -5.19 & -5.19 & -5.19\end{array}$

$\begin{array}{lllll}-5.19 & -5.19 & -5.19 & -5.19 & -5.19\end{array}$

$-5.19-5.19$

' BARRA CONTROLE $X<0 \quad \mathrm{Y}<0$

$\begin{array}{lllllll}57.7 & 57.7 & 57.7 & 57.7 & 57.7 & 57.7 & 57.7\end{array}$

$\begin{array}{lllll}57.7 & 57.7 & 57.7 & 57.7 & 57.7\end{array}$

' BARRA CONTROLE X>0 $Y>0$

$\begin{array}{lllllll}57.7 & 57.7 & 57.7 & 57.7 & 57.7 & 57.7 & 57.7\end{array}$

$\begin{array}{lllll}57.7 & 57.7 & 57.7 & 57.7 & 57.7\end{array}$

- ESPECTRO DE FISSAO ENDF/B-VII.O 


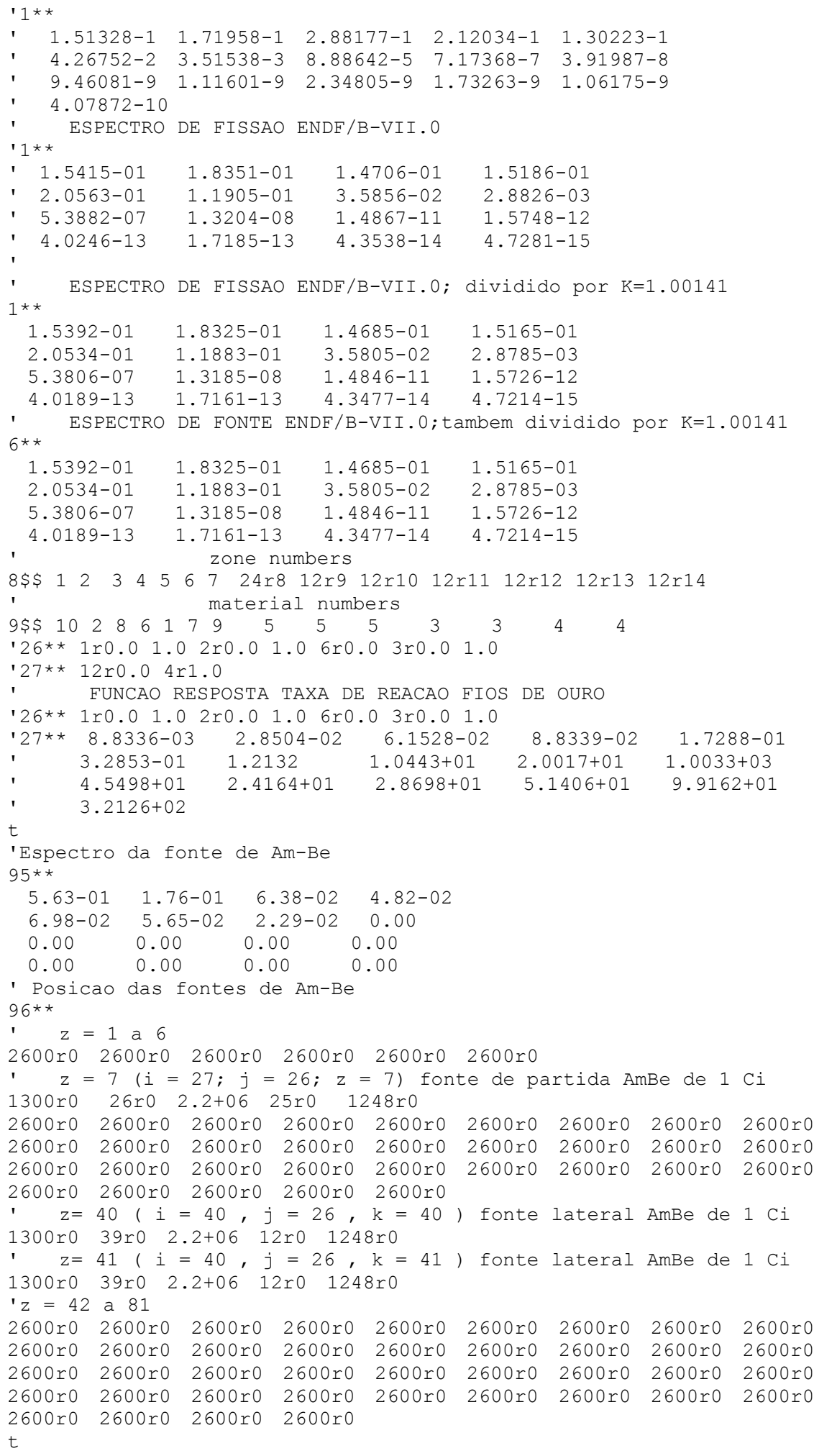




\section{REFERÊNCIAS BIBLIOGRÁFICAS}

Agilent Technologies, 2000, The Fundamentals of Signal Analysis; Application Notes 243, http://cp.literature.agilent.com/litweb/pdf/5952-8898E.pdf .

Baeten, P., and Abderrahim, H., 2003 "Reactivity monitoring in ADS, application to the myrrha ADS project," Prog. nucl. Energy, 43, 1-4, p. 413.

Bell, G. I., Glasstone, S., 1979. Nuclear Reactor Theory. Van Nostrand Reinhold, New York.

Bendat, J. S., Piersol, A. G., 2000. Random Data: Analysis and Measurement Procedures. John Wiley \& Sons. Third Edition.

Briggs, J.B., editor, 2012. International Handbook of evaluated Criticality Safety Benchmark Experiments NEA/NSC/ DOC(95)03/I, Nuclear Energy Agency, Paris (September 2012 Edition).

Brown, F., B., 2005, Fundamentals of Monte Carlo Particle Transport, Los Alamos National Laboratory report LA-UR-05-4983 (2005). 143.

Carlson, B.G.;Bell, G. I., 1958, Solution of the Transport Equation by the $\mathrm{Sn}$ Method, Proc.U. N. Intern. Conf. Peaceful Uses At. Energy, 2nd, Geneva P/2386.

Carlson, B.G., Lathrop, K.D., 1965, Transport Theory - The Method of Discrete Orndinates. LA-3251-MS.

Carluccio, Th., 2011, Implementação e qualificação de metodologia de cálculos neutrônicos em reatores subcríticos acionados por fonte externa de nêutrons e aplicações, Tese de doutorado, IPEN.

Carta, M., et al., 2004, Reactivity Assessment and Spatial Time-effects from the MUSE Kinetics Experiment, in PHYSOR 2004, Chicago, Illinois, April 25-29, CD Room, American Nuclear Society.

Chadwick, M.B. et al., 2006. ENDF/B-VII.0 next generation evaluated nuclear data library for nuclear science and technology. Nucl. Data Sheets 107, 2931-3060.

Cohn, C.E., 1959, Determination of Reactor Kinetics Parameters by Pile Noise Analysis, Nucl. Sci. Eng., 5, 331.

Cohn, C.E., 1960, Simplified Theory of Pile Noise, Nucl. Sci. Eng., 7, 472.

Cohn, C.E., 1962, Reflected-Reactors Kinetics, Nucl. Sci. Eng. 13, 12-17. 
Diniz, R., 2005, Obtenção das Constantes de Decaimento e Abundâncias Relativas de Nêutrons Atrasados Através da Análise de Ruído em Reatores de Potência Zero, Tese de doutorado, IPEN.

Diniz, R., Dos Santos, A., 2006, Experimental Determination of the Decay Constants and Abundances of Delayed Neutrons by Means of Reactor Noise Analysis, Nucl. Sci. Eng., 152, 2, p. 125.

Dos Santos, A., Abe, A. Y., Mendonça, A. G., Fanaro, L. C. C. B., Andrade e Silva , G. S., 2000. Criticality Analyses Based on the Coupled NJOY/AMPX-II/TORT Systems. PHYSOR-2000. Pittsburgh, Pennsylvania.

Dos Santos, A., et al., 2004a. LEU-COMP-THERM-077: Critical Loading Configurations of the IPEN/MB-01 Reactor. In: Briggs, J. Blair (Ed.), International Handbook of Evaluated Criticality Safety Benchmark Experiments. NEA/NSC/DOC (95)03/I, Paris, September.

Dos Santos, A., et al, 2004b, The Experimental Determination of the Relative Abundances and Decay Constants of Delayed Neutrons of the IPEN/MB-01 Reactor, PHYSOR 2004, Chicago, Illinois, April 25-29.

Dos Santos, A., et al., 2005. The Inversion Point of the Isothermal Reactivity Coefficient of the IPEN/MB-01 Reactor-II: Theoretical Analysis, Nucl. Sci. and Eng. 151, 237-250.

Dos Santos, A., et al., 2006a, "A proposal of benchmark for $\beta_{\text {eff }}, \beta_{\text {eff }} / \Lambda$ and $\Lambda$ of thermal reactors fueled with slight enriched uranium", Ann. Nucl. Energy, 33, p. 848.

Dos Santos, A., et al., 2006b. LEU-COMP-THERM-082: Critical Loading Configurations of the IPEN/MB-01 Considering Low Enriched Fuel and Burnable Poison Rods. International Handbook of Evaluated Criticality Safety Benchmark Experiments. NEA/NSC/DOC (95)03/I, Paris, September.

Dos Santos, A., et al., 2008. LEU-COMP-THERM-043: Critical Loading Configurations of the IPEN/MB-01 Reactor with a Heavy SS-304 Reflector. International Handbook of Evaluated Criticality Safety Benchmark Experiments. NEA/NSC/DOC (95)03/I, Paris, September.

Dos Santos, A., et al., 2009. IPEN (MB01)-LWR-COEF-KIN-RESR-001: Reactor Physics experiments in the IPEN/MB-01 Research Reactor Facility. International Handbook of Evaluated Reactor Physics Benchmark Experiments. Paris: NEA/NSC, pp. 1-142.

Dulla, S., et al., 2006. Kinetic Parameters for Source Driven Systems. PHYSOR-2006. 
ANS Topical Meeting on Reactor Physics Organized and hosted by the Canadian Nuclear Society. Vancouver, BC, Canada, September.

Gandini, A., 2001. HGPT Based Sensitivity Time-Dependent Method for the Analysis of Subcritical Systems. Annals of Nuclear Energy. Vol. 28, pp. 1193-1217.

Gandini, A., 2004. ADS subcriticality evaluation based on the generalized reactivity concept", Annals of Nuclear Energy. Vol. 31, pp. 813-821

Gandini, A., Salvatores, M., 2002. The Physics of Subcritical Multiplying Systems. Journal of Nuclear Science and Technology. Vol. 39, No. 6, pp. 673-686.

Gonzales, R., et al., 2004, The MUSE4 Pulsed Neutron Source Experiment, in PHYSOR 2004, Chicago, Illinois, April 25-29, CD Room, American Nuclear Society.

Greene, N. M., et all, 1976. AMPX-II A Modular Code System for Generation Coupled Multigroup Neutron-Gamma Libraries from ENDF/B. ORNL-TM-3706.

Hetrick, D. L., 1971. Dynamics of Nuclear Reactors. The University of Chicago Press.

Hoogenboom, J.E., Van Der Sluijs, A.R., 1988. Neutron source strength Determination for on-line reactivity measurements. Annals of Nuclear Energy. Vol. 15, Issue 12, pp.553-559.

Keepin, G.R., 1965, Physics of Nuclear Kinetics, Addison-Wesley.

Kiedrowski B., Brown, F., Wilson, P., 2009, Calculating kinetic parameters and reactivity changes with continuous-energy Monte Carlo.

Kitamura, Y., Matoba, M., Misawa, T., Unesaki, H., Shiroya, S., 1999. Reactor Noise Experiments by Using Acquisition System for Time Series Data of Pulse Train. Journal of Nuclear Science and Technology, Vol. 36, No.8, pp. 653-660.

Knoll, Glenn F. (editor) 2000, Radiation Detection and Measurament. Jonh Wiley Sons Inc.

Kuramoto, R., Dos Santos, A., 2003, Determinação Experimental dos Parâmetros Cinéticos $\beta_{\text {eff }}, \Lambda$ e $\beta_{\text {eff }} \Lambda$ do Reator IPEN/MB-01, Projeto de doutoramento FAPESP n. 03/01261-0.

Kuramoto, R., Dos Santos, A.,., 2006, "Absolute Measurement of $\beta_{\text {eff }}$ based on Rossi$\alpha$ experiments and the Two-Region Model in the IPEN/MB-01 Research Reactor," Nucl. Sci. Eng. .

Kuramoto, R., 2007, Desenvolvimento de uma Metodologia Baseada no Modelo de 
Duas-Regiões e em Técnicas de Análise de Ruío Microscópico para a Medida Absoluta dos Parâmetros Cinéticos $\beta_{\text {eff, }}, \Lambda$ e $\beta_{\text {eff }} / \Lambda$ do Reator IPEN/MB-01. Tese de doutorado IPEN.

Maiorino, J. R.,et al., 2003, The Utilization of Accelerators in Sub Critical Systems for Energy Generation and Nuclear Waste Transmutation-The World Status and a Proposal of a National R\&D Program, Brazilian Journal of Physics, 33, 2.

Maiorino, J. R., 2005, Accelerator Driven System: An Innovative Reactor to be Used as Dedicated Waste Burner and a Multipurpose Neutron Source: The Status of the Art, International Nuclear Atlantic Conference-INAC 2005, Santos-SP, Brazil, August 28 to September 2,

MacFarlane, R. E., Muir, D.W., Bouicort ,R. M., 1994. NJOY - Code System for Producing Pointwise and Multigroup Neutron and Photon Cross Sections from ENDF Data. LA-12740-M, Los Alamos National Laboratory, Los Alamos, NM.

MCNP-5 X-5 Monte Carlo Team, 2003. MCNP - A General Monte Carlo N-Particle Transport Code, Version 5, LA-UR-03-1987, April.

Mellier, F.(coordinator), 2005, The MUSE experiment for subcritical neutronics validation, Deliverable no 8 - Final report.

Moore, M.N., 1958, The Determination of Reactor Transfer Funcions from Measurements at Steady Operation, Nucl. Sci. Eng..

Moore, M.N., 1959, The Power Noise Transfer Function of a Reactor, Nucl. Sci. Eng., 6,448 .

Naing, W., Tsuji, M., Shimazu, Y., 2004, Subcriticality Measurement of Pressurized Water Reactors during Criticality Approach using a Digital Reactivity Meter, J. Nucl. Sci. Technol., 42[2], 145-152.

Nakamura, S., 1986, Computational Methods in Engineering and Science, R. E. Krieger Publishing Company, Malabar, FL.

NEA Data bank, 2006. JEFF Report 21: the JEFF-3.1 Nuclear Data Library, website : http://www.nea.fr/html/dbdata/nds jefreports/jeffreport-21/index.html

Oblozinsky, O., Herman M., 2006. Special Issue on Evaluated Nuclear Data File ENDF/B-VII.0, Nuclear Data Sheets, 107, No. 12.

Persson. C.M., et al., 2005, Analysis of reactivity determination methods in the subcritical experiment YALINA, Nuclear Instruments and Methods in Physics Research A 554, 374-383. 
Persson, C.M., 2007, Reactivity Assessment in Subcritical Systems, KTH Engineering Sciences, Licentiate Thesis in Physics, Stockholm, Sweden.

Pratibha, A., et al., 2006, Accelerator driven systems (ADS) for energy production and waste transmutation: International trends in R\&D, Prog. nucl. Energy, 48, 2, p. 91.

Rhoades, W.A., 1975. The GIP Program for Preparation of Group-Organized Cross Section Libraries. PSR-75, ORNL.

Rhoades, W.A., Childs, R.L., 1987, The TORT Three-Dimensional Discrete Ordinates Neutrons/Photon Transport Code, Oak Ridge National Laboratory, Oak Ridge, TN, ORNL-6268.

Rhoades, W.A., Simpson, D. B., 1991. The TORT Three-Dimensional Discrete Ordinates Neutron/Photon Transport Code. ORNL/TM-13221.

Rubbia, C., et al, 2004.The TRADE Experiment: Status of the Project and Physics of the Spallation Target. PHYSOR-2004, The Physics of Fuel Cycles and Advanced Nuclear Systems: Global Developments, Chicago, Illinois, April. On CDROM, American Nuclear Society, Lagrange Park, IL.

Salvatores, M., et al., 1996. MUSE-1: A first experiment at MASURCA to validate the physics of subcritical multiplying systems relevant to ADS. $2^{\text {nd }}$ ADTT Conf. Kalmar, Sweden, June.

Shi, Y.Q., Zhu, Q.F., Tao, H., 2005. Review and research of the neutron source multiplication method in nuclear critical safety. Nuclear Technology. Vol. 149, No. 1, pp. 122-127.

Shibata, K. et al., Japanese Evaluated Nuclear Data Library Version 3 Revision-3: JENDL 3.3., J. Nucl. Sci. Technol. 39, 1125, 2002.

Shimazu, Y., Naing, W., 2005. Some Technical Issues on Continuous Subcriticality Monitoring by a Digital Reactivity Meter During Criticality Approach. Journal of Nuclear Science and Technology. Vol. 42, No. 6, pp. 515-524

Simmons, B.E., King, J.S., 1958, A Pulsed Technique for Reactivity Determination, Nucl. Sci. Eng, 3, pp. 595-608.

Sjöstrand, N.G., 1956, Measurement on a subcritical reactor using a pulsed neutron source, Arkiv för fysik 11, 13.

Spriggs, G.D., Busch, R.D., Williams, J.G., 1997, Two-region kinetic model for reflected reactor. Ann. Nucl. Energy, v.24, n 3, p. 205-250. 
Suzuki, E., 1966. A Method for Measuring Absolute Reactor Power Through Neutron Fluctuation. Journal of Nuclear Science and Technology. Vol. 3, No. 3, pp. 98-105.

Uhrig., R. E., 1970, Random noise techniques in nuclear reactor system, The Ronald Press company 